

\section{Propositions}

1. The receptor-like kinases BIR2 and BIR3 negatively regulate complex formation of RLP receptors with their regulatory kinases SOBIR1 and BAK1 to control plant defence.

(this thesis)

2. The receptor-like protein CLV2 regulates defence responses mediated by cell surface receptors through controlling the accumulation of SOBIR1 and BAK1 proteins.

(this thesis)

3. Considering that human SelO is a pseudokinase that transfers AMP to its substrates (Sreelatha et al., 2018 Cell), such kinases should be termed atypical kinases rather than pseudokinases.

4. To exclude the presence of potential off-target mutations, mutants generated by CRISPR-Cas-based techniques must be subjected to whole genome sequencing.

5. Comprehensive application of biometrics systems will make our future life more convenient in all aspects, including travelling and health care.

6. Organic food can only be promoted based on aspects related to lower pesticide exposure and having less environmental impact, but should not be promoted based on its safety and nutritional value.

Propositions belonging to the thesis, entitled

'Regulation and activation of SOBIR1-containing receptor complexes

involved in plant immune signalling'

Jinbin Wu

Wageningen, 3 February 2020 
Regulation and activation of SOBIR1-containing receptor complexes involved in plant immune signalling

Jinbin Wu 


\section{Thesis committee}

\section{Promotor}

Prof. Dr Bart P.H.J. Thomma

Professor of Phytopathology

Wageningen University \& Research

\section{Co-promotor}

Dr Matthieu H.A.J. Joosten

Associate professor, Laboratory of Phytopathology

Wageningen University \& Research

\section{Other members}

Prof. Dr Dolf Weijers, Wageningen University \& Research

Prof. Dr Thorsten Nürnberger, University of Tübingen, Germany

Dr Aska Goverse, Wageningen University \& Research

Prof. Dr Guido F.J.M. van den Ackerveken, Utrecht University

This research was conducted under the auspices of the Graduate School Experimental Plant Sciences (EPS). 


\title{
Regulation and activation of SOBIR1-containing receptor complexes involved in plant immune signalling
}

\author{
Jinbin Wu
}

\section{Thesis}

submitted in fulfilment of the requirements for the degree of doctor at Wageningen University

by the authority of the Rector Magnificus,

Prof. Dr A.P.J. Mol,

in the presence of the

Thesis Committee appointed by the Academic Board

to be defended in public

on Monday February 32020

at 4 p.m. in the Aula. 
Jinbin Wu

Regulation and activation of SOBIR1-containing receptor complexes involved in plant immune signalling,

184 pages.

$\mathrm{PhD}$ thesis, Wageningen University, Wageningen, the Netherlands (2020)

With references, with summary in English.

ISBN: 978-94-6395-182-1

DOI: $10.18174 / 504639$ 


\section{Table of contents}

\section{Chapter 1}

General introduction

\section{Chapter 2}

Receptor-like kinases BIR2 and BIR3 interact with SOBIR1 to negatively regulate Cf-

4 signalling. 33

\section{Chapter 3}

The bacterial effector AvrPto targets the regulatory co-receptor SOBIR1 and suppresses defence signalling mediated by the receptor-like protein Cf-4 55

\section{Chapter 4}

An EFR-Cf-9 chimera confers enhanced resistance to bacterial pathogens by SOBIR1- and BAK1-dependent recognition of elf18

\section{Chapter 5}

Functional analysis of CLV2-containing complexes and identification of components required for the response to the CLV3 peptide in Arabidopsis thaliana 99

\section{Chapter 6}

General discussion

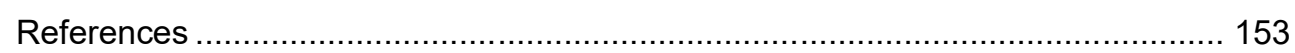

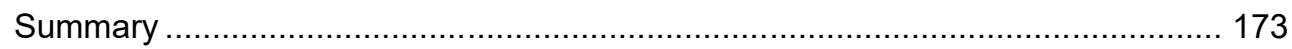

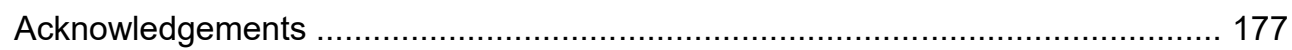

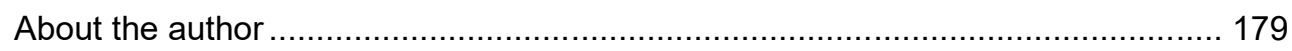

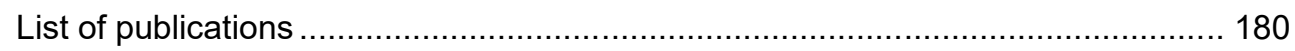

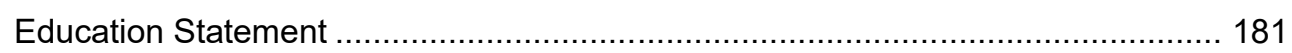



Chapter 1

\section{General introduction}




\section{Introduction}

Plants are constantly attacked by invading microbial pathogens and have developed two layers of defence to protect themselves against invaders (Dodds and Rathjen, 2010; Jones and Dangl, 2006). The first layer is mediated by cell surface receptors, which are previously known as pattern recognition receptors that recognize microbeassociated molecular patterns (MAMPs) to initiate MAMP-triggered immunity (Dodds and Rathjen, 2010; Jones and Dangl, 2006; van der Burgh and Joosten, 2019). Cell surface receptors recognize extracellular immunogenic patterns (ExIPs) to initiate downstream immune responses, eventually leading to extracellularly-triggered immunity (ExTI) (van der Burgh and Joosten, 2019). An ExIP can be any ExTItriggering extracellular molecule, including MAMPs, host-derived extracellular danger signals and microbial extracellular effectors (Boller and Felix, 2009; Cook et al., 2015; Dodds and Rathjen, 2010; Gust et al., 2017; Jones and Dangl, 2006; Kanyuka and Rudd, 2019; Thomma et al., 2011; van der Burgh and Joosten, 2019). Adapted, specialised pathogens suppress ExTI to colonize their host plants with the employment of effectors, of which the ones translocated into host cells can be recognized by intracellular receptors (van der Burgh and Joosten, 2019). Intracellular receptors are mainly nucleotide-binding leucine-rich repeat (NB-LRR) proteins, and activation of these receptors results in intracellularly-triggered immunity $(\mathrm{InTI})$, which constitutes the second layer of plant defence (Dodds and Rathjen, 2010; Jones and Dangl, 2006; van der Burgh and Joosten, 2019).

$\operatorname{InTI}$ is generally associated with programmed cell death, which is known as the hypersensitive response (HR) (Dodds and Rathjen, 2010; Jones and Dangl, 2006; van der Burgh and Joosten, 2019). Activation of cell surface receptors mostly does not trigger the development of an $\mathrm{HR}$, although some cell surface receptors do cause an HR upon recognition of their matching ExIP (Dodds and Rathjen, 2010; Jones and Dangl, 2006; van der Burgh and Joosten, 2019). However, in principle, both ExTI and $\mathrm{InTI}$ are sufficient to provide resistance to pathogens (van der Burgh and Joosten, 2019).

\section{Cell surface receptors in plant immunity}

Cell surface receptors are either receptor-like kinases (RLKs) or receptor-like proteins (RLPs) (Boutrot and Zipfel, 2017; Monaghan and Zipfel, 2012). RLKs contain a ligandbinding ectodomain, a transmembrane domain and an intracellular kinase domain, while RLPs are generally similar to RLKs but lack an intracellular kinase domain (Boutrot and Zipfel, 2017; Monaghan and Zipfel, 2012). Based on the ligand-binding motifs present in their ectodomains, cell surface receptors are further subdivided into LRR-, lysin motif-, lectin- and epidermal growth factor-like domain-containing RLKs 
and RLPs (Boutrot and Zipfel, 2017; Monaghan and Zipfel, 2012). RLKs and RLPs containing variable amounts of LRRs in their ectodomains (LRR-RLKs and LRR-RLPs, further referred to as RLKs and RLPs, respectively) are the two major groups of cell surface receptors that mediate immunity against infection by a range of pathogens in various plant species (Boutrot and Zipfel, 2017; Li et al., 2016c; van der Burgh and Joosten, 2019; Wan et al., 2019).

Arabidopsis thaliana (further referred to as Arabidopsis) FLAGELLIN-SENSING 2 (FLS2) is a well-characterized RLK involved in immunity (Gómez-Gómez and Boller, 2000). FLS2 contains 28 LRRs in its ectodomain that directly binds the typical MAMP flg22, which is an epitope of bacterial flagellin, and mediates immunity against bacterial infection (Felix et al., 1999; Gómez-Gómez and Boller, 2000; Sun et al., 2013b). Arabidopsis ELONGATION FACTOR-TU RECEPTOR (EFR) is another wellcharacterized RLK that contains 21 LRRs in its ectodomain and recognizes the MAMP elf18, which is an epitope of bacterial elongation factor-Tu (EF-Tu), to mediate immunity against bacterial infection (Zipfel et al., 2006). FLS2 is widely expressed in plants, whereas EFR is restricted to the Brassicaceae (Chinchilla et al., 2006; GómezGómez and Boller, 2000; Hann and Rathjen, 2007; Robatzek et al., 2007; Takai et al., 2008; Zipfel et al., 2006).

Tomato (Solanum lycopersicum, Sl) Cf-4 and Cf-9 are two well-studied RLPs, which contain 25 and 27 LRRs in their ectodomains, respectively, and share an identical Cterminal half including the transmembrane domain and the cytoplasmic tail (Jones et al., 1994; Thomas et al., 1997). Cf-4 and Cf-9 confer resistance to strains of the biotrophic fungal pathogen Cladosporium fulvum that secrete the cysteine-rich effectors avirulence factor 4 (Avr4) and Avr9, respectively (Jones et al., 1994; Joosten et al., 1994; Thomas et al., 1997; van den Ackerveken et al., 1992). C. fulvum causes leaf mould of tomato, and remains strictly apoplastic while colonising the mesophyll of the leaves (Thomma et al., 2005). The Avr4 protein contains a typical invertebrate chitin-binding domain and binds to the chitin present in fungal hyphal cell walls, thereby protecting it against hydrolysis by plant chitinases that are secreted into the apoplast in response to the attack by the fungus (de Wit, 2016; Joosten et al., 1994; Stergiopoulos and de Wit, 2009; van den Burg et al., 2006; van Esse et al., 2007). Avr9 is a small and stable cysteine-knotted peptide, structurally resembling carboxypeptidase inhibitors, but the biological function of Avr9 still remains unknown (Stergiopoulos and de Wit, 2009; Vervoort et al., 1997). Currently, whether the LRR domain of Cf-4 directly binds Avr4 remains to be elucidated, while the LRR domain of Cf-9 does not appear to directly interact with Avr9 (Luderer et al., 2001). 


\section{Complex formation between cell surface receptors and regulatory RLKs}

Upon ligand perception, both RLKs and RLPs form ligand-induced complexes with the regulatory RLK BRI1-ASSOCIATED RECEPTOR KINASE 1/SOMATIC EMBRYOGENESIS RECEPTOR KINASE 3 (BAK1/SERK3, further referred to as BAK1), or related SERKs (Gust and Felix, 2014; Liebrand et al., 2014; van der Burgh and Joosten, 2019; Wan et al., 2019). SERKs contain five LRRs in their ectodomains (Santiago et al., 2013; Sun et al., 2013a). Emerging evidence has revealed that the SERKs function as co-receptors for RLKs involved in immunity, as well as for some RLKs involved in development (Hohmann et al., 2017; Ma et al., 2016; Song et al., 2017a). For instance, the ectodomain of BAK1 directly binds flg22, which acts as a molecular glue to stabilize the heterodimerization of the ectodomains of the ligandbound FLS2 and BAK1 (Sun et al., 2013b). Currently, it remains unknown whether the SERKs also heterodimerize with ligand-bound RLPs through their ectodomains. Besides being positive regulators in immunity and development, Arabidopsis BAK1 and its closest paralogue BAK1-LIKE 1 (BKK1), which is SERK4, also function redundantly to negatively regulate plant immunity (He et al., 2007). It has been proposed that the auto-immunity in bak1 bkk1 mutants might be caused by activation of unknown NB-LRRs, which guard the integrity of BAK1 and BKK1 (Couto and Zipfel, 2016). However, the molecular mechanism of this negative role of BAK1 and BKK1 in immunity is not fully understood (Ma et al., 2016).

In addition to forming ligand-induced complexes with the SERKs, RLPs also constitutively interact with the regulatory RLK SUPPRESSOR OF BIR1-1 (SOBIR1) (Gust and Felix, 2014; Liebrand et al., 2014; van der Burgh and Joosten, 2019; Wan et al., 2019). SOBIR1 also contains five LRRs in its ectodomain (Gao et al., 2009). The ectodomain of SOBIR1 is dispensable for its constitutive interaction with various RLPs, including Cf-4, however it is required for Cf-4 signalling (Bi et al., 2016). So far, the molecular mechanism underlying the functionality of the ectodomain of SOBIR1 remains unclear. Besides being a regulatory RLK for RLPs involved in immunity, SOBIR1 is also required for the auto-immunity in a knockout mutant of BAK1INTERACTING RECEPTOR-LIKE KINASE 1 (BIR1) (Gao et al., 2009).

The Arabidopsis BIR family consists of four members, which all contain five LRRs in their ectodomain that interacts with the ectodomain of the SERKs (Gao et al., 2009; Halter et al., 2014b; Hohmann et al., 2018; Imkampe et al., 2017; Ma et al., 2017). Unlike the other BIRs, BIR1 is a kinase-active RLK and negatively regulates basal immunity (Gao et al., 2009; Halter et al., 2014b; Imkampe et al., 2017). BIR1-mediated negative regulation of immunity depends on the presence of functional SOBIR1 and BAK1 proteins, as well as on the activation of unknown NB-LRRs, but the mechanism 
by which BIR1 negatively regulates immunity remains unknown (Gao et al., 2009; Liu et al., 2016). Although BIR1 interacts with BAK1, BIR1 does not affect BAK1dependent FLS2 signalling (Gao et al., 2009; Liu et al., 2016). BIR2 and BIR3 negatively regulate immunity mediated by RLKs, including FLS2, and have been shown to sequester BAK1 from FLS2 (Halter et al., 2014b; Imkampe et al., 2017). Unlike BIR2, BIR3 also targets FLS2 to negatively regulate FLS2/BAK1 complex formation (Imkampe et al., 2017). In addition, BIR3 employs a similar mechanism to negatively regulate plant development mediated by the well-known RLK BRASSINOSTEROID INSENSITIVE1 (BRI1), which recognizes brassinosteroids (Imkampe et al., 2017; Li and Chory, 1997). Moreover, the tomato BIR3 orthologue also negatively regulates FLS2 and BRI1 signalling (Huang et al., 2017). So far, BIR4 has not been functionally characterized.

Upon ligand perception, RLKs and RLPs trigger a series of immune responses, including the production of reactive oxygen species (ROS) and the activation of mitogen-activated protein kinases (MAPKs) and $\mathrm{Ca}^{2+}$ fluxes, eventually leading to plant immunity (Bigeard et al., 2015; Couto and Zipfel, 2016; Yu et al., 2017; Zhou et al., 2019). In this chapter, an overview of early signalling events occurring in RLK- and RLP-mediated immunity will be provided, including examples of such events taking place in FLS2 and Cf-4 signalling, respectively. Essentially, the extensive work that has been done on deciphering FLS2 signalling is anticipated to provide leads for understanding how $\mathrm{Cf}-4$ signalling takes place.

\section{RLKs in plant immunity}

In addition to FLS2 and EFR, many other RLKs have been demonstrated to mediate immunity against infection by different pathogens in various plant species (Boutrot and Zipfel, 2017). For instance, FLS3 and COLD SHOCK PROTEIN RECEPTOR (CORE) mediate immunity against bacterial infection in Solanaceous plants upon recognition of flgll-28, which is a flagellin epitope different from flg22, and bacterial cold-shock proteins, respectively (Hind et al., 2016; Wang et al., 2016a). In addition, the wellknown rice RLK XA21 recognizes the 21-amino-acid peptide RaxX21 of REQUIRED FOR ACTIVATION OF XA21 (RaxX), which is derived from the bacterial pathogen Xanthomonas oryzae pv. oryzae, to activate immunity (Pruitt et al., 2015; Song et al., 1995). Furthermore, the Arabidopsis RLKs PROPEP1 RECEPTOR $1 / 2$ (PEPR1/2) and RLK7, and the tomato SYSTEMIN RECEPTOR1 (SYR1), recognize endogenous peptides to trigger plant immunity (Hou et al., 2014; Krol et al., 2010; Wang et al., 2018b; Yamaguchi et al., 2010; Yamaguchi et al., 2006).

Upon ligand perception, RLKs form complexes with SERKs to initiate downstream 
signalling (Ma et al., 2016; Wan et al., 2019). Emerging evidence has revealed that receptor-like cytoplasmic kinases (RLCKs) are direct phosphorylation substrates of activated RLK/SERK complexes (Cui et al., 2018; Liang and Zhou, 2018). Subsequently, RLCKs initiate the activation of various downstream immune responses, including ROS production and MAPK activation (Cui et al., 2018; Liang and Zhou, 2018).

So far, FLS2-mediated immunity against bacterial infection has been extensively studied and various components involved in FLS2-mediated early immune signalling have been reported (Couto and Zipfel, 2016; Mithoe and Menke, 2018; Wan et al., 2019; Zhou et al., 2019). To suppress host immunity for successful colonization, bacterial pathogens inject a set of effectors into the plant cells through their type III secretion system (T3SS) (Büttner and He, 2009). For instance, the model bacterial pathogen Pseudomonas syringae pv. tomato (Pst) DC3000 delivers at least 28 T3SS effectors (T3Es) into the cells of tomato, Nicotiana benthamiana and Arabidopsis, and some T3Es have been demonstrated to suppress FLS2 signalling in different ways (Büttner, 2016; Cunnac et al., 2009; Lee et al., 2013; Xin and He, 2013). Here, an overview of early immune signalling events occurring upon activation of FLS2 signalling will be provided, together with an overview of bacterial T3E-mediated suppression of FLS2 signalling.

Regulation of the signalling activity of the FLS2/BAK1 complex at the plasma membrane

Both the ectodomains of FLS2 and BAK1 directly bind flg22, which induces and stabilizes FLS2/BAK1 heterodimerization (Chinchilla et al., 2007; Schulze et al., 2010; Sun et al., 2013b) (Fig. 1). In addition, binding of flg22 triggers rapid transphosphorylation of the intracellular kinase domains that have come in close proximity, leading to the activation of the FLS2/BAK1 complex (Schulze et al., 2010) (Fig. 1, right panel).

The flg22-induced FLS2/BAK1 heterodimerization and subsequent activation is under strict regulation (Couto and Zipfel, 2016; Wan et al., 2019). So far, various plasma membrane-associated proteins have been revealed to either positively or negatively regulate the flg22-induced FLS2/BAK1 complex formation (Wan et al., 2019). For instance, the RLKs BIR2 and BIR3 sequester BAK1 from FLS2, while perception of flg22 triggers the dissociation of BIR2 and BIR3 from BAK1 that subsequently associates with FLS2 (Halter et al., 2014b; Imkampe et al., 2017) (Fig. 1). In addition to targeting BAK1, BIR3 also directly interacts with FLS2 to negatively regulate FLS2/BAK1 complex formation (Imkampe et al., 2017). Besides regulation of complex 
formation, the phosphorylation status of FLS2 and BAK1 is also controlled by protein phosphatases. For instance, the protein phosphatase type 2C (PP2C) subunit KINASE ASSOCIATED PROTEIN PHOSPHATASE (KAPP) interacts with the kinase domain of FLS2 and negatively regulates flg22-triggered immune responses (Gómez-Gómez et al., 2001). In addition, the serine/threonine protein phosphatase type 2A (PP2A) subunits A1, B' $\eta$, B' $\zeta$ and C4 constitutively interact with the kinase domain of BAK1 and negatively regulate its kinase activity (Segonzac et al., 2014) (Fig. 1). Furthermore, the plant U-box (PUB) E3 ubiquitin ligases PUB12 and PUB13 constitutively interact with the kinase domain of BAK1, and activated BAK1 phosphorylates these two ubiquitin ligases that subsequently target and ubiquitinate FLS2 for degradation (Lu et al., 2011) (Fig. 1).

\section{Regulation of the flg22-triggered ROS burst}

The plasma membrane-associated NADPH oxidase RESPIRATORY BURST OXIDASE HOMOLOG D (RBOHD) is the key enzyme mediating ROS production in FLS2 signalling (Kadota et al., 2014; Li et al., 2014b) (Fig. 1). So far, various components have been revealed to be involved in this process. For instance, the wellstudied RLCK BOTRYTIS-INDUCED KINASE1 (BIK1) constitutively interacts with the kinase domains of both FLS2 and BAK1 (Lin et al., 2014; Lu et al., 2010; Zhang et al., 2010) (Fig. 1, left panel). Upon flg22 perception, activated BAK1 phosphorylates BIK1, which trans-phosphorylates and subsequently dissociates from the FLS2/BAK1 complex (Lin et al., 2014; Lu et al., 2010; Zhang et al., 2010) (Fig. 1, right panel). Activated BIK1 then phosphorylates RBOHD to trigger ROS production (Kadota et al., 2014; Li et al., 2014b) (Fig. 1, right panel). The flg22-triggered ROS burst is also regulated by the heterotrimeric $\mathrm{G}$-protein complex, which consists of the Ga-containing subunits EXTRA-LARGE G-PROTEIN 2 (XLG2) and XLG3, the G-protein $\beta$ subunit AGB1 and the G-protein y subunits AGG1 and AGG2 (Liang et al., 2016) (Fig. 1). XLG2 interacts with the FLS2/BIK1 complex and dissociates from both FLS2 and BIK1 upon its phosphorylation by activated BIK1 (Liang et al., 2016). In addition, XLG2 also constitutively interacts with RBOHD and might also phosphorylate this enzyme to initiate ROS production (Liang et al., 2016). Moreover, the plasma membraneassociated GTPase-accelerating protein REGULATOR OF G-PROTEIN SIGNALING 1 (RGS1) constitutively interacts with FLS2 and XLG2 to stabilize the heterotrimeric Gprotein complex through inactivating XLG2 (Liang et al., 2018) (Fig. 1, left panel). Furthermore, upon flg22 perception, RGS1 is phosphorylated in a BIK1-dependent manner, after which it dissociates from FLS2 and XLG2, resulting in the activation and subsequent dissociation of the G-protein complex (Liang et al., 2016; Liang et al., 2018) (Fig. 1, right panel). 


\section{Chapter 1}

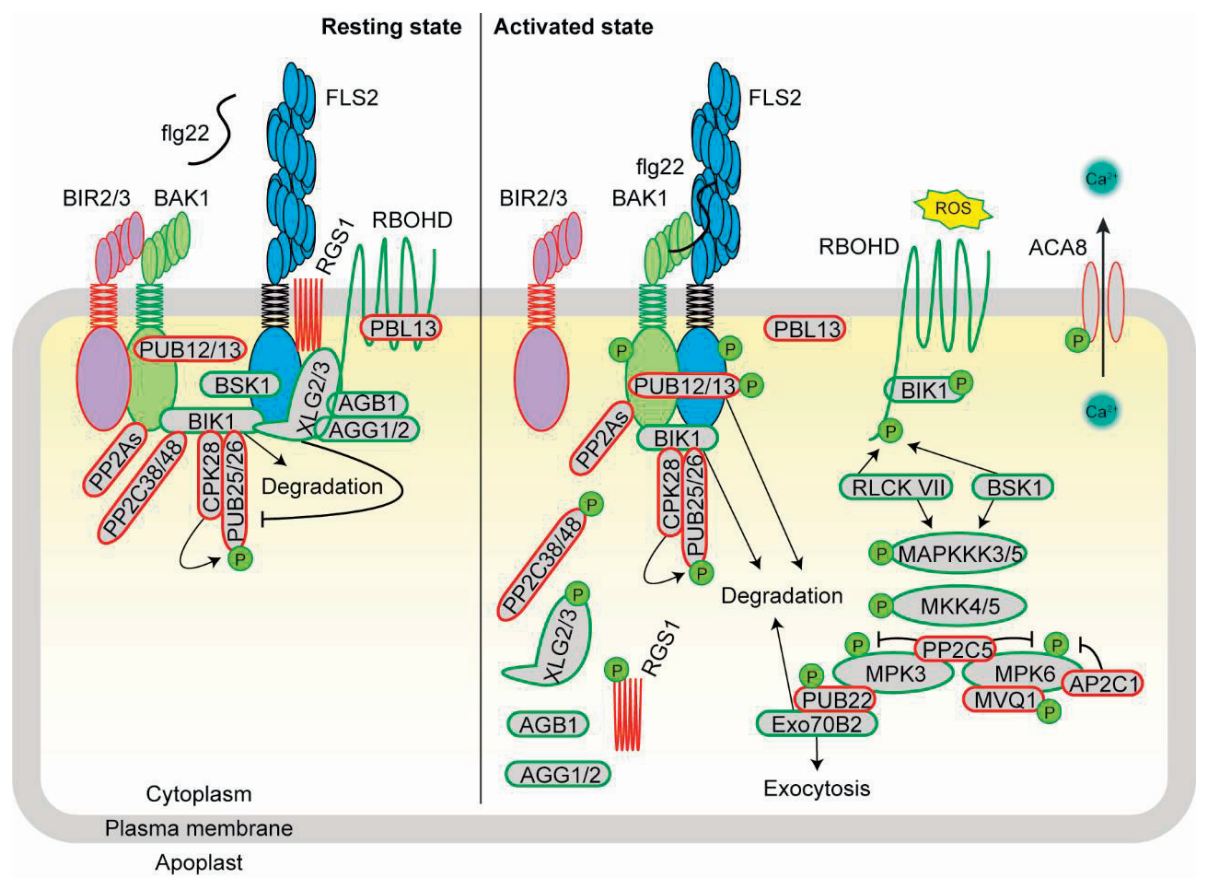

Figure 1. Overview of the regulators involved in FLS2-mediated early immune signalling in Arabidopsis thaliana. The plasma membrane-associated receptor FLS2 recognizes the bacterial flagellin epitope flg22 to heterodimerize with its co-receptor BAK1 and relies on a set of regulators to activate downstream immune responses. In the resting state (left panel), the RLKs BIR2 and BIR3 sequester BAK1 from FLS2 to avoid FLS2/BAK1 complex formation in the absence of flg22. The protein phosphatase PP2A subunits (PP2As) constitutively interact with BAK1 and dephosphorylate this coreceptor to keep it at a low active state. Both FLS2 and BAK1 interact with the RLCK BIK1. The activity of BIK1 is negatively regulated by the protein phosphatases PP2C38 and PP2C48, which interact with the FLS2/BIK1 complex. In addition, the accumulation of the BIK1 protein is controlled by the E3 ubiquitin ligases PUB25 and PUB26. The activity of PUB25/26 is stimulated by the $\mathrm{Ca}^{2+}$ sensor CPK28, whereas this activity is suppressed by the heterotrimeric G-protein complex, which consists of the Gacontaining subunits $X L G 2$ and $X L G 3$, the $G \beta$ subunit $A G B 1$ and the $G y$ subunits $A G G 1$ and $A G G 2$. Moreover, the GTPase accelerating protein RGS1 associates with FLS2 and the XLGs, and inactivates the activity of the latter to stabilize the heterotrimeric G-protein complex. Furthermore, the plasma membrane-associated RLCK PBL13 prevents inappropriate activation of immune responses through interacting with RBOHD, which is the main enzyme mediating reactive oxygen species (ROS) production in the FLS2 signalling pathway. In the activated state (right panel), flg22 perception triggers the dissociation of BIR2 and BIR3 from BAK1, the latter which subsequently heterodimerizes with FLS2 through their ectodomains, leading to phosphorylation of the FLS2/BAK1 complex. Activated BAK1 phosphorylates the E3 ubiquitin ligases PUB12 and PUB13, which subsequently target and ubiquitinate FLS2 for degradation. In addition, activated BAK1 also phosphorylates BIK1, which transphosphorylates, and subsequently dissociates, from the FLS2/BAK1 complex. Activated BIK1 then phosphorylates RBOHD to initiate ROS production. In addition, activated BIK1 also phosphorylates PP2C38 and PP2C48, resulting in their dissociation from BIK1. Perception of flg22 also leads to the dissociation of the heterotrimeric G-protein complex. Furthermore, perception of flg22 triggers enhanced CPK28-dependent phosphorylation levels of PUB25 and PUB26, which specifically ubiquitinate non- 
activated BIK1 for degradation, thereby controlling the amplitude of the immune responses. Besides BIK1, additional RLCK VII family members, as well as the RLCK XII family member BSK1, which dissociates from FLS2 upon flg22 perception, are also required for the flg22-triggered ROS burst. In addition, these RLCK VII family members and BSK1 also interact with MAPKKK5 and activate the MAPK cascade MAPKKK3/5-MKK4/5-MPK3/6. MPK6 phosphorylates MVQ1, while MPK3 phosphorylates PUB22, leading to inhibition of the auto-ubiquitination and degradation of this E3 ligase. Stabilized PUB22 in turn promotes ubiquitination and degradation of Exo70B2, thereby attenuating FLS2 signalling Moreover, the protein phosphatase AP2C1 predominantly suppresses phosphorylation of MPK6, while another protein phosphatase PP2C5 dephosphorylates both MPK3 and MPK6. Furthermore, perception of flg22 triggers phosphorylation of ACA8, which promotes $\mathrm{Ca}^{2+}$ efflux to negatively regulate FLS2 signalling. Note that not all regulators mentioned in this chapter are indicated due to space limitations (see text for further details). Positive and negative regulators of FLS2 signalling are indicated by green and red boxes, respectively. The "P" placed in a green circle represents phosphorylation.

Since BIK1 plays a critical role in mediating signal transduction downstream of the FLS2/BAK1 complex, accumulation and activation of BIK1 are under strict control to avoid deregulated activation of plant immunity (Wan et al., 2019; Zhou et al., 2019). The E3 ubiquitin ligases PUB25 and PUB26 (further referred to as PUB25/26) ubiquitinate non-activated BIK1 for degradation to negatively regulate FLS2 signalling (Wang et al., 2018a) (Fig. 1). The activity of PUB25/26 is stimulated by the $\mathrm{Ca}^{2+}$ sensor CALCIUM-DEPENDENT PROTEIN KINASE 28 (CPK28) through phosphorylation, whereas this activity is inhibited by the heterotrimeric G-protein complex (Liang et al., 2016; Monaghan et al., 2014; Wang et al., 2018a) (Fig. 1). Furthermore, the protein phosphatase PP2C38, which functions redundantly with PP2C48, constitutively interacts with the FLS2/BIK1 complex to dephosphorylate BIK1 (Couto et al., 2016) (Fig. 1, left panel). Upon flg22 perception, activated BIK1 phosphorylates these phosphatases, leading to their dissociation from both FLS2 and BIK1 (Couto et al., 2016) (Fig. 1, right panel).

The Arabidopsis RLCK VII family consists of 46 members that are subdivided into nine clades (Rao et al., 2018). In addition to the RLCK VII-8 clade member BIK1, various additional members of the RLCK VII family also play a role in the flg22-triggered ROS burst. For instance, AVRPPHB SUSCEPTIBLE 1 (PBS1), PBS1-LIKE 1 (PBL1) and PBL2, which are members of RLCK VII-1, VII-8 and VII-9 clades, respectively, are all required for this process (Zhang et al., 2010). Recently, it has also been shown that members of the RLCK VII-5, -7 and -8 clades are redundantly required for the activation of the flg22-triggered ROS burst, whereas members of the RLCK VII-6 clade function redundantly to negatively regulate this process (Bi et al., 2018; Rao et al., 2018). In addition, the plasma membrane-associated VII-6 clade member PBL13 dynamically interacts with RBOHD to negatively regulate the flg22-triggered ROS burst 
(Lin et al., 2015) (Fig. 1). Furthermore, the flg22-triggered ROS burst also depends on members of the other RLCK families. For instance, the RLCK XII family member BRASSINOSTEROID-SIGNALING KINASE 1 (BSK1), which dissociates from FLS2 upon flg22 perception, is required for the flg22-triggered ROS burst (Shi et al., 2013) (Fig. 1). In addition, the tomato RLCK VIII family members PTO-INTERACTING PROTEIN1a (PTI1a) and PTI1b are also required for this response (Schwizer et al., 2017).

\section{MAPK cascades and their regulators required for FLS2 signalling}

A MAPK cascade generally consists of three tiers of protein kinases, namely MAP kinase kinase kinases (MAPKKKs or MEKKs), MAP kinase kinases (MKKs) and MAP kinases (MPKs) (Zhang and Klessig, 2001). In addition, activation of such MAPK cascades plays an important role in immunity triggered by plasma membraneassociated receptor complexes (Meng and Zhang, 2013). So far, it is known that the MAPK cascades MAPKKK3/5-MKK4/5-MPK3/6 (Fig. 1, right panel) and MEKK1MKK1/2-MPK4 are required for FLS2 signalling (Asai et al., 2002; Bi et al., 2018; Gao et al., 2008; Zhang et al., 2012). In addition, the MAPKKK ENHANCED DISEASE RESISTANCE 1 (EDR1) negatively regulates FLS2 signalling, probably through its interaction with MKK4/5 that results in reduced kinase activity and protein levels of downstream MPK3/6 (Geissler et al., 2015; Zhao et al., 2014). Moreover, MAPKKK7 negatively regulates flg22-triggered MAPK activation probably through competing with MAPKKK3/5 for binding of MKK4/5 (Mithoe et al., 2016).

Emerging evidence indicates that RLCKs function redundantly to activate MAPKKKs downstream of activated receptor complexes (Cui et al., 2018; Liang and Zhou, 2018). For example, various RLCK VII family members, including BIK1, interact with MAPKKK5 and redundantly regulate the activation of this MAPKKK in FLS2 signalling (Bi et al., 2018; Kong et al., 2016) (Fig. 1, right panel). In addition, the RLCK XII family member BSK1, which dissociates from FLS2 upon flg22 perception, directly targets and phosphorylates MAPKKK5 (Shi et al., 2013; Yan et al., 2018) (Fig. 1). Furthermore, FLS2 itself also interacts with MAPKKK7, and phosphorylation of this MAPKKK in turn negatively regulates FLS2 signalling (Mithoe et al., 2016).

Beside MAPK activators, some MAPK substrates required for FLS2 signalling have also been reported (Zhang et al., 2018). For instance, MPK3/6-TARGETED VQP 1 (MVQ1), which belongs to a class of proteins containing $V Q$ motifs and interacts with various WRKY transcription factors, is directly phosphorylated by MPK6 when FLS2 is activated (Pecher et al., 2014) (Fig. 1, right panel). Another example is the E3 ubiquitin ligase PUB22, which functions redundantly with PUB23 and PUB24 to negatively 
regulate FLS2 signalling (Trujillo et al., 2008) (Fig. 1, right panel). Phosphorylation of PUB22 mediated by MPK3 inhibits the auto-ubiquitination and degradation of this E3 ligase (Furlan et al., 2017). Stabilized PUB22 in its turn promotes degradation of its ubiquitination substrate EXOCYST SUBUNIT EXO70 FAMILY PROTEIN B2 (Exo70B2), which is involved in exocytosis and is a positive regulator of FLS2 signalling (Pecenkova et al., 2011; Stegmann et al., 2012) (Fig. 1, right panel).

Protein phosphatase-mediated dephosphorylation of MAPK cascades is a major determinant in controlling plant immunity (Meng and Zhang, 2013). The PP2C subunits AP2C1 and PP2C5 have been demonstrated to negatively regulate FLS2 signalling through their interactions with various MPKs. For instance, AP2C1 predominantly interacts with MPK6 and suppresses the flg22-induced phosphorylation of MPK6 (Galletti et al., 2011; Schweighofer et al., 2007; Shubchynskyy et al., 2017) (Fig. 1, right panel). In addition, PP2C5 dephosphorylates MPK3 and MPK6 (Fig. 1, right panel), as well as MPK4 (Brock et al., 2010).

\section{$\mathrm{Ca}^{2+}$-dependent regulation of FLS2 signalling}

$\mathrm{Ca}^{2+}$-dependent signalling plays an essential role in plant immunity (Yuan et al., 2017). It is for example known that perception of flg22 triggers BAK1- and BIK1-dependent elevated levels of cytosolic $\mathrm{Ca}^{2+}$, which in turn either positively or negatively regulate FLS2 signalling through $\mathrm{Ca}^{2+}$ sensors (Li et al., 2014b; Ma et al., 2013; Ranf et al., 2014). The $\mathrm{Ca}^{2+}$ sensor CPK28 phosphorylates PUB25/26 to enhance their E3 ligase activity, which in turn degrade non-activated BIK1 to negatively regulate FLS2 signalling (Monaghan et al., 2014; Wang et al., 2018a) (Fig. 1). In addition, various CPKs are required for the flg22-triggered ROS burst, and CPK5 has been shown to promote phosphorylation of ROBHD to regulate the output of the FLS2 signalling pathway (Boudsocq et al., 2010; Dubiella et al., 2013).

The homeostasis of the cytosolic $\mathrm{Ca}^{2+}$ level is balanced by $\mathrm{Ca}^{2+}$ influx channels and $\mathrm{Ca}^{2+}$ efflux transporters (Tang and Luan, 2017). The tomato $\mathrm{Ca}^{2+}$ influx channels CYCLIC NUCLEOTIDE-GATED CHANNEL 16 (CNGC16) and CNGC18 contribute to the flg22-triggered ROS production (Saand et al., 2015). However, it has also been shown that Arabidopsis CNGC2, which is the orthologue of tomato CNGC16, is not required for the flg22-triggered $\mathrm{Ca}^{2+}$ influx (Jeworutzki et al., 2010). Concerning the regulation of the $\mathrm{Ca}^{2+}$ efflux related to $\mathrm{FLS} 2$ signalling, the plasma membraneassociated $\mathrm{Ca}^{2+}$ efflux transporter AUTOINHIBITED Ca ${ }^{2+}$-ATPase 8 (ACA8) interacts with FLS2 (Frei dit Frey et al., 2012) and functions redundantly with its paralogues to negatively regulate FLS2 signalling by lowering the cytosolic $\mathrm{Ca}^{2+}$ level (Yang et al., 2017; Yu et al., 2018). In addition, flg22 perception triggers phosphorylation of ACA8 
(Kadota et al., 2019) (Fig. 1, right panel). So far, the molecular mechanism by which ACA8 regulates the cytosolic $\mathrm{Ca}^{2+}$ level downstream of FLS2 remains to be elucidated.

\section{Suppression of FLS2-mediated early immune signalling mediated by bacterial T3ES}

To successfully colonize their host plants, bacterial pathogens secrete a set of T3Es into their host cells to suppress plant immunity. So far, various T3Es of Pst DC3000 and additional bacterial pathogens have been shown to suppress FLS2 signalling in different ways (Büttner, 2016; Lee et al., 2013).

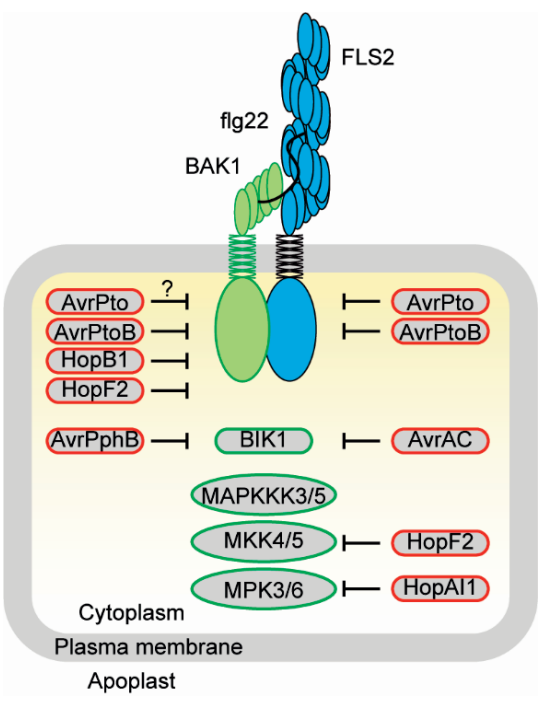

Figure 2. Bacterial T3Es suppress FLS2-mediated early immune signalling. Bacterial T3Es can target the flg22-induced FLS2/BAK1 complex and the various downstream components to suppress FLS2 signalling (see text for details). Note that the T3Es AvrPphB and AvrAC are from Pseudomonas syringae pv. phaseolicola and Xanthomonas campestris pv. campestris, respectively, and that the other T3Es are from $P$. syringae pv. tomato DC3000. Positive and negative regulators of FLS2 signalling are indicated by green and red boxes, respectively. The question mark indicates contradicting reports.

Various T3Es have been demonstrated to target FLS2, BAK1 and BIK1, thereby suppressing FLS2 signalling (Büttner, 2016; Couto and Zipfel, 2016) (Fig. 2). The wellknown Pst DC3000 T3E AvrPto targets the kinase domain of FLS2 and suppresses phosphorylation of FLS2 and BIK1 (Xiang et al., 2008; Zhang et al., 2010). In addition, AvrPto targets FLS2 and, possibly, BAK1 to inhibit FLS2/BAK1 complex formation (Shan et al., 2008; Xiang et al., 2011; Xiang et al., 2008; Zhou et al., 2014) (Fig. 2). Another example is the Pst DC3000 T3E AvrPtoB, which possesses E3 ubiquitin ligase activity to ubiquitinate FLS2 for degradation, and targets both FLS2 and BAK1 to inhibit FLS2/BAK1 complex formation (Cheng et al., 2011; Göhre et al., 2008; Shan et al., 2008) (Fig. 2). In addition, the Pst DC3000 T3E HopB1 is a serine protease that associates with FLS2 and specifically cleaves activated BAK1 to suppress FLS2 signalling (Li et al., 2016b) (Fig. 2). Besides HopB1, the Pst DC3000 T3E HopF2, which is a mono-ADP-ribosyltransferase that hydrolyses $\mathrm{NAD}^{+}$to transfer $\mathrm{ADP}$ ribose to its 
substrate (Deng and Barbieri, 2008), also targets BAK1 and suppresses phosphorylation of BIK1, probably through ADP-ribosylating BAK1 (Wu et al., 2011; Zhou et al., 2014) (Fig. 2). Furthermore, both the $P$. syringae pv. phaseolicola T3E AvrPphB, which is a cysteine protease, and the $X$. campestris pv. campestris T3E AvrAC, which is an uridylyl transferase, target multiple RLCK VII family members to suppress FLS2 signalling. For instance, AvrPphB cleaves BIK1 and AvrAC uridylylates BIK1 to inhibit its phosphorylation (Feng et al., 2012; Zhang et al., 2010) (Fig. 2).

The MAPK cascade is also a hub for Pst DC3000 T3Es to suppress FLS2 signalling (Bi and Zhou, 2017). In addition to targeting BAK1, HopF2 predominantly targets MKK4 and MKK5, and ADP-ribosylates MKK5 to suppress activation of downstream MPKs (Wang et al., 2010c) (Fig. 2). In addition, the phosphothreonine lyase HopAl1 dephosphorylates MPK3 and MPK6 (Fig. 2), as well as MPK4, to suppress FLS2 signalling (Zhang et al., 2007; Zhang et al., 2012).

\section{RLPs in plant immunity}

Tomato Cf-4 and Cf-9 are two well-studied RLPs that confer resistance to the biotrophic fungal pathogen C. fulvum secreting the effectors Avr4 and Avr9, respectively (Jones et al., 1994; Joosten et al., 1994; Thomas et al., 1997; van den Ackerveken et al., 1992). In addition, Cf-2, Cf-4E and Cf-5 also confer resistance to C. fulvum through recognition of their matching effectors, namely Avr2, Avr4E and Avr5 (Dixon et al., 1998; Dixon et al., 1996; Luderer et al., 2002; Mesarich et al., 2016; Takken et al., 1999; Westerink et al., 2004). Besides Cf proteins, many additional tomato RLPs mediating plant immunity have also been identified. For instance, Ve1 confers resistance to the fungal pathogen Verticillium dahliae secreting the effector Ave1 (de Jonge et al., 2012). In addition, the I protein recognizes the effector Avr1 to mediate immunity against infection by the fungal pathogen Fusarium oxysporum f. sp. Iycopersici (Catanzariti et al., 2017). Moreover, ETHYLENE-INDUCING XYLANASE RECEPTOR 2 (EIX2) recognizes the MAMP ETHYLENE-INDUCING XYLANASE (EIX) to activate immunity (Ron and Avni, 2004). Furthermore, RLP-mediated immunity has also been demonstrated in other plant species, including potato, $N$. benthamiana and Arabidopsis (Albert et al., 2015; Boutrot and Zipfel, 2017; Du et al., 2015; van der Burgh and Joosten, 2019; Wan et al., 2019; Wang et al., 2018c; Zhang et al., 2014; Zhang et al., 2013a). For instance, Arabidopsis RLP23 mediates defence against infection by pathogens producing NECROSIS- AND ETHYLENE-INDUCING PROTEIN 1 (NEP1)like proteins (NLPs) (Albert et al., 2015; Böhm et al., 2014b; Oome et al., 2014).

Immunity mediated by the two gene-for-gene pairs Cf-4/Avr4 and Cf-9/Avr9 has been studied extensively (de Wit, 2016; Stergiopoulos and de Wit, 2009). The combination 
of Cf-4/Avr4 and Cf-9/Avr9 both activate downstream defence signalling eventually leading to the development of an HR in tomato, as well as in $N$. tabacum and $N$. benthamiana when heterologously expressed (Gabriëls et al., 2006; HammondKosack et al., 1994; Hammond-Kosack et al., 1998; Joosten et al., 1994; Thomas et al., 1997; Thomas et al., 2000). The other Cf/Avr combinations and many other RLP/ligand pairs also trigger an HR, whereas not all RLPs, such as RLP23, recognize their ligands to trigger an HR (Albert et al., 2015; van der Burgh and Joosten, 2019).

RLPs require SOBIR1 and BAK1 to initiate downstream immune signalling (Gust and Felix, 2014; Liebrand et al., 2014; van der Burgh and Joosten, 2019; Wan et al., 2019). However, the downstream components required for RLP-mediated immunity are barely known. Nevertheless, upon recognition of their ligands, some RLPs, including Cf-4, Cf9 and RLP23, trigger ROS production and the activation of MAPKs and $\mathrm{Ca}^{2+}$ signalling, which is reminiscent of the activation of FLS2-mediated signalling (Albert et al., 2015; Couto and Zipfel, 2016; de Jong et al., 2004; Romeis et al., 2001; Stulemeijer et al., 2007; Wan et al., 2018; Zhang et al., 2013a; Zhou et al., 2019). Below, an overview of identified downstream signalling components required for Cf-4 signalling will be provided, with a focus on the following aspects: the activation of the Cf4/SOBIR1/BAK1 complex; the cytoplasmic interactors of Cf-4; the requirement of downstream MAPKs and the regulation of Cf-4 signalling mediated by $\mathrm{Ca}^{2+}$-dependent signalling and E3 ubiquitin ligases (Fig. 3).

\section{Formation and activation of the Cf-4/SOBIR1/BAK1 complex}

Cf-4 requires the RLKs SOBIR1 and BAK1 to mediate resistance to $C$. fulvum (Liebrand et al., 2013; Postma et al., 2016). The transmembrane domain of SOBIR1 is responsible for its constitutive interaction with $\mathrm{Cf}-4$, and BAK1 is recruited to the constitutive Cf-4/SOBIR1 complex upon Avr4 perception (Bi et al., 2016; Liebrand et al., 2013; Postma et al., 2016) (Fig. 3A). In addition, the kinase activities of both SOBIR1 and BAK1 are required Cf-4 signalling (Liebrand et al., 2013; Postma et al., 2016). Recently, it has been proposed that formation of the Cf-4/SOBIR1/BAK1 complex initiates trans-phosphorylation events between the kinase domains of SOBIR1 and BAK1 that have come in close proximity, leading to full activation of this complex and subsequent activation of downstream signalling (van der Burgh et al., 2019).

\section{Cytoplasmic interactors of $\mathrm{Cf}-4$}

The Cf-4 and Cf-9 proteins share an identical C-terminal half, including the transmembrane domain and the cytoplasmic tail (Jones et al., 1994; Thomas et al., 1997). The cytoplasmic tail of Cf-4/9 interacts with tomato VESICLE-ASSOCIATED 
PROTEIN 27 (VAP27), which is proposed to regulate vesicular trafficking and might be required for the Avr4-triggered endocytosis of Cf-4 (Chakrabarti et al., 2016; Laurent et al., 2000; Postma et al., 2016). The cytoplasmic tail of Cf-4/9 also interacts with the tomato thioredoxin protein Cf-9-INTERACTING THIOREDOXIN (CITRX), and the binding sites for VAP27 and CITRX on this cytosolic tail appear to be different (Chakrabarti et al., 2016; Rivas et al., 2004) (Fig. 3B). Both CITRX and its $N$. benthamiana orthologue are required for Cf-9 signalling (Rivas et al., 2004), suggesting that CITRX might be required for $\mathrm{Cf}-4$ signalling as well. CITRX functions as an adaptor protein between the cytoplasmic tail of $\mathrm{Cf}-4 / 9$ and the tomato RLCK Avr9/Cf-9 INDUCED KINASE 1 (ACIK1) (Nekrasov et al., 2006; Rowland et al., 2005) (Fig. 3B). AClK1 is required for the Cf-4/Avr4-triggered HR in N. benthamiana, as well as for Cf-9 signalling in tomato and $N$. benthamiana (Rowland et al., 2005).

\section{MAPKs are required for $\mathrm{Cf}-4$ signalling}

It has been reported that NbMAPKKKE, the orthologue of $S / M A P K K K \varepsilon$, is required for the Cf-4/Avr4-triggered HR (Melech-Bonfil and Sessa, 2010). In addition, SIMPK1, SIMPK2 and SIMPK3 (further referred to as S/MPK1/2/3) are required for Cf-4 signalling in tomato (Stulemeijer et al., 2007). Moreover, the S/MKK2 and S/MPK1/2/3 functional orthologues of $N$. benthamiana function downstream of $N b M A P K K K \varepsilon$ (Melech-Bonfil and Sessa, 2010). Taken together, it is likely that a MAPK cascade consisting of SIMAPKKKE, S/MKK2 and S/MPK1/2/3 is required for $\mathrm{Cf}-4$ signalling (Fig. 3C).

\section{$\mathrm{Ca}^{2+}$-dependent regulation of $\mathrm{Cf}-4$ signalling}

So far, little is known about $\mathrm{Ca}^{2+}$-dependent signalling playing a role downstream of $\mathrm{Cf}-4$, except that the $\mathrm{Ca}^{2+}$ sensor $\mathrm{NbCPK} 2$ is required for the HR triggered by Cf-4/Avr4 (Fig. 3D) and Cf-9/Avr9 (Romeis et al., 2001). Since the endoplasmic reticulumlocalized $\mathrm{Ca}^{2+}$ efflux transporter $N$. benthamiana $\mathrm{Ca}^{2+}$-ATPase (NbCA1) negatively regulates the Cf-9/Avr9-triggered HR (Zhu et al., 2010a), NbCA1 might also negatively regulate Cf-4 signalling (Fig. 3D). NbCPK2 and NbCA1 are the orthologues of Arabidopsis CPK1/2 and ACA2, respectively (Cheng et al., 2002; Romeis et al., 2001; Zhu et al., 2010a). The activity of ACA2 is stimulated by the $\mathrm{Ca}^{2+}$ sensor protein CALMODULIN (CaM), whereas CPK1 phosphorylates ACA2 to inhibit the CaMmediated stimulation of ACA2 (Harper et al., 1998; Hwang et al., 2000). Hence, it is likely that $\mathrm{NbCPK} 2$ and an unknown CaM member antagonistically regulate the activity of $\mathrm{NbCA} 1$ to regulate $\mathrm{Cf}-4$ signalling when expressed in $N$. benthamiana (Fig. 3D). 


\section{Chapter 1}

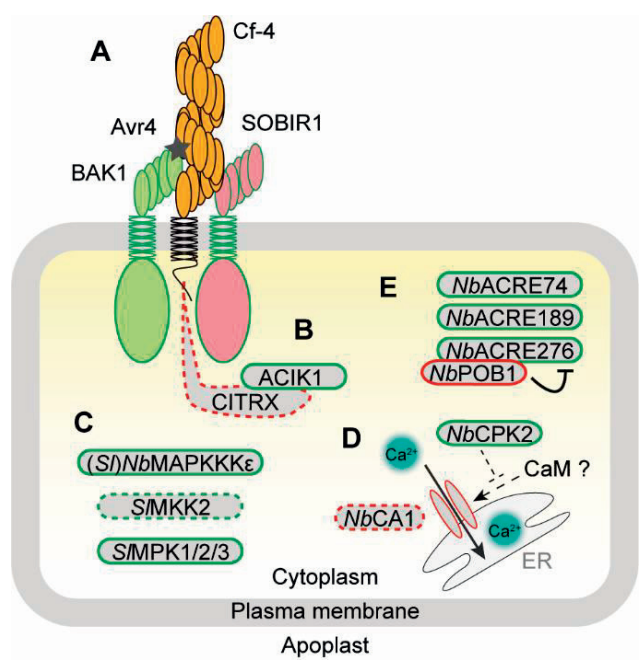

Figure 3. Overview of signalling components required for Cf-4 signalling. (A) The Avr4-induced Cf-4/SOBIR1/BAK1 complex at the plasma membrane. Note that Cf-4 constitutively interacts with SOBIR1, and BAK1 is recruited to the constitutive Cf-4/SOBIR1 complex upon recognition of Avr4. In addition, Cf-4 recognizes Avr4 to trigger the development of a hypersensitive response (HR) in both tomato (Solanum lycopersicum) and Nicotiana benthamiana. (B) The thioredoxin protein CITRX functions as an adaptor between the cytoplasmic tail of Cf-4 and the RLCK ACIK1. Note that the cytoplasmic tail of Cf- 4 is identical to that of Cf-9. ACIK1 is required for both Cf-4 and Cf-9 signalling, while CITRX might negatively regulates Cf-4 signalling as it negative regulates Cf-9 signalling. (C) SIMAPKKKE, SIMKK2 and SIMPK1/2/3 might form a MAPK cascade that is required for Cf-4 signalling. NbMAPKKKE, the orthologue of SIMAPKKKE, and S/MPK1/2/3 are required for the Cf-4/Avr4-triggered $\mathrm{HR}$ in $N$. benthamiana and tomato, respectively. In addition, the functional orthologues of SIMKK2 and SIMPK $1 / 2 / 3$ of $N$. benthamiana function downstream of NbMAPKKKE. (D) The $\mathrm{Ca}^{2+}$ sensors $N b C P K 2$ and an unknown CaM member might antagonistically regulate the activity of the endoplasmic reticulum (ER)-localized $\mathrm{Ca}^{2+}$ efflux transporter $N b C A 1$ to regulate Cf-4 signalling. Note that $N b C P K 2$ is required for both Cf- 4 and $\mathrm{Cf}-9$ signalling, while NbCA1 might negatively regulate Cf-4 signalling as it negatively regulates $\mathrm{Cf}-9$ signalling. In addition, NbCPK2 and NbCA1 are the orthologues of AtCPK1 and AtACA2, respectively, and AtCPK1 suppresses the $\mathrm{Ca}^{2+}$ sensor CaM-mediated activation of AtACA2. (E) Four E3 ubiquitin ligases regulate Cf-4 signalling in $N$. benthamiana. The E3 ubiquitin ligases ACRE74, ACRE189 and ACRE276 are required for the Cf-4/Avr4-triggered HR, while the E3 ubiquitin ligase POB1 ubiquitinates ACRE276 for degradation and negatively regulates Cf-4 signalling. Positive and negative regulators of Cf-4 signalling are indicated by green and red boxes, respectively. Possible regulatory roles and proposed functions are indicated by dashed boxes and dashed lines, respectively (see text for details).

\section{Cf-4 signalling is regulated by E3 ubiquitin ligases}

Four E3 ubiquitin ligases have been shown to regulate Cf-4 signalling in $N$. benthamiana (Fig. 3E). The E3 ubiquitin ligases Avr9/Cf-9 RAPIDLY ELICITED 74 (ACRE74), ACRE189 and ACRE276 are required for the Cf-4/Avr4-triggered HR (Gilroy et al., 2011; González-Lamothe et al., 2006; Rowland et al., 2005; van den Burg 
et al., 2008; Yang et al., 2006). However, targets of these three E3 ubiquitin ligases remain unknown. Recently, the E3 ubiquitin ligase POZ/BTB CONTAINING GPROTEIN 1 (POB1) has been shown to ubiquitinate ACRE276 for degradation, thereby negatively regulating Cf-4 signalling (He et al., 2015; Orosa et al., 2017).

\section{Searching for candidates required for Cf-4 signalling: A lesson from the well- characterized FLS2 signalling pathway and bacterial T3E-mediated suppression of FLS2 signalling}

Although cell surface receptors recognize different ligands to confer immunity against infection by a broad range of pathogens in various plant species, emerging evidence has revealed some common themes for such receptors in activating plant immunity (Bigeard et al., 2015; Couto and Zipfel, 2016; Yu et al., 2017; Zhou et al., 2019). Upon ligand perception, cell surface receptors form a complex with different SERKs (Ma et al., 2016; Wan et al., 2019). In addition, cytoplasmic RLCKs act as phosphorylation substrates of activated receptor-containing complexes and subsequently activate downstream immune responses, such as ROS production and MAPK activation (Cui et al., 2018; Liang and Zhou, 2018). Hence, comparative analysis of signalling pathways mediated by cell surface receptors might offer insight into the signalling pathway mediated by a given RLK or RLP.

So far, various reports have demonstrated that some RLKs and RLPs share the same component to mediate plant immunity. For instance, both RLKs and RLPs form ligandinduced complexes with the regulatory RLK BAK1, and require this RLK for the activation of downstream signalling (Gust and Felix, 2014; Liebrand et al., 2014; Ma et al., 2016; van der Burgh and Joosten, 2019; Wan et al., 2019). In addition, the RLK BIR2 and the protein phosphatases PP2A-A1/C4, which target BAK1 to negatively regulate both FLS2 and EFR signalling (Halter et al., 2014b; Segonzac et al., 2014), also negatively regulate RLP23 signalling (Wan et al., 2018). Moreover, the MAPK cascade MAPKKK3/5-MKK4/5-MPK3/6, or the cascade composed of their respective homologues, is involved in signalling pathways mediated by both RLKs, like FLS2 and EFR (Bi et al., 2018; Meng and Zhang, 2013; Sun et al., 2018), and RLPs such as RLP23 and Cf-4 (Albert et al., 2015; Melech-Bonfil and Sessa, 2010; Stulemeijer et al., 2007; Zhang et al., 2013a).

It is noteworthy that highly homologous components can also be differently used for signal transduction. For instance, the Arabidopsis RLCK PBL13 negatively regulates immune responses mediated by RLKs including FLS2 (Lin et al., 2015), whereas its orthologue NbACIK1 is required for the HR triggered by both Cf-4/Avr4 and Cf-9/Avr9 (Rowland et al., 2005). Another example is the observation that the E3 ubiquitin ligase 


\section{Chapter 1}

PUB22 negatively regulates immune responses mediated by RLKs such as FLS2 (Furlan et al., 2017; Stegmann et al., 2012; Trujillo et al., 2008), whereas its close homologue NbACRE74 positively regulates the HR triggered by both Cf-4/Avr4 and Cf-9/Avr9 (Gilroy et al., 2011; González-Lamothe et al., 2006). Recently, the wellstudied RLCK BIK1, which is a positive regulator of FLS2 signalling (Zhang et al., 2010), was shown to negatively regulate RLP23 signalling (Wan et al., 2018). In addition, the same phosphorylation sites of BIK1 are required for its positive role in FLS2 signalling and for its negative role in RLP23 signalling (Lin et al., 2014; Wan et al., 2018; Zhang et al., 2010). Strikingly, CPK28 and heterotrimeric G-proteins, which antagonistically regulate FLS2 signalling through regulating the accumulation of BIK1 (Monaghan et al., 2014; Wang et al., 2018a), also antagonistically regulate RLP23 signalling, regardless of the opposite role of BIK1 in FLS2 and RLP23 signalling (Wan et al., 2018). So far, the molecular mechanism underlying the opposite role of BIK1 remains unknown.

Although a certain signalling component might employ different molecular mechanisms to regulate plant immunity mediated by different cell surface receptors, the wellcharacterized FLS2 signalling pathway can be used as a model to study signalling pathways mediated by other receptors, such as Cf-4. To date, it is known that Cf-4 requires SOBIR1 and BAK1 to mediate resistance to strains of $C$. fulvum secreting Avr4, and that the Avr4-induced recruitment of BAK1 to the constitutive Cf-4/SOBIR1 complex results in phosphorylation of SOBIR1 and possibly also BAK1 (Liebrand et al., 2013; Postma et al., 2016; van der Burgh et al., 2019). However, components involved in regulating the Avr4-induced formation and subsequent activation of the Cf4/SOBIR1/BAK1 complex are unknown. To improve our understanding of this process, Cf-4/SOBIR1/BAK1-interacting proteins will have to be identified. In addition to conventional methods, such as immunoprecipitation coupled with mass spectrometry analysis or yeast two-hybrid assays, investigating the possible requirement of components, which regulate FLS2/BAK1 complex formation, for Cf-4 signalling offers an alternative way to study the regulation of $\mathrm{Cf}-4$ signalling.

Although several downstream components required for Cf-4 signalling have been identified (Liebrand et al., 2013; Postma et al., 2016; Stergiopoulos and de Wit, 2009), our knowledge of the Cf-4 signalling pathway is relatively limited when compared to what is known about FLS2 signalling. Emerging evidence has revealed that some intracellular effectors from unrelated pathogens can target the same host signalling components to suppress plant immunity, and has led to the notion that such effectors can be used as molecular probes to dissect plant immunity (Lee et al., 2013; Toruno et al., 2016; Win et al., 2012). In agreement with this, Cf-4 signalling can be suppressed 
by the oomycete pathogen Phytophthora infestans RXLR effectors PexRD2 and AVR3a (Gilroy et al., 2011; King et al., 2014). PexRD2 targets NbMAPKKKE to inhibit activation of downstream MPKs, while AVR3a interacts with NbACRE74 to suppress its E3 ligase activity that is required for its positive role in Cf-4 signalling (Gilroy et al., 2011; King et al., 2014). The model bacterial pathogen Pst DC3000 also injects various T3Es into its host cells, and of which some T3Es target BAK1 that is also required for Cf-4 signalling (Büttner, 2016; Couto and Zipfel, 2016). Therefore, investigating the ability of the various Pst DC3000 T3Es, either with or without known targets, to suppress the Cf-4/Avr4-triggered HR offers an alternative way to identify components involved in the Cf-4 signalling pathway.

\section{Cell surface receptors in plant development}

Besides functioning in immunity, RLKs and RLPs also play a role in development (Jamieson et al., 2018; Ma et al., 2016; Wang et al., 2010a; Wu et al., 2016b). So far, some RLKs have been demonstrated to directly bind endogenously secreted ligands to regulate various developmental processes (Hohmann et al., 2017; Ma et al., 2016; Shinohara and Matsubayashi, 2015; Song et al., 2017a). Similar to RLKs involved in immunity, RLKs involved in development also require regulatory RLKs to activate downstream signalling (Hu et al., 2018; Ma et al., 2016). RLPs involved in development have also been reported (Jamieson et al., 2018; Wang et al., 2010a). However, it appears that these RLPs do not function as ligand-binding receptors, but act as regulators to participate in RLK-mediated signalling pathways (Holzwart et al., 2018; Jeong et al., 1999; Kayes and Clark, 1998; Nadeau and Sack, 2002; Wolf et al., 2014; Yang and Sack, 1995).

\section{RLKs in plant development}

The RLK CLAVATA1 (CLV1) recognizes the stem cell-secreted peptide CLV3 to negatively regulate the activity of the shoot apical meristem (SAM) in various plant species, including Arabidopsis (Fig. 4A), rice, tomato and maize (Bommert et al., 2005; Clark et al., 1993; Clark et al., 1995; Clark et al., 1997; Fletcher et al., 1999; Suzaki et al., 2004; Xu et al., 2015). In Arabidopsis, inactivation of CLV3 signalling results in various developmental phenotypes, including an enlarged SAM, more carpels on the siliques and more rosette leaves (Clark et al., 1995; Fletcher et al., 1999). CLV1 directly binds CLV3 and functions as a primary CLV3 receptor in parallel to the RLKs BARELY ANY MERISTEMs (BAMs) and RECEPTOR-LIKE PROTEIN KINASE 2/TOADSTOOL 2 (RPK2/TOAD2; further referred to as RPK2) (Casamitjana-Martinez et al., 2003; DeYoung et al., 2006; Kinoshita et al., 2010; Ogawa et al., 2008; Shinohara and Matsubayashi, 2015; Somssich et al., 2016b). 
CLV3 belongs to the CLV3/ENDOSPERM SURROUNDING REGION (ESR)-related (CLE) family consisting of 32 members in Arabidopsis (Jun et al., 2008). The 12-aminoacid peptide TRACHEARY ELEMENT DIFFERENTIATION INHIBITORY FACTOR (TDIF) corresponds to the identical CLE domain of CLE41 and CLE44, and is directly recognized by the RLK TDIF RECEPTOR (TDR), which is also known as PHLOEM INTERCALATED WITH XYLEM (PXY), to regulate vascular development (Fisher and Turner, 2007; Hirakawa et al., 2008; Ito et al., 2006; Zhang et al., 2016a) (Fig. 4B).

The RLK BRI1 directly recognizes brassinolide (BL), which is the most active form of the brassinosteroids, to regulate growth in Arabidopsis (Hothorn et al., 2011; Li and Chory, 1997; She et al., 2011) (Fig. 4C). BRI1 signalling has also been demonstrated in crop plants, like rice and tomato (Vert et al., 2005). In addition, HAESA (HAE) and its orthologues directly recognize the endogenously secreted peptide INFLORESCENCE DEFICIENT IN ABSCISSION (IDA) to regulate organ abscission (Butenko et al., 2003; Cho et al., 2008; Santiago et al., 2016) (Fig. 4D). Moreover, PHYTOSULFOKINE RECEPTOR1 (PSKR1) directly recognizes the secreted peptide PHYTOSULFOKINE (PSK) to regulate multiple developmental processes (Amano et al., 2007; Matsubayashi, 2014; Matsubayashi et al., 2002; Wang et al., 2015) (Fig. 4E). Furthermore, RLKs of the ERECTA family (ERfs) recognize the secreted peptides EPIDERMAL PATTERNING FACTOR1 (EPF1) and EPF2 to regulate stomatal development (Hara et al., 2007; Hunt and Gray, 2009; Lin et al., 2017; Shpak et al., 2005) (Fig. 4F).

\section{Complex formation between RLK-type receptors and their regulatory RLKs}

Similar to RLKs mediating immunity, RLKs involved in development also require regulatory RLKs to activate downstream signalling (Ma et al., 2016). So far, it has been demonstrated that the SERKs interact with TDR, BRI1, HAE, PSKR1 and the ERfs in a ligand-dependent manner (Fig. 4B-F), which is similar to their association with FLS2 (He et al., 2007; Ma et al., 2016; Meng et al., 2015; Meng et al., 2016; Wang et al., 2015; Zhang et al., 2016b). Moreover, similar to FLS2, TDR, BRI1 and HAE also require the SERKs as co-receptors, and their ligands also function as a molecular glue to stabilize the heterodimerization of the ectodomains of the ligand-bound RLKs and the SERKs (Hohmann et al., 2017; Santiago et al., 2016; Santiago et al., 2013; Song et al., 2017a; Sun et al., 2013a; Zhang et al., 2016b). The ligand-induced heterodimerization of the ectodomains of the PSK-bound PSKR1 and SERKs is structurally similar to those described above, except that the SERKs do not directly interact with PSK (Wang et al., 2015).

The SERKs are paralogues of four RLKs that are referred to as CLV3-INSENSITIVE 
RECEPTOR KINASEs (CIKs) (Hu et al., 2018). Recently, it was reported that the CIKs function as regulatory RLKs for CLV1 (Fig. 4A) and RPK2 in regulating CLV3 signalling (Hu et al., 2018). However, unlike the SERKs forming ligand-induced complexes with RLKs, the ClKs interact with CLV1 and RPK2 in a ligand-independent manner (Hu et al., 2018; Ma et al., 2016). Currently, it remains unknown whether the ClKs also heterodimerize with CLV1 and RPK2 through their ectodomains.

SOBIR1, which is also known as EVERSHED (EVR), is involved in HAE-mediated regulation of organ abscission (Leslie et al., 2010) (Fig. 4D). The exact role of SOBIR1 in the regulation of organ abscission remains elusive, but it has been proposed that SOBIR1 regulates the internalization of HAE to mediate its negative role in organ abscission (Leslie et al., 2010; Liljegren, 2012; Patharkar and Walker, 2018).

\section{RLPs in plant development}

So far, only a few RLPs have been reported to play a role in development (Jamieson et al., 2018; Wang et al., 2010a). One of the most well-known RLPs involved in development is CLV2, which is Arabidopsis RLP10 and participates in CLV3 signalling in the shoot (Fig. 4A), as well as in CLE signalling in the root (Fiers et al., 2005; Jeong et al., 1999; Kayes and Clark, 1998). In addition, functional CLV2 orthologues have also been reported in maize and tomato (Taguchi-Shiobara et al., 2001; Xu et al., 2015). In addition, TOO MANY MOUTHS (TMM), which is also known as Arabidopsis RLP17, is involved in EPF1/2-mediated regulation of stomatal development (Nadeau and Sack, 2002; Yang and Sack, 1995) (Fig. 4F). Furthermore, RLP44 is involved in the regulation of cell wall integrity and vascular development through the activation of BRI1 and PSKR1 signalling in Arabidopsis (Holzwart et al., 2018; Wolf et al., 2014) (Fig. 4C and E).

Although TMM and RLP44 are required for developmental signalling, they do not function as ligand-binding receptors, but rather act as signalling modulators to regulate these developmental processes. For instance, regulation of stomatal development is mediated by the secreted peptides EPF1/2, which trigger the association of ERfs with SERKs (Hara et al., 2007; Hunt and Gray, 2009; Meng et al., 2015; Shpak et al., 2005). TMM and the ERfs form constitutive complexes, which contribute to ERf-mediated recognition of EPF1/2 (Lee et al., 2012; Lin et al., 2017) (Fig. 4F). In addition, TMM also constitutively interacts with the SERKs in a ligand-independent manner (Meng et al., 2015) (Fig. 4F). Furthermore, RLP44 functions as a scaffold to stabilize the association of BAK1 with BRI1 and PSKR1, and regulates BRI1 and PSKR1 signalling to participate in plant development (Holzwart et al., 2018; Wolf et al., 2014) (Fig. 4C and $E$ ). 


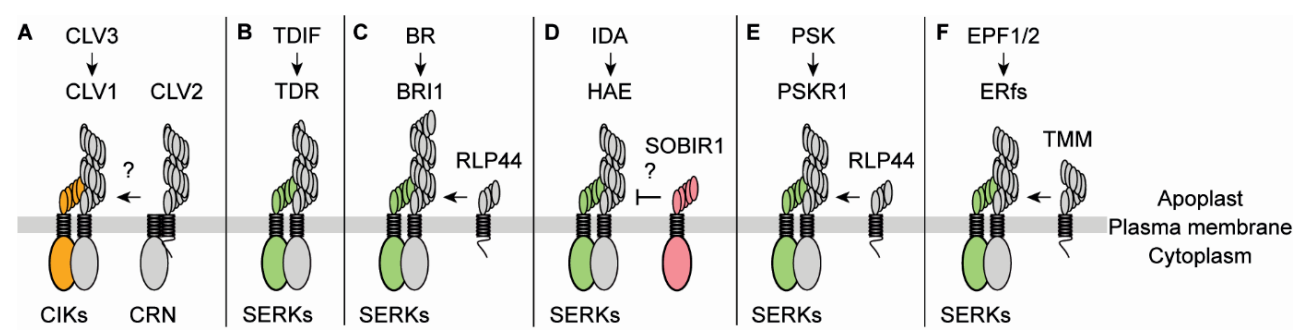

Figure 4. Overview of plasma membrane-associated receptor complexes involved in development in Arabidopsis. (A) The RLK CLV1 directly recognizes CLV3, and constitutively interacts with the CIK regulatory RLKs. The RLP CLV2 and the pseudokinase CRN form a constitutive complex that plays a role in CLV3 signalling. The question mark indicates that the CLV2/CRN complex might participate in the CLV1/CIK complex to regulate CLV3 signalling. (B-D) The RLKs TDR, BRI1 and HAE and the SERK co-receptors form ligand-induced complexes that directly recognize the ligands TDIF, BL and IDA, respectively. Note that RLP44 constitutively interacts with BRI1 and BAK1, and stabilizes the BRI1/BAK1 complex (C). In addition, the RLK SOBIR1 plays a negative role in HAE signalling (D). The question mark indicates that SOBIR1 might regulate the internalization of HAE to mediate its role in HAE signalling. (E) The RLK PSKR1 directly recognizes PSK and forms a ligand-induced complex with the SERK regulatory RLKs. Note that the SERKs do not directly interact with PSK. (F) The RLK receptors ERfs form ligand-induced complexes with the SERKs. Note that the RLP TMM and the ERfs form constitutive complexes, which directly bind EPF1/2. In addition, TMM also constitutively interacts with the SERKs.

\section{The CLV2-dependent CLE signalling in Arabidopsis}

CLV2 constitutively interacts with CORYNE/SUPPRESSOR OF LLP1-2 (CRN/SOL2, further referred to as $\mathrm{CRN}$ ), which lacks an obvious extracellular domain and contains an enzymatically inactive intracellular kinase domain (Bleckmann et al., 2010; Miwa et al., 2008; Müller et al., 2008; Nimchuk et al., 2011; Zhu et al., 2010b) (Fig. 4A). The CLV2/CRN complex is required for perception of CLV3 in the shoot (Jeong et al., 1999; Kayes and Clark, 1998; Miwa et al., 2008; Müller et al., 2008) (Fig. 4A). However, CLV2 does not directly bind CLV3 (Shinohara and Matsubayashi, 2015), and the exact role of the CLV2/CRN complex in perceiving the CLV3 signal remains elusive (Somssich et al., 2016b). Besides playing a role in the shoot, the CLV2/CRN complex is also involved in CLE signalling in the root. Overexpression of various CLE genes, including CLV3, or in vitro application of peptides corresponding to the conserved CLE domain of these CLEs inhibits root elongation (Fiers et al., 2005; Kinoshita et al., 2007). In addition, the CLV2/CRN complex is required for CLE-mediated suppression of root elongation, although the molecular mechanism behind this process is not fully understood (Fiers et al., 2005; Miwa et al., 2008). 
Signalling components downstream of CLV3 receptors in the SAM

Although multiple receptors required for CLV3 perception in the SAM have been identified, only a few downstream signalling components are known (Somssich et al., 2016b). For instance, the protein phosphatase KAPP, which interacts with FLS2 and negatively regulates flg22-triggered immune responses (Gómez-Gómez et al., 2001), targets and dephosphorylates the intracellular kinase domain of CLV1 to negatively regulate CLV3 signalling (Stone et al., 1998; Williams et al., 1997). In addition, the G $\beta$ subunit $A G B 1$ and the Gy subunits $A G G 1 / 2$, which are required for FLS2 signalling (Liang et al., 2016), function downstream of CLV1, RPK2 and CLV2 to regulate CLV3 signalling (Ishida et al., 2014). The requirement of the Ga subunit GPA1 for CLV3 signalling differs in different plant species, as GPA1 is not required for CLV3 signalling in Arabidopsis (Ishida et al., 2014), whereas the maize orthologues of GPA1 and CLV2 form a complex to regulate CLV3 signalling (Bommert et al., 2013). Furthermore, the protein phosphatases POLTERGEIST (POL) and POL-LIKE1 function downstream of CLV3 receptors and maintain the expression of the transcription factor WUSCHEL, which positively regulates the size of the SAM (Laux et al., 1996; Schoof et al., 2000; Song et al., 2006; Yu et al., 2000).

\section{Thesis outline}

So far, it is known that Avr4 triggers the association of the constitutive Cf-4/SOBIR1 complex with BAK1 (Liebrand et al., 2013; Postma et al., 2016). In addition, formation of the Cf-4/SOBIR1/BAK1 complex is proposed to trigger the phosphorylation of the intracellular kinase domains of SOBIR1 and BAK1, which subsequently activate downstream signalling resulting in plant immunity (van der Burgh et al., 2019). However, components involved in regulating the Avr4-induced formation and subsequent activation of the Cf-4/SOBIR1/BAK1 complex remain unknown. In addition, downstream components required for $\mathrm{Cf}-4$ signalling are largely unknown. The work described in this thesis is aimed at gaining more insight into the molecular mechanisms of Cf-4 signalling. Furthermore, the application of the gained knowledge to genetically engineer plant immunity is pursued. Additionally, as SOBIR1, which is required for RLPs mediating immunity, also interacts with the RLP CLV2 involved in development (Liebrand et al., 2013), we investigated the significance of the CLV2/SOBIR1 complex.

In Chapter 2, we set out to study which components are required for controlling the Avr4-induced formation and activation of the Cf-4/SOBIR1/BAK1 complex. Since BIR2 and BIR3 negatively regulate the ligand-induced FLS2/BAK1 complex formation in Arabidopsis (Halter et al., 2014b; Imkampe et al., 2017), we hypothesized that BIR2 and BIR3 might also play a role in Cf-4 signalling in N. benthamiana. To test this hypothesis, we investigated the effect of heterologously expressed BIR2 and BIR3 on 
the HR triggered upon the activation of Cf-4-mediated signalling, and the formation of the Cf-4-containing complex.

Based on the hypothesis that Pst DC3000 T3Es can be used as probes to identify components required for $\mathrm{Cf}-4$ signalling, we selectively studied whether the well-known T3E AvrPto affects Cf-4 signalling (Chapter 3). We studied the effect of AvrPto on the Cf-4/Avr4-triggered HR. Furthermore, we also investigated whether AvrPto interacts with SOBIR1, and tested whether AvrPto affects the Avr4-induced formation of the Cf4/SOBIR1/BAK1 complex.

Studies on cell surface chimeric receptor proteins have revealed that the ectodomain of such chimeric receptors determines ligand specificity, while the transmembrane domain and the cytoplasmic kinase domains determine their output intensity (Boutrot and Zipfel, 2017; De Lorenzo et al., 2011). In Chapter 4, we aimed to obtain a strong MAMP-dependent resistance response in $N$. tabacum, by exploiting the EFR-mediated recognition of the MAMP elf18 and the ability of Cf- 9 to trigger a strong HR (Jones et al., 1994; Zipfel et al., 2006). To this end, we generated the chimera EFR-Cf-9 by replacing the ectodomain of Cf-9 with that of EFR. We further studied whether expression of this chimera in $N$. tabacum resulted in elf18-triggered immunity to bacterial infection. Moreover, we studied whether SOBIR1 and BAK1 are required for the functionality of the chimera EFR-Cf-9.

It is known that SOBIR1 functions as a regulatory RLK for RLPs involved in immunity (Gust and Felix, 2014; Liebrand et al., 2014; van der Burgh and Joosten, 2019; Wan et al., 2019). However, SISOBIR1 also interacts with the RLP SICLV2 (Liebrand et al., 2013), which is involved in S/CLV3 signalling in development (Xu et al., 2015). Currently, the significance of the CLV2/SOBIR1 complex remains unknown. As the roles of both CLV2 and SOBIR1 are conserved across plant species (van der Burgh and Joosten, 2019; Wan et al., 2019), we anticipated that Arabidopsis CLV2 would also interact with SOBIR1, and attempted to investigate the significance of this complex in Arabidopsis. In Chapter 5, we show that Arabidopsis CLV2 indeed also interacts with SOBIR1. In addition, CLV2 also interacts with BAK1. The significance of the interaction of CLV2 with SOBIR1 and BAK1 was determined both in relation to immune responses depending on SOBIR1 and/or BAK1, and in relation to CLV2-dependent CLV3 developmental signalling. Additionally, we attempted to identify downstream components required for CLV3 signalling. As a functional CLV3 signalling pathway requires the G-protein subunits $A G B 1$ and $A G G 1 / 2$, which are also required for FLS2 signalling (Ishida et al., 2014; Liang et al., 2016), we anticipated that other regulators of FLS2 signalling might also play a role in CLV3 signalling. To this end, we examined 
Arabidopsis mutants and transformants displaying impaired FLS2 signalling for their responsiveness to exogenous application of CLV3 peptide in the root.

In Chapter 6, the results presented in this thesis are discussed in a broader context, with the aim to illustrate the regulation of LRR-type cell surface receptor complexes involved in defence and development. 



\section{Chapter 2}

Receptor-like kinases BIR2 and BIR3 interact with SOBIR1 to negatively regulate $\mathrm{Cf}-4$ signalling

Jinbin $\mathrm{Wu}^{1}$, Xiaoyu Hou${ }^{1}$ and Matthieu H.A.J. Joosten ${ }^{1}$

'Laboratory of Phytopathology, Wageningen University \& Research, Droevendaalsesteeg 1, 6708 PB Wageningen, The Netherlands 


\section{Abstract}

The tomato receptor-like protein Cf-4 depends on the receptor-like kinases SUPPRESSOR OF BIR1-1 (SOBIR1) and BRI1-ASSOCIATED KINASE 1 (BAK1) to mediate resistance to strains of the biotrophic fungal pathogen Cladosporium fulvum secreting the effector Avr4. Transient expression of Avr4 in Cf-4-transgenic Nicotiana benthamiana and of Arabidopsis thaliana (At) SOBIR1 in N. benthamiana reconstitutes a signalling pathway similar to the Cf-4 signalling pathway in tomato, and both trigger a typical hypersensitive response (HR). As overexpressed BAK1-INTERACTING KINASE 2 (BIR2) and BIR3 both suppress BAK1-dependent immunity in Arabidopsis, we hypothesized that BIR2 and BIR3 might also suppress Cf-4 signalling in $N$. benthamiana. Here, we show that BIR2 and BIR3 both interact with AtSOBIR1 and suppress the AtSOBIR1-triggered HR in N. benthamiana. In addition, transiently overexpressed BIR2 and BIR3 both inhibit the formation of the constitutive Cf4/SOBIR1 complex and suppress the Avr4-triggered HR in Cf-4-transgenic N. benthamiana, likely through their interaction with SOBIR1. Moreover, AtSOBIR1 phosphorylates BIR2 and BIR3 that both have a stronger interaction with a kinaseinactive mutant of AtSOBIR1 than with wild type AtSOBIR1, suggesting that the phosphorylation of BIR2 and BIR3 reduces their affinity for AtSOBIR1. Taken together, these results indicate that BIR2 and BIR3 interact with SOBIR1 to negatively regulate Cf-4 signalling. Further research is required to determine whether the functional tomato orthologues of BIR2 and BIR3 similarly interact with SOBIR1 to regulate Cf-4-mediated resistance to $C$. fulvum in tomato. 


\section{Introduction}

Cell surface associated pattern-recognition receptors (PRRs) recognize apoplastic signals to initiate plant immunity (Couto and Zipfel, 2016; van der Burgh and Joosten, 2019; Wan et al., 2019). Leucine-rich repeat (LRR) receptor-like kinases (RLKs) and LRR receptor-like proteins (RLPs) are two major types of PRRs (Boutrot and Zipfel, 2017; Li et al., 2016c; Wan et al., 2019). Compared to RLKs, RLPs lack an intracellular kinase domain to trigger downstream signalling upon ligand recognition (Monaghan and Zipfel, 2012).

RLP-mediated defence against infection by pathogens has been demonstrated in various plant species (Boutrot and Zipfel, 2017; van der Burgh and Joosten, 2019; Wan et al., 2019). RLPs and the RLK SUPPRESSOR OF BIR1-1 (SOBIR1) form a constitutive complex, which recruits the RLK BRI1-ASSOCIATED KINASE 1 (BAK1) in a ligand-dependent manner to initiate downstream signalling (Albert et al., 2015; Domazakis et al., 2018; Du et al., 2015; Gust and Felix, 2014; Liebrand et al., 2013; Liebrand et al., 2014; Postma et al., 2016; Wang et al., 2018c; Zhang et al., 2013a). For instance, the tomato (Solanum lycopersicum, SI) RLP Cf-4 recognizes the apoplastic effector Avr4 to mediate resistance to the biotrophic fungal pathogen Cladosporium fulvum (Joosten et al., 1994; Thomas et al., 1997). Cf-4 and SISOBIR1 form a constitutive complex, which recruits S/BAK1 in an Avr4-dependent manner, thereby leading to phosphorylation of SOBIR1 and BAK1, and activation of downstream mitogen-activated protein kinases (MAPKs) (Liebrand et al., 2013; Postma et al., 2016; Stulemeijer et al., 2007; van der Burgh et al., 2019).

Heterologous co-expression of Cf-4 and Avr4 in Nicotiana benthamiana ( $\mathrm{Nb}$ ) activates downstream immune responses eventually leading to a typical hypersensitive response $(\mathrm{HR})$, which is proposed to be similar to that triggered by Cf-4 and Avr4 in tomato (Gabriëls et al., 2006; Liebrand et al., 2012; Liebrand et al., 2013; Postma et al., 2016; Vossen et al., 2010). NbSOBIR1 is required for Cf-4 signalling in $N$. benthamiana and shares a conserved function with SOBIR1 from Arabidopsis thaliana (At) (Liebrand et al., 2013). In addition, transiently overexpressed AtSOBIR1 triggers constitutive immunity that eventually also leads to a typical HR in $N$. benthamiana $(\mathrm{Wu}$ et al., 2018a). Moreover, similar to Avr4-activated Cf-4/SOBIR1 signalling, AtSOBIR1triggered constitutive immunity requires kinase activity of both SOBIR1 and BAK1, and involves the activation of MAPKs (Liebrand et al., 2013; Postma et al., 2016; Stulemeijer et al., 2007; van der Burgh et al., 2019; Wu et al., 2018a), suggesting that constitutively active AtSOBIR1 in $N$. benthamiana represents the Avr4-activated state of NbSOBIR1 and SISOBIR1 in Cf-4 signalling. Therefore, the usage of AtSOBIR1- 
triggered constitutive immunity offers a suitable tool to further dissect the pathway downstream of the activated Cf-4/SOBIR1 complex.

The Arabidopsis BAK1-INTERACTING RECEPTR-LIKE KINASE (BIR) family consists of four members, which all have an ectodomain containing five LRRs and an intracellular domain consisting of a juxta membrane domain and a kinase domain (Blaum et al., 2014; Gao et al., 2009; Halter et al., 2014b; Hohmann et al., 2018; Ma et al., 2017). BIR1 is a kinase-active RLK, whereas BIR2, BIR3 and BIR4 are all pseudokinases (Blaum et al., 2014; Gao et al., 2009; Halter et al., 2014b; Imkampe et al., 2017). Currently, BIR1, BIR2 and BIR3 have been reported to regulate BAK1dependent plant immunity. It has also been demonstrated that BAK1 regulates plant basal defence together with SOBIR1 (Liu et al., 2016), and that BAK1 is also required for the RLK FLAGELLIN-SENSING 2 (FLS2)-mediated defence against infection by the bacterial pathogen Pseudomonas syringae pv. tomato (Pst) DC3000 in Arabidopsis (Chinchilla et al., 2007; Gómez-Gómez and Boller, 2000; Schulze et al., 2010). BIR1 negatively regulates plant basal defence depending on both SOBIR1 and BAK1, but BIR1 does not affect BAK1-dependent FLS2 signalling (Gao et al., 2009; Liu et al., 2016). In contrast, both BIR2 and BIR3 interact with BAK1 to negatively regulate FLS2 signalling (Halter et al., 2014b; Imkampe et al., 2017). So far, it remains unknown how these three BIRs differentially regulate BAK1-dependent plant immunity. As all BIRs share a common mechanism in interacting with BAK1 via their ectodomains, it was proposed that the different roles of the BIRs in regulating plant immunity are caused by the differences in their intracellular domains (Hohmann et al., 2018; Ma et al., 2017).

As both BIR2 and BIR3 suppress BAK1-dependent FLS2 signalling (Halter et al., 2014b; Huang et al., 2017; Imkampe et al., 2017), we hypothesized that transiently overexpressed BIR2 and BIR3 might also suppress Cf-4 signalling. Because AtSOBIR1-triggered constitutive immunity offers a tool to study signalling events occurring downstream of the Cf-4/SOBIR1 complex, we initially studied whether transiently overexpressed BIR2 and BIR3 affect AtSOBIR1-triggered constitutive immunity. Here we show that transiently overexpressed BIR2 and BIR3 both interact with AtSOBIR1 and suppress the AtSOBIR1-triggered HR in N. benthamiana. In addition, BIR2 and BIR3 are phosphorylated by AtSOBIR1. Moreover, transiently overexpressed BIR2 and BIR3 both inhibit the formation of the constitutive Cf4/SOBIR1 complex and suppress the Avr4-triggered HR in Cf-4-transgenic N. benthamiana. Further research is required to determine whether the tomato orthologues of BIR2 and BIR3 similarly interact with SISOBIR1 to regulate Cf-4mediated resistance to $C$. fulvum in tomato. 


\section{Results}

\section{Transiently co-expressed BIR2 and BIR3 suppress AtSOBIR1-triggered constitutive immunity in $\boldsymbol{N}$. benthamiana}

Based on the hypothesis that transiently overexpressed BIR2 and BIR3 might suppress Cf-4 signalling, we initially studied whether BIR2 and BIR3 affect AtSOBIR1triggered constitutive immunity. Therefore, C-terminally eYFP-tagged BIR2 and BIR3, as well as BIR1 and BIR4 (Halter et al., 2014b), were co-expressed with AtSOBIR1eGFP in the presence of the silencing suppressor P19 (Voinnet et al., 2015), which increases overall protein accumulation levels, in $N$. benthamiana. Co-expression of $\beta$ glucuronidase (GUS)-eGFP was used as a negative control. We found that the AtSOBIR1-triggered HR was not affected by GUS-eGFP, BIR1-eYFP and BIR4-eYFP, but was suppressed by eYFP-tagged BIR2 and BIR3 (Fig. 1), indicating that BIR2 and BIR3 are negative regulators of AtSOBIR1-triggered constitutive immunity in $N$. benthamiana.

A

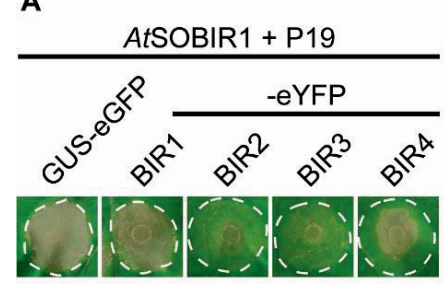

$N$. benthamiana
B

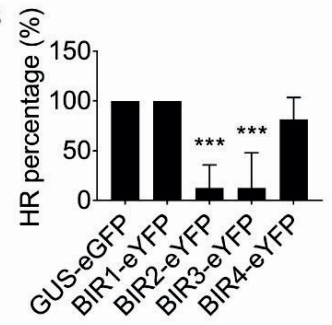

Figure 1. Transiently co-expressed BIR2 and BIR3 suppress AtSOBIR1-triggered constitutive immunity. (A) Transiently co-expressed BIR2 and BIR3 suppress the AtSOBIR1-triggered hypersensitive response (HR) in Nicotiana benthamiana. AtSOBIR1-eGFP and silencing suppressor P19 were co-expressed with GUS-eGFP or C-terminally eYFP-tagged BIRs in leaves of N. benthamiana $(n=8)$. Pictures were taken at 3 days post-infiltration (dpi). The experiment was repeated three times with similar results, and representative results are shown. (B) Quantification of the percentage of the HR as shown in (A). The Student's $t$-test was used for statistical analysis, and asterisks indicate a statistically significant difference $\left.{ }^{* * *} P<0.001\right)$.

\section{BIR2 and BIR3, as well as BIR1 and BIR4, interact with AtSOBIR1}

To study whether BIR2 and BIR3 interact with AtSOBIR1, eYFP-tagged BIR2 and BIR3 were co-expressed with AtSOBIR1-Myc in the presence of P19 in N. benthamiana. BIR1-eYFP and BIR4-eYFP were also included, and GUS-eGFP was used as a negative control. Immunoprecipitation (IP) of eGFP- and eYFP-tagged proteins resulted in the co-purification of AtSOBIR1-Myc with eYFP-tagged BIRs, but not with GUS-eGFP (Fig. 2), indicating that BIR2 and BIR3, as well as BIR1 and BIR4, interact with AtSOBIR1. 


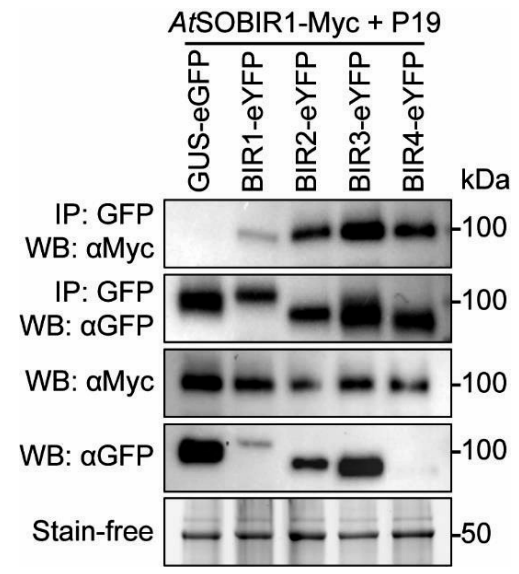

Figure 2. BIR2 and BIR3, as well as BIR1 and BIR4, interact with AtSOBIR1. GUS-eGFP and C-terminally eYFPtagged BIRs were transiently co-expressed with AtSOBIR1Myc in the presence of P19 in N. benthamiana. Proteins were extracted and subjected to immunoprecipitation (IP) by using GFP-affinity beads, followed by western blotting (WB). aGFP antibody was used to detect eGFP- and eYFP-tagged proteins, and aMyc antibody was used to detect AtSOBIR1Myc. Rubisco bands in the stain-free panel indicate equal loading. The experiment was repeated three times with similar results, and representative results are shown.

To study whether kinase activity of AtSOBIR1 plays a role in its interaction with the BIRs, eYFP-tagged BIRs were co-expressed with Myc-tagged AtSOBIR1 or the kinase-inactive mutant AtSOBIR1RD/N, which is also known as AtSOBIR1 $1^{\mathrm{D4} 89 \mathrm{~N}}$ (Liebrand et al., 2013), in the presence of P19 in N. benthamiana. In addition, eYFPtagged BIRs were also co-expressed with AtSOBIR1-Myc in the absence of P19 as controls. IP of either SOBIR1-Myc (Fig. S1) or eYFP-tagged BIRs (Fig. S2) revealed that in the presence of $\mathrm{P} 19$, the BIRs interacted with higher amounts of AtSOBIR1 ${ }^{\mathrm{RD} / \mathrm{N}}$ protein than with the AtSOBIR1 protein. This was more apparent when eYFP-tagged BIRs were immunoprecipitated and the amounts of co-purified AtSOBIR1-Myc and AtSOBIR1 $1^{\mathrm{RD} / \mathrm{N}}-\mathrm{Myc}$ were determined (Fig. S2). In addition, no interaction between eYFP-tagged BIRs and AtSOBIR1-Myc was detected in the absence of P19, which may be caused by overall low protein accumulation levels (Fig. S2). These results indicate that kinase activity of AtSOBIR1 affects its interaction with the BIRs.

\section{BIR2 and BIR3, as well as BIR1 and BIR4, are phosphorylated by AtSOBIR1}

It has been demonstrated that AtSOBIR1 undergoes auto-phosphorylation (van der Burgh et al., 2019). As AtSOBIR1 interacts with BIR2 and BIR3, as well as with BIR1 and BIR4 (Fig. 2), we hypothesized that AtSOBIR1 might be able to phosphorylate the BIRs in planta. Therefore, eYFP-tagged BIRs were co-expressed with Myc-tagged AtSOBIR1, AtSOBIR1 $1^{\mathrm{RD} / \mathrm{N}}$ or GUS, in the presence of $\mathrm{P} 19$ in $N$. benthamiana.

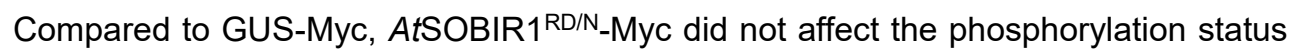
of eYFP-tagged BIRs, whereas AtSOBIR1-Myc phosphorylated all eYFP-tagged BIRs (Fig. 3). These observations indicate that AtSOBIR1 indeed phosphorylates the BIRs in planta. As high accumulation levels of AtSOBIR1 lead to auto-phosphorylation of AtSOBIR1 in N. benthamiana (van der Burgh et al., 2019), eYFP-tagged BIRs were 
also co-expressed with Myc-tagged AtSOBIR1 in the absence of P19, in order to study whether the phosphorylation levels of the BIRs positively correlate with the phosphorylation status of AtSOBIR1. Co-expression with AtSOBIR1-Myc in the absence of P19 also resulted in phosphorylation of all eYFP-tagged BIRs, but to a lesser extent when compared to co-expression with AtSOBIR1-Myc in the presence of P19 (Fig. 3), indicating that P19 promotes the auto-phosphorylation of AtSOBIR1, which in turn leads to higher phosphorylation levels of all BIRs.

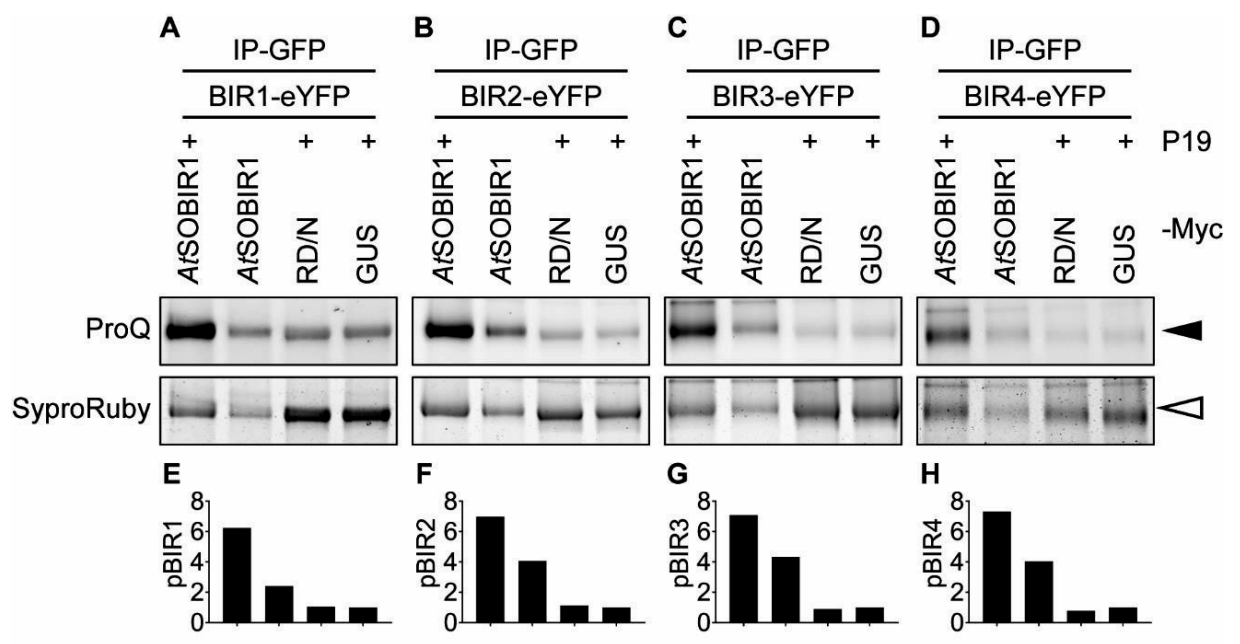

Figure 3. The phosphorylation levels of BIR2 and BIR3, as well as BIR1 and BIR4, depend on the phosphorylation status of co-expressed AtSOBIR1. (A-D) AtSOBIR1 depends on its kinase activity to phosphorylate BIRs, and P19 promotes AtSOBIR1 to phosphorylate all BIRs. C-terminally eYFPtagged BIRs were transiently co-expressed with AtSOBIR1-Myc in the presence or absence of P19, or

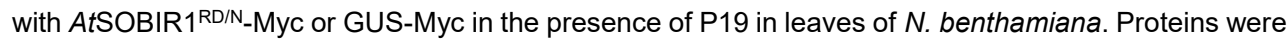
extracted and subjected to IP by using GFP-affinity beads. Immunoprecipitated BIRs were subjected to Pro-Q and Sypro Ruby staining. The black arrowhead indicates the phosphorylated BIR proteins, and the white arrowhead indicates the immunoprecipitated BIR proteins. The experiment was repeated three times with similar results and representative results are shown. (E-H) Quantification of the relative phosphorylation levels of BIRs as shown in (A-D). The phosphorylation level of all BIRs that were coexpressed with GUS was set to 1.

Transiently co-expressed BIR2 and BIR3, as well as BIR4, suppress Cf-4/SOBIR1 signalling in Cf-4-transgenic $\boldsymbol{N}$. benthamiana

The downstream signalling pathway triggered by the overexpressed constitutively active AtSOBIR1 is proposed to be similar to that triggered by the Avr4-activated Cf4/SOBIR1 complex (Liebrand et al., 2013; Postma et al., 2016; Stulemeijer et al., 2007; 
van der Burgh et al., 2019; Wu et al., 2018a). As BIR2 and BIR3 both interact with AtSOBIR1 (Fig. 2) and suppress AtSOBIR1-triggered constitutive immunity (Fig. 1), we studied whether BIR2 and BIR3 also suppress the Avr4-triggered HR in Cf-4transgenic $N$. benthamiana. Therefore, Avr4 was co-expressed with GUS-eGFP, BIR2eYFP and BIR3-eYFP in Cf-4-transgenic N. benthamiana. We found that the Avr4triggered HR was not affected by GUS-eGFP but was suppressed by eYFP-tagged BIR2 and BIR3 (Fig. 4). BIR1-eYFP and BIR4-eYFP were also included. We found that the HR was not affected by BIR1-eYFP but was suppressed by BIR4-eYFP (Fig. 4). Taken together, these results indicate that BIR2 and BIR3, as well as BIR4, suppress Cf-4/SOBIR1 signalling in Cf-4-transgenic N. benthamiana.

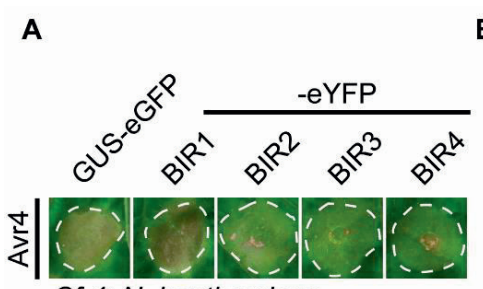

Cf-4: N. benthamiana

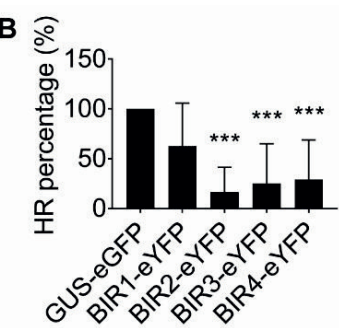

Figure 4. Transiently co-expressed BIR2, BIR3 and BIR4 suppress Cf-4/SOBIR1 signalling. (A) Transiently co-expressed BIR2, BIR3 and BIR4 suppress the Avr4-triggered HR in Cf-4-transgenic N. benthamiana. Avr4 was transiently co-expressed with GUS-eGFP or eYFP-tagged BIRs in leaves of Cf4-transgenic $N$. benthamiana $(n=12)$. Pictures were taken at $3 \mathrm{dpi}$. The experiment was repeated three times with similar results, and representative results are shown. (B) Quantification of the percentage of the HR as shown in (A). The Student's $t$-test was used for statistical analysis, and asterisks indicate a statistically significant difference $\left({ }^{* * *} P<0.001\right)$.

\section{Transiently co-expressed BIR2 and BIR3 inhibit the formation of the constitutive} Cf-4/SOBIR1 complex

As BIR2 and BIR3 interact with AtSOBIR1 (Fig. 2) and suppress the Avr4-activated Cf4/SOBIR1 signalling (Fig. 4), we studied whether overexpression of BIR2 and BIR3 affects the formation of the constitutive Cf-4/SOBIR1 complex. Therefore, GUS-eGFP, BIR2-eYFP and BIR3-eYFP were co-expressed with Cf-4-Myc in N. benthamiana. Subsequently, the interaction between Cf-4-Myc and endogenous NbSOBIR1 was monitored. Compared to GUS-eGFP, eYFP-tagged BIR2 and BIR3 both suppressed the interaction between Cf-4-Myc and endogenous NbSOBIR1 (Fig. 5), indicating that overexpressed BIR2 and BIR3 inhibit the formation of the constitutive Cf-4/SOBIR1 complex. No interaction between Cf-4-Myc and eYFP-tagged BIR2 or BIR3 was detected (Fig. 5). In addition, no interaction between NbSOBIR1 and eYFP-tagged 
BIR2 or BIR3 was detected either (Fig. 5). The latter observation is reminiscent of a lack of detectable interaction between the BIRs and AtSOBIR1 in the absence of P19 (Fig. S2), suggesting that the amount of endogenous NbSOBIR1 protein interacting with the eYFP-tagged BIR2 and BIR3 proteins is below the threshold of detection. As overexpressed BIR2 and BIR3 both interact with AtSOBIR1 in the presence of P19 (Fig. 2), it is likely that both BIR2 and BIR3 target endogenous NbSOBIR1 to inhibit the formation of the constitutive Cf-4/SOBIR1 complex.

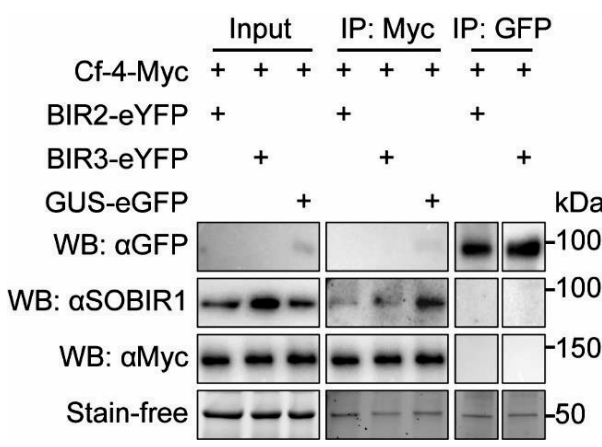

Figure 5. Transiently co-expressed BIR2 and BIR3 inhibit the formation of the constitutive Cf-4SOBIR1 complex. Cf-4-Myc was transiently co-expressed with BIR2-eYFP, BIR3-eYFP or GUS-eGFP in leaves of $N$. benthamiana. Proteins were extracted and subjected to IP by using Myc- or GFP-affinity beads, followed by WB. aMyc was used to detect Cf-4-Myc, aSOBIR1 was used to detect endogenous $\mathrm{NbSOBIR} 1$ and aGFP was used to detect eGFP- and eYFP-tagged proteins. Note that, when compared to GUS-eGFP, eYFP-tagged BIR2 and BIR3 inhibit the interaction between Cf-4-Myc and endogenous NbSOBIR1 (IP: Myc; WB: aSOBIR1). The experiment was repeated twice with similar results, and representative results are shown.

BIR2 and BIR3 negatively regulate RLP-mediated immune responses in Arabidopsis

RLP-mediated defence has also been reported in Arabidopsis (Albert et al., 2015; Jehle et al., 2013a; Jehle et al., 2013b; Zhang et al., 2014; Zhang et al., 2013a). For instance, RLP42 recognizes Botrytis cinerea ENDOPOLYGALACTURONASE 3 (BcPG3) to mediate a SOBIR1-dependent development of macroscopically visible necrotic symptoms in Arabidopsis accession Columbia-0 (Col-0) (Zhang et al., 2014). In addition, RLP23 mediates defence against infection by pathogens producing NECROSIS- AND ETHYLENE-INDUCING PROTEIN 1 (NEP1)-like proteins (NLPs), and recognition of the conserved immunogenic peptide NLP24 of NLPs by RLP23 triggers a SOBIR1-dependent production of reactive oxygen species (ROS) in Col-0 (Albert et al., 2015; Böhm et al., 2014b; Oome et al., 2014). 

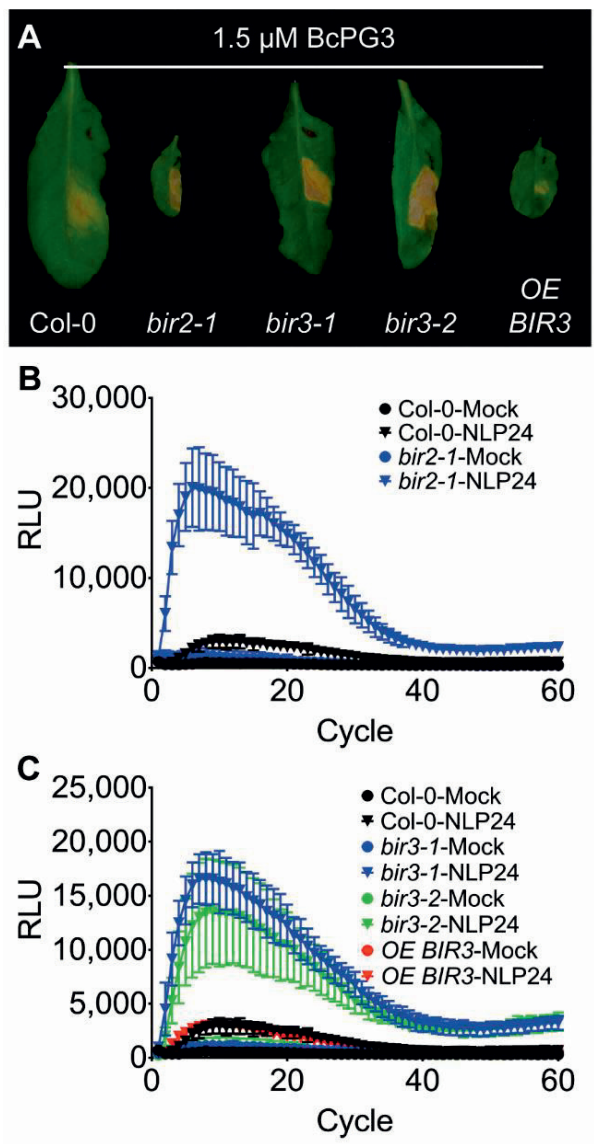

Figure 6. BIR2 and BIR3 negatively regulate RLPmediated immune responses in Arabidopsis thaliana. (A) BIR2 and BIR3 negatively regulate the development of RLP42/BcPG3-triggered necrotic symptoms. Arabidopsis leaves were infiltrated with 1.5 $\mu \mathrm{M} \mathrm{BcPG3}(n=8)$, and pictures were taken at 5 dpi. Note that BcPG3-triggered necrotic symptoms are stronger in mutants of bir2 and bir3, but are suppressed in transgenic lines overexpressing $B I R 3$ (OE BIR3). (B, C) BIR2 (B) and BIR3 (C) negatively regulate the RLP23/NLP24-triggered ROS burst. Arabidopsis leaf discs were treated with either Milli-Q water as a control (Mock) or $1 \mu$ M NLP24 $(n=8)$. Note that the NLP24-triggered ROS burst is strongly enhanced in mutants of bir2 and bir3, but is not affected in transgenic lines overexpressing BIR3. These experiments were repeated three times with similar results, and representative results are shown.

As BIR2 and BIR3 interact with AtSOBIR1 (Fig. 2), and negatively regulate SOBIR1dependent $\mathrm{Cf}-4$ signalling in N. benthamiana (Fig. 4), we studied whether BIR2 and BIR3 also affect the SOBIR1-dependent immune responses mediated by RLP42 and RLP23 in Arabidopsis. Therefore, Arabidopsis mutants bir2-1, bir3-1 and bir3-2, and transgenic lines overexpressing BIR3 (Halter et al., 2014b; Imkampe et al., 2017), were tested for their responsiveness to BcPG3 and NLP24. Compared to Col-0, BcPG3triggered necrotic symptoms appeared earlier and were stronger in bir2-1, bir3-1 and bir3-2 mutants, but were suppressed in transgenic plants overexpressing BIR3 (Fig. 6A). In addition, the NLP24-triggered ROS burst was strongly enhanced in bir2-1, bir31 and bir3-2 mutants, but was not affected in transgenic plants overexpressing BIR3 (Fig. 6B and 6C). These observations indicate that BIR2 and BIR3 negatively regulate immune responses mediated by both RLP42 and RLP23. 
BIR4 also interacts with AtSOBIR1 (Fig. 2). Although BIR4 does not affect the AtSOBIR1-triggered HR (Fig. 1), it does suppress the Avr4-triggered HR in Cf-4transgenic N. benthamiana (Fig. 4). To study whether BIR4 regulates the SOBIR1dependent immune responses mediated by RLP42 and RLP23 in Arabidopsis, bir4-2 and bir4-3 mutants (Wierzba, 2013) were tested for their responsiveness to BcPG3 and NLP24. Compared to Col-0, the development of BcPG3-triggered necrotic symptoms was suppressed in bir4-2 and bir4-3 mutants (Fig. S3A). In addition, the NLP24-triggered ROS burst was suppressed in bir4-2 and bir4-3 mutants (Fig. S3B). These observations indicate that BIR4 is a positive regulator of immune responses mediated by both RLP42 and RLP23.

BIR1 also interacts with AtSOBIR1 (Fig. 2). However, as bir1-1 mutants display a lethal phenotype (Gao et al., 2009), bir1-1 mutants could not be tested for their responses to BcPG3 and NLP24.

Taken together, both BIR2 and BIR3 negatively regulate RLP-mediated immune responses in Arabidopsis, whereas BIR4 appears to positively regulate these responses.

\section{Discussion}

Heterologous expression studies in $\boldsymbol{N}$. benthamiana plants facilitate studies on Cf-4 signalling

Although Cf-4 originates from tomato, heterologously expressed Cf-4 in N. benthamiana mediates a signalling pathway that is proposed to be similar to the Cf-4 signalling pathway in tomato plants (Gabriëls et al., 2006; Liebrand et al., 2012; Liebrand et al., 2013; Postma et al., 2016; Vossen et al., 2010). Moreover, transient overexpression of AtSOBIR1 in N. benthamiana triggers a constitutive immunity that appears to reflect signalling triggered by the Avr4-activated Cf-4/SOBIR1 complex (Liebrand et al., 2013; Postma et al., 2016; van der Burgh et al., 2019; Wu et al., 2018a).

In this study, we found that transiently co-expressed BIR2 and BIR3 both interact with AtSOBIR1 and suppress AtSOBIR1-triggered constitutive immunity in N. benthamiana (Fig. 1 and 2). In addition, transiently co-expressed BIR2 and BIR3 both inhibit the formation of the constitutive Cf-4/SOBIR1 complex and suppress the Cf-4/Avr4triggered HR (Fig. 4 and 5). Although it remains unknown whether the tomato functional orthologues of BIR2 and BIR3 also interact with SISOBIR1 to regulate Cf-4-mediated resistance to $C$. fulvum in tomato plants, the strategy of using heterologous expression studies offers an alternative way to study the role of tomato orthologues of BIR2 and 
BIR3 in Cf-4 signalling. However, as BIR4 does not affect the AtSOBIR1-triggered HR but does suppress the Cf-4/Avr4-triggered HR (Fig. 1 and 4), it is worth noting that there are subtle differences between the signalling pathways triggered by AtSOBIR1 and Cf-4/Avr4. Therefore, the role of a given regulator needs to be carefully checked by, for example, studying whether its orthologues indeed regulate Cf-4-mediated resistance to C. fulvum in tomato plants.

\section{The dynamic interaction between AtSOBIR1 and the BIRs might depend on BAK1}

Similar to the Avr4-activated Cf-4/SOBIR1 complex, AtSOBIR1, but not the kinaseinactive mutant $A t S O B I R 1^{\mathrm{RD} / \mathrm{N}}$, activates downstream signalling in a manner requiring kinase activity of both SOBIR1 and BAK1, and involving the activation of downstream MAPKs (Liebrand et al., 2013; Postma et al., 2016; Stulemeijer et al., 2007; van der Burgh et al., 2019; Wu et al., 2018a). These observations suggest that overexpression of AtSOBIR1 $1^{\mathrm{RD} / \mathrm{N}}$ represents the resting state, while overexpression of AtSOBIR1 represents the activated state of Cf-4/SOBIR1 signalling.

BIRs are phosphorylated by AtSOBIR1, but not by AtSOBIR1RD/N (Fig. 3), indicating that SOBIR1 phosphorylates the associated BIRs when SOBIR1 is activated. In addition, when compared to AtSOBIR1, higher amounts of AtSOBIR1 ${ }^{\mathrm{RD} / \mathrm{N}}$ interact with the BIRs (Fig. S1 and S2), suggesting that the BIRs dissociate from SOBIR1 when the BIRs are phosphorylated. Although the molecular mechanisms behind the phosphorylation of the BIRs and their dissociation from SOBIR1 remain unknown, it is possible that the BIRs interact with SOBIR1 in the resting state, while in the activated state SOBIR1 phosphorylates the BIRs, leading to their dissociation from SOBIR1.

BAK1 might be involved in the AtSOBIR1-mediated phosphorylation of the BIRs in both an indirect and a direct way. In this study, we found that phosphorylation of the BIRs fully depends on the kinase activity of AtSOBIR1 (Fig. 3). As BAK1 interacts with AtSOBIR1 and is required for full activation of AtSOBIR1 that is possibly caused by trans-phosphorylation events occurring between these two RLKs (van der Burgh et al., 2019), it is likely that upon its association with AtSOBIR1, BAK1 phosphorylates AtSOBIR1, which subsequently phosphorylates the BIRs. In addition, BAK1 might also directly phosphorylate BIR2, BIR3 and BIR4, as it has been reported that BAK1 phosphorylates BIR2, BIR3 and BIR4 in vitro (Halter et al., 2014b; Imkampe et al., 2017). It remains unknown whether BAK1 also directly phosphorylates BIR1. However, as the kinase domain of BAK1 does not interact with that of BIR1 (Halter et al., 2014b), it is likely that this direct phosphorylation does not take place. 


\section{The cytosolic domain of BIRs might determine their specific roles in immunity}

The role of BIR4 in immunity has not been reported yet, whereas BIR1, BIR2 and BIR3 have been demonstrated to differentially regulate BAK1-dependent plant immunity (Gao et al., 2009; Halter et al., 2014b; Imkampe et al., 2017; Liu et al., 2016). As all BIRs interact with BAK1 through their ectodomains in a similar manner, the different roles of BIR1, BIR2 and BIR3 in regulating immunity are supposed to be caused by their intracellular domains (Blaum et al., 2014; Hohmann et al., 2018; Ma et al., 2017). The cytosolic domain of all BIRs consists of a juxta membrane domain and a kinase domain (Blaum et al., 2014). Therefore, both their juxta membrane domains and kinase domains might determine the functional specificity of the BIRs. For instance, the characteristics of their kinase domains might determine the role of the BIRs. When compared to that of BIR2 and BIR3, the kinase domain of BIR1 possesses enzymatic activity and does not interact with the kinase domain of BAK1 (Blaum et al., 2014; Gao et al., 2009; Halter et al., 2014b; Imkampe et al., 2017; Liu et al., 2016), indicating that the intrinsic kinase activity of BIR1 might determine its specific role in regulating immunity. Alternatively, the juxta membrane domain might also determine their roles in regulating immunity. For example, it has been reported that the juxta membrane domain of BIR2 is intensively phosphorylated by BAK1, and the phosphorylated juxta membrane domain is supposed to interact with yet unknown components that might specify the role of BIR2 (Blaum et al., 2014). However, it is also possible that the entire cytosolic domain determines the specific roles of the BIRs.

In this study, we found that the BIRs all interact with AtSOBIR1 (Fig. 2), and are all phosphorylated by AtSOBIR1 (Fig. 3). However, the BIRs differentially regulate both the AtSOBIR1-triggered $\mathrm{HR}$ and the Avr4-triggered HR (Fig. 1 and 4). These observations are reminiscent of the different roles of the BIRs in BAK1-dependent plant immunity (Gao et al., 2009; Halter et al., 2014b; Imkampe et al., 2017; Liu et al., 2016), indicating that the different roles of the BIRs in regulating SOBIR1-dependent immune signalling might also depend on their different intracellular domains. To further study which domain might determine the role of a given BIR member, the kinase domain, the juxta membrane domain or the entire cytosolic domain of this BIR member should be replaced by the corresponding domain from another BIR member, followed by testing the role of the recombinant BIR in regulating SOBIR1-dependent immune signalling.

We unexpectedly found that BIR4 differentially regulates the HR triggered by constitutively active AtSOBIR1 and by the Avr4-activated Cf-4/SOBIR1 complex (Fig. 1 and 4), and even functions as a positive regulator of RLP-mediated immune responses in Arabidopsis (Fig. S3). The molecular mechanism behind the different roles of BIR4 in regulating these immune responses in different plant species is 
unknown. However, as in $N$. benthamiana the Avr4-triggered HR depends on the activity of endogenous NbSOBIR1, while both the AtSOBIR1-triggered HR and RLPmediated immune responses in Arabidopsis depend on the intrinsic activity of AtSOBIR1, it is possible that the differential roles of BIR4 might be related to unknown effects of the protein on activity of NbSOBIR1 and AtSOBIR1.

\section{BIR2 and BIR3 interact with AtSOBIR1, and maybe BAK1, to suppress AtSOBIR1- triggered constitutive immunity}

In this study, we found that BIR2 and BIR3 interact with AtSOBIR1 (Fig. 2), and suppress AtSOBIR1-triggered constitutive immunity in $N$. benthamiana (Fig. 1). However, the molecular mechanism behind BIR2- and BIR3-mediated suppression of AtSOBIR1-triggered constitutive immunity remains unknown. As AtSOBIR1 forms homodimers and this homodimerization is supposed to initiate the autophosphorylation of AtSOBIR1 that leads to constitutive immunity (van der Burgh et al., 2019), further research is required to study whether BIR2 and BIR3 affect homodimerization and/or auto-phosphorylation of AtSOBIR1.

It has been reported that BAK1 interacts with AtSOBIR1, and is required for the phosphorylation of AtSOBIR1 and AtSOBIR1-triggered constitutive immunity (van der Burgh et al., 2019). As BIR2 and BIR3 also interact with BAK1 (Halter et al., 2014b; Imkampe et al., 2017), it is likely that BIR2 and BIR3 also interact with BAK1 to inhibit the phosphorylation of AtSOBIR1, thereby suppressing AtSOBIR1-triggered constitutive immunity. Further research is required to study whether BIR2 and BIR3 affect the interaction and/or phosphorylation events between SOBIR1 and BAK1.

\section{BIR2 and BIR3 interact with SOBIR1, and maybe BAK1, to negatively regulate} RLP-mediated immune signalling

In this study, we found that co-expressed BIR2 and BIR3 inhibit the constitutive interaction between Cf-4 and endogenous NbSOBIR1 and suppress the Cf-4/Avr4triggered HR (Fig. 4 and 5). Although no interaction between endogenous NbSOBIR1 and co-expressed BIR2 or BIR3 could be shown (Fig. 5), BIR2 and BIR3 do interact with AtSOBIR1 (Fig. 2). Therefore, it is likely that BIR2 and BIR3 target endogenous $\mathrm{NbSOBIR} 1$ to inhibit its interaction with Cf-4, thereby suppressing the Avr4-triggered HR in Cf-4-transgenic N. benthamiana. However, this hypothesis needs to be carefully checked by studying whether the constitutive Cf-4/SOBIR1 complex is also affected by BIR1 and BIR4, as both BIR1 and BIR4 also interact with AtSOBIR1, but differentially regulate the Cf-4/Avr4-triggered HR (Fig. 4). Nevertheless, it is clear that BIR2 and BIR3 interact with SOBIR1, and negatively regulate both Cf-4 signalling in $N$. benthamiana and RLP-mediated immune signalling in Arabidopsis (Fig. 2, 4 and 6). 
These observations together indicate that BIR2 and BIR3 interact with SOBIR1 to negatively regulate RLP-mediated immune signalling.

In addition to their interaction with SOBIR1, BIR2 and BIR3 might also interact with BAK1 to negatively regulate RLP-mediated immune signalling. It has been demonstrated that BAK1 interacts with Cf-4 and RLP23 in a ligand-dependent manner, and that BAK1 is required for immunity mediated by these two RLPs (Albert et al., 2015; Postma et al., 2016). In addition, BAK1 and its paralogue BAK1-LIKE 1 (BKK1) are redundantly required for the development of $B c P G 3$-triggered necrotic symptoms (see results in Chapter 5). Although an interaction between the RLP42/SOBIR1 complex and BAK1 has not been reported yet, BAK1 might also interact with RLP42 in a liganddependent manner, as RLP42 has the conserved features of RLPs (Gust and Felix, 2014; Liebrand et al., 2014). In this scenario, as BAK1 is recruited to both FLS2 and RLPs in a ligand-dependent manner (Albert et al., 2015; Chinchilla et al., 2007; Domazakis et al., 2018; Postma et al., 2016; Schulze et al., 2010; Wang et al., 2018c; Zhang et al., 2013a), and BIR2 and BIR3 interact with BAK1 to inhibit the ligandinduced formation of the FLS2/BAK1 complex (Halter et al., 2014b; Imkampe et al., 2017), it is likely that BIR2 and BIR3 also interact with BAK1 to inhibit the ligandinduced recruitment of BAK1 to RLPs, thereby suppressing RLP-mediated immune signalling. Moreover, as BAK1 recruitment to the RLP/SOBIR1 complex is proposed to initiate trans-phosphorylation events between SOBIR1 and BAK1, leading to full activation of the RLP/SOBIR1/BAK1 complex (Postma et al., 2016; van der Burgh et al., 2019), it is likely that BIR2 and BIR3 also interact with BAK1 to inhibit the phosphorylation of both SOBIR1 and BAK1, thereby suppressing the activation of downstream signalling.

\section{Conclusion}

Taken together, we propose a model to illustrate the role of BIR2 and BIR3 in Cf-4 signalling. Cf-4 constitutively interacts with SOBIR1, and BAK1 is recruited to the Cf4/SOBIR1 complex upon perception of Avr4 by Cf-4, resulting in the initiation of possible trans-phosphorylation events between SOBIR1 and BAK1 (Liebrand et al., 2013; Postma et al., 2016; van der Burgh et al., 2019). Activation of the Cf4/SOBIR1/BAK1 complex subsequently triggers downstream signalling eventually leading to immunity. In the resting state (Fig. 7, left panel), both BIR2 and BIR3 constitutively interact with SOBIR1. In addition, overexpressed BIR2 and BIR3 even inhibit the formation of the constitutive Cf-4/SOBIR1 complex to suppress Cf-4 signalling. In the activated state (Fig. 7, right panel), SOBIR1 phosphorylates BIR2 and BIR3, leading to their dissociation from SOBIR1. In this proposed model, BIR2 and 
BIR3 might also interact with BAK1 to regulate $\mathrm{Cf}-4$ signalling. For instance, BIR2 and BIR3 interact with BAK1 to sequester it from the Cf-4/SOBIR1 complex, thereby avoiding auto-activation of the Cf-4/SOBIR1/BAK1 complex (Fig. 7, left panel). In addition, BAK1 might also phosphorylate BIR2 and BIR3, leading to their dissociation from BAK1 (Fig. 7, right panel). Moreover, as both BIR2 and BIR3 also negatively regulate SOBIR1- and BAK1-dependent immune responses mediated by RLP42 and RLP23 in Arabidopsis, the proposed model possibly also applies to RLP signalling in general.

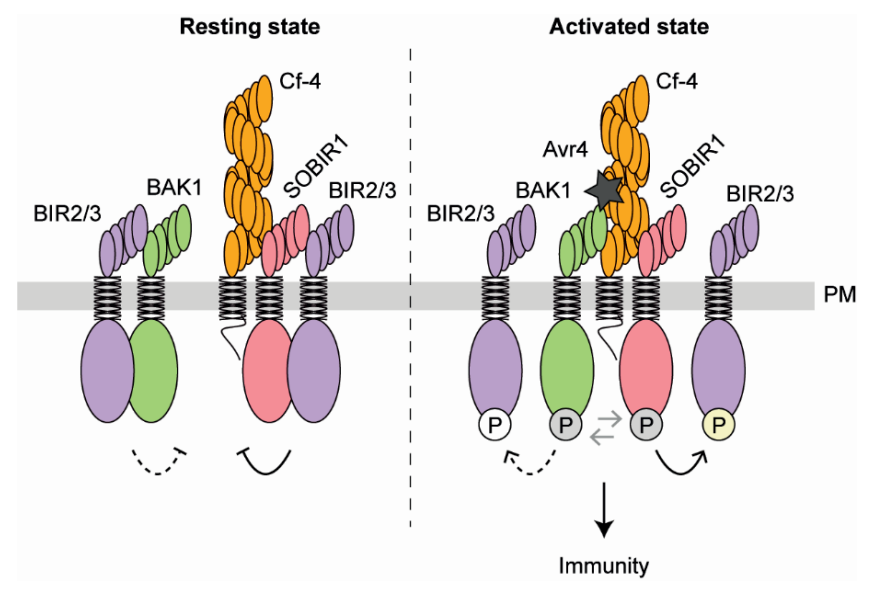

Figure 7. A model illustrating the role of BIR2 and BIR3 in Cf-4 signalling. Cf-4 constitutively interacts with SOBIR1, and BAK1 is recruited to the Cf-4/SOBIR1 complex upon perception of Avr4 by Cf-4, resulting in the initiation of possible trans-phosphorylation events between SOBIR1 and BAK1. Activation of the Cf-4/SOBIR1/BAK1 complex subsequently triggers downstream signalling eventually leading to immunity. In the resting state (left panel), BIR2 and BIR3 interact with SOBIR1. In addition, overexpressed BIR2 and BIR3 even inhibit the formation of the constitutive Cf-4/SOBIR1 complex. In the activated state (right panel), SOBIR1 phosphorylates BIR2 and BIR3, leading to their dissociation from SOBIR1. In addition, BIR2 and BIR3 might also interact with BAK1 to regulate Cf-4 signalling. Black lines and the light-yellow circle represent results from this study, grey arrows and grey circles represent published data, and black dashed lines and the white circle represent a hypothesis based on published data (see text for further details). P, phosphorylation; PM, plasma membrane.

\section{Materials and Methods}

\section{Plant materials and growth conditions}

Arabidopsis thaliana $(A t)$ plants in the Columbia-0 (Col-0) background were used in this study. T-DNA insertion lines bir2-1 (GK-793F12), bir3-1 (Salk_132078) and bir3-2 (Salk_116632), and the transgenic line OE BIR3 (35S::BIR3-FLAG) were described in published reports (Halter et al., 2014b; Imkampe et al., 2017). T-DNA insertion lines 
bir4-2 (CSHL_ET9428) and bir4-3 (CSHL_ET11166) were described in a PhD thesis (Wierzba, 2013). Arabidopsis plants were grown in soil in a climate chamber under $12 \mathrm{~h}$ of light at $21^{\circ} \mathrm{C}$, and $12 \mathrm{~h}$ of darkness at $19^{\circ} \mathrm{C}$, with a relative humidity of $70 \%$.

Wild type (WT) and Cf-4-transgenic Nicotiana benthamiana ( $N b$ ) plants (Gabriëls et al., 2006) were grown in soil in a climate chamber under $16 \mathrm{~h}$ of light at $25^{\circ} \mathrm{C}$ and $8 \mathrm{~h}$ of darkness at $21^{\circ} \mathrm{C}$, with a relative humidity of $75 \%$.

\section{Binary vectors for Agrobacterium-mediated transformation}

Binary vectors pBIN-KS-35S::AtSOBIR1-eGFP, pGWB20-35S::AtSOBIR1-Myc, pGWB20-35S::AtSOBIR1 1089N-Myc, pGWB20-35S::Cf-4-Myc, pBIN-KS-35S::GUSeGFP, pMOG800-35S::Avr4 and pBIN61-35S::P19 have been described previously (Liebrand et al., 2013; van der Hoorn et al., 2000; Voinnet et al., 2015). The fragment of GUS in pENTR/D-TOPO-GUS (Liebrand et al., 2013) was transferred into pGWB20 to generate pGWB20-35S::GUS-Myc. Binary vectors pB7YWG2-35S::BIR1-eYFP, pB7YWG2-35S::BIR2-eYFP, pB7YWG2-35S::BIR3-eYFP and pB7YWG2-35S::BIR4eYFP were described in published reporters (Halter et al., 2014b). Binary vectors were transformed to Agrobacterium tumefaciens strain C58C1. Infiltration of $A$. tumefaciens suspensions (agro-infiltrations) into leaves was performed as described previously (Wu et al., 2018a). Suspension of transformed cells expressing Avr4 was infiltrated at a final $\mathrm{OD}_{600}$ of 0.03 , and the remaining cells were infiltrated at a final $\mathrm{OD}_{600}$ of 1.

\section{Hypersensitive response (HR) scoring}

Fully expanded leaves of four- to six-week-old WT and Cf-4-transgenic N. benthamiana plants were used for agro-infiltrations. The HR percentage was visually scored by determining the cell death intensity, ranging from $0,25,50,75$ to $100 \%$, and the means of the HR percentages are indicated. The Student's $t$-test was used for statistical analysis, and asterisks indicate a statistically significant difference $\left({ }^{\star * *} P<0.001\right)$.

\section{Co-immunoprecipitation (co-IP) assays}

Co-IPs were performed as described previously (Liebrand et al., 2012). aGFP-HRP (Miltenyi Biotec $\mathrm{GmbH}$ ) was used to detect eGFP- or eYFP-tagged proteins. aMyc (Santa Cruz Biotechnology) and aMouse-HRP (GE Healthcare) were used to detect Myc-tagged proteins. aSOBIR1 (Agrisera) and aRabbit-HRP (Agrisera) were used to detect NbSOBIR1. Band intensities in the IP and co-IP panels were measured by ChemiDoc (Bio-Rad), and the ratio of the co-IP signal to the IP signal was determined. The ratio of the indicated control group was set to 1 , and the ratio of treatment groups was normalized to the control group, providing a number indicating the relative interaction level. A number above 1 indicates a higher interaction levels when 
compared with the control group.

\section{Protein phosphorylation assays}

The protein phosphorylation assay was performed as described previously (van der Burgh et al., 2019). Band intensities were measured by ChemiDoc, and the ratio of Pro-Q (Invitrogen) signal to SYPRO Ruby (Invitrogen) signal was determined. The ratio of the control group was set to 1 , and the ratio of treatment groups was normalized to the ratio of the control group, providing a number indicating the relative protein phosphorylation level. A number above 1 indicates a higher phosphorylation levels when compared with the control group.

\section{BcPG3-triggered necrotic symptom assays}

The BcPG3-triggered necrotic symptom assay was performed as described previously (Zhang et al., 2014). Briefly, BcPG3 protein was dissolved in $10 \mathrm{mM}$ sodium acetate buffer ( $\mathrm{pH} 4.2$ ) to a concentration of $1.5 \mu \mathrm{M}$, and this solution was infiltrated in eight leaves from three five- to six-week-old Arabidopsis plants. Pictures were taken at 5 days post-infiltration.

\section{Reactive oxygen species (ROS) measurements}

Rosette leaves of five- to six-week-old Arabidopsis plants were used. Eight leaf discs from four leaves of two plants were harvested by a disposable biopsy punch (Diameter: $4 \mathrm{~mm}$, Kai Medical), and were floated on $50 \mu \mathrm{L}$ of Milli-Q (MQ) water in a 96-well culture plate (Greiner bio-one). The plate was kept in the dark at room temperature to eliminate auto luminescence. After overnight incubation, the MQ was carefully removed without damaging the leaf discs. Leaf discs were immediately mixed with a freshly prepared assay solution (in MQ) containing $50 \mu \mathrm{M}$ luminol L-012 (Fujifilm) and $10 \mu \mathrm{g} / \mathrm{mL}$ horseradish peroxidase (Sigma). NLP24 was used at a final concentration of $1 \mu \mathrm{M}$. Subsequently, the luminescence generated by the ROS burst was measured by a microplate reader (CLARIOstar, BMG Labtech), with a programme of 60 cycles and 2 min per cycle. Data were exported and plotted with GraphPad Prism7.

\section{Acknowledgements}

We thank Birgit Kemmerling for the constructs of eYFP-tagged BIR1, BIR2, BIR3 and BIR4, and Arabidopsis seeds of bir2-1, bir3-1, bir3-2 and OE BIR3. We thank Frans E. Tax for Arabidopsis seeds of bir4-2 and bir4-3. We thank Sergio Landeo-Villanueva and Aranka M. van der Burgh for critical discussions. J.W. is supported by a PhD scholarship from the China Scholarship Council (CSC). 


\section{Supplementary information}

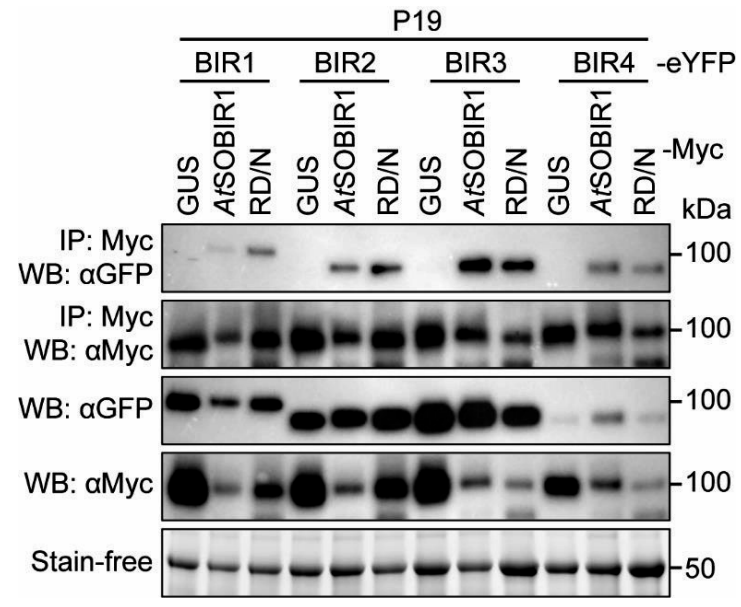

Figure S1. Kinase activity of AtSOBIR1 affects its interaction with the BIRs. C-terminally eYFPtagged BIRs were transiently co-expressed with C-terminally Myc-tagged GUS, AtSOBIR1 or AtSOBIR1RDN , in the presence of P19 in leaves of $N$. benthamiana. Proteins were extracted and subjected to IP by using Myc-affinity beads, followed by WB. aMyc was used to detect Myc-tagged proteins, and $\alpha$ GFP was used to detect eYFP-tagged BIR proteins. Rubisco bands in the stain-free panel indicate equal loading. The experiment was repeated three times with similar results, and representative results are shown. 


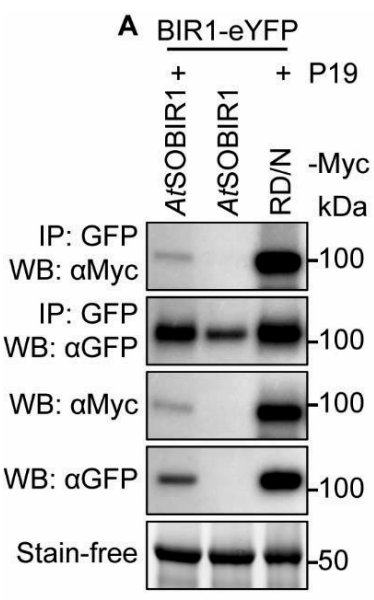

E

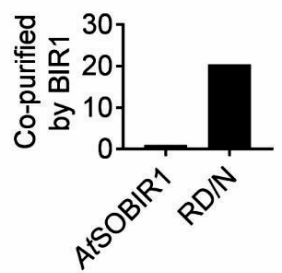

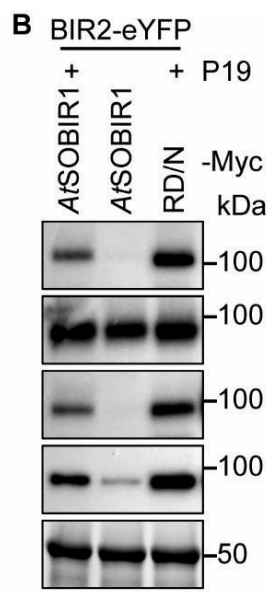

$\mathbf{F}$

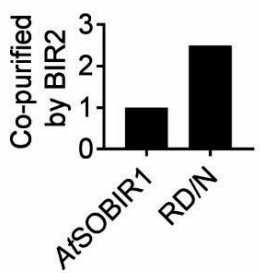

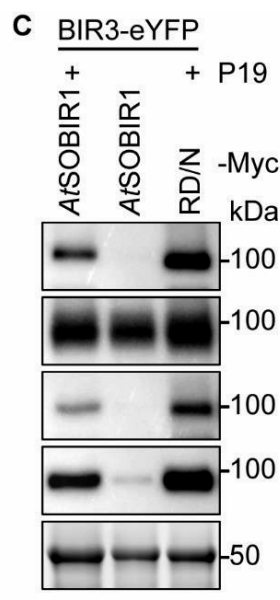

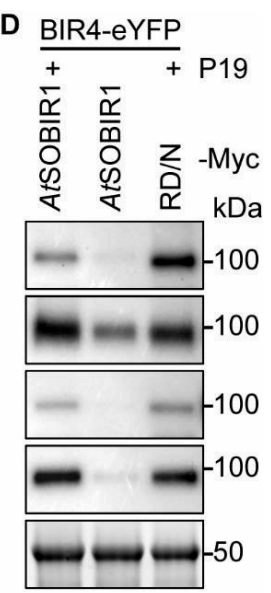

G

H

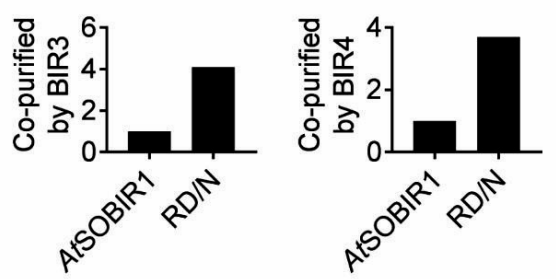

Figure S2. BIRs interact with higher amounts of kinase-inactive AtSOBIR1 in the presence of P19. (A-D) All BIR proteins interact with higher amounts of AtSOBIR1 ${ }^{R D / N}$ protein in the presence of $\mathrm{P} 19$, whereas no interaction between AtSOBIR1 and the BIRs is observed in the absence of P19. C-terminally eYFP-tagged BIRs were transiently co-expressed with AtSOBIR1-Myc in the presence or absence of $\mathrm{P} 19$, and with AtSOBIR1 $1^{\mathrm{RD} / \mathrm{N}}-\mathrm{Myc}$ in the presence of $\mathrm{P} 19$, in leaves of $N$. benthamiana. Proteins were extracted and subjected to IP by using GFP-affinity beads, followed by WB. aGFP was used to detect eYFP-tagged BIR proteins, and aMyc was used to detect AtSOBIR1-Myc and AtSOBIR1 ${ }^{\text {RDIN-Myc. }}$ Rubisco bands in the stain-free panel indicate equal loading. The experiment was repeated three times with similar results, and representative results are shown. (E-H) Quantification of the relative amounts of co-purified AtSOBIR1 and AtSOBIR1RD/N as shown in (A-D). The relative amount of co-purified AtSOBIR1 was set to 1 . 

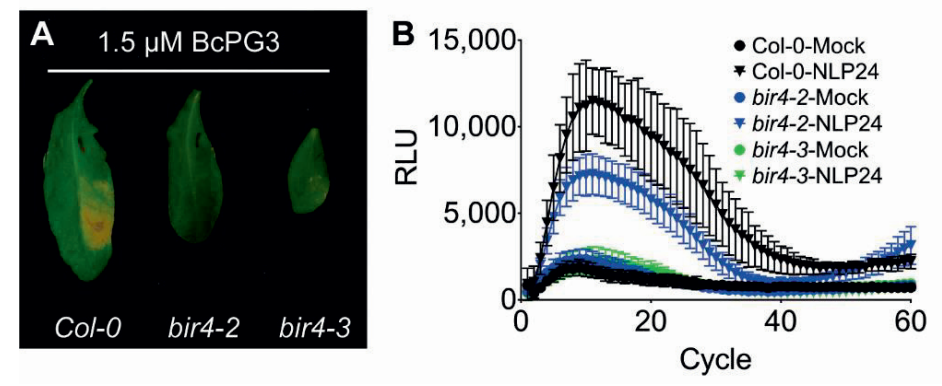

Figure S3. BIR4 is a positive regulator of RLP-mediated immune responses in Arabidopsis. (A) BIR4 is a positive regulator of the development of RLP42/BcPG3-triggered necrotic symptoms. Arabidopsis leaves were infiltrated with $1.5 \mu \mathrm{M} \mathrm{BcPG3}(\mathrm{n}=8)$, and pictures were taken at $5 \mathrm{dpi}$. Note that the development of BcPG3-triggered necrotic symptoms is suppressed in mutants of bir4. (B) BIR4 is a positive regulator of the RLP23/NLP24-triggered ROS burst. Arabidopsis leaf discs were treated with either Milli-Q water as a control (Mock) or $1 \mu$ M NLP24 $(n=8)$. Note that the NLP24-triggered ROS burst is suppressed in mutants of bir4. These experiments were repeated three times with similar results, and representative results are shown. 



\section{Chapter 3}

The bacterial effector AvrPto targets the regulatory co-receptor SOBIR1 and suppresses defence signalling mediated by the receptor-like protein $\mathrm{Cf}-4$

Jinbin $\mathrm{Wu}^{1}$, Aranka van der Burgh ${ }^{1}$, Guozhi Bi ${ }^{1 \dagger}$, Lisha Zhang ${ }^{1 \ddagger}$, James R. Alfano ${ }^{2,3}$, Gregory B. Martin ${ }^{4,5}$ and Matthieu H.A.J. Joosten ${ }^{1}$

${ }^{1}$ Laboratory of Phytopathology, Wageningen University \& Research, Droevendaalsesteeg 1, 6708 PB Wageningen, The Netherlands.

${ }^{2}$ Center for Plant Science Innovation, University of Nebraska, Lincoln, NE 68588, USA. ${ }^{3}$ Department of Plant Pathology, University of Nebraska, Lincoln, NE 68588, USA.

${ }^{4}$ Boyce Thompson Institute for Plant Research, Ithaca, NY 14853, USA.

${ }^{5}$ Section of Plant Pathology and Plant-Microbe Biology, School of Integrative Plant Science, Cornell University, Ithaca, NY 14853, USA.

tPresent address: The State Key Laboratory of Plant Genomics, Institute of Genetics and Developmental Biology, Chinese Academy of Sciences, Beijing 100101, China.

¥Present address: Center of Plant Molecular Biology (ZMBP), University of Tübingen, Auf der Morgenstelle 32, Tübingen D-72076, Germany.

A slightly modified version has been published in: Molecular Plant-Microbe Interactions (2018) 31: 75-85. 


\section{Chapter 3}

\section{Abstract}

Receptor-like proteins (RLPs) and receptor-like kinases (RLKs) are cell surface receptors that are essential for detecting invading pathogens and subsequent activation of plant defence responses. RLPs lack an intracellular kinase domain to trigger downstream signalling leading to host resistance. The regulatory RLK SOBIR1 constitutively interacts with the tomato RLP Cf-4, thereby providing Cf-4 with a kinase domain. SOBIR1 is required for Cf-4-mediated resistance to strains of the biotrophic fungal pathogen Cladosporium fulvum secreting the effector Avr4. Upon perception of this effector by the Cf-4/SOBIR1 complex, the central regulatory RLK SERK3a is recruited to the complex and defence signalling is triggered. SOBIR1 is also required for RLP-mediated resistance to bacterial, fungal and oomycete pathogens and we hypothesized that SOBIR1 is targeted by effectors of such pathogens to suppress host defence responses. In this study we show that Pseudomonas syringae pv. tomato DC3000 effector AvrPto interacts with Arabidopsis SOBIR1 and its orthologues of tomato and Nicotiana benthamiana, independent of SOBIR1 kinase activity. Interestingly, AvrPto suppresses Arabidopsis SOBIR1-induced cell death in $N$. benthamiana. Furthermore, AvrPto compromises Avr4-triggered cell death in Cf-4transgenic $N$. benthamiana, without affecting Cf-4/SOBIR1/SERK3a complex formation. Our study shows that the regulatory RLK of RLP receptors, SOBIR1, is targeted by a bacterial effector, which results in compromised defence responses. 


\section{Introduction}

The innate immune system of plants against invading pathogens consists of two layers, which are termed microbe-associated molecular pattern (MAMP)-triggered immunity (MTI) and effector-triggered immunity (ETI) (Dodds and Rathjen, 2010; Jones and Dangl, 2006). MAMPs are conserved structural components of pathogens, whereas effectors are typically in planta-induced proteins of microbial pathogens. Effectors suppress plant defence responses, thereby causing effector-triggered susceptibility (ETS) (Dodds and Rathjen, 2010; Jones and Dangl, 2006). Essentially, there are two sub-cellular locations of pathogen perception: the apoplast and the cytoplasm (Cui et al., 2009; Dodds and Rathjen, 2010; Zipfel, 2014). MAMPs and secreted effectors that end up in the apoplast are generally recognised by plasma membrane-associated pattern recognition receptors (PRRs) (Dodds and Rathjen, 2010; Zipfel, 2014). Effectors that are translocated from fungal or oomycete haustoria, which are specialised feeding structures that are formed in the host cells, or injected into the cell by the type three secretion system (TTSS) of bacteria, are perceived by cytoplasmic immune receptors (Dodds and Rathjen, 2010). These cytoplasmic receptors mostly carry a nucleotide binding site and leucine-rich repeats (NB-LRRs) (Dodds and Rathjen, 2010).

PRRs are either receptor-like kinases (RLKs) or receptor-like proteins (RLPs) (Couto and Zipfel, 2016; Zipfel, 2014). Compared to RLKs, RLPs lack an intracellular kinase domain to trigger downstream signalling. Recently it was observed that RLPs constitutively interact with the regulatory RLK SUPPRESSOR OF BAK1INTERACTING RECEPTOR-LIKE KINASE 1 (BIR1) (SOBIR1) and require SOBIR1 for their function (Gust and Felix, 2014; Liebrand et al., 2013; Liebrand et al., 2014). Arabidopsis thaliana (At) SOBIR1 was originally identified as a suppressor of bir1-1, partially rescuing bir1 knock-out plants that mount a constitutive defence response (Gao et al., 2009). AtSOBIR1 was found to function as a positive regulator of cell death, as overexpression of AtSOBIR1 triggered enhanced basal defence and reduced colonisation by the bacterial pathogen Pseudomonas syringae pv. tomato (Pst) DC3000 (Gao et al., 2009). Recently, the RLK SOMATIC EMBRYOGENESIS RECEPTOR KINASE 3 (SERK3)/BRASSINOSTEROID INSENSITIVE 1 (BRI1)ASSOCIATED RECEPTOR KINASE 1 (BAK1), further referred to as SERK3, has been reported as another suppressor of bir1-1 (Liu et al., 2016). Both SOBIR1 and SERK3 are required for bir1-1-triggered autoimmunity, and interaction was found between SOBIR1 and SERK3 in BIR1-silenced Arabidopsis (Liu et al., 2016).

The tomato (Solanum lycopersicum, SI) RLP Cf-4 confers resistance to the pathogenic 
biotrophic fungus Cladosporium fulvum, by recognition of the secreted effector Avr4 (Joosten et al., 1994; Thomas et al., 1997). It was recently shown that SERK3 is required for Cf-4 signalling and is recruited to the Cf-4/SOBIR1 complex upon recognition of Avr4 (Postma et al., 2016). Increasing evidence on signalling by RLP/SOBIR1/SERK3-containing complexes indicates that SOBIR1 constitutively forms a complex with RLPs, whereas SERK3 is specifically recruited to the RLP/SOBIR1 bipartite RLK upon ligand recognition by the RLP (Albert et al., 2015; Zhang et al., 2013a). SOBIR1 and SERK3 are also required for tomato Ve1-, I- and Brassica napus LepR3-mediated resistance to Verticillium dahliae expressing Ave1 (Liebrand et al., 2013), Fusarium oxysporum f. sp. lycopersici expressing Avr1 (Catanzariti et al., 2017) and Leptosphaeria maculans expressing AvrLm1 (Ma and Borhan, 2015), respectively, although for these particular RLPs the association of SERK3 with the RLP/SOBIR1 complex remains to be shown. SOBIR1 is also required for the functionality of various additional RLPs playing a role in immunity (Hegenauer et al., 2016; Jehle et al., 2013a; Zhang et al., 2014), although the requirement of SERK3 for the functionality of these RLPs remains currently unknown. Furthermore, SOBIR1 is involved in resistance to the oomycete pathogen Phytophthora parasitica (Peng et al., 2015) and the fungus Magnaporthe oryzae (Takahashi et al., 2016).

The Arabidopsis RLK FLAGELLIN-SENSING 2 (FLS2) confers resistance to Pst DC3000 (Gómez-Gómez and Boller, 2000; Zipfel et al., 2004). Flg22, a 22-amino-acid peptide derived from a conserved domain of bacterial flagellin, is perceived by FLS2 together with the regulatory RLK SERK3 (Chinchilla et al., 2007; Felix et al., 1999; Gómez-Gómez and Boller, 2000; Heese et al., 2007; Schulze et al., 2010; Sun et al., 2013b). Recognition of flg22 triggers a rapid defence response, including calcium spiking, reactive oxygen species (ROS) synthesis and MITOGEN-ACTIVATED PROTEIN KINASE (MAPK) activation (Boudsocq et al., 2010; Couto and Zipfel, 2016; Kadota et al., 2014). The receptor-like cytoplasmic kinase (RLCK) BOTRYTISINDUCED KINASE1 (BIK1) constitutively interacts with FLS2 and SERK3, and association with FLS2 or SERK3 is not affected in single serk3- or fls2-knock-out mutants, respectively (Lu et al., 2010; Zhang et al., 2010). However, flg22-induced BIK1 phosphorylation depends on both FLS2 and SERK3, as BIK1 phosphorylation does not take place in fls2 or serk3 mutants (Lu et al., 2010). SERK3 phosphorylates BIK1 that in turn phosphorylates SERK3 and FLS2 (Lin et al., 2014; Lu et al., 2010). After flg22 perception, BIK1 is released from the FLS2/BAK1 complex and triggers early defence responses (Lu et al., 2010; Zhang et al., 2010).

Pathogenic bacteria inject their effectors into the host cells via the TTSS to suppress MTI by directly interfering with PRR function or with downstream signalling pathways 
(Bi and Zhou, 2017; Couto and Zipfel, 2016; Lee et al., 2013; Tang et al., 2017). For example, the effector AvrPto is injected into host cells by Pst DC3000 and localizes to the plasma membrane (Shan et al., 2000). AvrPto targets FLS2 and suppresses the phosphorylation of its kinase domain (Xiang et al., 2008). AvrPto likely also targets SERK3, thereby inhibiting BIK1 phosphorylation and MAPK activation (He et al., 2006; Lu et al., 2010; Shan et al., 2008; Xiang et al., 2011).

The resistance gene Pto (for resistance against Pst race 0) encodes an RLCK with serine-threonine specificity (Loh and Martin, 1995), which competes with FLS2 for AvrPto to trigger ETI (Xiang et al., 2008). In tomato, Pto confers resistance to Pst DC3000 carrying AvrPto (Martin et al., 1993) in a Pseudomonas resistance and fenthion (Prf)-dependent manner. Transient overexpression of AvrPto in Pto/Prftransgenic Nicotiana benthamiana causes a hypersensitive response (HR) (Balmuth and Rathjen, 2007; Salmeron et al., 1994; Scofield et al., 1996). However, AvrPto does not affect association between Pto and Prf (Mucyn et al., 2006).

SOBIR1 is generally required for RLP-mediated resistance to bacterial, fungal and oomycete pathogens (Albert et al., 2015; Catanzariti et al., 2017; Hegenauer et al., 2016; Jehle et al., 2013a; Liebrand et al., 2013; Ma and Borhan, 2015; Peng et al., 2015; Postma et al., 2016; Takahashi et al., 2016; Zhang et al., 2014; Zhang et al., 2013a). Therefore, we hypothesized that this regulatory RLK is targeted by cytoplasmic effectors of such pathogens in order to suppress host defence responses. Here we show that tomato SOBIR1, its paralogue SISOBIR1-like and its orthologues from Arabidopsis and $N$. benthamiana all interact with AvrPto in a manner independent of SOBIR1 kinase activity. In addition, AvrPto suppresses AtSOBIR1-induced constitutive immunity in $N$. benthamiana. Furthermore, AvrPto was found to suppress the Cf4/Avr4-triggered HR without affecting Cf-4/SISOBIR1/SISERK3a complex formation.

\section{Results}

\section{AvrPto interacts with SOBIR1 in planta}

To investigate whether AvrPto interacts with SOBIR1, a binary construct containing Cterminally haemagglutinin (HA) epitope-tagged AvrPto was generated. AvrPto-HA was co-infiltrated with C-terminally tagged SISOBIR1-eGFP (Liebrand et al., 2013) in N. benthamiana by Agrobacterium-mediated transient expression (agro-infiltration). AvrPto-HA was also co-infiltrated with C-terminally tagged SISERK3a-eGFP as a positive control, because AtSERK3 was earlier reported to be targeted by AvrPto (Shan et al., 2008). Co-immunoprecipitation (co-IP) experiments reveal that AvrPto-HA copurifies with SISOBIR1-GFP upon pull-down of the latter by using GFP beads (Fig. 1A). 


\section{Chapter 3}

An interaction between SISERK3a and AvrPto was also observed (Fig. 1A). In a reciprocal experiment in which SISOBIR1, SISERK3a and GUS were fused to HA and co-infiltrated with AvrPto-eGFP, a pull-down of SISOBIR1 and SISERK3a using HA beads also resulted in co-purification of AvrPto-eGFP (Fig. 1B).
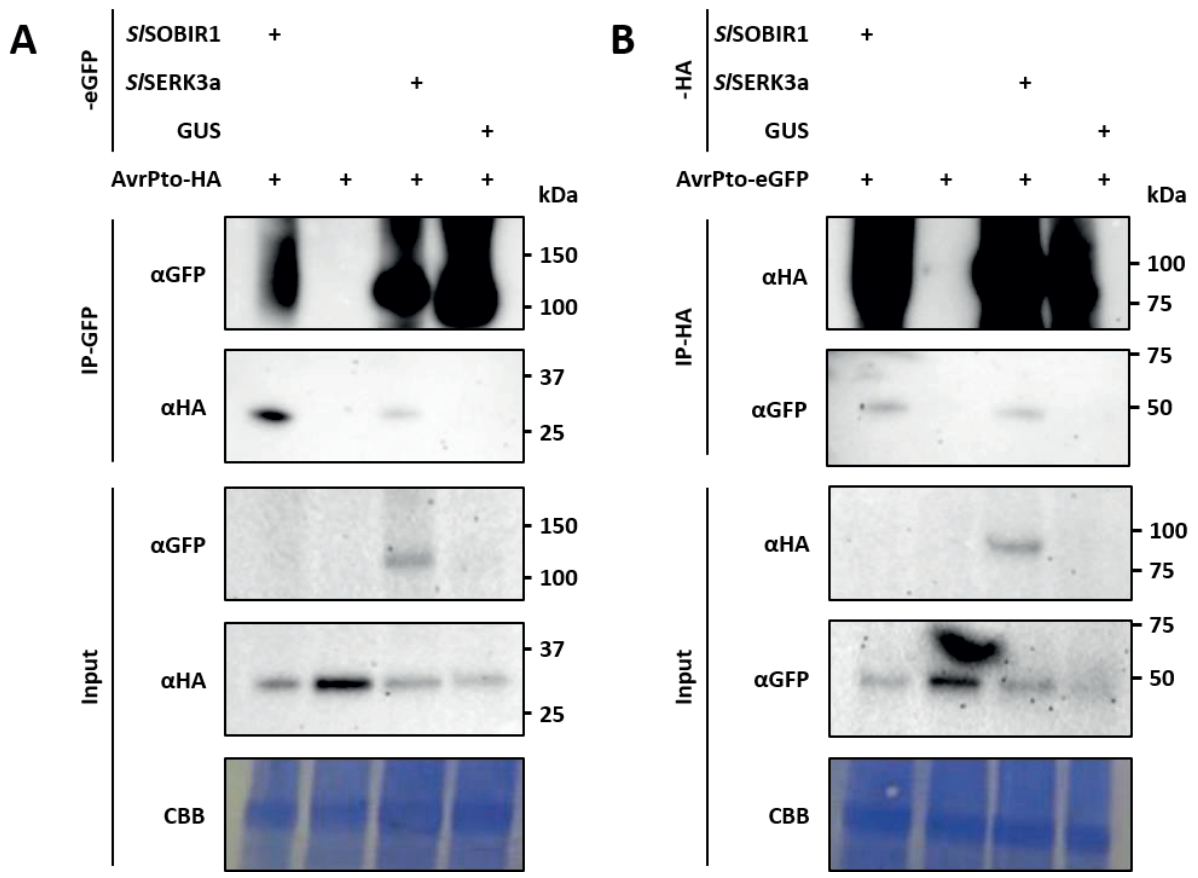

Figure 1. AvrPto interacts with SISOBIR1 in planta. (A) AvrPto-HA interacts with SISOBIR1-eGFP. C-terminally eGFP-tagged SISOBIR1, SISERK3a and GUS were co-expressed with AvrPto-HA by agroinfiltration in $N$. benthamiana. Two days later, total proteins were extracted and subjected to immunoprecipitation using GFP_TrapA affinity beads. Total proteins (Input) and immunoprecipitated proteins (IP) were subjected to SDS-PAGE and blotted to PVDF membrane. Blots were probed with aGFP antibodies to detect the immunoprecipitated eGFP-tagged proteins, and with aHA antibodies to detect co-precipitated AvrPto-HA. CBB, coomassie brilliant blue. Experiments were performed at least three times and representative figures are shown. (B) AvrPto-eGFP interacts with SISOBIR1-HA and SISERK3a-HA. C-terminally HA-tagged SISOBIR1, SISERK3a and GUS were co-expressed with AvrPto-eGFP in $N$. benthamiana. Two days later, total proteins were extracted and subjected to immunoprecipitation using HA magnetic beads. Total proteins (Input) and immunoprecipitated proteins (IP) were subjected to SDS-PAGE and blotted to PVDF membrane. Blots were probed with aHA antibodies to detect the immunoprecipitated HA-tagged proteins, and with aGFP antibodies to detect co-precipitated AvrPto-eGFP. CBB, coomassie brilliant blue. Experiments were performed at least three times and representative figures are shown.

In tomato, the SISOBIR1 paralogue SISOBIR1-like shares a high similarity in amino 
acid sequence with SISOBIR1, and in N. benthamiana two clear SOBIR1 paralogues are present (Liebrand et al., 2013). In addition, the function of SOBIR1 appears to be highly conserved, as both AtSOBIR1 and SISOBIR1 interact with Cf-4 (Liebrand et al., 2013), and AtSOBIR1 is able to complement the loss of Avr4-triggered cell death in NbSOBIR1(-like)-silenced Cf-4 transgenic N. benthamiana plants (Bi et al., 2016; Liebrand et al., 2013). To study if the SOBIR1 paralogue and its orthologues are also targeted by AvrPto, C-terminally eGFP-tagged SISOBIR1-like and NbSOBIR1 were generated and, together with SISOBIR1-eGFP and AtSOBIR1-eGFP (Bi et al., 2016; Liebrand et al., 2013), were co-infiltrated with AvrPto-HA in N. benthamiana. Co-IP experiments reveal that in all cases AvrPto co-purifies with the SOBIR1 variants (Fig. 2).

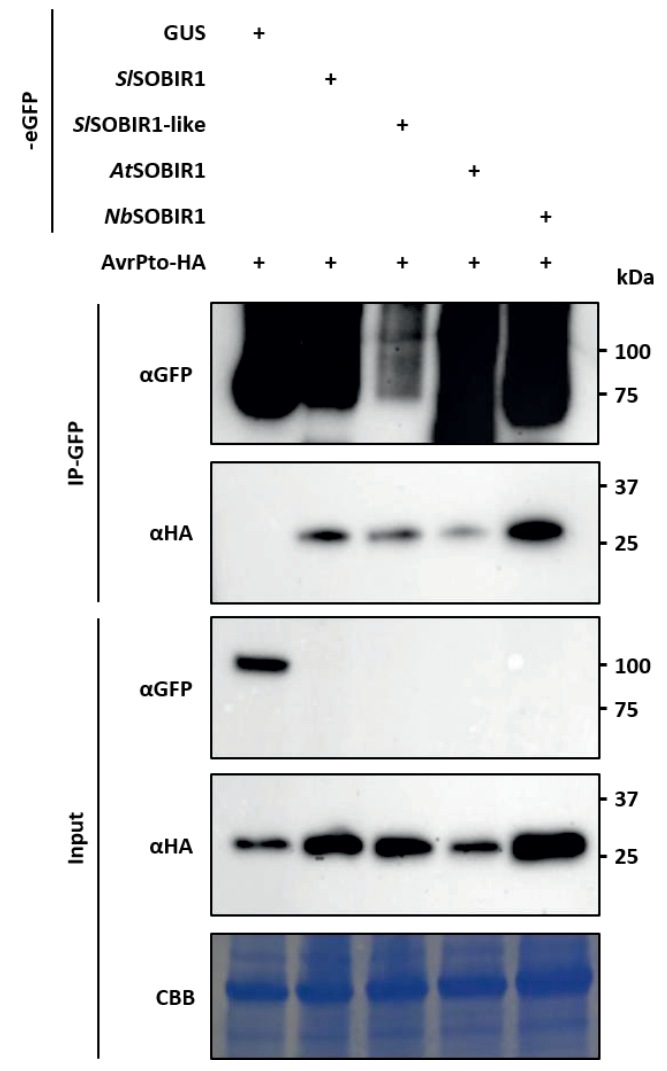

Figure 2. AvrPto interacts with SISOBIR1, SISOBIR1-like, AtSOBIR1 and NbSOBIR1. C-terminally eGFP-tagged GUS, SISOBIR1, SISOBIR1-like, AtSOBIR1 and NbSOBIR1 were co-expressed with AvrPto-HA by agroinfiltration in N. benthamiana. Two days later, total proteins were extracted and subjected to immunoprecipitation using GFP_TrapA affinity beads. Total proteins (Input) and immunoprecipitated proteins (IP) were subjected to SDS-PAGE and blotted to PVDF membrane. Blots were probed with aGFP antibodies to detect the immunoprecipitated eGFP-tagged proteins, and with $\mathrm{aHA}$ antibodies to detect co-precipitated AvrPto-HA $\mathrm{CBB}$, coomassie brilliant blue. Experiments were performed at least three times and representative figures are shown.

\section{Kinase activity of SOBIR1 is not required for its interaction with AvrPto}

SOBIR1 is a so-called "RD" kinase and by its constitutive interaction with RLPs, bipartite RLKs are formed. In this bi-partite RLK, SOBIR1 is thought to provide the 
interacting RLP with a kinase domain to initiate defence signalling upon ligand recognition by the RLP (Gust and Felix, 2014; Liebrand et al., 2014). It has been reported that AvrPto targets Pto and FLS2, while the interaction is dependent on kinase activity of these two targets (Xiang et al., 2008; Xing et al., 2007). To determine if kinase activity of SOBIR1 is required for its interaction with AvrPto, eGFP-tagged wild-type and kinase-dead SOBIR1 variants (mutated in the catalytic aspartate (D) of the kinase domain) of Arabidopsis (AtSOBIR1 ${ }^{\mathrm{D} 489 \mathrm{~N}}$ ) and tomato SOBIR1 (SISOBIR1 ${ }^{\mathrm{D} 473 \mathrm{~N}}$ ) (Liebrand et al., 2013) were co-infiltrated with AvrPto-HA. Co-IP experiments reveal that AvrPto co-purifies with all SOBIR1 variants upon their purification using GFP beads, indicating that kinase activity of SOBIR1 is not required for its interaction with AvrPto (Fig. 3).

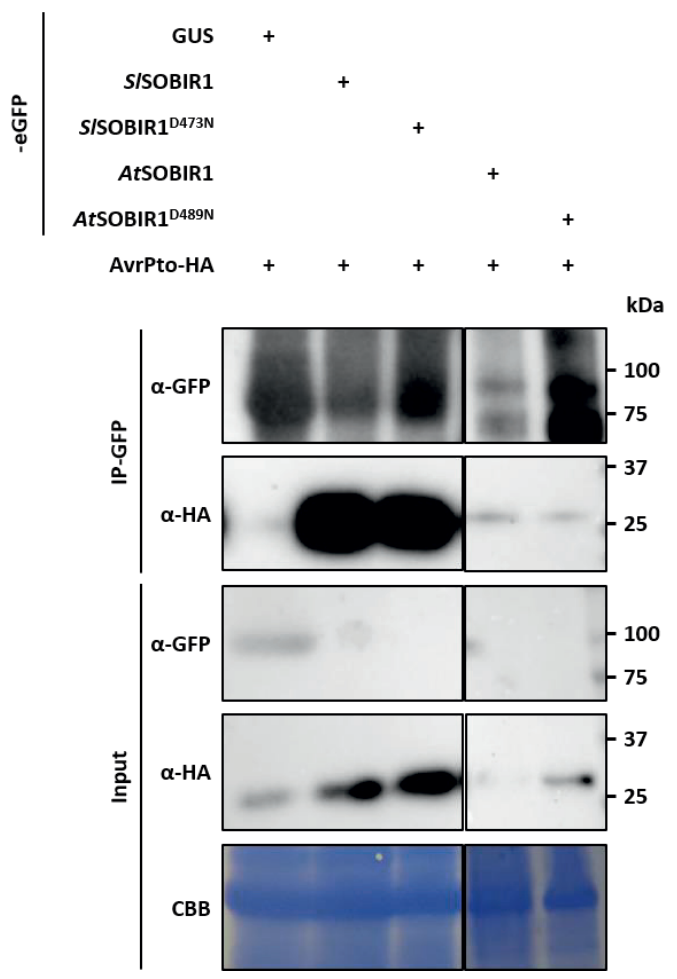

Figure 3. Kinase activity of SISOBIR1 and AtSOBIR1 is not required for their interaction with AvrPto. C-terminally eGFP-tagged GUS, SISOBIR1, kinase-dead SISOBIR1D473N, AtSOBIR1 and kinase-dead AtSOBIR1D489N were co-expressed with AvrPto-HA by agro-infiltration in $N$. benthamiana. Two days later, total proteins were extracted and subjected to immunoprecipitation using GFP_TrapA affinity beads. Total proteins (Input) and immunoprecipitated proteins (IP) were subjected to SDS-PAGE and blotted to PVDF membrane. Blots were probed with aGFP antibodies to detect the immunoprecipitated eGFP-tagged proteins, and with aHA antibodies to detect coprecipitated AvrPto-HA. CBB, coomassie brilliant blue. Experiments were performed at least three times and representative figures are shown.

\section{Overexpression of AtSOBIR1 induces constitutive immunity, which requires a functional kinase domain}

SOBIR1 is a positive regulator of immunity (Gao et al., 2009), and we anticipated that the protein would constitutively induce an immune response, visualised as cell death, when it accumulates at relatively high levels. To investigate this, eGFP-tagged AtSOBIR1, SISOBIR1, SISOBIR1-like and NbSOBIR1 were transiently overexpressed 

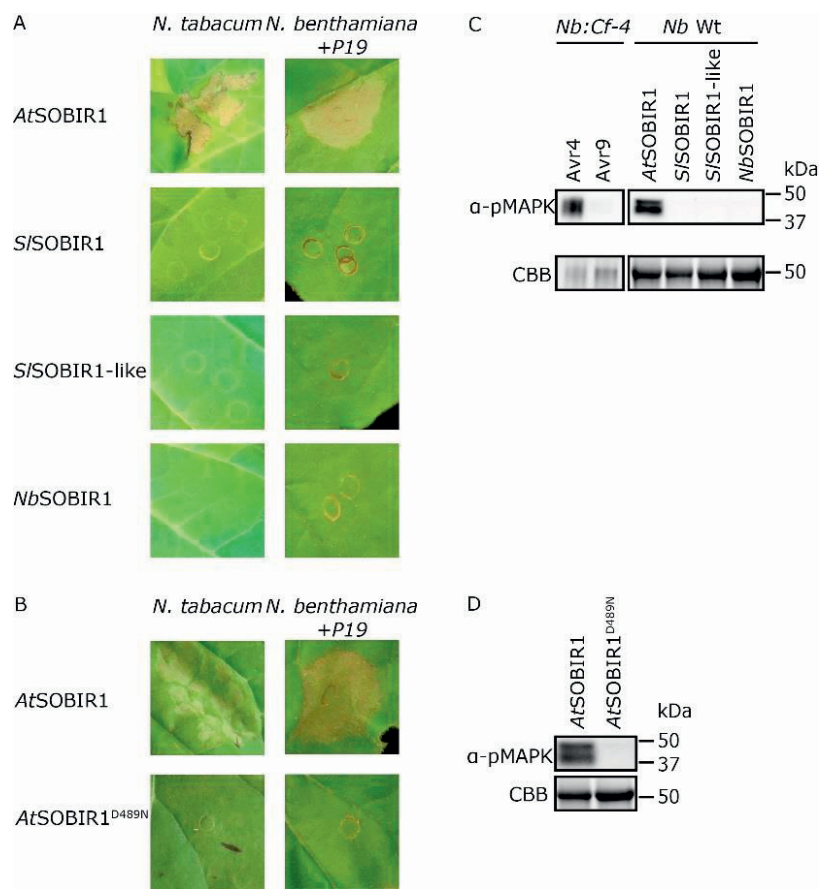

Figure 4. Constitutive immunity induced by overexpression of AtSOBIR1 is dependent on a functional kinase domain. (A) Transient expression of AtSOBIR1 induces cell death in N. tabacum and $N$. benthamiana. C-terminally eGFP-tagged AtSOBIR1, SISOBIR1, SISOBIR1-like and NbSOBIR1 were transiently expressed by agro-infiltration at an OD of 1 ; in $N$. benthamiana all constructs were coexpressed with the silencing suppressor $\mathrm{P} 19$ at an OD of 1. Pictures were taken at 2 days post-infiltration (dpi) for N. tabacum and at 3 dpi for N. benthamiana. See also Supplementary Fig. S1A. (B) Transient expression of C-terminally eGFP-tagged kinase-dead AtSOBIR1 ${ }^{D 489 N}$ does not induce cell death in $N$. tabacum or $N$. benthamiana. The experiment was performed as described for panel A. See also Supplementary Fig. S1A. (C) MAPKs are activated upon Avr4 recognition by Cf-4, and upon overexpression of AtSOBIR1. Left, $2 \mu \mathrm{M}$ of Avr4 or Avr9 protein was infiltrated in Cf-4 transgenic $N$. benthamiana plants and after $15 \mathrm{~min}$ total protein was extracted and analysed for MAPK activation using anti-p42/p44-erk antibody. Right, C-terminally eGFP-tagged AtSOBIR1, SISOBIR1, SISOBIR1-like and NbSOBIR1 were transiently co-expressed with P19 in N. benthamiana. At 2 dpi total protein was extracted and analysed for MAPK activation using anti-p42/p44-ERK antibody. (D) MAPKs are not

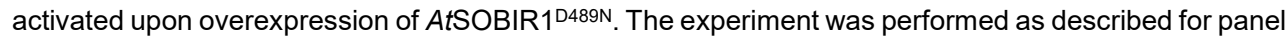
C. Experiments were performed at least three times and representative figures are shown.

in leaves of $N$. tabacum by agro-infiltration. Interestingly, only overexpression of AtSOBIR1 induced cell death, which was visible within two to three days after agroinfiltration (Fig. 4A). The other SOBIR1 variants that were tested did not exhibit this constitutive immunity symptom. Although protein accumulation was not tested in $N$. tabacum, the same constructs were expressed in N. benthamiana and resulted in clear 
protein accumulation for all constructs (discussed below) (Supplementary Fig. S1A). To determine if kinase activity of AtSOBIR1 is specifically required to induce constitutive immunity, eGFP-tagged AtSOBIR1 ${ }^{\mathrm{D} 489 \mathrm{~N}}$ was transiently overexpressed in leaves of $N$. tabacum (Fig. 4B). This kinase-dead SOBIR1 mutant did not induce cell death upon its transient overexpression, indicating that a functional kinase domain is required for AtSOBIR1 to induce constitutive immunity in $N$. tabacum. This suggests that the constitutive immune response is triggered as a result of perturbation of the immune system of the plant, through constitutive activation of downstream immune signalling components.

The same constructs were tested for constitutive immune activation in $N$. benthamiana. No cell death was observed for any of the SOBIR1 variants when expressed alone (data not shown), although all proteins do accumulate (Supplementary Fig. S1A and B). However, when co-expressed with the silencing suppressor P19 (Voinnet et al., 2015), cell death could be observed for AtSOBIR1 at two to three days after infiltration, and again not for any of the other tested SOBIR1 variants (Fig. 4A, Supplementary Fig. S1). N. tabacum is generally more sensitive to overexpression of immunity-related proteins, and probably therefore does not require co-expression of a silencing suppressor to provoke AtSOBIR1 constitutive immunity (van der Hoorn et al., 2000; Zhang et al., 2013b).

To obtain additional support that this cell death phenotype indeed reflects an immune response, we analysed MAPK activation, which is a key downstream step in defence activation (Stulemeijer et al., 2007; Xu et al., 2014). Upon Avr4 recognition by Cf-4 in stable transgenic $N$. benthamiana expressing tomato $C f-4$, immune blots showed a typical MAPK activation pattern (Fig. 4C). Notably, induction of cell death upon AtSOBIR1 overexpression in N. benthamiana also coincided with MAPK activation (Fig. $4 C$ and 4D). In conclusion, these data show that the cell death caused by AtSOBIR1 is dependent on its kinase activity and represents a constitutive immune response.

\section{AvrPto suppresses AtSOBIR1-induced constitutive immunity}

To determine whether AvrPto is able to suppress the cell death response induced by overexpression of AtSOBIR1, AvrPto-eGFP and GUS-eGFP were co-infiltrated with AtSOBIR1-eGFP and P19 in N. benthamiana. Co-infiltration of GUS-eGFP does not affect AtSOBIR1-induced cell death, whereas the cell death response is strongly suppressed by AvrPto-eGFP, indicating that AtSOBIR1-induced constitutive immunity is suppressed by AvrPto (Fig. 5). 


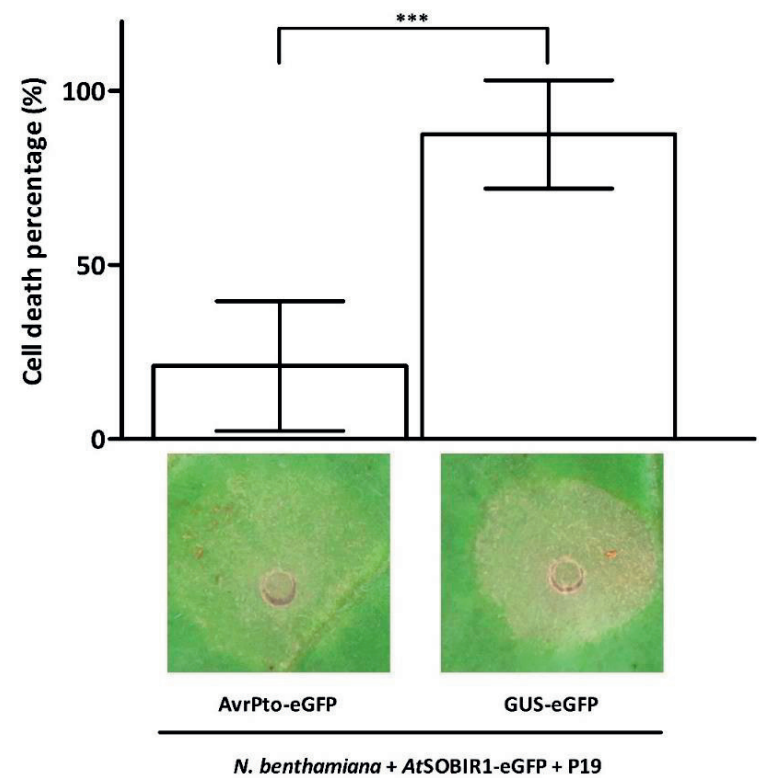

Figure 5. Constitutive immunity induced by overexpression of AtSOBIR1 is suppressed by AvrPto. AtSOBIR1-eGFP and P19 were coexpressed with GUS-eGFP or AvrPtoeGFP by agro-infiltration in $N$. benthamiana at an OD of 1 . Pictures were taken at $4 \mathrm{dpi}$. Cell death at $4 \mathrm{dpi}$ was scored by its intensity. The means of the cell death percentages are shown. Asterisks indicate a statistically significant difference according to a Student's $t$-test $\left.{ }^{* * *} P<0.0001\right)$, with $\mathrm{n}$ $=20$. Experiments were performed at least three times and representative figures are shown.

\section{AvrPto suppresses the Avr4-triggered cell death in Cf-4-transgenic $\mathrm{N}$. benthamiana plants}

SOBIR1 is required for the Cf-4-mediated cell death upon recognition of Avr4 (Liebrand et al., 2013). Furthermore, kinase activity of SOBIR1 is necessary for Cf-4 function as a kinase-inactive variant of AtSOBIR1 fails to complement the loss of Avr4-triggered cell death in NbSOBIR1(-like)-silenced Cf-4-transgenic N. benthamiana plants (Bi et al., 2016; Liebrand et al., 2013).

To study if AvrPto is able to suppress the Avr4-triggered cell death through suppression of SOBIR1-dependent defence signalling, AvrPto-eGFP and GUS-eGFP were transiently overexpressed in Cf-4-transgenic $N$. benthamiana plants, two days before agro-infiltration of Avr4. Figure 6 shows that cell death triggered by Avr4 is strongly suppressed upon infiltration of AvrPto-eGFP, when compared to infiltration of GUSeGFP. Together with the observation that AvrPto suppresses AtSOBIR1-induced constitutive immunity (Fig. 5), the suppression of Avr4-triggered cell death by AvrPto indicates that AvrPto compromises Avr4-triggered cell death through suppression of SOBIR1-mediated defence signalling. 


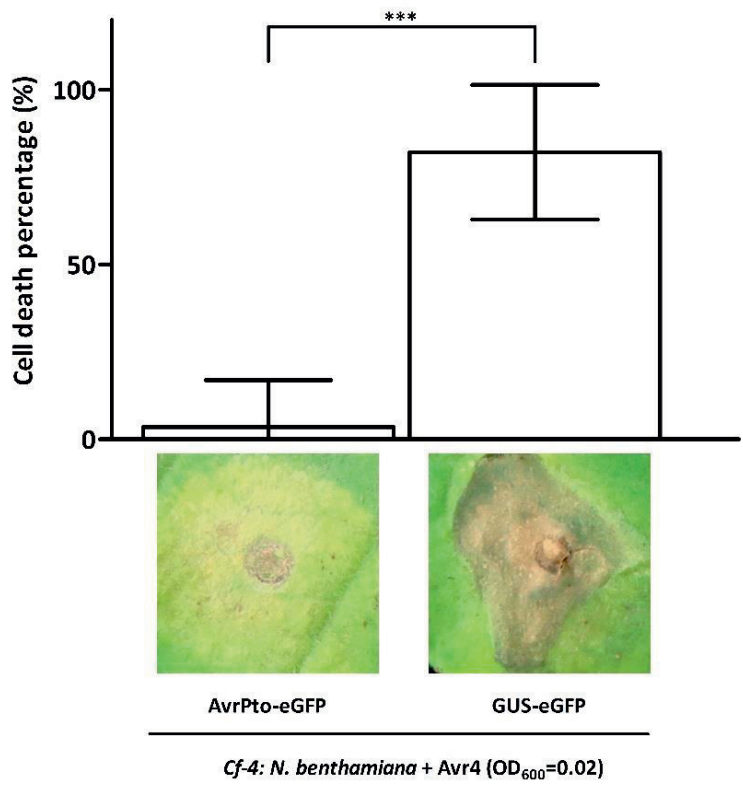

Figure 6. The Avr4-triggered cell death in $\mathrm{Cf}-4$ transgenic $N$. benthamiana plants is suppressed by AvrPto. AvrPto-eGFP and GUSeGFP were expressed at an OD of 1 in $C f-4$ transgenic $N$. benthamiana plants at two days before agro-infiltration of Avr4 at an OD of 0.02 . Pictures were taken at 5 dpi of Avr4 infiltration. Cell death at 5 dpi was scored by the intensity. The means of the cell death percentages are shown. Asterisks indicate a statistically significant difference according to a Student's ttest $\left({ }^{* *} \mathrm{P}<0.0001\right)$, with $\mathrm{n}=15$. Experiments were performed at least three times and representative figures are shown.

\section{AvrPto does not affect Cf-4/SISOBIR1/SISERK3a complex formation}

Recently it was shown that SISERK3a is involved in the Cf-4-triggered signalling pathway (Postma et al., 2016). SOBIR1 constitutively interacts with Cf-4 (Liebrand et al., 2013), whereas SISERK3a interacts with Cf-4 in an Avr4-dependent manner, as SISERK3a is specifically recruited to the Cf-4/SISOBIR1 complex in the presence of Avr4 (Postma et al., 2016). Silencing of either NbSOBIR1(-like) or NbSERK3a/b compromises the Avr4-triggered cell death (Liebrand et al., 2013; Postma et al., 2016).

To study the effect of AvrPto on Cf-4/SISOBIR1/SISERK3a complex formation, AvrPtoHA was co-infiltrated with Cf-4-eGFP, SISOBIR1-HA and SISERK3a-Myc, followed by infiltration of Avr4 protein. Figure 7 shows that Cf-4-eGFP and SISOBIR1-HA are invisible in the input, but are readily detectable in the immunoprecipitate, which is consistent with our earlier published studies (Liebrand et al., 2013; Postma et al., 2016). Co-IP experiments reveal that AvrPto does not affect the interaction between Cf-4 and SISOBIR1. Moreover, the recruitment of SISERK3a to the Cf-4/SISOBIR1 complex upon infiltration of Avr4 protein is not affected (Fig. 7). These results indicate that AvrPto does not suppress the Avr4-triggered cell death by hampering Cf4/SISOBIR1/SISERK3a complex formation upon Avr4 recognition by Cf-4. 


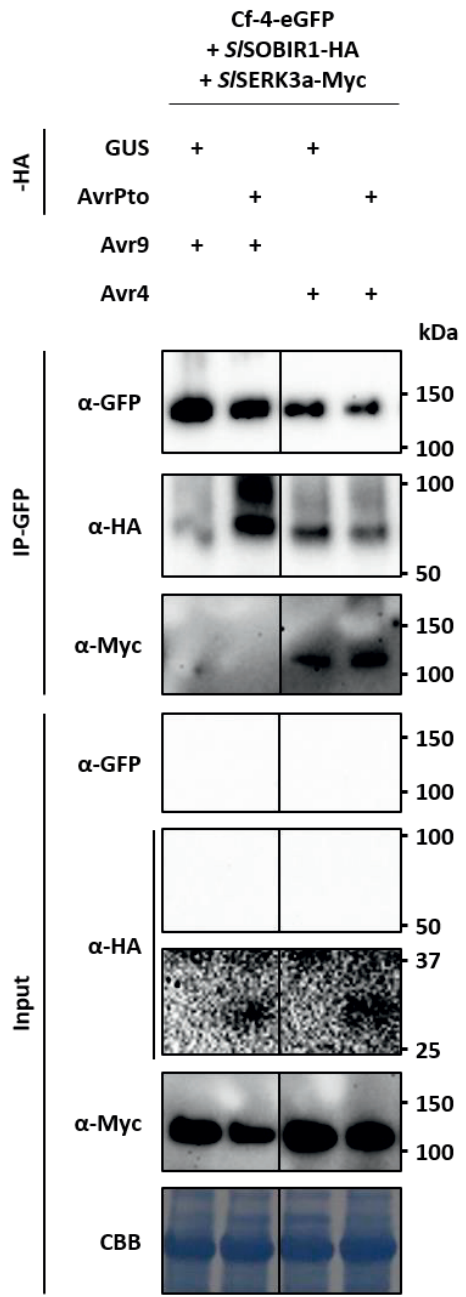

Figure 7. Avr4-mediated SISERK3a recruitment to the Cf4/SISOBIR1 complex is not affected by AvrPto. Cf-4eGFP, SISOBIR1-HA and SISERK3a-Myc were coexpressed with either GUS-HA or AvrPto-HA by agroinfiltration in N. benthamiana. Two days later, Avr4 or Avr9 protein $(2 \mu \mathrm{M})$ was infiltrated in the same area and leaves were harvested 30 min later. Total proteins were extracted and subjected to immunoprecipitation using GFP_TrapA affinity beads. Total proteins (Input) and immunoprecipitated proteins (IP) were subjected to SDS-PAGE and blotted to PVDF membrane. Blots were probed with aGFP antibodies to detect immunoprecipitated Cf-4-eGFP, with aHA antibodies to detect co-precipitated SISOBIR1-HA, and with aMyc antibodies to detect co-precipitated SISERK3a-Myc. The lower aHA panel from the input shows an overexposed blot to reveal accumulation of AvrPto-HA. CBB, coomassie brilliant blue. Experiments were performed at least three times and representative figures are shown.

\section{Discussion}

In this study, we provide evidence that AvrPto interacts with AtSOBIR1 and its orthologues from tomato and $N$. benthamiana in a manner independent of SOBIR1kinase activity. Overexpression of AtSOBIR1 induces constitutive immunity, which is suppressed by AvrPto. In addition, AvrPto also suppresses Cf-4/Avr4-triggered cell death without affecting Cf-4/SISOBIR1/SISERK3a complex formation.

\section{AtSOBIR1 induces a constitutive immune response}

AtSOBIR1 is a functional orthologue of NbSOBIR1 (Liebrand et al., 2013). In contrast to NbSOBIR1 and SISOBIR1, overexpression of AtSOBIR1 in N. tabacum or N. 
benthamiana induces constitutive immunity, which is observed as cell death (Fig. 4A). This observation is in agreement with the cell death observed upon overexpression of AtSOBIR1 in Arabidopsis (Gao et al., 2009). The lack of constitutive immune activity of $N b S O B I R 1$ and SISOBIR1 could be an effect of SOBIR1 signalling regulation in $N$. tabacum and N. benthamiana. The signalling capacity of immune receptors is tightly regulated, as immune homeostasis has to be maintained and its de-regulation may be lethal (Couto et al., 2016).

A way of regulating immune signalling activity is at the level of phosphorylation of the kinase domain of the signalling components. For instance, the Arabidopsis phosphatases PP2As and PP2C38, associating with SERK3 and BIK1, respectively, were found to negatively regulate the activity of their target kinases (Couto et al., 2016; Segonzac et al., 2014). Potential intrinsic differences between phosphatases from Arabidopsis and Solanaceous plants, involved in negatively regulating SOBIR1 signalling, could explain the lack of constitutive immune activity of SISOBIR1 or NbSOBIR1, in contrast to AtSOBIR1, when expressed in Solanaceous plants. It is likely that Solanaceous plants can fine-tune the activity of endogenous versions of SOBIR1 in a more controlled manner than the activity of heterologously expressed AtSOBIR1. Thus, it could be that endogenous phosphatases of $N$. tabacum and $N$. benthamiana can properly suppress Solanaceous SOBIR1 immune signalling, whereas these phosphatases might for example have lower affinity for the more distantly related orthologue AtSOBIR1. This could result in a higher basal level of phosphorylation of the kinase domain of AtSOBIR1. This higher level of phosphorylation might perturb the immune system of the plant and trigger a constitutive immune response in $N$. tabacum and $N$. benthamiana, which is also reflected by constitutive activation of MAPK signalling (Fig. 4C).

\section{AvrPto suppresses Cf-4/SISOBIR1/SISERK3a-triggered cell death, without affecting complex formation}

AvrPto has been reported to suppress cell death triggered by Pst strain T1 in N. benthamiana (Kang et al., 2004). In addition, AvrPto is able to suppress the flg22/elf18/elf26-triggered immune response, the latter two are fragments of the bacterial MAMP ELONGATION FACTOR-TEMPERATURE UNSTABLE (EF-Tu) that is perceived by the RLK EF-Tu RECEPTOR (EFR) (Shan et al., 2008; Xiang et al., 2008; Zipfel et al., 2006). Here we show that AvrPto is able to suppress AtSOBIR1-induced constitutive immunity (Fig. 5), as well as the Cf-4/Avr4-triggered cell death (Fig. 6). Previously, it was reported that AvrPto fails to suppress the cell death triggered by Cf9/Avr9 (Kang et al., 2004). Cf-9 also interacts with SOBIR1 (Liebrand et al., 2013) and we anticipate that Cf-9 requires SOBIR1 for its function. The absence of AvrPto- 
mediated suppression of Cf-9/Avr9-induced cell death might be caused by the use of a lower amount of Agrobacterium expressing AvrPto $\left(\mathrm{OD}_{600}=0.4\right.$, as compared to $\mathrm{OD}$ $=1.0$ in our studies). Furthermore, Kang et al. (2004) co-infiltrated AvrPto with Cf9/Avr9, whereas in our study AvrPto was infiltrated two days before infiltration of Avr4 in Cf-4 transgenic $N$. benthamiana.

It has been shown that AvrPtoB, which is another TTSS effector of Pst DC3000 (Pedley and Martin, 2003), and AvrPto both interact with FLS2 and SERK3, whereas only the association of AvrPtoB with FLS2 gets stronger upon flg22 treatment (Göhre et al., 2008; Shan et al., 2008; Xiang et al., 2008). In addition, the flg22-induced FLS2-SERK3 interaction is suppressed by both AvrPtoB and AvrPto (Shan et al., 2008). Furthermore, the flg22-induced dissociation of BIK1 from FLS2 and SERK3 is also suppressed by AvrPto (Lu et al., 2010; Zhang et al., 2010). In view of developmental regulation, SERK3 is involved in the BRASSINOLIDE (BR)-triggered signalling pathway, since serk3 mutants show reduced sensitivity to BR ( $\mathrm{Li}$ et al., 2002). SERK3 constitutively interacts with the BR receptor BRI1 ( $\mathrm{Li}$ et al., 2002), and this interaction is also suppressed by AvrPto (Shan et al., 2008).

Altogether, these observations indicate that AvrPto suppresses flg22-triggered immunity by interrupting PRR complex formation with the essential co-receptor SERK3, and suppresses the formation of BRI1/SERK3 complex that is involved in BR-mediated developmental regulation (Shan et al., 2008). In this study, we show that AvrPto is able to suppress Cf-4/Avr4-triggered cell death, without affecting the constitutive Cf4/SISOBIR1 complex and the Avr4-induced recruitment of SISERK3a to the Cf4/SISOBIR1 complex (Fig. 6 and 7). As AvrPto interacts with both SISOBIR1 and SISERK3 (Fig. 1), AvrPto might interfere in the phosphorylation status of SOBIR1 and/or SERK3 to suppress Cf-4/Avr4-triggered cell death (Fig. 6).

\section{TTSS effectors interfere in host protein phosphorylation}

Effector-mediated modifications of defence-related proteins is an effective way to suppress the immune response, and various TTSS effectors have been shown to interfere with the phosphorylation status of important host kinase proteins, including PRRs, RLCKs and MPKs (Bi and Zhou, 2017; Couto and Zipfel, 2016; Lee et al., 2013; Macho and Zipfel, 2015; Tang et al., 2017).

Pseudomonas HopAO family members all have tyrosine phosphatase activity, which is required for pathogen virulence (Bretz et al., 2003; Castaneda-Ojeda et al., 2017). For instance, the phosphatase catalytic activity of HopAO1 is required for suppressing callose deposition and MAPK activation upon bacterial colonisation of Arabidopsis 
(Castaneda-Ojeda et al., 2017; Macho et al., 2014; Underwood et al., 2007). Furthermore, it was reported that the kinase domain of EFR is targeted by HopAO1, which directly dephosphorylates EFR kinase activity, but does not affect the elf18induced interaction between EFR and SERK3 (Macho et al., 2014).

The regulatory RLK SERK3 is also a central target of bacterial effectors (Yasuda et al., 2017). For instance, HopF2 targets SERK3 to suppress BIK1 phosphorylation, thereby compromising MAPK activation (Wu et al., 2011; Zhou et al., 2014). In addition, AvrPtoB targets and dephosphorylates SERK3 to suppress FLS2 signalling (Cheng et al., 2011). FLS2 and EFR are also targets of AvrPtoB, which leads to degradation of these two receptors through ubiquitination (Göhre et al., 2008). However, AvrPtoB does not affect the kinase activity of FLS2 and EFR (Göhre et al., 2008). Furthermore, AvrPtoB also targets Arabidopsis LysM-RLK CHITIN RECEPTOR KINASE1 (CERK1) and degrades the protein, and this effector also appears to suppress CERK1 kinase activity (Gimenez-lbanez et al., 2009). Additionally, kinase activity of the AvrPtoB TOMATO-INTERACTING 9 (Bti9) protein, the closest tomato homologue of CERK1, is also suppressed by AvrPtoB (Zeng et al., 2012).

Cytoplasmic RLCKs, which play a role downstream of PRRs, are phosphorylated by ligand-activated PRRs and in their turn trans-phosphorylate the kinase domain of these PRRs (Couto and Zipfel, 2016). Phosphorylation of the RLCK AVRPPHB SUSCEPTIBLE 1 (PBS1) (Swiderski and Innes, 2001) is required for its interaction with RESISTANCE TO PSEUDOMONAS SYRINGAE 5 (RPS5) (Ade et al., 2007; Warren et al., 1998). PBS1 is cleaved by HopAR1 (AvrPphB) from Pst DC 3000 to activate RPS5-mediated auto immunity (Ade et al., 2007; Shao et al., 2003). In addition, HopAR1 also cleaves BIK1 to suppress the flg22-induced signalling pathway (Zhang et al., 2010). Furthermore, BIK1 is also dephosphorylated by the Xanthomonas TTSS effector AvrAC (Feng et al., 2012).

Activation of the MAPK cascade is a common downstream event in plant resistance to pathogens, and the kinase activity of these MAPKs is modified by several bacterial effectors (Couto and Zipfel, 2016). HopF2 interacts with MKK5 to prevent its autophosphorylation (Wang et al., 2010c), and HopAl1 targets MPK3, MPK6 and MPK4 to dephosphorylate them (Zhang et al., 2007; Zhang et al., 2012).

AvrPto has many targets, including FLS2, EFR, SERK3 and Pto (Büttner, 2016). AvrPto binds to the kinase domain of FLS2 and EFR, thereby acting as a kinase inhibitor and blocking FLS2 and EFR auto-phosphorylation to compromise flg22/elf26-triggered immunity (Xiang et al., 2008). AvrPto also interacts with BAK1 (Shan et al., 2008), but 
whether AvrPto directly suppresses SERK3 kinase activity remains currently unknown. Surprisingly, the association between BRI1 and SERK3 is interrupted by AvrPto, but the phosphorylation of BRI1 and SERK3 upon BR treatment remains unaffected (Shan et al., 2008). Furthermore, AvrPto targets Pto, thereby suppressing Pto kinase activity (Xing et al., 2007). Taken together, it appears that AvrPto functions as a kinase inhibitor in suppressing the kinase activity of its interactor. In this study, we found that AvrPto targets both SOBIR1 and SERK3 (Fig. 1). Therefore, we anticipate that AvrPto might play a role in altering the phosphorylation status of SOBIR1 and/or SERK3 to suppress Cf-4/Avr4-triggered cell death (Fig. 6), without affecting Cf-4/S/SOBIR1/SISERK3a complex formation (Fig. 7).

\section{The requirement of kinase activity of targeted proteins for their interaction with effectors}

Targeting of host kinases by pathogen effectors is a common strategy to suppress MTI. In most cases, the kinase domain itself is targeted and kinase activity of the targeted proteins plays a role in the interaction. For example, AvrAC interacts with BIK1 to uridylylate the BIK1 phosphorylation site (Feng et al., 2012). Kinase activity of BIK1 is not required for the interaction, but it affects the uridylylation by AvrAC (Feng et al., 2012).

As mentioned above, AvrPtoB targets the kinase domain of SERK3, Pto, FLS2, CERK1 and Bti9 (Gimenez-lbanez et al., 2009; Göhre et al., 2008; Kim et al., 2002; Shan et al., 2008; Zeng et al., 2012). Kinase activity of SERK3 and Pto is required for the interaction with AvrPtoB (Cheng et al., 2011; Dong et al., 2009). However, whether kinase activity of FLS2, CERK1 and Bti9 is required for their interaction with AvrPtoB is unknown.

Kinase activity of Pto is required for its interaction with AvrPto. It has been shown that the threonine 204 mutant $\mathrm{Pto}^{\mathrm{T} 204 \mathrm{~N}}$, which has low kinase activity, only weakly interacts with AvrPto, whereas the kinase-inactive variant Pto ${ }^{526 \mathrm{D}}$ completely loses its interaction with the effector (Xing et al., 2007). Threonine 204 of Pto is highly conserved among the kinase domains of SERK3, SERK4, FLS2 and EFR (Shan et al., 2008). In SERK3, threonine 455 is equivalent to threonine 204 of Pto and a SERK3 ${ }^{\text {T455N }}$ mutant has reduced kinase activity (Lin et al., 2014). However, this substitution in SERK3 does not affect its interaction with AvrPto (Shan et al., 2008). It has been shown that Pto has evolved to compete for AvrPto interaction with FLS2 through its ATP-binding site (Xing et al., 2007), and has higher affinity for AvrPto than FLS2 (Xiang et al., 2008). AvrPto targets the ATP-binding site in the kinase domain of FLS2, and a mutation $(\mathrm{K} 898 \mathrm{H})$ in the ATP-binding site of FLS2, which is anticipated to have a lower kinase activity, 
abolishes its interaction with AvrPto (Xiang et al., 2008; Xing et al., 2007). Although it is unknown whether the kinase domain of SOBIR1 by itself is sufficient for interaction with AvrPto, kinase activity of SOBIR1 appears not to be required (Fig. 3). This observation reveals different requirements for the interaction of AvrPto with its targets, indicating that AvrPto utilizes different mechanisms to target and affect plant kinases involved in defence signalling.

\section{Materials and Methods}

\section{Plant materials and growth conditions}

Nicotiana benthamiana, Cf-4-transgenic N. benthamiana (Gabriëls et al., 2006) and N. tabacum were grown under $16 \mathrm{~h}$ of light at $25^{\circ} \mathrm{C}$ and $8 \mathrm{~h}$ of darkness at $21^{\circ} \mathrm{C}$ in climate chambers with a relative humidity of $75 \%$.

\section{Binary vectors for Agrobacterium infiltrations (agro-infiltrations)}

A fragment consisting of the AvrPto coding sequence in an entry vector was transferred to the destination vector pBIN-KS (for C-terminally tagging with eGFP) to generate AvrPto-eGFP, and pGWB14 (for C-terminally tagging with the HA epitope) to generate AvrPto-HA. SISERK3a-HA, SISOBIR1-HA, SISOBIR1-like-eGFP, SISOBIR1D473N_ eGFP, NbSOBIR1-eGFP, GUS-eGFP and GUS-HA were generated from entry vectors which have been described previously (Bi et al., 2016; Liebrand et al., 2013). SISERK3a-eGFP, SISOBIR1-eGFP, SISOBIR1-Myc, Cf-4-eGFP, AtSOBIR1-eGFP,

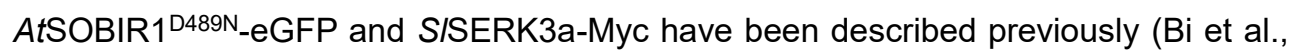
2016; Liebrand et al., 2013; Postma et al., 2016). Silencing suppressor P19, which was included in all co-IP experiments, has been described previously (Voinnet et al., 2015).

Binary vectors were transformed to Agrobacterium tumefaciens strain C58C1, carrying helper plasmid pCH32. Infiltration of Agrobacterium into plant leaves was performed as described at an OD600 of 1, unless indicated otherwise (van der Hoorn et al., 2000).

\section{Co-immunoprecipitations (co-IPs) and immunoblotting}

Co-IPs were performed as described previously (Liebrand et al., 2012). The following antibodies were used: anti-p42/p44-ERK (New England Biolabs), in combination with goat anti-rabbit (Sigma) as a secondary antibody, anti-GFP (Miltenyi Biotec $\mathrm{GmbH}$ ), anti-HA (clone 3F10; Roche Applied Science), anti-cMyc (9E10; Santa Cruz Biotechnology), with sheep anti-mouse (Amersham) as a secondary antibody. 
AvrPto targets SOBIR1 to suppress Cf-4 signalling

\section{Acknowledgments}

T.W.H. Liebrand and R.R. Bye are acknowledged for initiating experiments on AtSOBIR1-induced constitutive cell death in N. tabacum. L. Deurhof is acknowledged for technical support. J. Wu and G. Bi are supported by the China Scholarship Council (CSC). A.M. van der Burgh is supported by the Netherlands Organization for Scientific Research (NWO), division Earth and Life Sciences (ALW). 


\section{Chapter 3}

\section{Supplementary information}
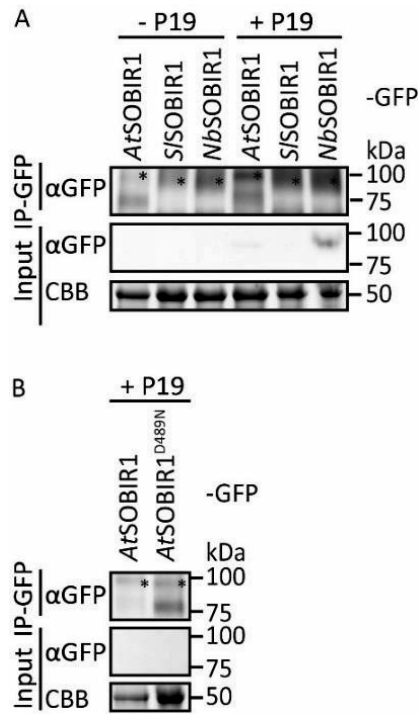

Figure S1. All SOBIR1 variants accumulate in planta. (A) Accumulation levels of At-, SI- and NbSOBIR1 without and with silencing suppressor P19. C-terminally eGFP-tagged SOBIR1 variants were transiently expressed in $N$. benthamiana with an OD of 1 . After 2 days, total proteins were extracted and subjected to immunoprecipitation using GFP_TrapA affinity beads. Immunoprecipitated proteins (IP) were subjected to SDS-PAGE and blotted to PVDF membrane. Blots were probed with aGFP antibodies to detect immunoprecipitated eGFP-tagged proteins (upper panels). Total protein staining indicates the amount of protein that was loaded (lower panel). Bands with the expected size of full-length AtSOBIR1GFP, SISOBIR1-GFP and NbSOBIR1-GFP are indicated with an asterisk. (B) Accumulation levels of

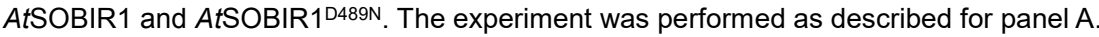




\section{Chapter 4}

\section{An EFR-Cf-9 chimera confers enhanced resistance to bacterial pathogens by SOBIR1- and BAK1-dependent recognition of elf18}

Jinbin $\mathrm{Wu}^{1 \#}$, Ida-Barbara Reca ${ }^{2 \#}$, Francesco Spinelli ${ }^{3}$, Damiano Lironi ${ }^{3}$, Giulia De Lorenzo ${ }^{3}$, Palmiro Poltronieri ${ }^{2}$, Felice Cervone ${ }^{3}$, Matthieu H.A.J. Joosten ${ }^{1}$, Simone Ferrari ${ }^{3^{*}}$ and Alexandre Brutus ${ }^{4}$

'Laboratory of Phytopathology, Wageningen University \& Research, Droevendaalsesteeg 1, 6708 PB Wageningen, The Netherlands.

${ }^{2}$ CNR-ISPA, via Provinciale Lecce-Monteroni, 73100 Lecce, Italy.

${ }^{3}$ Department of Biology and Biotechnology "Charles Darwin", Sapienza University of Rome, 00185 Rome, Italy.

${ }^{4}$ DOE Plant Research Laboratory, Michigan State University, East Lansing, MI 48824, USA

"Equal contribution

${ }^{*}$ Corresponding author

A slightly modified version has been published in: Molecular Plant Pathology (2019) 20: 751764. 


\section{Chapter 4}

\section{Abstract}

The transfer of well-studied native and chimeric pattern recognition receptors (PRRs) to susceptible plants is a proven strategy to improve host resistance. In most cases, the ectodomain determines PRR recognition specificity, while the endodomain determines the intensity of the immune response. Here we report the generation and characterization of the chimeric receptor EFR-Cf-9, which carries the ectodomain of the Arabidopsis thaliana EF-Tu receptor (EFR) and the endodomain of the tomato Cf-9 resistance protein. Both transient and stable expression of EFR-Cf-9 triggered a strong hypersensitive response (HR) upon elf18 treatment in tobacco. Co-immunoprecipitation and virus-induced gene silencing studies showed that EFR-Cf-9 constitutively interacts with the regulatory RLK SUPPRESSOR OF BIR1-1 (SOBIR1) and requires both SOBIR1 and kinase-active BRI1-ASSOCIATED KINASE 1 (BAK1) for its function. Transgenic plants expressing EFR-Cf-9 were more resistant to the (hemi)biotrophic bacterial pathogens Pseudomonas amygdali pv. tabaci (Pta) 11528 and P. syringae pv. tomato DC3000. These transgenic plants mounted an HR in response to high doses of Pta 11528 and of the necrotrophic bacterium Pectobacterium carotovorum. Taken together, these data indicate that the EFR-Cf-9 chimera is a valuable tool for both investigating the molecular mechanisms responsible for the activation of defence responses by PRRs, and for potential biotechnological use to improve crop disease resistance. 


\section{Introduction}

Plants have evolved an innate immune system that relies on the recognition of potential pathogens by a defined pool of membrane and cytosolic receptors. The first layer of plant immunity comprises plasma membrane pattern recognition receptors (PRRs) that recognize microbe-associated molecular patterns (MAMPs), thereby mounting MAMPtriggered immunity (MTI) (Couto and Zipfel, 2016; Dangl et al., 2013; Dodds and Rathjen, 2010; Jones and Dangl, 2006). Successful pathogens often produce effector proteins capable of suppressing MTI and preventing an effective immune response (Dodds and Rathjen, 2010; Jones and Dangl, 2006). As a countermeasure, plants have evolved pathogen strain-specific immune receptors, so-called resistance $(R)$ proteins, which are capable of recognizing particular effectors and subsequently activate effector-triggered immunity (ETI) (Dodds and Rathjen, 2010; Jones and Dangl, 2006). Although leading to outputs that are qualitatively similar to those of $\mathrm{MTI}, \mathrm{ETI}$ is generally stronger and faster, and often includes a rapid programmed cell death (PCD) of the host cells at the site of infection, referred to as hypersensitive response (HR) (Dodds and Rathjen, 2010; Jones and Dangl, 2006).

Cell surface PRRs are either receptor-like kinases (RLKs) or receptor-like proteins (RLPs), of which the latter lack an intracellular kinase domain (Boutrot and Zipfel, 2017; Zipfel, 2014). Both types of receptors possess an ectodomain for ligand recognition that in many cases is mainly composed of leucine-rich repeats (LRRs) (Boutrot and Zipfel, 2017; Zipfel, 2014). In tomato (Solanum lycopersicum, SI), the well-studied RLP Cf-4 triggers a strong HR-associated immunity against the biotrophic pathogenic fungus Cladosporium fulvum secreting the effector Avr4 (Joosten et al., 1997; Thomas et al., 1997). Cf-4 shares identical transmembrane (TM) and cytoplasmic domains with Cf-9 (Thomas et al., 1997), which confers resistance to $C$. fulvum secreting the effector Avr9 (Jones et al., 1994; van den Ackerveken et al., 1992; van Kan et al., 1991). The regulatory RLK SUPPRESSOR OF BIR1-1/EVERSHED (SOBIR1/EVR, hereafter referred to as SOBIR1) is required for $\mathrm{Cf}-4$-mediated resistance to $C$. fulvum (Liebrand et al., 2013) and employs its TM domain to constitutively interact with Cf-4 (Bi et al., 2016). Increasing evidence suggests that SOBIR1 constitutively interacts with several RLPs, providing the RLP/SOBIR1 complex with a kinase domain to trigger downstream signalling pathways (Albert et al., 2015; Böhm et al., 2014a; Domazakis et al., 2018; Gust and Felix, 2014; Hegenauer et al., 2016; Liebrand et al., 2013; Liebrand et al., 2014; Ma and Borhan, 2015; Zhang et al., 2014; Zhang et al., 2013a). An additional regulatory $\mathrm{RLK}, \mathrm{BAK} 1$, the orthologue of the Arabidopsis thaliana (At) BRIASSOCIATED RECEPTOR KINASE 1/SOMATIC EMBRYOGENESIS RECEPTOR KINASE 3 (BAK1/SERK3, hereafter referred to as AtBAK1), is recruited to the Cf- 
4/SOBIR1 complex after perception of Avr4 by Cf-4 and is required for Cf-4-mediated resistance (Postma et al., 2016).

Elf18, an 18-amino-acid N-terminal epitope of the bacterial elongation factor Tu (EFTu) (Kunze et al., 2004), is recognized by the Arabidopsis LRR-RLK EF-Tu receptor (EFR) and triggers MTI (Zipfel et al., 2006). The 'prototypical' elf18 peptide from Escherichia coli (MSKEKFERTKPHVNVGTI; elf18D, hereafter referred to as elf18) and those from Erwinia amylovora and E. chrysanthemi are identical and show full activity, whereas elf18 peptides with slightly varying sequences, as found in additional bacteria, show different activity (Kunze et al., 2004). For example, elf18C from Agrobacterium strains, and elf18F, shared by different Pseudomonas species, including $P$. amygdali pv. tabaci (Pta) 11528, are fully active, whereas elf18B and elf18G, found in Xanthomonas campestris pv. campestris (Xcc) B100 and $P$. syringae pv. tomato (Pst) DC3000, respectively, trigger a weaker response (Lacombe et al., 2010). Besides elf18, a widely studied MAMP is flg22, an epitope of the bacterial flagellin that is recognized by the LRR-RLK FLAGELLIN-SENSING 2 (FLS2) (Felix et al., 1999; Gómez-Gómez and Boller, 2000; Zipfel et al., 2004).

Interfamily transfer of PRRs is an effective strategy for improving resistance of plants to a wide range of infectious microbes (Dangl et al., 2013). For example, EFR confers broad-spectrum resistance to bacterial pathogens in tomato and Nicotiana benthamiana $(\mathrm{Nb})$ (Lacombe et al., 2010), and also in the monocot rice (Schwessinger et al., 2015). The rice resistance protein Xa21, which confers resistance to Pst DC3000 upon transfer to Arabidopsis (Holton et al., 2015), has also been transferred to crops like citrus, tomato and banana to confer resistance to Xanthomonas species (Afroz et al., 2011; Mendes et al., 2010; Tripathi et al., 2014). Strategies based on stacking multiple resistance genes are also pursued to avoid that the $R$ gene-mediated resistance might be overcome by pathogens. For example, transgenic potato (Solanum tuberosum) plants expressing three $R$ genes against Phytophthora infestans show robust resistance (Chen and Ow, 2017; Halpin, 2005; Zhu et al., 2012).

Recent research provides evidence that engineering novel recombinant PRRs by domain swapping to obtain chimeric receptors that combine useful features of different PRRs is a promising option to breed for durable and wide spectrum resistance (Boutrot and Zipfel, 2017; De Lorenzo et al., 2011). Significant progress has been achieved, leading to the indication that the ectodomain of a chimeric receptor retains the ligand perception ability, while the endodomain maintains the output intensity (Albert et al., 2010; Brutus et al., 2010; He et al., 2000; Holton et al., 2015; Kishimoto et al., 2010; Kouzai et al., 2013; Schwessinger et al., 2015; Thomas et al., 2017). For instance, we 
have previously reported that the chimera FLS2-EFR, carrying the ectodomain of FLS2 and the kinase domain of EFR, recognizes flg22 and induces an EFR-like response in $N$. tabacum (Nt, tobacco), which lacks an endogenous EFR receptor (Lacombe et al., 2010). This response typically results in a higher ethylene production than that induced by FLS2 upon perception of flg22 (Brutus et al., 2010). WALL-ASSOCIATED KINASE 1 (WAK1) is a receptor of oligogalacturonides (OGs) and triggers defence responses upon perception of this so-called damage-associated molecular pattern (DAMP). Upon OG treatment, a chimera comprising the ectodomain of WAK1 and the EFR kinase domain triggers an EFR-like response (Brutus et al., 2010). On the other hand, a reciprocal EFR-WAK1 chimera recognizes elf18 to induce a stronger OG/WAK1-like oxidative burst response than that triggered by EFR upon perception of elf18 (Brutus et al., 2010). However, the downstream signalling components employed by the transferred PRRs and chimeras are largely unknown, and the signalling pathways that are triggered in the plant from which the PRR originates and those in the recipient plant might not be conserved.

In this work, we aimed to obtain a strong MAMP-dependent resistance response in tobacco, by exploiting the EFR-mediated recognition of the broad-spectrum bacterial MAMP elf18 and the ability of Cf-9 to trigger a strong HR upon its activation (Jones et al., 1994; van den Ackerveken et al., 1992; van Kan et al., 1991; Zipfel et al., 2006). Tobacco SR1 plants transiently expressing the chimera EFR-Cf-9, harbouring the ectodomain of EFR and the TM and cytoplasmic domain of Cf-9 (hereafter referred to as the endodomain), indeed mount a strong elf18-triggered HR. We show that chimeric EFR-Cf-9 protein constitutively interacts with SOBIR1 and requires both SOBIR1 and BAK1 for functionality. Transgenic tobacco plants expressing EFR-Cf-9 activate an HR upon elf18 treatment and show enhanced resistance to the (hemi)biotrophic bacterial pathogens Pta 11528 and Pst DC3000.

\section{Results}

\section{EFR-Cf-9 recognizes elf18C to trigger a strong HR in tobacco plants}

We have previously reported that the ectodomain of EFR (Lys649 $9_{\mathrm{EFR}}$ ) is functional in an EFR-WAK1 chimera and perceives elf18, while the ectodomain of FLS2 (Arg806 ${ }_{\mathrm{FLS} 2}$ ) is functional in an FLS2-EFR chimera (eJMC in the original article) and perceives flg22 (Brutus et al., 2010). Here, we fused the ectodomains of EFR or FLS2 to the endodomain (Trp811cf-9) of Cf-9 (Jones et al., 1994), to obtain EFR-Cf-9 and FLS2-Cf9 chimeras, respectively (Fig. 1). Both chimeras were in turn fused to eGFP and transiently expressed by agro-infiltration in leaves of tobacco plants stably overexpressing Avr9 (Hammond-Kosack et al., 1998) or transiently co-expressing Avr9. 
EFR

\begin{tabular}{|c|c|c|c|c|c|}
\hline $\mathrm{SP}$ & LRR & eJM & $\mathrm{TM}$ & iJM & $\mathrm{K}$ \\
\hline \multicolumn{2}{|c|}{ EFR-Cf-9 } & \multicolumn{3}{|c|}{ Lys649 ${ }_{\text {EFr }}$-Trp811 $^{\text {Cf-9 }}$} & \\
\hline $\mathrm{SP}$ & LRR & eJM & TM & iJM & \\
\hline \multicolumn{5}{|l|}{ Cf-9 } & \\
\hline SP & LRR & eJM & $\mathrm{TM}$ & iJM & \\
\hline \multicolumn{2}{|c|}{ FLS2-Cf-9 } & \multicolumn{3}{|c|}{$\operatorname{Arg} 806_{\mathrm{FLS} 2}-\mathrm{Tr}{ }^{-11_{\mathrm{Cf}-9}}$} & \\
\hline SP & LRR & eJM & TM & iJM & \\
\hline \multicolumn{6}{|c|}{ FLS2 } \\
\hline $\mathrm{SP}$ & LRR & eJM & TM & iJM & $\mathrm{K}$ \\
\hline
\end{tabular}

Figure 1. Overview of the structure of the chimeric receptors. The coding regions of EFR, Cf-9 and FLS2 are indicated in dark grey, white and light grey, respectively. The arrows indicate the junction between the EFR or FLS2 ectodomain and the Cf-9 transmembrane (TM) domain, with the corresponding residues included in the chimeras. SP, signal peptide; LRR, leucine-rich repeat; eJM, external juxtamembrane; iJM, internal juxtamembrane; $\mathrm{K}$, kinase.

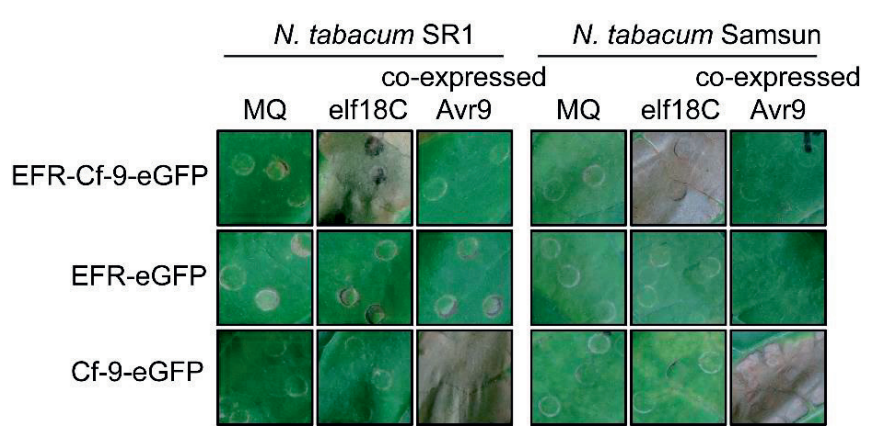

Figure 2. EFR-Cf-9 recognizes elf18C and triggers an HR. EFR-Cf-9, EFR and Cf-9, all fused to eGFP, were transiently expressed in WT $N$. tabacum SR1 (left) and $N$. tabacum Samsun (right) plants $(n=4)$. Two days after agro-infiltration, leaves were treated with Milli-Q water (MQ) or $100 \mathrm{nM}$ elf18C and pictures were taken after two days. As a positive control, eGFP-tagged EFR-Cf-9, EFR and Cf-9 were also co-infiltrated with an Agrobacterium strain driving expression of Avr9, and pictures were taken at 12 days post-infiltration (dpi). At these time points, all leaves agro-infiltrated with EFR-Cf-9 and subsequently infiltrated with el18C, and all leaves co-expressing Cf-9 and Avr9 showed necrosis of at least half of the infiltrated area, whereas none of the other leaves showed any HR-like symptoms. Experiments were repeated three times with similar results. Representative images are shown.

In parallel, EFR-eGFP, FLS2-eGFP and Cf-9-eGFP fusions were agro-infiltrated as controls. Leaf areas transiently expressing eGFP-tagged EFR, EFR-Cf-9, FLS2 and FLS2-Cf-9 did not exhibit any symptom, while leaves co-expressing Cf-9-eGFP and Avr9, as expected, displayed a clear HR (Fig. S1 and S2). These results indicate that the chimeras are not responsive to Avr9 and do not per se induce HR-like symptoms. Next, whether EFR-Cf-9 and FLS2-Cf-9 are functional and trigger a ligand-dependent HR-like response was tested. Upon infiltration with $100 \mathrm{nM}$ elf18C, leaf areas transiently expressing EFR-Cf-9-eGFP showed a strong HR, similar to the Cf-9- 
eGFP/Avr9 control, in two different tobacco cultivars (SR1 and Samsun) (Fig. 2). In contrast, leaf areas transiently expressing FLS2-Cf-9-eGFP did not show visible symptoms upon treatment with $100 \mu \mathrm{M}$ flg22 (Fig. S2), suggesting that FLS2-Cf-9eGFP is not functional in tobacco.

Together, these data show that EFR-Cf-9 recognizes elf18C to trigger a strong Cf9/Avr9-like HR, indicating that while the ectodomain of EFR retains the ability to perceive the elf $18 \mathrm{C}$ peptide, its fusion to the endodomain of $\mathrm{Cf}-9$ results in an output similar to that of the native Cf-9.
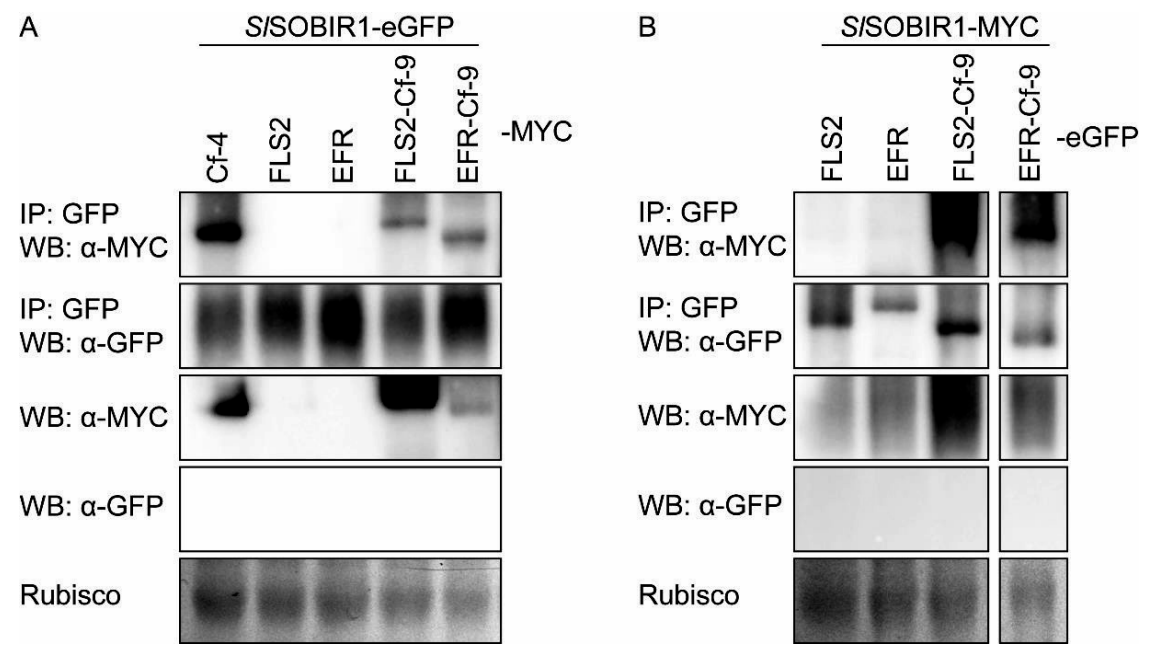

Figure 3. Both FLS2-Cf-9 and EFR-Cf-9 interact with SOBIR1. (A) eGFP-tagged tomato (SI) SOBIR1 was transiently co-expressed with Myc-tagged Cf-4, FLS2, EFR, FLS2-Cf-9 and EFR-Cf-9. (B) Myctagged SISOBIR1 was transiently co-expressed with eGFP-tagged FLS2, EFR, FLS2-Cf-9 and EFR-Cf9. Agrobacteria driving expression of the various constructs were infiltrated at a final $O D_{600}$ of 1 . After two days, proteins were extracted and immunoprecipitated (IP) using GFP-Trap beads. Proteins were detected by immunoblot (WB) using $\alpha$-GFP and $\alpha-M y c$ antibodies. Equal loading is indicated by Rubisco band. Experiments were repeated three times and representative images are shown.

\section{EFR-Cf-9 functionality requires SOBIR1 and BAK1}

It has been reported that the TM domain of SOBIR1 is required for its interaction with Cf-4 (Bi et al., 2016) and that the regulatory RLKs SOBIR1 and BAK1 are both required for the function of Cf-4 (Liebrand et al., 2013; Postma et al., 2016). Cf-9 shares an identical endodomain with Cf-4 (Thomas et al., 1997) and also interacts with SOBIR1 
(Liebrand et al., 2013). We therefore investigated whether EFR-Cf-9 also interacts with tomato SOBIR1 (henceforth indicated as SISOBIR1). EFR-Cf-9-Myc was generated and co-expressed with SOBIR1-eGFP in N. benthamiana. In parallel, FLS2-Cf-9 (as the non-functionality of this chimera might be due to a possible lack of interaction with SOBIR1), Cf-4-eGFP, FLS2-eGFP and EFR-eGFP were agro-infiltrated as controls. Immunoprecipitation (IP) of SISOBIR1 using GFP-Trap beads demonstrated that Cf-4 was co-precipitated with SISOBIR1 (Fig. 3A), which is consistent with our previous finding (Liebrand et al., 2013). In addition, FLS2-Cf-9 and EFR-Cf-9 were also copurified with SISOBIR1 (Fig. 3A), indicating that, similar to the Cf proteins, both chimeras constitutively interact with SISOBIR1. In reverse co-immunoprecipitation assays (co-IPs), IP of both FLS2-Cf-9-eGFP and EFR-Cf-9-eGFP resulted in the coprecipitation of SISOBIR1-Myc (Fig. 3B). In both cases, the FLS2 and EFR wild-type proteins did not interact with SISOBIR1 (Fig. 3A and B), confirming the specificity of SOBIR1 for RLPs (Gust and Felix, 2014; Liebrand et al., 2013; Liebrand et al., 2014).

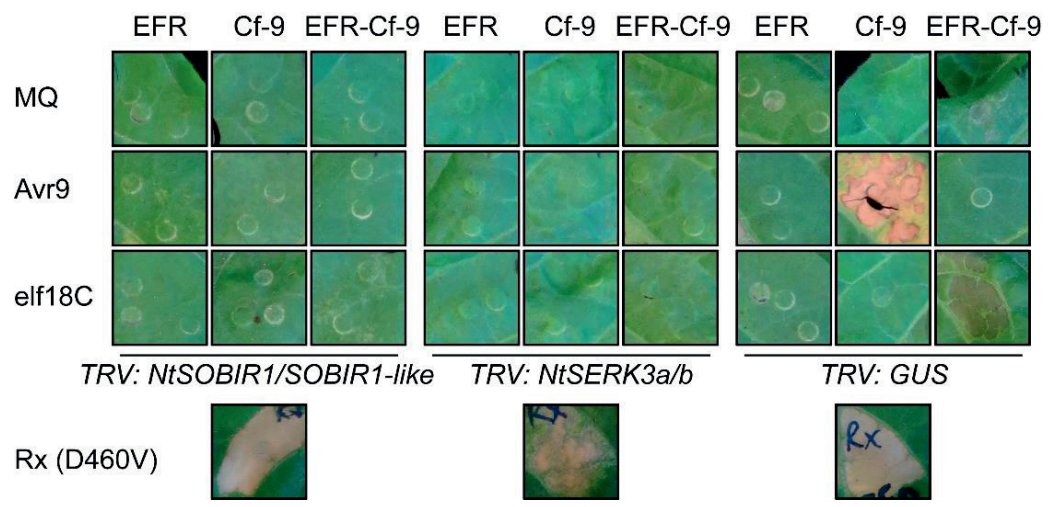

Figure 4. SOBIR1 and BAK1 are required for the EFR-Cf-9-dependent elf18C-triggered HR. eGFPtagged EFR, Cf-9 and EFR-Cf-9 were either transiently expressed with Avr9, or expressed alone, in $N$. tabacum Samsun plants silenced for NtSOBIR1/SOBIR1-like, NtSERK3a/b or GUS $(\mathrm{n}=4)$. Agrobacteria driving expression of the constructs were infiltrated at a final $\mathrm{OD}_{600}$ of 1 , except for the positive control $\mathrm{Rx}(\mathrm{D} 460 \mathrm{~V})$, which was infiltrated at an $\mathrm{OD}_{600}$ of 0.1 . At $2 \mathrm{dpi}$, leaves were treated with Milli-Q water (MQ) or $100 \mathrm{nM}$ elf18C. Pictures were taken at two days after treatment with elf18C or, in the case of Cf-9-Avr9 interaction, at 12 days after co-infiltration of Cf-9 and Avr9. Experiments were repeated three times with similar results and representative images are shown.

Next, we investigated whether EFR-Cf-9 requires SOBIR1 and/or BAK1 to trigger an HR upon perception of elf18C. Tobacco Samsun plants were used for silencing NtSOBIR1/SOBIR1-like and NtSERK3a/b, orthologues of SOBIR1 and BAK1, respectively (Heese et al., 2007; Liebrand et al., 2013). eGFP-tagged EFR, Cf-9 and 
EFR-Cf-9 were either transiently co-expressed with Avr9, or expressed alone in the silenced plants followed by treatment with Milli-Q (MQ) water or $100 \mathrm{nM}$ elf18C. Consistent with earlier studies (Liebrand et al., 2013; Postma et al., 2016), silencing of NtSOBIR1/SOBIR1-like and of NtSERK3a/b strongly suppressed the HR triggered by the Cf-9/Avr9 combination, but did not affect the ability of the plant to mount PCD triggered by $\mathrm{Rx}$ (D460V) (Fig. 4), which is a constitutively active form of the $R x$ gene, providing resistance against Potato Virus X (PVX) (Bendahmane et al., 2002). The EFR-Cf-9-dependent elf18C-triggered HR was also severely compromised in the silenced plants, indicating that both NtSOBIR1/SOBIR1-like and NtSERK3a/b are required for EFR-Cf-9 functionality (Fig. 4). Control plants, which were inoculated with Tobacco Rattle Virus carrying the $\beta$-glucuronidase (GUS) gene (TRV-GUS), did not show any defect in the EFR-Cf-9-dependent HR (Fig. 4).

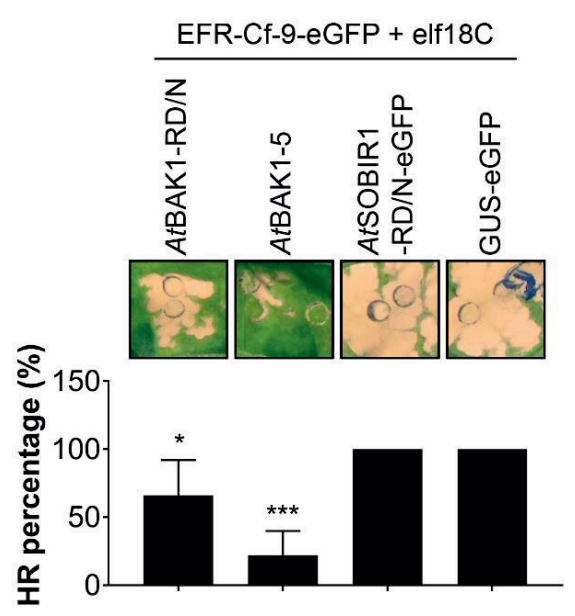

Figure 5. EFR-Cf-9- dependent elf18C-triggered HR requires kinase activity of BAK1. EFR-Cf-9-eGFP was transiently co-expressed with the kinase-dead mutant AtBAK1-RD/N, the hypoactive mutant AtBAK15, the kinase-dead mutant AtSOBIR1-RD/N-eGFP and GUS-eGFP at a final OD 600 of $1(n=5)$ in $N$. tabacum Samsun plants. At $2 \mathrm{dpi}$, leaves were treated with 100 $\mathrm{nM}$ elf18C, and pictures were taken at 2 days after treatment. Bars in the bottom panel show the average percentage of the infiltrated area showing necrosis \pm $\mathrm{SD}(\mathrm{n}=5)$. The asterisks indicate significant difference with GUS-GFP, determined by Student's $t$ test ${ }^{*} P<$ 0.05 , $\left.{ }^{\star \star *} P<0.001\right)$. Experiments were repeated three times with similar results, and representative images are shown (top panel).

Kinase activity of both SOBIR1 and BAK1 is required for the function of Cf-4 (Liebrand et al., 2013; Postma et al., 2016). The AtBAK1-5 mutant contains a point mutation in its kinase domain, which causes this protein to have a slightly lower kinase activity than BAK1 itself (Schwessinger et al., 2011). In addition, AtBAK1-5 has a dominant-negative effect on the BAK1-dependent immune response mediated by FLS2 and EFR (Schwessinger et al., 2011). In order to study whether kinase activity of BAK1 is also required for EFR-Cf-9 functionality, and whether the kinase-dead variant AtSOBIR1$\mathrm{RD} / \mathrm{N}$ (Liebrand et al., 2013) displays a dominant-negative effect similar to the hypoactive kinase AtBAK1-5 and kinase-dead AtBAK1-RD/N mutants (Schwessinger et al., 2011), EFR-Cf-9-eGFP was transiently co-expressed with AtBAK1-RD/N, AtBAK1-5, AtSOBIR1-RD/N-eGFP and GUS-eGFP, followed by treatment with $100 \mathrm{nM}$ 
elf18C. The EFR-Cf-9-dependent elf18C-triggered HR was not affected by GUS-eGFP, but was reduced by AtBAK1-RD/N and even more compromised by AtBAK1-5 (Fig. 5), indicating that both BAK1 mutants have a dominant-negative effect on the EFR-Cf-9triggered HR, possibly because the overexpressed BAK1 mutants compete away the endogenous NtSERK3a/b from the activated EFR-Cf-9-containing signalling complex. We conclude that the EFR-Cf-9-dependent elf18C-triggered HR depends on the kinase activity of BAK1. Unlike AtBAK1-RD/N and AtBAK1-5, AtSOBIR1-RD/N-eGFP did not affect the HR (Fig. 5). Considering that RLPs constitutively interact with SOBIR1, whereas the recruitment of BAK1 to the signalling complex is ligand-dependent, it is possible that transiently expressed AtSOBIR1-RD/N-eGFP may fail to displace the endogenous NtSOBIR1/SOBIR1-like protein that is bound to EFR-Cf-9.

Taken together, these data indicate that both SOBIR1 and BAK1 are required for the EFR-Cf-9-dependent elf18C-triggered HR, and that BAK1 has to be kinase-active.

\section{EFR-Cf-9-transgenic tobacco plants are differentially responsive to elf18 variants}

An untagged version of EFR-Cf-9 was stably expressed in tobacco SR1 plants. Fourteen primary transformants were obtained, of which five had detectable expression of EFR-Cf-9 (Fig. 6A) and were therefore propagated. Two independent lines ( $\mathrm{K} 1 \mathrm{~A}$ and $\mathrm{K} 5 \mathrm{~A}$ ) showed a $3: 1$ segregation ratio for kanamycin resistance, suggesting that they carried an insertion in a single locus, and were selected for further characterization. Transgenic plants were morphologically identical to the parental plants (Fig. 6B). To verify that the chimera was properly expressed and localized, trypsin digestion of microsomal leaf proteins from WT and K1A plants was performed, followed by liquid chromatography-tandem mass spectrometry (LC-MS/MS) analysis. Six peptides corresponding to the ectodomain of EFR (coverage of 15.49; score of 16.31) were found only in extracts from the transgenic plants (Fig. S3), confirming that EFR-Cf-9 is expressed and is likely membrane-localized. No peptide corresponding to the chimera was found in WT extracts.

Subsequently, the functionality of EFR-Cf-9 in K1A and K5A plants was assessed. Only leaves from both transgenic lines mounted an HR upon elf18C treatment, at concentrations ranging from $1 \mathrm{nM}$ to $10 \mu \mathrm{M}$ (Fig. 6C). Responsiveness to different elf18 variants was also tested in line $\mathrm{K} 1 \mathrm{~A}$, revealing that an $\mathrm{HR}$ was triggered also by elf18B and elf18G, although to a lesser extent when compared with elf18C (Fig. S4). This suggests that, although the EFR ectodomain was fused to the endodomain of Cf-9, the structure of the ligand still determines the output. To obtain a more quantitative evaluation of the response mediated by EFR-Cf- 9 , and to assess whether the presence 
A

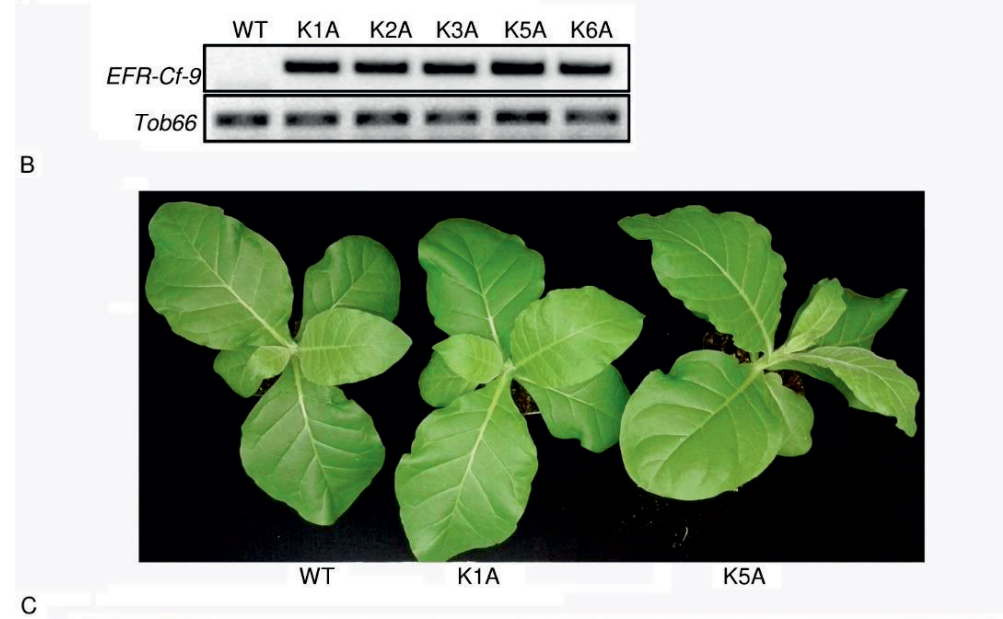

C

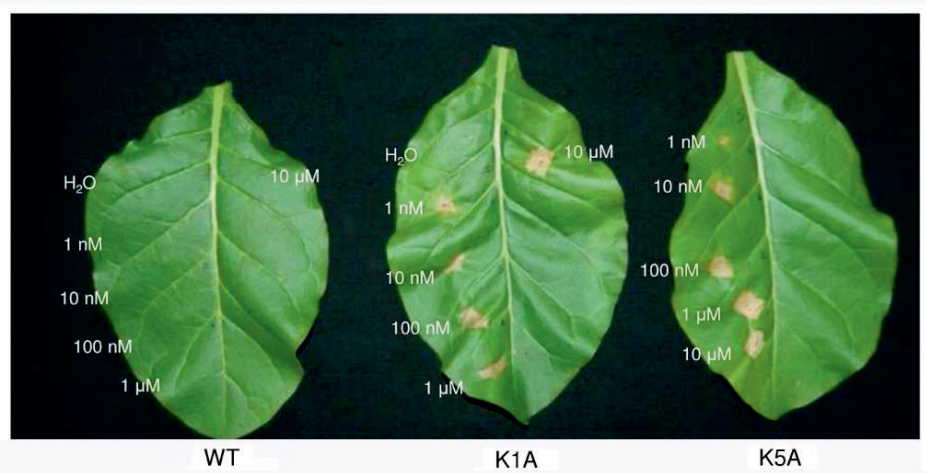

Figure 6. EFR-Cf-9-transgenic plants do not show morphological changes and recognize elf18C to trigger HR. (A) Expression of EFR-Cf-9 in leaves of WT plants and five primary transformants (K1A, $\mathrm{K} 2 \mathrm{~A}, \mathrm{~K} 3 \mathrm{~A}, \mathrm{~K} 5 \mathrm{~A}$ and $\mathrm{K} 6 \mathrm{~A}$ ) was evaluated by RT-PCR, using Tob66 as a control. (B) Picture of representative four-week-old WT and transgenic K1A and K5A plants. (C) Fully expanded leaves of fourweek-old WT and EFR-Cf-9-transgenic plants (K1A and K5A) $(n>3)$ were infiltrated with elf18C at the indicated concentrations. Pictures were taken at $5 \mathrm{dpi}$. All leaves from transgenic plants showed necrosis of at least $50 \%$ of the area infiltrated with elf $18 \mathrm{C}$, whereas those infiltrated with water and all WT leaves failed to display HR-like symptoms. Experiments were repeated three times and representative images are shown.

of EFR-Cf-9 might cause an elevated basal defence response, the expression of the marker genes Avr9/Cf-9 RAPIDLY ELICITED-132 (ACRE-132) (Durrant et al., 2000) and HAIRPIN INDUCED 1 (HIN1) (Gopalan et al., 1996) was analysed. Transcript levels of both genes were similar in $\mathrm{MQ}$ water-treated WT and K1A plants, indicating that EFR-Cf-9 does not affect basal defence (Fig. S5). In addition, as expected, WT plants did not respond to any of the elf18 variants tested, whereas they showed a 
significantly increased expression of ACRE-132 and HIN1 after flg22 elicitation (Fig. S5). In contrast, treatment of K1A plants with elf18C induced a significant increase in transcript levels for ACRE-132 and HIN1, which were even higher than in plants treated with flg22 (Fig. S5). Moreover, treatment with elf18B and elf18G also resulted in increased transcript levels for $A C R E-132$ and HIN1, but to a lesser extent than for elf18C (Fig. S5), which supports our finding that elf18C triggers a stronger HR than the elf18 variants (Fig. S4). On the other hand, WT and K1A plants displayed similar expression levels of both genes in response to flg22 (Fig. S5), indicating that EFR-Cf9 does not affect the endogenous NtFLS2-mediated response to flg22.

Together, these data indicate that transgenic tobacco plants expressing EFR-Cf-9 are not altered in their basal defence and in their responsiveness to flg22. Moreover, the EFR-Cf-9-transgenic plants respond more efficiently to elf18C than to the different elf18 variants.

\section{Transgenic tobacco plants expressing EFR-Cf-9 show enhanced resistance to (hemi)biotrophic bacterial pathogens}

To study whether the EFR-Cf-9-mediated immune response eventually leads to resistance against bacterial pathogens, WT and transgenic K1A and K5A plants were inoculated with the (hemi)biotrophic bacterial pathogens Pta 11528 and Pst DC3000 at a dose of $\mathrm{OD}_{600}=0.002$ (corresponding to $10^{4} \mathrm{CFU} / \mathrm{mL}$ ). Compared to WT, a clear reduction in bacterial colonization was found in both transgenic lines inoculated with Pta 11528 and, to an even greater extent, Pst DC3000 (Fig. 7A and B). To verify that EFR-Cf-9 indeed triggers an HR not only in response to the purified elf18 peptides, but also to bacterial infection, different doses $\left(\mathrm{OD}_{600}=0.002,0.02\right.$ and 0.2$)$ of Pta 11528 were inoculated on WT, K1A and K5A plants. Within 48h, leaf sectors of the transgenic plants inoculated with the two higher doses of bacteria $\left(\mathrm{OD}_{600}=0.02\right.$ and 0.2$)$ displayed HR-like symptoms, whereas WT plants did not show any symptoms with all doses of bacteria (Fig. 8A).

We also tested the susceptibility of these transgenic plants to the necrotrophic bacterium Pectobacterium carotovorum subsp. carotovorum strain DSMZ 30169, which is the causal agent of bacterial soft rot (Toth et al., 2003). It was reported that inoculation with $P$. carotovorum is able to induce an EFR-dependent response in Arabidopsis, indicating the presence of an elf18-like MAMP (Lacombe et al., 2010). Leaves inoculated with $P$. carotovorum at an OD600 of 0.02 (corresponding to about $10^{5}$ $\mathrm{CFU} / \mathrm{mL}$ ) showed a 10 -fold increase in bacterial count at $24 \mathrm{~h}$, with no significant differences between WT and the transgenic lines (Fig. 7C). Notably, HR-like symptoms were also observed in $\mathrm{K} 1 \mathrm{~A}$ and $\mathrm{K} 5 \mathrm{~A}$, but not in WT plants after inoculation with this 
A

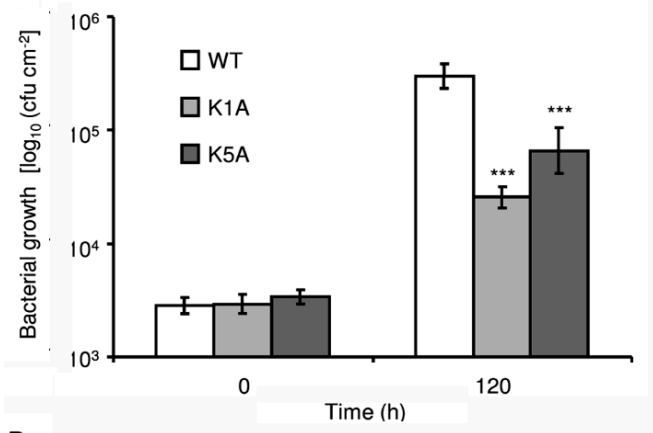

B

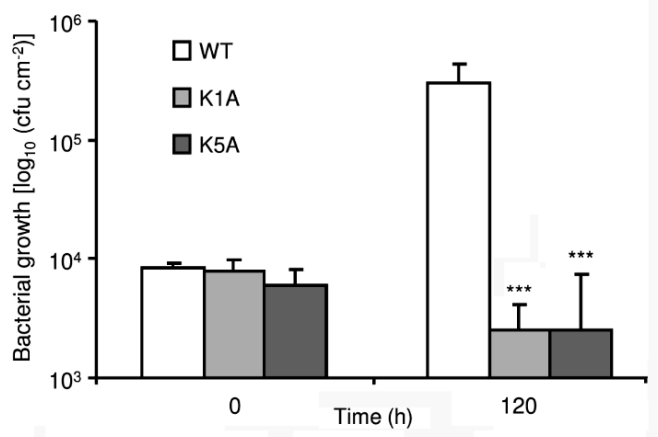

C

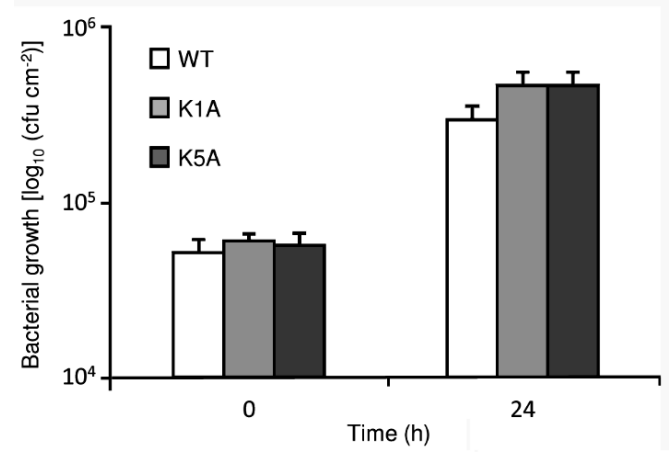

Figure 7. EFR-Cf-9-transgenic plants show altered resistance to bacterial pathogens. Fully expanded leaves of four-week-old untransformed plants (WT) and EFR-Cf-9transgenic plants $(\mathrm{K} 1 \mathrm{~A}$ and $\mathrm{K} 5 \mathrm{~A})$ were inoculated with Pseudomonas amygdali pv. tabaci (Pta) 11528 (A) or P. syringae pv. tomato DC3000 (B) at an $\mathrm{OD}_{600}$ of 0.002 (approximately $10^{4} \mathrm{CFU} / \mathrm{mL}$ ), or $P$. carotovorum (C) at $\mathrm{OD}_{600}$ of 0.02 (approximately $10^{5} \mathrm{CFU} / \mathrm{mL}$ ). Bacterial colonization was determined at the indicated time points ( $n>3$ for each time point). Asterisks indicate statistically significant differences between WT and transgenic lines, according to Student's $t$-test $\left({ }^{* *} P<0.0001\right)$.

bacterium (Fig. 8B). These observations suggest that EF-Tu of $P$. carotovorum is recognized by EFR-Cf- 9 and triggers an HR that is not able to promote colonization by this pathogen.

The fungal pathogen Botrytis cinerea does not produce an elf18-like MAMP and its colonization in Arabidopsis is not affected by the lack of EFR (Brutus et al., 2010). Consistently, when $B$. cinerea was inoculated onto WT and transgenic K1A and K5A 
leaves, no differences in disease severity was observed (Fig. S6), indicating that the ectodomain of EFR-Cf-9 does not recognize any MAMP from $B$. cinerea and that basal defence is not enhanced by the presence of the chimera.

Taken together, these data indicate that pathogen-derived elf18-like MAMPs activate EFR-Cf-9-dependent immunity resulting in an HR, which restricts (hemi)biotrophic bacteria colonization, but does not affect colonization by necrotrophic bacteria or fungi.
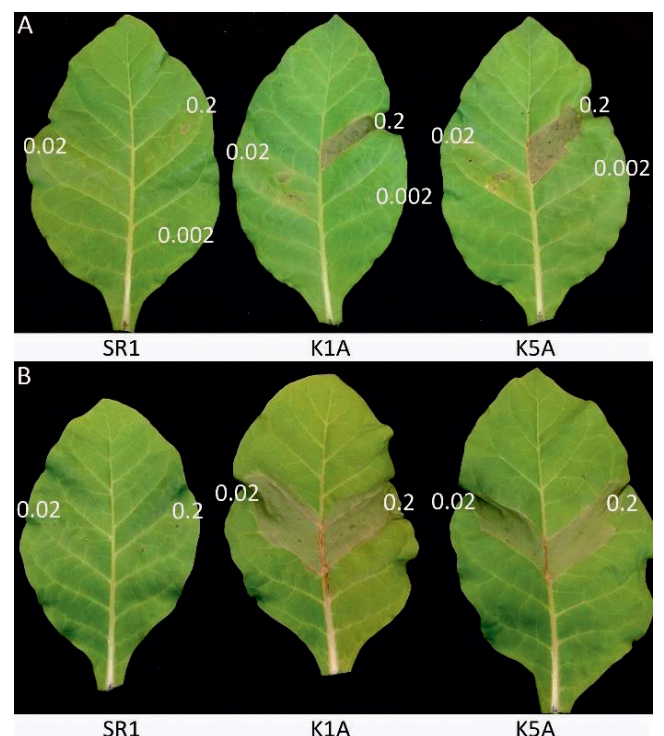

Figure 8. EFR-Cf-9 transgenic plants show hypersensitive response to high doses of bacterial pathogens. Leaf sectors of fully expanded leaves of four-week-old untransformed plants (WT) and EFR-Cf-9 K1A and K5A transgenic plants were inoculated with (A) Pta 11528 at $\mathrm{OD}_{600}$ of $0.002,0.02$ or 0.2 (about $10^{4}$, $10^{5}$ or $10^{6} \mathrm{CFU} / \mathrm{mL}$, respectively), or (B) with Pectobacterium carotovorum at $\mathrm{OD}_{600}$ of 0.02 or 0.2 , corresponding to a bacterial density of about $10^{5}$ or $10^{6} \mathrm{CFU} / \mathrm{mL}$, respectively ( $\mathrm{n}>3$ for each treatment). Pictures were taken at $48 \mathrm{~h}(\mathrm{~A})$ or $24 \mathrm{~h}$ (B) after inoculation. Experiments were repeated three times with similar results, and representative images are shown. Images of representative leaves are shown.

\section{Discussion}

\section{The chimera EFR-Cf-9 recognizes elf18 to trigger a strong immune response}

Interfamily transfer of PRRs is a promising strategy to confer broad spectrum resistance to pathogens, and it has been successfully employed with EFR, Xa21 and Ve1 (Afroz et al., 2011; Holton et al., 2015; Lacombe et al., 2010; Mendes et al., 2010; Schwessinger et al., 2015; Song et al., 2017b; Tripathi et al., 2014). Engineering chimeric receptors that combine the properties of two separate PRRs is another effective strategy to improve host resistance. Current research on chimeric PRRs has revealed that the ectodomain determines ligand specificity, while the endodomain determines output intensity (Brutus et al., 2010; He et al., 2000; Holton et al., 2015; Kishimoto et al., 2010; Kouzai et al., 2013; Schwessinger et al., 2015). Here we generated the chimera EFR-Cf-9 (Fig. 1), which combines the ectodomain of EFR that 
provides broad-spectrum recognition of bacterial pathogens, with the endodomain of Cf-9 that induces a strong HR-associated immune response. We demonstrated that, when expressed in tobacco plants, EFR-Cf-9 recognizes elf18 to trigger an HR (Fig. 2). We also showed that, similar to the Cf proteins (Liebrand et al., 2013; Postma et al., 2016), EFR-Cf-9 interacts with SOBIR1 (Fig. 3) and requires both SOBIR1 and BAK1 for its function (Fig. 4). In addition, EFR-Cf-9 also recognizes pathogen-derived elf18 or elf18-like MAMPs to activate immunity (Fig. 7) and triggers an HR during bacterial infection (Fig. 8). Moreover, EFR-Cf-9 retains the recognition specificity of EFR, as this chimera triggers a stronger response upon treatment with elf18C in comparison to elf18 variants with lower EFR-eliciting activity (Fig. S4 and S5).

In contrast to EFR-Cf-9, FLS2-Cf-9 failed to trigger an HR upon treatment with flg22 (Fig. S2), although FLS2-Cf-9 also interacts with SOBIR1 in N. benthamiana (Fig. 3). Since the FLS2 ectodomain is functional in FLS2-EFR (Brutus et al., 2010) and the Cf9 endodomain is functional in EFR-Cf-9 (Fig. 2), it is possible that the selected point of junction between the ectodomain of FLS2 and the endodomain of Cf-9 might not be optimal for functionality of this specific chimera.

\section{Early transduction events mediated by the EFR-Cf-9 chimera are similar to those employed by Cf-9}

Although interfamily PRR transfer and expression of chimeric PRRs can provide broad resistance to pathogens (Afroz et al., 2011; Holton et al., 2015; Lacombe et al., 2010; Schoonbeek et al., 2015), the downstream signalling components employed by these receptors are barely known. Studies have revealed that the signalling partners downstream of the perception event are not always conserved in the recipient plant. For example, OsSERK2, which is phylogenetically closely related to AtSERK1 and AtSERK2, is required for the functionality of transgenically expressed EFR in resistance to $X$. oryzae pv. oryzae (Xoo) in rice (Chen et al., 2014). However, in Arabidopsis, AtSERK1 and AtSERK2 are not required for EFR function (Roux et al., 2011), indicating that EFR utilizes different SERKs in Arabidopsis and rice. In addition, specific downstream signalling components may act in an opposite manner in different species. For instance, the Xa21-binding (XB) protein OsXB24 is an ATPase that negatively affects both Xa21- and EFR-mediated immunity in rice (Chen et al., 2010; Schwessinger et al., 2015), whereas its orthologue plays a positive role in EFRmediated immunity in Arabidopsis (Holton et al., 2015).

Ectopic expression of chimeric receptors allows to address the requirement of specific protein domains for interaction with partners participating in downstream signalling. For example, both EFR-Xa21 and native Xa21 form a constitutive complex with OsSERK2, 
indicating that the association of OsSERK2 with Xa21 does not specifically require the Xa21 ectodomain. In Arabidopsis, both Xa21 and EFR-Xa21 interact with BAK1 in a ligand-dependent manner (Schwessinger et al., 2015). In this study, we show that EFRCf-9 constitutively interacts with SOBIR1 and requires SOBIR1 for its function (Fig. 3 and 4). EFR-Cf-9 is anticipated to also form a complex with BAK1 upon elf18 treatment, as the chimera also requires kinase-active BAK1 for its function (Fig. 5). It should be noted that AtBAK1-5 had a stronger effect than AtBAK1-RD/N on suppressing EFRCf-9-mediated HR (Fig. 5). The interaction between AtBAK1 and EFR is kinase activityindependent and EFR has a higher affinity to AtBAK1-5 than to wild-type AtBAK1 (Schwessinger et al., 2011), explaining why AtBAK1-5 is more efficient than AtBAK1$\mathrm{RD} / \mathrm{N}$ in suppressing EFR-Cf-9 activity.

SOBIR1 constitutively interacts with $\mathrm{Cf}-4$, which shares an identical endodomain with Cf-9 (Thomas et al., 1997), whereas it does not interact with the RLKs FLS2 and EFR and is not required for RLK-mediated immunity (Gust and Felix, 2014; Liebrand et al., 2013; Liebrand et al., 2014). The external juxtamembrane region of EFR-Cf-9 does not carry the typical stretch of acidic amino acids that are thought to play a role in the interaction of Cf-9 with SOBIR1 (Bi et al., 2016; Gust and Felix, 2014). However, EFRCf-9 does interact with SOBIR1, suggesting that the TM of Cf-9, which contains an

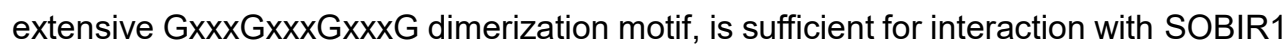
(Bi et al., 2016). The requirement of the GxxxG motif for the interaction between SOBIR1 and EFR-Cf-9 needs to be investigated by performing site-directed mutagenesis of this motif in future studies. Together, this indicates that the endodomain, and in particular the TM, of Cf- 9 provides the chimera EFR-Cf- 9 with the features necessary for Cf-9/Cf-4 signalling. These features enable EFR-Cf-9 to constitutively interact with SOBIR1, form a ligand-dependent complex with BAK1, and eventually mount an HR.

\section{Transgenic expression of EFR-Cf-9 affects resistance against bacterial pathogens}

In tomato, Cf proteins mediate a strong resistance to the $C$. fulvum strains carrying the proper avirulence genes (Joosten et al., 1997; Stergiopoulos and de Wit, 2009). It is widely accepted that this resistance is largely based on the HR resulting from the activation of the $\mathrm{Cf}$ protein, which leads to localized cell death that restricts pathogen spread. However, the strong resistance mediated by $\mathrm{Cf}$ genes has a narrow specificity and is easily overcome by mutations in the corresponding Avr genes of the pathogen (van den Burg et al., 2003). On the other hand, PRR-mediated immunity triggered upon recognition of MAMPs is effective against a wide range of microbes but is weaker than that of Cf proteins (Boutrot and Zipfel, 2017). Hence, the generation of a chimera 
containing the ectodomain of a PRR and the endodomain of a Cf protein might combine the beneficial features of PRRs and Cf proteins and enable the chimera to recognize MAMPs and mount an HR, leading to a strong immunity to a wide range of microbes.

EFR-Cf-9 recognizes elf18 to mount an HR (Fig. 2), suggesting that it might also recognize pathogen-derived elf18 to activate immunity. Indeed, transgenic tobacco plants expressing EFR-Cf-9 showed a significantly reduced susceptibility to two (hemi)biotrophic bacterial pathogens, Pta 11528 and Pst DC3000 (Fig. 7A and B). In addition, inoculation of a high dose of Pta 11528 causes an HR in the transgenic plants (Fig. 8A), indicating that EFR-Cf-9 recognizes both the purified elf18 peptide and the pathogen-derived EF-Tu, and that this recognition effectively restricts bacterial growth. However, compared to Pst DC3000, EFR-Cf-9-mediated resistance to Pta 11528 is less efficient (Fig. 7A and B), suggesting that Pta 11528 might partially suppress the EFR-Cf-9-mediated immune response.

Inoculation of the EFR-Cf-9-transgenic plants with the necrotrophic bacterium $P$. carotovorum also resulted in HR-like symptoms (Fig. 8B), since P. carotovorum carries a form of EF-Tu that is able to activate EFR (Lacombe et al., 2010). The EFR-Cf-9triggered HR was expected to promote the growth of this necrotrophic bacterium. However, no significant difference in $P$. carotovorum colonization between WT and transgenic plants was found at $24 \mathrm{~h}$ post-inoculation (Fig. 7C). Possibly, the EFR-Cf-9triggered HR promotes colonization by $P$. carotovorum at a later time point.

\section{Conclusion}

This work indicates that the EFR-Cf-9 chimera is functional in tobacco plants, since it recognizes elf18 to trigger an $\mathrm{HR}$, which fits our current view on the ectodomain of a PRR determining the ligand specificity, and the endodomain determining the output intensity. In addition, EFR-Cf-9 constitutively interacts with SOBIR1, and the EFR-Cf9-mediated HR triggered by elf18 is dependent on SOBIR1 and BAK1. These observations are reminiscent of our working model for Cf-4/Cf-9 and indicate that the endodomain of Cf-9 confers EFR-Cf-9 the repertoire of Cf-9/Cf-4-like signalling. Moreover, transgenic tobacco plants expressing EFR-Cf-9 do not show altered basal defence but are resistant to (hemi)biotrophic bacteria, suggesting a potential biotechnological use of this chimeric receptor to improve crop disease resistance. Furthermore, our construction of the EFR-Cf-9 chimera reveals that it is possible to customize crop immunity by the generation of chimeric receptors containing features of a varying ligand recognition range and immune response intensity. 


\section{Materials and Methods}

\section{Plant materials and growth conditions}

Nicotiana benthamiana, N. tabacum Samsun, N. tabacum SR1, Avr9-transgenic N. tabacum SR1 (Hammond-Kosack et al., 1998) and EFR-Cf-9-transgenic N. tabacum SR1 plants were grown under $16 \mathrm{~h}$ of light at $25^{\circ} \mathrm{C}$, and $8 \mathrm{~h}$ of darkness at $21^{\circ} \mathrm{C}$ in climate chambers with a relative humidity of $75 \%$.

\section{Constructs}

The DNA fragments representing the coding sequence (CDS) of the ectodomain of EFR (Lys649 of the transmembrane and cytoplasmic domain of Cf-9 by splicing overlapping extension polymerase chain reaction (SOE-PCR) (Higuchi et al., 1988), to generate DNA fragments encoding EFR-Cf-9 and FLS2-Cf-9, respectively, and cloned into the binary vector $\mathrm{pBI} 121$ under the control of the $35 \mathrm{~S}$ promoter. In addition, these two fragments were also inserted into pK7FWG2.0 to generate 35S:EFR-Cf-9-eGFP and 35S:FLS2-Cf-9-eGFP, and inserted into pGWB20 to generate 35S:EFR-Cf-9-10xMyc and 35S:FLS2-Cf-9-10xMyc.

Construction of SISOBIR1-eGFP, AtSOBIR1-RD/N-eGFP, SISOBIR1-Myc, Cf-4-Myc, FLS2-Myc and EFR-Myc was reported earlier (Liebrand et al., 2013). AtBAK1-5 and AtBAK1-RD/N were described in a published report (Schwessinger et al., 2011). Binary vectors carrying 35S:Cf-9 and 35S:Avr9 in pMOG800 have been described previously (van der Hoorn et al., 2000). All plasmids were transferred to Agrobacterium tumefaciens strain $\mathrm{C} 58 \mathrm{C} 1$, carrying the helper plasmid $\mathrm{pCH} 32$.

\section{Generation of transgenic tobacco plants}

Transformation of tobacco plants was performed according to established methods (Horsch et al., 1988). Briefly, A. tumefaciens carrying the 35S:EFR-Cf-9 plasmid were suspended in an infection medium composed as follows: Murashige-Skoog (MS) basal medium, 3\% w/v sucrose, $200 \mu \mathrm{M}$ acetosyringone, $0.001 \% \mathrm{v} / \mathrm{v}$ Silwet. Leaf discs of 1 $\mathrm{cm}$ in diameter from four-week-old tobacco SR1 leaves were incubated in the infection medium for $30 \mathrm{~min}$ at $25^{\circ} \mathrm{C}$. Leaf discs were then placed on co-culturing MS medium containing Gamborg's vitamin mix, $1 \mathrm{mg} / \mathrm{L}$ 6-BAP, $0.1 \mathrm{mg} / \mathrm{L}$ NAA, $200 \mu \mathrm{M}$ acetosyringone at $25^{\circ} \mathrm{C}$ in the dark for two days. Infected explants were then transferred to fresh solid MS medium supplemented with vitamin mix, $1 \mathrm{mg} / \mathrm{L}$ 6-BAP, $0.1 \mathrm{mg} / \mathrm{L} \mathrm{NAA}, 200 \mathrm{mg} / \mathrm{L}$ timentin, $200 \mathrm{mg} / \mathrm{L}$ cefotaxime and $200 \mu \mathrm{g} / \mathrm{mL}$ kanamycin at $25^{\circ} \mathrm{C}$ in the light for 30 days for shoot regeneration. Regenerated shoots were transferred to fresh solid MS medium supplemented with vitamin mix, $0.1 \mathrm{mg} / \mathrm{L}$ NAA, 
$200 \mathrm{mg} / \mathrm{L}$ timentin, $200 \mathrm{mg} / \mathrm{L}$ cefotaxime and $200 \mu \mathrm{g} / \mathrm{L}$ kanamycin at $25^{\circ} \mathrm{C}$ in the light for 30 days for root regeneration. From about 1200 co-cultivated leaf explants, 14 primary transformants were obtained and transferred to soil for propagation. Five transformants had detectable transgene expression and were propagated; of these, two (K1A and K5A) showed a 3:1 segregation ratio for kanamycin resistance and were therefore selected for further characterization.

\section{Hypersensitive response assays}

The elf18 (elf18D) and flg22 peptides were synthesized by EZBiolab (Carmel, IN, USA). The other elf18 peptides were kind gifts of Cyril Zipfel (The Sainsbury Laboratory, Cambridge, UK). For HR assays in N. tabacum SR1 and N. tabacum Samsun plants, fully expanded leaves were infiltrated with Agrobacterium suspensions at an $\mathrm{OD}_{600}$ of 1, except for Rx (D460V) that was infiltrated with an $\mathrm{OD}_{600}$ of 0.1 (Liebrand et al., 2013; Postma et al., 2016). Milli-Q water (MQ) and elf18 and/or flg22 peptides were infiltrated at 2 days post-infiltration (dpi) at the indicated concentrations. Leaves were examined for development of an HR between 2 and 12 dpi. For each treatment, at least three leaves taken from separate plants were agro-infiltrated.

\section{Virus-induced gene silencing (VIGS)}

VIGS plasmids pTRV1-RNA1, pTRV2-PDS, pTRV2-GUS, pTRV2-SISOBIR1/SOBIR1like and pTRV2-S/SERK3a/b were described previously (Liebrand et al., 2013; Postma et al., 2016). In brief, two-week-old N. tabacum Samsun plants were subjected to VIGS, for which Agrobacterium cultures harbouring a pTRV2 plasmid were mixed with pTRV1-RNA1 at a final OD600 of 0.8 . After about three weeks, fully expanded leaves were used for HR assays.

\section{Co-immunoprecipitation and immunoblot assays}

Co-IPs were performed as described previously (Liebrand et al., 2012). a-GFP-HRP (Miltenyi Biotec $\mathrm{GmbH}$ ) was used to detect eGFP-tagged proteins, while a-cMYC (Santa Cruz Biotechnology) and a-Mouse-HRP (GE Healthcare) were used to detect Myc-tagged proteins.

\section{Pathogenicity assays}

Pseudomonas amygdali pv. tabaci (Pta) 11528 and P. syringae pv. tomato (Pst) DC3000 were grown in $5 \mathrm{~mL}$ of low salt Bertani-Luria (LSBL) medium at $28^{\circ} \mathrm{C}$ for $24 \mathrm{~h}$ at $200 \mathrm{rpm}$ until $\mathrm{OD}_{600}$ was 1 . Bacteria were centrifuged for $10 \mathrm{~min}$ at 2,500xg, the supernatant was discarded, and bacteria were rinsed twice with sterile water. Bacteria were suspended in an infiltration medium containing $10 \mathrm{mM} \mathrm{MgCl}_{2}$ at the indicated doses. 
Pectobacterium carotovorum subsp. carotovorum strain DSMZ 30169 was obtained from DSMZ GmbH (Germany). Bacteria were grown in liquid LB at $28^{\circ} \mathrm{C}$ for $16 \mathrm{~h}$, centrifuged at $8,000 \times g$ for $10 \mathrm{~min}$, and washed with sterile water. Bacteria were then suspended in $50 \mathrm{mM}$ potassium phosphate buffer, $\mathrm{pH} 7.0$ and inoculated at the indicated doses.

Bacteria were syringe-infiltrated into leaf sectors of four-week-old tobacco plants (six sectors per leaf, three leaves per genotype). Inoculated plants were returned to greenhouse and bacterial count was determined after 0 and 120h (for Pta 11528 and Pst DC3000) or $24 \mathrm{~h}$ (for $P$. carotovorum). Briefly, inoculated leaf sectors were sterilized for $1 \mathrm{~min}$ in $\mathrm{EtOH} \mathrm{70 \% ,} \mathrm{washed} \mathrm{for} 1 \mathrm{~min}$ in sterile water, and leaf discs (diameter = $0.4 \mathrm{~mm}$ ) were cut from each sector. Discs were ground with a pestle in $100 \mu \mathrm{L}$ of sterile water, then additional $900 \mu \mathrm{L}$ of water were added; samples were vortexed, and serial dilutions were plated on LB solid medium. Plates were incubated at $28^{\circ} \mathrm{C}$ for two days, and colonies were counted for each dilution.

Detached tobacco leaves were inoculated with Botrytis cinerea strain SF1, which was isolated from cabbage (Ferrari et al., 2003a), as previously described (Manfredini et al., 2005).

\section{Expression analysis of the EFR-Cf-9 chimera and the defence-related genes HIN1 and ACRE132}

For analysis of the expression of EFR-Cf-9, total RNA was extracted from leaf sectors of four-week-old plants with Tri-reagent (Sigma) and treated with Turbo-DNase I (Ambion). First-strand cDNA was synthesized using ImProm-II Reverse Transcriptase (Promega, Madison, WI, USA). Expression of EFR-Cf-9 was evaluated by PCR using specific primers (5'-CAAATTCCATCCCTCGCTTA-3' and 5'TCTTTTCTTGTGCTTTTTCATTTTC-3'). The tobacco actin gene Tob66 (accession $\mathrm{n}$. U60491) was amplified using the following primers: 5'-CTGCCATGTATGTTGCTATT-3' and 5'-AGTCTCCAACTCTTGCTCAT-3'.

For quantitative analysis of HIN1 and ACRE132 expression, leaf sectors from fourweek-old plants were infiltrated with water or elicitors at the concentration of $100 \mathrm{nM}$. After 48h, infiltrated leaf sectors were collected from 3 separate plants for each genotype. Total RNA was extracted using NucleoZol reagent (Machery-Nagel GmbH, Düren, Germany), treated with RQ1 DNase (Promega), and first-strand cDNA was synthesized using ImProm-II reverse transcriptase (Promega). qRT-PCR analysis was performed using a CFX96 Real-Time System (Bio-Rad), using the GoTaq Real-Time PCR System (Promega). Three technical replicates were performed for each sample, 
and data were analysed with LinRegPCR, developed at Amsterdam University Medical Centers (AMC) (Ruijter et al., 2009). Expression levels of each gene, relative to EF1a, were determined as previously described (Ferrari et al., 2006), and expressed in arbitrary units. Primer pairs were the following: HIN1, 5'CTGCAACCCATGTAGCTGTC-3' and 5'-TGTGGTGGACAAATCGAACT-3'; ACRE132, 5'-GCTGGCGGTTATCAAGAAGT-3' and 5'-TGAAACCCATGATTGCATTT3'. EF1a, 5'-GCTCCCACTTCAGGATGTTT-3' and 5'-CCAACATTGTCACCAGGAAG3'.

\section{Mass spectrometry}

Membrane-enriched protein fractions from one leaf of a WT SR1 plant and of a transgenic K1A plant were extracted as previously described (Mattei et al., 2016). Proteins were separated by SDS-PAGE, and lanes were cut into 10 slices, that were subjected to in-gel trypsin digestion. Peptides were analysed by LTQ liquid chromatography (LC) Orbitrap MS/MS, and protein identification was performed by MaxQuant platform and Proteome Discoverer as previously described (Mattei et al., 2016).

\section{Acknowledgements}

We would like to thank Cyril Zipfel (The Sainsbury Laboratory, Cambridge, UK) for providing elf18 peptides, Pierre J.G.M. de Wit (Wageningen University \& Research, The Netherlands) for the plasmids carrying 35S:Cf-9 and 35S:Avr9, and Jonathan Jones (The Sainsbury Laboratory, Cambridge, UK) for providing the tobacco line expressing Avr9. This work was supported by the Institute Pasteur-Fondazione Cenci Bolognetti, by the Ministero dell'Istruzione, dell'Università e della Ricerca, by ERA-Net (ERA-CAPS project "SIPIS") and by Sapienza Università di Roma (Progetti di Ricerca 2016 and 2017). J. Wu is supported by a scholarship from the China Scholarship Council (CSC). I-B. Reca was supported by the SIMISA project from the Ministero dello Sviluppo Economico as CNR-ISPA research fellow. The authors have no conflict of interest to declare. 


\section{Supplementary information}

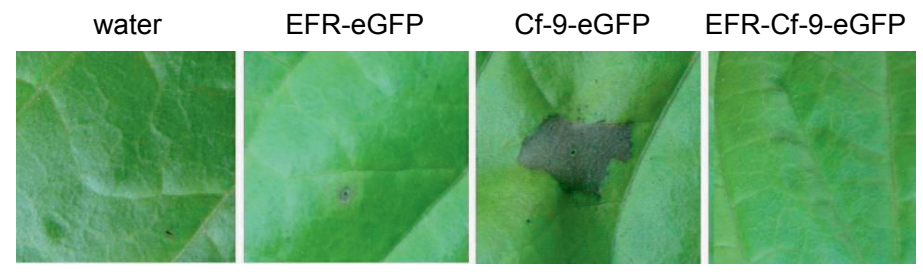

Avr9 transgenic N. tabacum

Figure S1. EFR-Cf-9 does not trigger an HR in tobacco plants expressing Avr9. eGFP-tagged EFR, Cf-9 and EFR-Cf-9 were transiently expressed in Avr9-transgenic N. tabacum SR1 plants at a final $\mathrm{OD}_{600}$ of 1. For each construct, at least three leaves taken from separate plants were agro-infiltrated. Pictures were taken at 3 days post-infiltration (dpi). The infiltrated areas are indicated by white dashed lines. Under these conditions, all leaves agro-infiltrated with Cf-9-eGFP, and none of the other samples, showed necrosis of at least half of the infiltrated area. This experiment was repeated three times with similar results. Representative images are shown.

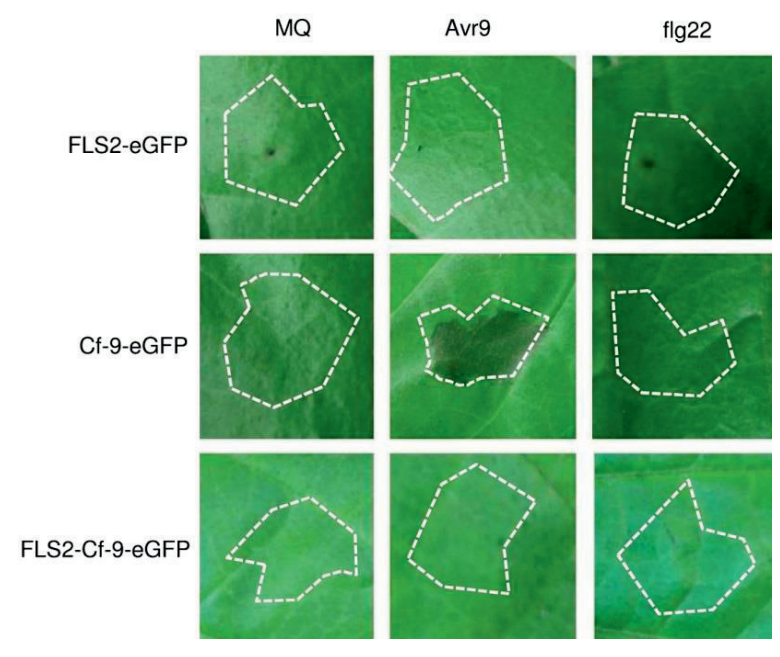

Figure S2. FLS2-Cf-9 does not trigger an HR in tobacco plants upon treatment with flg22. eGFPtagged FLS2, Cf-9 and FLS2-Cf-9 were either expressed alone (first and third column) or transiently coexpressed with Avr9 (second column) and followed by treatment with Milli-Q (MQ) water (first column) or $100 \mu \mathrm{M}$ flg22 (third column) after two days. Agrobacteria driving expression of the various constructs were infiltrated at a final OD600 of 1 . Pictures were taken at 4 dpi with the elicitor or at seven days of co-expression with Avr9. The infiltrated areas are indicated by white dashed lines. For each treatment, at least three leaves per construct, taken from separate plants, were infiltrated. Under these conditions, all leaves co-expressing Avr9 and Cf-9-eGFP showed necrosis of at least half of the infiltrated area, whereas none of the other samples showed HR-like symptoms. This experiment was repeated three times with similar results. Representative images are shown. 
EFR SP

Cf-9 TM/iJM

MKLSFSLVFNALTLLLQVCIFAQA

RFSNETDMQALLEFKSQVSENNKREVLASWNHSSPFCNWIGVTCGR RRERVISLNLGGFKLTGVISPSIGNLSFLRLLNLADNSFGSTIPQKVGR LFRLQYLNMSYNLLEGRIPSSLSNCSRLSTVDLSSNHLGHGVPSELG SLSKLAILDLSKNNLTGNFPASLGNLTSLQKLDFAYNQMRGEIPDEVAR LTQMVFFQIALNSFSGGFPPALYNISSLESLSLADNSFSGNLRADFGYL LPNLRRLLLGTNQFTGAIPKTLANISSLERFDISSNYLSGSIPLSFGKLR NLWWLGIRNNSLGNNSSSGLEFIGAVANCTQLEYLDVGYNRLGGELP ASIANLSTTLTSLFLGQNLISGTIPHDIGNLVSLQELSLETNMLSGELPV

EFR LRR SFGKLLNLQVVDLYSNAISGEIPSYFGNMTRLQKLHLNSNSFHGRIPQ SLGRCRYLLDLWMDTNRLNGTIPQEILQIPSLAYIDLSNNFLTGHFPEE VGKLELLVGLGASYNKLSGKMPQAIGGCLSMEFLFMQGNSFDGAIPD ISRLVSLKNVDFSNNNLSGRIPRYLASLPSLRNLNLSMNKFEGRVPTT GVF

EFR eJM RNATAVSVFGNTNICGGVREMQLKPCIVQASPRKRKPLSVRKK

WQGVLVGYGCGLVIGLSVIYIMWSTQYPAWFSRMDLKLEHIITTKMKK HKKRY

Figure S3. Identification of EFR-Cf-9 in microsomal fractions of transgenic tobacco plants. Amino acid sequence of the EFR-Cf- 9 chimeric receptor. The five peptides identified by mass spectrometry in microsomal protein fractions extracted from transgenic EFR-Cf-9-expressing K1A leaves are underlined. $\mathrm{SP}$, signal peptide; LRR, leucine-rich repeat; eJM, external juxtamembrane; TM, transmembrane; iJM, internal juxtamembrane.

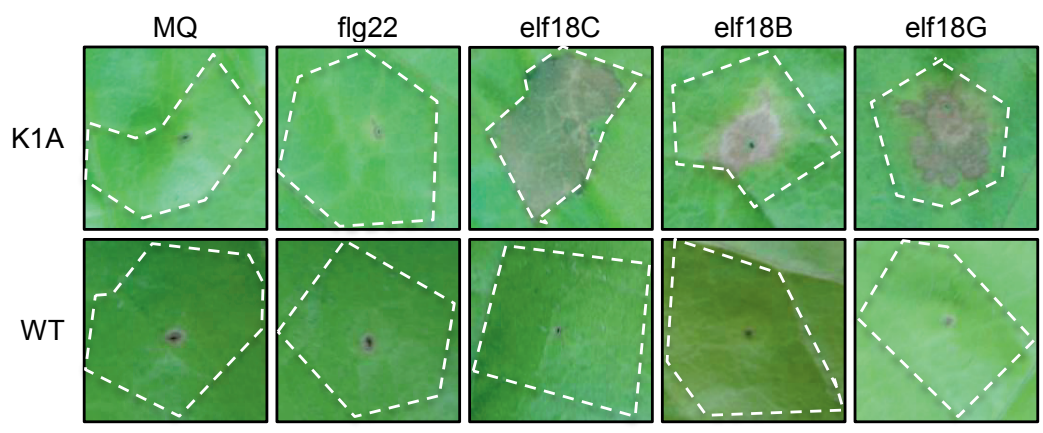

Figure S4. EFR-Cf-9-transgenic plants recognize different elf18 variants. Fully expanded leaves of four-week-old untransformed (WT) and transgenic tobacco plants expressing EFR-Cf-9 (K1A) plants were treated with Milli-Q (MQ) water or with flg22, elf18C, elf18B or elf18G at a concentration of 100 $\mathrm{nM}$. For each peptide, at least four leaves per genotype taken from independent plants were infiltrated. Pictures were taken at 40 hours post-infiltration. The infiltrated area is indicated by the white dashed line. At this time point, all leaves from K1A plants infiltrated with el18C, elf18B or elf18C showed necrosis of at least half of the infiltrated area, whereas those infiltrated with flg22 or water, and all leaves from WT plants, failed to display any HR-like symptoms. This experiment was repeated three times with similar results, and representative images are shown. 


\section{Chapter 4}
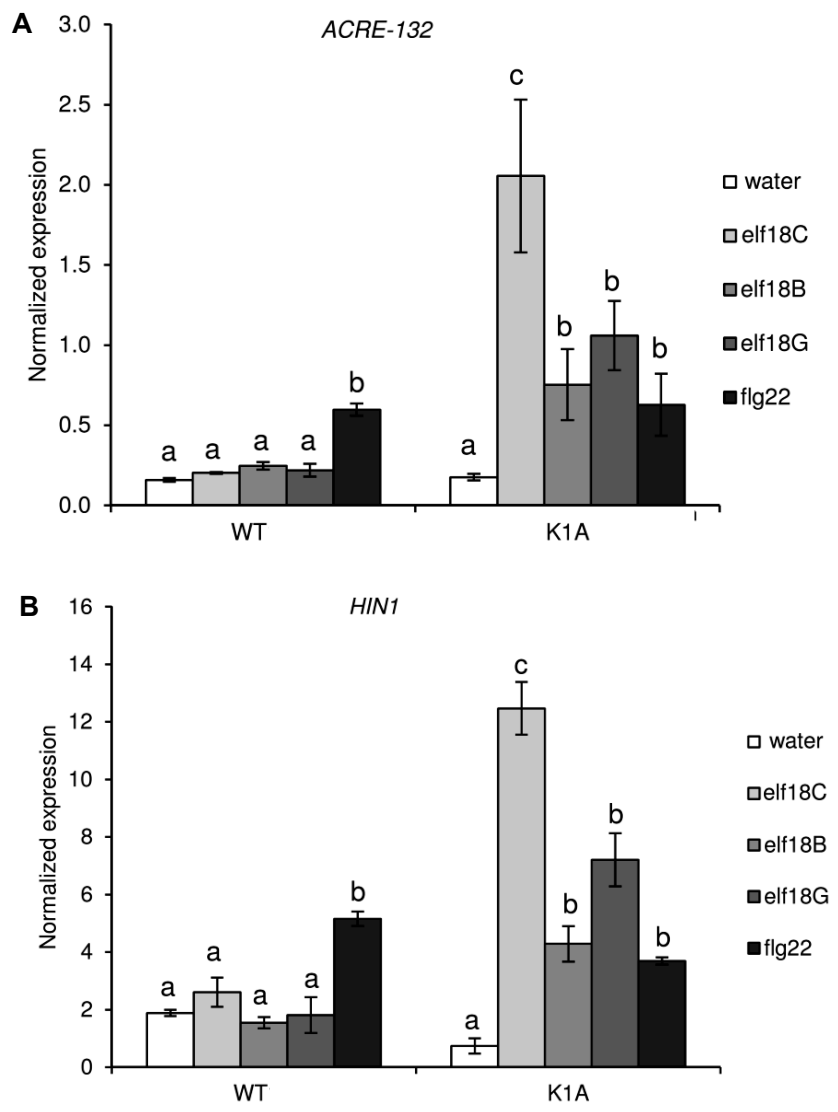

Figure S5. Defence-related genes $A C R E 132$ and $H I N 1$ are differentially up-regulated in transgenic plants expressing EFR-Cf-9 upon treatment with elf18 variants. Quantitative RT-PCR was used to determine ACRE-132 (A) and HIN1 (B) expression levels in WT and EFR-Cf-9-expressing transgenic $\mathrm{K} 1 \mathrm{~A}$ plants upon treatment with Milli-Q (MQ) water or with elf18C, elf18B, elf18G and flg22 at a concentration of $100 \mathrm{nM}$. RNA was extracted from infiltrated leaf sectors obtained from three separate plants for each genotype. Bars represent average $\pm S D$ of three replicates. Different letters indicate statistically significant differences, according to one-way ANOVA followed by Tukey's significance test $(P<0.05)$. This experiment was repeated twice with similar results.

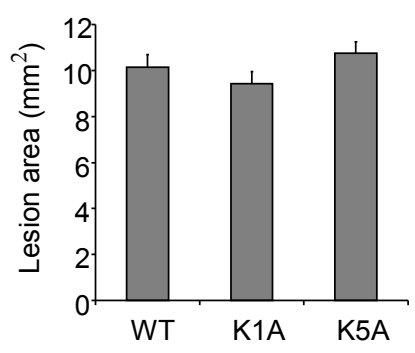

Figure S6. Transgenic plants expressing EFR-Cf-9 show unaltered susceptibility to Botrytis cinerea. Leaves of soilgrown four-week-old WT and transgenic plants expressing EFRCf-9 (K1A and K5A) were inoculated with $B$. cinerea spore suspension, and lesion areas were measured at 48 hours postinfiltration. Bars indicate average lesion area \pm standard error $(n>$ 18). No significant difference was observed between $W T$ and transgenic lines (K1A and K5A), according to Student's $t$-test $(P>$ $0.5)$. 


\section{Chapter 5}

Functional analysis of CLV2-containing complexes and identification of components required for the response to the CLV3 peptide in Arabidopsis thaliana

Jinbin $\mathrm{Wu}^{1}$, Xiaoyu Hou${ }^{1}$, Jiajing Wang ${ }^{2}$, Guodong Wang ${ }^{2}$ and Matthieu H.A.J. Joosten ${ }^{1}$

${ }^{1}$ Laboratory of Phytopathology, Wageningen University \& Research, Droevendaalsesteeg 1, 6708 PB Wageningen, The Netherlands

${ }^{2}$ College of Life Sciences, Shaanxi Normal University, 710119 Xi'an, China 


\section{Abstract}

Receptor-like proteins (RLPs) and receptor-like kinases (RLKs) are cell surface receptors that regulate immunity and development in various plant species. RLPs are structurally similar to RLKs, but lack an intracellular kinase domain to activate downstream signalling components. In plant immunity, both RLPs and RLKs form ligand-induced complexes with the regulatory RLK BRI1-ASSOCIATED RECEPTOR KINASE 1 (BAK1) to activate downstream signalling. In addition to interacting with BAK1, RLPs also constitutively interact with the regulatory RLK SUPPRESSOR OF BIR1-1 (SOBIR1) and require this RLK to activate downstream immune signalling. The RLP CLAVATA2 (CLV2) is involved in regulation of plant development, which is mediated by the secreted endogenous CLV3 peptide in various plant species. Previously, an interaction between the tomato orthologues of CLV2 and SOBIR1 was reported, but the significance of this interaction remains unknown. As the function of SOBIR1 and CLV2 is conserved across plant species, we aimed to investigate the significance of this interaction in Arabidopsis thaliana (further referred to as Arabidopsis). In this study, we observed that Arabidopsis CLV2 interacts with SOBIR1, as well as with BAK1. Our studies on both SOBIR1/BAK1-dependent immune responses and CLV2-dependent CLV3 signalling revealed that CLV2 affects the immune response, whereas SOBIR1 participates in the CLV2-dependent regulation of the rosette leaf number. Additionally, we aimed to identify downstream components required for CLV3 signalling. Since the Arabidopsis G-protein $\beta$ subunit AGB1 and the G-protein $y$ subunits $A G G 1$ and $A G G 2$ are required for CLV3 signalling in the shoot and for signalling triggered by the well-characterized RLK FLAGELLIN-SENSING 2 (FLS2), which mediates defence against bacterial pathogens, we speculated that other regulators of FLS2 signalling might also play a role in CLV3 signalling. With the usage of the root growth inhibition assay employing a synthetic peptide of CLV3 (CLV3p), which is a routine assay to study the involvement of components in the CLV3-triggered signalling pathway, we observed that these G-protein subunits are also required for the response to CLV3p in the root. However, Ga-containing subunits, which associate with the $G \beta$ and $G y$ subunits to form a typical heterotrimeric complex, are not required for CLV3 signalling in the root. We furthermore found that other tested regulators of FLS2 signalling are not involved in the signalling pathway triggered by CLV3p in the root. 


\section{Introduction}

Receptor-like proteins (RLPs) and receptor-like kinases (RLKs) containing leucine-rich repeats in their ectodomains are the two major classes of cell surface plant receptors, and recognize extracellular signals to regulate immunity and development (Boutrot and Zipfel, 2017; Jamieson et al., 2018; van der Burgh and Joosten, 2019; Wang et al., 2010a). Compared with RLKs, RLPs lack an intracellular kinase domain to activate downstream components. When involved in immunity, RLPs and RLKs recognize extracellular immunogenic patterns, which include so-called microbe-associated molecular patterns (MAMPs) and pathogen-derived extracellular effectors, to initiate extracellularly-triggered immunity (Dodds and Rathjen, 2010; Jones and Dangl, 2006; van der Burgh and Joosten, 2019). Furthermore, RLKs also recognize secreted endogenous signals to regulate various developmental processes, yet specific ligands for RLPs involved in development have not been reported (Jamieson et al., 2018; Wang et al., 2010a).

RLP-mediated immunity has been demonstrated in various plant species (Boutrot and Zipfel, 2017; van der Burgh and Joosten, 2019; Wan et al., 2019). For instance, the tomato (Solanum lycopersicum, SI) RLP Cf-4 confers resistance to strains of the biotrophic fungus Cladosporium fulvum secreting the effector Avr4 (Joosten et al., 1994; Thomas et al., 1997). In Arabidopsis thaliana (At, further referred to as Arabidopsis), RLP23 mediates defence against infection by a broad range of pathogens producing the MAMP NECROSIS- AND ETHYLENE-INDUCING PROTEIN 1 (NEP1)-like proteins (NLPs), and recognition of the immunogenic peptide NLP24 triggers the production of reactive oxygen species (ROS) (Albert et al., 2015; Oome et al., 2014). Another example is the observation that Arabidopsis RLP42 recognizes the MAMP ENDOPOLYGALACTURONASE3 (PG3) of the necrotrophic fungus Botrytis cinerea to activate downstream immune responses, eventually leading to the development of necrotic symptoms (Zhang et al., 2014). It has been generally observed that RLPs, including Cf-4 and RLP23, constitutively interact with the regulatory RLK SUPPRESSOR OF BIR1-1 (SOBIR1), and form ligand-induced complexes with the regulatory RLKs, referred to as SOMATIC EMBRYOGENESIS RECEPTOR-LIKE KINASE (SERK) proteins, to activate downstream immune signalling (Albert et al., 2015; Domazakis et al., 2018; Du et al., 2015; Gust and Felix, 2014; Liebrand et al., 2013; Liebrand et al., 2014; Postma et al., 2016; van der Burgh and Joosten, 2019; Wang et al., 2018c; Zhang et al., 2013a). RLP42 also constitutively interacts with SOBIR 1 and requires this regulatory RLK to activate downstream immune signalling (Zhang et al., 2014). However, it was also shown that RLP42 signalling is not affected by a loss-of-function mutation in the gene encoding BRI1-ASSOCIATED RECEPTOR 
KINASE 1 (BAK1), which is also known as SERK3 (Zhang et al., 2014), probably due to functional redundancy of the various SERKs.

Besides playing a role in immunity, RLPs also function in development (Jamieson et al., 2018; Wang et al., 2010a). Arabidopsis CLAVATA2 (CLV2), also known as RLP10, is one of the most well-known RLPs involved in development and is involved in regulation of developmental processes mediated by various CLV3/ENDOSPERM SURROUNDING REGION (ESR)-related (CLE) members (Fiers et al., 2005; Jeong et al., 1999; Kayes and Clark, 1998; Miwa et al., 2008). CLV2 constitutively interacts with CORYNE/SUPPRESSOR OF OVEREXPRESSION OF LLP1-2 (CRN/SOL2, further referred to as $C R N$ ), which is a pseudokinase and lacks an obvious ectodomain, and both CLV2 and CRN require each other for their re-localization from the endoplasmic reticulum to the plasma membrane (Bleckmann et al., 2010; Miwa et al., 2008; Müller et al., 2008; Nimchuk et al., 2011; Zhu et al., 2010b). The CLV2/CRN complex is involved in perception of the well-studied CLE member CLV3, which a secreted peptide derived from stem cells in the shoot apical meristem (SAM) (Clark et al., 1995; Fletcher et al., 1999). Studies have revealed that CLV3 is primarily perceived by the RLK CLV1, which functions in parallel to the RLKs BARELY ANY MERISTEMs (BAMs) and RECEPTOR-LIKE PROTEIN KINASE 2/TOADSTOOL 2 (RPK2/TOAD2; further referred to as RPK2) (Casamitjana-Martinez et al., 2003; Clark et al., 1993; Clark et al., 1997; DeYoung et al., 2006; Kinoshita et al., 2010; Shinohara and Matsubayashi, 2015; Somssich et al., 2016b). However, the exact role of the CLV2/CRN complex in perception of CLV3 remains unclear (Somssich et al., 2016b), since CLV2 does not bind CLV3 (Shinohara and Matsubayashi, 2015) and the pseudokinase CRN lacks the ability to activate downstream signalling (Nimchuk et al., 2011).

In addition to playing a role in the shoot, the CLV2/CRN complex is also required for CLE signalling in the root (Fiers et al., 2005; Miwa et al., 2008). Both overexpression of various CLE genes and exogenous application of synthetic peptides corresponding to the conserved CLE domain of these CLEs inhibit root elongation (Fiers et al., 2005; Hobe et al., 2003; Kinoshita et al., 2007; Miwa et al., 2008). In addition, the CLV2/CRN complex is generally required for CLE-mediated suppression of root elongation, although the mechanism behind the role of CLV2/CRN is not clear (Fiers et al., 2005; Miwa et al., 2008; Müller et al., 2008). For instance, both overexpression of CLV3 and application of a synthetic CLV3 peptide (CLV3p) trigger a CLV2/CRN-dependent short root phenotype (Fiers et al., 2005; Miwa et al., 2008; Müller et al., 2008). In addition, CLV2 and CRN are also required for suppression of root elongation triggered by CLE9/10p (which corresponds to the identical CLE domain of CLE9 and CLE10, and is further referred to as CLE9p) and CLE45p, which inhibit the formation of protoxylem 
and protophloem, respectively (Depuydt et al., 2013; Kondo et al., 2011).

The RLK FLAGELLIN-SENSING 2 (FLS2) recognizes the MAMP flg22, which is a 22amino-acid immunogenic peptide of bacterial flagellin, to mediate defence against bacterial pathogens in various plant species (Felix et al., 1999; Gómez-Gómez and Boller, 2000; Hann and Rathjen, 2007; Robatzek et al., 2007; Takai et al., 2008; Zipfel et al., 2004). The FLS2 signalling pathway is well studied, and various positive and negative regulators of FLS2 signalling have been identified in Arabidopsis (Couto and Zipfel, 2016; Wan et al., 2019; Zhou et al., 2019). Concerning positive regulators of FLS2 signalling, for instance the regulatory RLK BAK1 functions as a co-receptor for FLS2 in recognizing flg22, which functions as a kind of molecular glue to stabilize the heterodimerization of the ectodomains of FLS2 and BAK1, resulting in the activation of the FLS2/BAK1 complex (Chinchilla et al., 2007; Schulze et al., 2010; Sun et al., 2013b). The receptor-like cytoplasmic kinase (RLCK) BOTRYTIS-INDUCED KINASE1 (BIK1) interacts with the kinase domains of both FLS2 and BAK1 (Lu et al., 2010; Zhang et al., 2010). Upon flg22 perception, activated BAK1 phosphorylates BIK1, which trans-phosphorylates, and subsequently dissociates from, the FLS2/BAK1 complex (Lin et al., 2014; Lu et al., 2010; Zhang et al., 2010). Activated BIK1 then phosphorylates the plasma membrane-associated NADPH oxidase RESPIRATORY BURST OXIDASE HOMOLOG D (RBOHD), which is the main enzyme mediating ROS production in FLS2 signalling (Kadota et al., 2014; Li et al., 2014b). The FLS2/BIK1 complex also associates with the Arabidopsis heterotrimeric G-protein complex consisting of the Ga-containing subunits EXTRA-LARGE G-PROTEIN 2 (XLG2) and XLG3, the G-protein $\beta$ subunit AGB1 and the G-protein $y$ subunits $A G G 1$ and AGG2 (further referred to as AGG1/2) (Liang et al., 2016). This G-protein complex promotes the stabilization of BIK1 in the resting state, and probably contributes to the activation of RBOHD upon flg22 perception (Liang et al., 2016; Wang et al., 2018a). Furthermore, perception of flg22 also activates downstream mitogen-activated protein kinase (MAPK) cascades, thereby initiating a series of downstream responses (Asai et al., 2002; Bi et al., 2018; Gao et al., 2008; Meng and Zhang, 2013). Concerning negative regulators of FLS2 signalling, for instance the pseudokinases BAK1-INTERACTING RECEPTOR-LIKE KINASE 2 (BIR2) and BIR3 both sequester BAK1 from FLS2 to negatively regulate the flg22-induced FLS2/BAK1 complex formation (Halter et al., 2014b; Imkampe et al., 2017). In addition, the serine/threonine protein phosphatase type 2C (PP2C) subunit PP2C38 dephosphorylates BIK1 and functions redundantly with its paralogue PP2C48 to negatively regulate FLS2 signalling (Couto et al., 2016).

Although various CLV3 receptors in the SAM have been identified, only a few downstream components required for CLV3 signalling are known (Somssich et al., 
2016b). For instance, the $G \beta$ subunit $A G B 1$ and the $G y$ subunits $A G G 1 / 2$ function downstream of CLV1, RPK2 and CLV2 to regulate CLV3 signalling in the shoot (Ishida et al., 2014). As AGB1 and AGG1/2 are also required for FLS2 signalling (Liang et al., 2016; Liang et al., 2018; Wang et al., 2018a), it implicates that other regulators of FLS2 signalling probably also play a role in CLV3 signalling. As mentioned above, various regulator of FLS2 signalling are known and a series of Arabidopsis mutants or transformants of these regulators have been reported (Couto and Zipfel, 2016; Wan et al., 2019; Zhou et al., 2019). In addition, the root length assay employing CLV3p offers a simple and direct method to screen for downstream components required for CLV3 signalling (Fiers et al., 2005). Therefore, examining the responsiveness of Arabidopsis mutants and transformants, which display impaired FLS2 signalling, to CLV3p should allow the identification of novel components required for CLV3 signalling in the root.

Previously, an interaction between the tomato orthologues of CLV2 and SOBIR1 was reported (Liebrand et al., 2013), however the significance of this interaction has remained unknown. As the roles of SOBIR1 and CLV2 are conserved across plant species (Albert et al., 2015; Kayes and Clark, 1998; Liebrand et al., 2013; Xu et al., 2015; Zhang et al., 2014), we aimed to investigate the significance of this interaction in Arabidopsis. In this study, an interaction between CLV2 and SOBIR1 from Arabidopsis is also observed. Since SOBIR1 is required for RLP-mediated immune signalling, while CLV2 is required for CLE signalling, we investigated whether CLV2 affects SOBIR1-dependent RLP-mediated immune signalling, and whether SOBIR1 plays a role in CLV2-dependent CLE signalling. Additionally, we also aimed to identify downstream components required for CLV3 signalling with the usage of the root length assay employing CLV3p, which allows efficiently screen for components required for CLV3 signalling (Fiers et al., 2005). Based on the speculation that some regulators of FLS2 signalling might also play a role in CLV3 signalling, we collected various Arabidopsis mutants or transformants displaying impaired FLS2 signalling and examined their responsiveness to exogenous application of CLV3p in the root.

\section{Results}

\section{CLV2 interacts with SOBIR1 when transiently co-expressed in Nicotiana benthamiana}

We first studied whether, next to the tomato orthologues, AtCLV2 also interacts with AtSOBIR1. Therefore, AtCLV2-eGFP was transiently co-expressed with AtSOBIR1Myc in leaves of $N$. benthamiana. Co-expression of $\beta$-glucuronidase (GUS)-eGFP and AtSOBIR1-Myc was included as a negative control, while co-expression of S/CLV2eGFP and SISOBIR1-Myc was included as a positive control. In addition, co- 
expression of the reciprocal combinations AtCLV2-eGFP and SISOBIR1-Myc, as well as SICLV2-eGFP and AtSOBIR1-Myc, was also included. All these different combinations were expressed in the presence of the silencing suppressor P19, which promotes overall protein accumulation (Voinnet et al., 2015). Immunoprecipitation of SICLV2-eGFP resulted in the co-purification of SISOBIR1-Myc (Fig. 1), which is consistent with the previous finding that SICLV2 interacts with SISOBIR1 (Liebrand et al., 2013). In addition, AtSOBIR1-Myc co-purified with AtCLV2-eGFP (Fig. 1), indicating that AtCLV2 also interacts with AtSOBIR1. Moreover, AtCLV2 and SISCLV2 also interact with SISOBIR1 and with AtSOBIR1, respectively (Fig. 1). Taken together, these observations indicate that the interaction between CLV2 and SOBIR1 is conserved in both Arabidopsis and tomato.

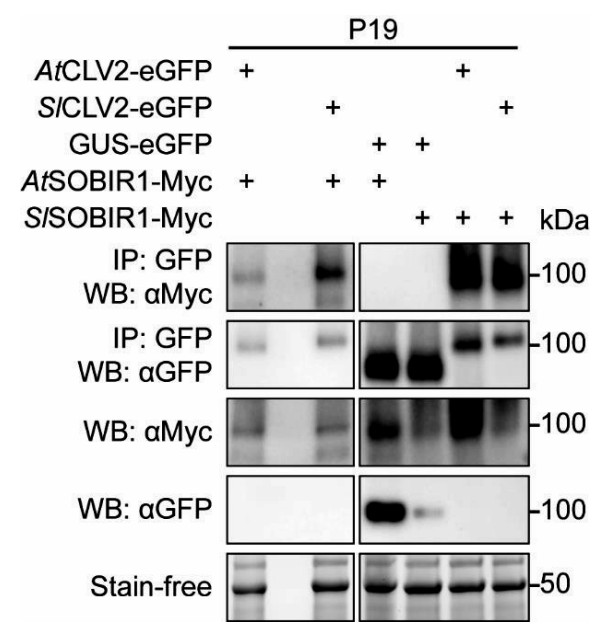

Figure 1. CLV2 interacts with SOBIR1 when transiently co-expressed in Nicotiana benthamiana. Both CLV2 and SOBIR1 from Arabidopsis thaliana (At) and tomato (Solanum lycopersicum, SI) were used. CLV2-eGFP or $\beta$-glucuronidase (GUS)-eGFP were co-expressed with SOBIR1-Myc in the presence of the silencing suppressor P19 in leaves of $N$. benthamiana. Proteins were extracted and subjected to immunoprecipitation (IP) by using GFP-affinity beads, followed by western blotting (WB). aGFP antibody was used to detect CLV2-eGFP and GUS-eGFP, and aMyc antibody was used to detect SOBIR1-Myc. Rubisco bands in the stain-free panel indicate equal loading. The experiment was repeated three times with similar results, and representative results are shown.

AtCLV2 and AtCRN participate in immune responses that are either dependent or independent of AtSOBIR1

As AtCLV2 (further referred to as CLV2) interacts with AtSOBIR1 (further referred to as SOBIR1) (Fig. 1), we studied whether CLV2 affects SOBIR1-dependent immune 
responses mediated by RLP42 and RLP23, and also studied whether SOBIR1 is involved in CLV2-dependent CLE signalling. AtCRN (further referred to as CRN) was also included in these studies, as CRN constitutively interacts with CLV2 and is required for CLV2-dependent CLE signalling (Bleckmann et al., 2010; Fiers et al., 2005; Miwa et al., 2008; Müller et al., 2008; Zhu et al., 2010b).

A
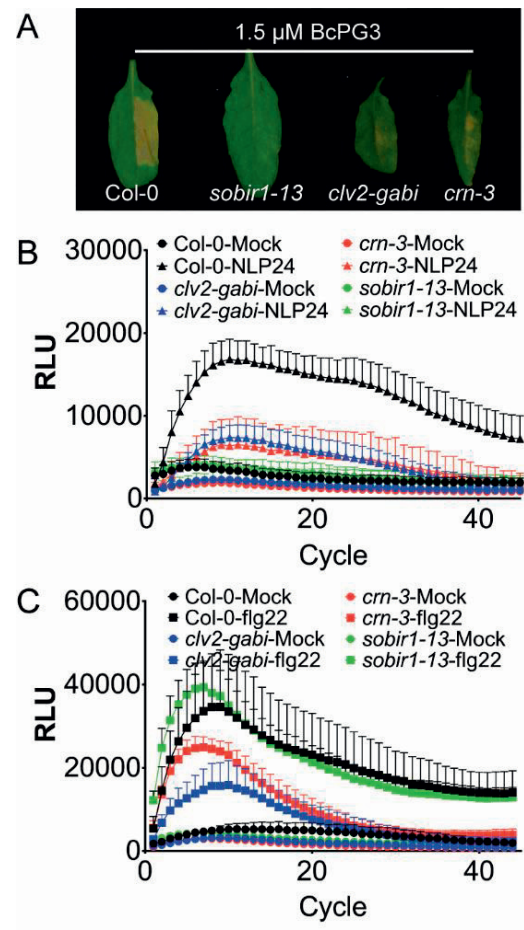

Figure 2. CLV2 and CRN participate in immune responses that are either dependent or independent of SOBIR1 in Arabidopsis. (A) CLV2 and CRN affect the development of BcPG3-triggered necrotic symptoms. Six rosette leaves from two plants of each line were infiltrated with a $1.5 \mu \mathrm{M} \mathrm{BcPG} 3$ protein solution, and pictures were taken at 6 days post-infiltration (dpi). (B, C) CLV2 and CRN affect the production of reactive oxygen species (ROS) triggered by NLP24 (B) and flg22 (C). Eight leaf discs from four leaves of two plants per line were used for the ROS assay. Milli-Q water was used as a control (Mock). These experiments were repeated three times with similar results, and representative results are shown.

Consistent with the published data that RLP42 recognizes BcPG3 to trigger SOBIR1dependent necrotic symptoms (Zhang et al., 2014), BcPG3 triggered necrotic symptoms in Arabidopsis accession Columbia (Col-0), whereas this necrosis was completely suppressed in the loss-of-function mutant sobir1-13 (further referred to as sobir1) (Fig. 2A). In addition, the development of BcPG3-triggered necrotic symptoms was also suppressed in the loss-of-function mutants clv2-gabi (further referred to as $c / v 2$ ) and crn-3 (further referred to as crn), but to a lesser extent than in the sobir1 mutant (Fig. 2A). We furthermore observed that the NLP24-triggered ROS burst was suppressed in the sobir1 mutant (Fig. 2B), which is consistent with the published data that RLP23 recognizes NLP24 to trigger a SOBIR1-dependent ROS burst (Albert et al., 2015). Moreover, the NLP24-triggered ROS burst was also suppressed in the clv2 and 
crn mutants, but again to a lesser extent than in the sobir1 mutant (Fig. 2B). The partially impaired SOBIR1-dependent RLP-mediated immune response in the clv2 and crn mutants might be caused by the functional redundancy of CLV2 and CRN orthologues (Wang et al., 2010b; Wu et al., 2016a). In addition, as CLV2 and CRN contribute to the accumulation level of their interactors (Hazak et al., 2017; Jeong et al., 1999), the SOBIR1 protein might accumulate at a lower level in the clv2 and crn mutants, resulting in an impaired RLP-mediated immune response. Taken together, these observations indicate that both CLV2 and CRN play a role in SOBIR1-dependent RLP-mediated immune responses in Arabidopsis.

To study whether the role of CLV2 and CRN in immunity is specific for SOBIR1dependent RLP signalling, we subsequently studied whether CLV2 and CRN affect the SOBIR1-independent FLS2 signalling. Consistent with the published data that SOBIR1 is not required for FLS2 signalling (Albert et al., 2015; Zhang et al., 2013a), the flg22triggered ROS burst was not affected in the sobir1 mutant (Fig. 2C). However, when compared with both Col-0 and the sobir1 mutant, the clv2 and crn mutants produced lower amounts of ROS upon treatment with flg22 (Fig. 2C), indicating that CLV2 and CRN do also play a role in the SOBIR1-independent FLS2 signalling. Possibly, CLV2 and CRN interact with co-receptors of FLS2, such as BAK1 (see below), thereby modulating FLS2 signalling.

\section{SOBIR1 participates in the CLV2/CRN-dependent regulation of the rosette leaf number}

The interaction between CLV2 and SOBIR1 indicates that SOBIR1 might play a role in CLV2/CRN-dependent developmental signalling. CLV2 and CRN are required for CLV3 signalling in the shoot, which negatively regulates the number of Arabidopsis rosette leaves and carpels, and the SAM size (Clark et al., 1995; Miwa et al., 2008; Müller et al., 2008). In addition, CLV2 and CRN are also required for suppression of root elongation triggered by various CLE peptides, including CLV3p, CLE9p and CLE45p (Depuydt et al., 2013; Fiers et al., 2005; Kondo et al., 2011; Miwa et al., 2008; Müller et al., 2008). Therefore, we studied whether SOBIR1 also plays a role in these processes.

We first studied whether SOBIR1 regulates the rosette leaf number of Arabidopsis plants. Consistent with the published data that CLV3 signalling negatively regulates the rosette leaf number (Clark et al., 1995), when compared with Col-0, the clv2 and crn mutants developed more rosette leaves (Fig. 3 and S1A). We also found that the sobir1 mutant developed more rosette leaves than Col-0 (Fig. 3 and S1A), indicating that SOBIR1 plays a role in regulating the rosette leaf number. Furthermore, when 


\section{Chapter 5}

compared with the clv2, crn and sobir1 single mutants, the generated clv2 sobir1 and crn sobir1 double mutants developed even more rosette leaves (Fig. 3 and S1A). This indicates that, while functioning together to regulate the rosette leaf number, both the CLV2/CRN complex and SOBIR1 might have additional interactors that also play a role in this process.
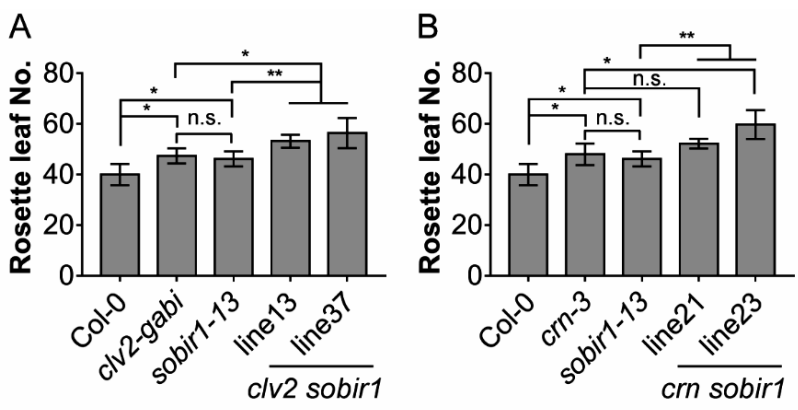

Figure 3. SOBIR1 participates in the CLV2/CRN-dependent regulation of the rosette leaf number in Arabidopsis. Six to eight plants per line were used, and rosette leaves were dissected and counted. The Student's $t$-test was used for statistical analysis, and asterisks indicate a statistically significant difference (n.s.: not significant; * $P<0.05$; ${ }^{* *} P<0.01$ ). The experiment was repeated three times with similar results, and representative results are shown.

We also studied whether SOBIR1 regulates the carpel number of siliques. Compared with Col-0, the clv2 and crn mutants developed more carpels (Fig. S1B and S1C), which is consistent with published data (Kayes and Clark, 1998; Miwa et al., 2008; Müller et al., 2008). However, similar to Col-0, the sobir1 mutant developed two carpels (Fig. S1B and S1C), indicating that SOBIR1 does not play a role in regulating carpel numbers. In addition, the clv2 sobir1 and crn sobir1 double mutants developed the same number of carpels as the clv2 and crn single mutants (Fig. S1B and S1C), suggesting that SOBIR1 is not involved in the CLV2/CRN-dependent regulation of the carpel number.

We then studied whether SOBIR1 regulates the SAM size. Consistent with the published data that CLV2 is required for CLV3-mediated termination of the SAM in Arabidopsis (Fiers et al., 2006), treatment with CLV3p caused SAM termination in Col0 , but not in the clv2 mutant (Fig. S1D). In addition, CLV3p also caused SAM termination in the sobir1 mutant, but not in the clv2 sobir1 double mutant (Fig. S1D), indicating that SOBIR1 is not involved in the CLV2-dependent CLV3-mediated 
regulation of the SAM size. We did not test SAM development in the crn mutant and the crn sobir1 double mutant upon treatment with CLV3p. However, considering the observation that SOBIR1 is not involved in the CLV2/CRN-dependent regulation of the carpel number (Fig. S1B and S1C), and also not in the CLV2-dependent CLV3mediated regulation of the SAM size (Fig. S1D), it is likely that SOBIR1 is also not involved in the CRN-dependent CLV3-mediated regulation of the SAM size.

We further studied whether SOBIR1 regulates root elongation. Consistent with the published data that CLV2 and CRN are required for suppression of root elongation triggered by CLV3p (Fiers et al., 2005; Miwa et al., 2008; Müller et al., 2008), CLV3p caused a short root phenotype in Col-0, but not in the clv2 and crn mutants (Fig. S1E and S1F). CLV3p also caused a short root phenotype in the sobir1 mutant (Fig. S1E and S1F). However, this peptide did not suppress root elongation in the clv2 sobir1 and crn sobir1 double mutants, an observation which is similar to that of the clv2 and crn single mutants (Fig. S1E and S1F). These observations indicate that SOBIR1 is not required for the CLV2/CRN-dependent response to CLV3p in the root. CLV2 and CRN are also required for suppression of root elongation triggered by CLE9p and CLE45p (Depuydt et al., 2013; Kondo et al., 2011). However, we observed that SOBIR1 is not required for the response to these CLE peptides in the root either (Fig. S1G and S1H).

Taken together, SOBIR1 participates in the CLV2/CRN-dependent regulation of the rosette leaf number in Arabidopsis, but SOBIR1 is not involved in CLV2/CRNdependent CLE signalling in the shoot and in the root.

\section{CLV2 interacts with BAK1, but this regulatory RLK appears not to be required for the response to CLV3p in the root}

Both SOBIR1 and BAK1 act as regulatory RLKs for RLPs involved in immunity (Gust and Felix, 2014; Liebrand et al., 2014; van der Burgh and Joosten, 2019; Wan et al., 2019). As CLV2 interacts with SOBIR1 (Fig. 1), we studied whether CLV2 also interacts with BAK1. Therefore, BAK1-Myc was transiently co-expressed with CLV2-eGFP or GUS-eGFP, in the presence of P19, in leaves of $N$. benthamiana. Immunoprecipitation of CLV2-eGFP, but not GUS-eGFP, resulted in co-purification of BAK1-Myc (Fig. 4A), indicating that BAK1 also interacts with CLV2 when transiently co-expressed in $N$. benthamiana.

The observed interaction between CLV2 and BAK1 indicates that CLV2 might play a role in BAK1-dependent signalling. As CLV2 regulates RLP23- and FLS2-mediated immune responses (Fig. 2B and $2 \mathrm{C}$ ) and both RLP23 and FLS2 require BAK1 for their functionality (Albert et al., 2015; Chinchilla et al., 2007; Schulze et al., 2010), CLV2 
might also regulate RLP23 and FLS2 signalling through its interaction with BAK1. As BAK1 was reported not to be required for RLP42 signalling (Zhang et al., 2014), and BAK1 and BAK1-LIKE 1 (BKK1, also known as SERK4) are the two major SERKs, with BKK1 to a lesser extent, that are redundantly required for RLP23 and FLS2 signalling (Albert et al., 2015; Roux et al., 2011), we studied whether BAK1 and BKK1 are also redundantly required for RLP42 signalling. We found that the development of BcPG3triggered necrotic symptoms was not affected in the T-DNA insertion mutant bak1-4 and the point mutant bak1-5 (Fig. S2A), both of which display defects in immunity (Roux et al., 2011; Schwessinger et al., 2011). However, this necrosis was completely suppressed in the bak1-5 bkk1-1 double mutant (Fig. S2A), indicating that, similar to their redundant roles in RLP23 and FLS2 signalling (Albert et al., 2015; Roux et al., 2011), BAK1 and BKK1 are also redundantly required for RLP42 signalling. Collectively, it is likely that CLV2 interacts with BAK1 and affects BAK1-dependent immune responses mediated by RLPS and FLS2.
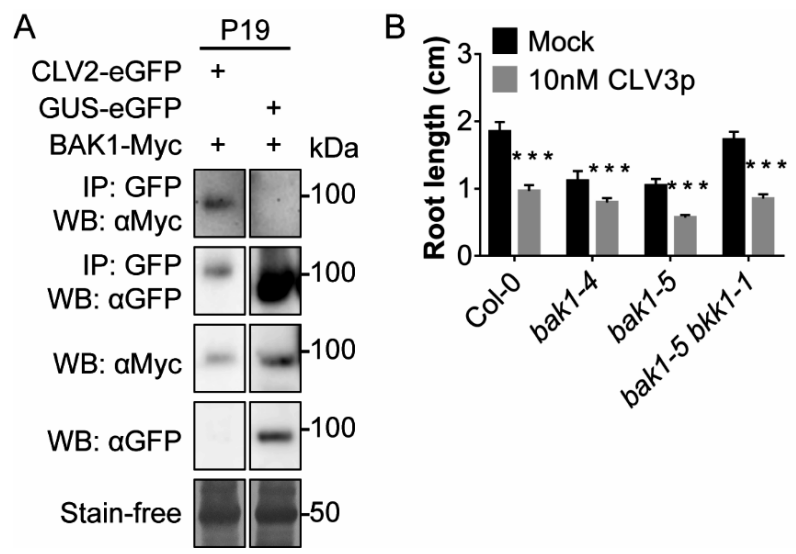

Figure 4. CLV2 interacts with BAK1, however BAK1 and its paralogue BKK1 appear not to be required for the response to CLV3p in the root. (A) CLV2 interacts with BAK1. CLV2-eGFP or GUSeGFP were co-expressed with BAK1-Myc in the presence of P19 in leaves of $N$. benthamiana. Proteins were extracted and subjected to IP by using GFP-affinity beads, followed by WB. aGFP antibody was used to detect CLV2-eGFP and GUS-eGFP, and aMyc antibody was used to detect BAK1-Myc. Rubisco bands in the stain-free panel indicate equal loading. (B) BAK1 and BKK1 appear not to be required for the response to CLV3p in the root. CLV3p was used at a final concentration of $10 \mathrm{nM}$, and MQ water was used as a control (Mock). The Student's $t$-test was used for statistical analysis, and asterisks indicate a statistically significant difference $\left({ }^{* * *} P<0.001\right)$. These experiments were repeated three times with similar results, and representative results are shown.

The interaction between BAK1 and CLV2 also suggests that BAK1 might play a role in 
CLV2-dependent CLE signalling. Therefore, we studied whether BAK1 and BKK1 are required for the CLV2-dependent response to CLV3p in the root and found that the bak1 single mutants and the bak1-5 bkk1-1 double mutant were still sensitive to CLV3p (Fig. 4B). As BAK1 and its paralogues display high functional redundancy in developmental signalling (Ma et al., 2016; Meng et al., 2015; Meng et al., 2016; Zhang et al., 2016b), we also examined serk1-1 and serk2-1 single mutants and different higher order combinations of various serk mutants for their responsiveness to CLV3p. Since serk1-1 serk2-1 double mutants are male sterile (Albrecht et al., 2005; Colcombet et al., 2005), the serk1-1 serk2-1 $1^{(+-)}$(where ${ }^{(+/)}$indicates that the indicated gene is heterozygous), serk1-1(+-) serk2-1 bak1-4 and serk1-1 serk2-1(+-) bkk1-1 mutants were used. In addition, as bak1-4 bkk1-1 double mutants display a lethal phenotype (He et al., 2007), the bak1-4(+/-) bkk1-1 and serk2-1 bak1-4(+-) bkk1-1 mutants were used. We found that the serk1-1 and serk2-1 single mutants, and the serk1-1 serk2-1 ${ }^{(+-)}$mutant were also sensitive to CLV3p (Fig. S2B). In addition, the serk1-1 bak1-4 and serk1-1 bkk1-1 double mutants were also sensitive to CLV3p (Fig. S2B). Moreover, the serk1-1 ${ }^{(+-)}$serk2-1 bak1-4, serk1-1 serk2-1(+/) bkk1-1 and serk21 bak1-4(+/-) bkk1-1 mutants were also sensitive to CLV3p (Fig. S2B). Collectively, these observations indicate that the SERKs appear not to be required for the response to CLV3p in the root.

\section{The $G \beta$ subunit $A G B 1$, and Gy subunits $A G G 1 / 2$ are required for the response to CLV3p in the root}

Although various CLV3 receptors in the SAM have been identified, downstream components required for CLV3 signalling are barely known (Somssich et al., 2016b). As the G-protein subunits AGB1 and AGG1/2 are required for CLV3-triggered signalling in the shoot and also for FLS2 signalling (Ishida et al., 2014; Liang et al., 2016; Liang et al., 2018; Wang et al., 2018a), we speculated that there might be more downstream components overlapping between the signalling pathways triggered by CLV3 receptors and FLS2. Since the root length assay allows to efficiently screen for downstream components required for CLV3 signalling, we examined Arabidopsis mutants and transformants, which display impaired FLS2 signalling, for their responsiveness to CLV3p in the root.

We first examined whether AGB1 and AGG1/2 are required for suppression of root elongation triggered by CLV3p. We found that the agb1 mutant was insensitive to treatment with CLV3p (Fig. 5A), indicating that AGB1 is also required for the response to CLV3p in the root. In addition, the agg1 agg2 double mutant, but not the agg1 and agg2 single mutants, was also insensitive to CLV3p (Fig. 5B), indicating that AGG1 and $A G G 2$ are redundantly required for the response to CLV3p in the root. 
In addition to AGB1 and AGG1/2, the Arabidopsis G-protein family also contains the Ga subunit GPA1, three XLG proteins that all contain a $\mathrm{Ga}$ domain in their C-terminus (Ding et al., 2008), and another Gy subunit referred to as AGG3 (Li et al., 2012). Except for AGG3 and XLG1, the other G-protein subunits are all required for FLS2 signalling, with XLG2 and XLG3 being redundantly required (Liang et al., 2016; Liang et al., 2018). In addition, the GTPase-accelerating protein REGULATOR OF G-PROTEIN SIGNALING 1 (RGS1) inactivates Ga subunits to stabilize the heterotrimeric G-protein complex, thereby negatively regulating FLS2 signalling (Liang et al., 2018). Arabidopsis mutants of all these different signalling components were additionally tested for their responsiveness to CLV3p. We found that the rgs 1, gpa $1, x \lg 2$ and $x \lg 3$ single mutants and the $x \lg 2 \times \lg 3$ double mutant all developed a short root phenotype upon treatment with CLV3p (Fig. S3), indicating that RGS1, GPA1, XLG2 and XLG3 are not required for the response to CLV3p in the root. Although XLG1 is not required for FLS2 signalling (Liang et al., 2016), we also tested the $x \lg 1, x \lg 1 x \lg 2 x \lg 3$ and gpa1 xlg1 xlg2 xlg3 mutants for their responsiveness to treatment with CLV3p, and found that none of these Ga-containing subunits is required for the response to CLV3p in the root (Fig. S3B). Additionally, AGG3, which requires GPA1 and AGB1 to regulate organ size and shape in Arabidopsis ( $\mathrm{Li}$ et al., 2012), is also not required for the response to CLV3p in the root (Fig. S3A). Taken together, we found that RGS1, GPA1, $X L G 2$ and $X L G 3$, which are all required for FLS2 signalling, are not required for the response to CLV3p in the root. In addition, XLG1 and AGG3 also appear not to play a role in the response to CLV3p in the root.
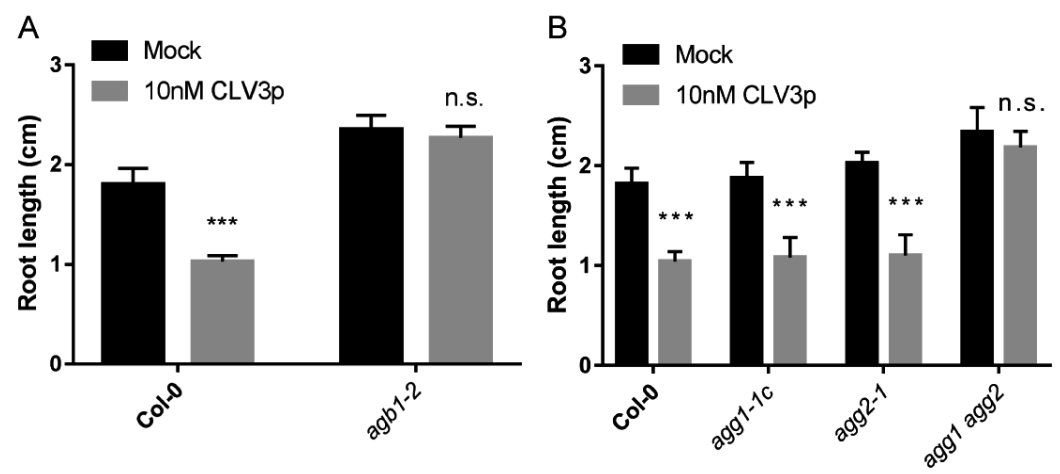

Figure 5. AGB1 and $A G G 1 / 2$ are required for the response to CLV3p in the root. (A) AGB1 is required for the response to CLV3p in the root. (B) $A G G 1$ and $A G G 2$ are redundantly required for the response to CLV3p in the root. CLV3p was used at a final concentration of $10 \mathrm{nM}$, and $M Q$ water was used as a control (Mock). The Student's $t$-test was used for statistical analysis, and asterisks indicate a statistically significant difference (n.s.: not significant; ${ }^{* * *} P<0.001$ ). These experiments were repeated three times with similar results, and representative results are shown. 


\section{Various additional regulators of FLS2 signalling are not required for the response to CLV3p in the root}

As AGB1 and AGG1/2 are involved in signalling pathways triggered by CLV3 receptors and also by FLS2 (Fig. 5) (Ishida et al., 2014; Liang et al., 2016; Liang et al., 2018; Wang et al., 2018a), we examined more Arabidopsis mutants and transformants, which display impaired FLS2 signalling, for their responsiveness to treatment with CLV3p in the root.

We found that the pseudokinases BIR2 and BIR3, which sequester BAK1 from FLS2 to negatively regulate FLS2/BAK1 complex formation (Halter et al., 2014b; Imkampe et al., 2017), are not required for the response to CLV3p in the root (Fig. S4A and S4B). In addition, BIR4, which is the closest paralogue of BIR3 (Halter et al., 2014b) and has not been functionally characterized, is also not required for this response (Fig. S4C).

The RLCK BIK1 and its paralogue AVRPPHB SUSCEPTIBLE 1-LIKE 1 (PBL1) function downstream of the FLS2/BAK1 complex and activate ROBHD to produce ROS (Li et al., 2014b; Lu et al., 2010; Zhang et al., 2010). However, BIK1, PBL1 and RBOHD all appear not to be required for the response to CLV3p in the root (Fig. S4D and S4E). In agreement with this, the protein phosphatases PP2C38 and PP2C48, which dephosphorylate BIK1 to negatively regulate FLS2 signalling (Couto et al., 2016), are also not required for the response to CLV3p in the root (Fig. S4F). Furthermore, the additional RLCKs BR-SIGNALING KINASE1 (BSK1), PATTERN-TRIGGERED IMMUNITY COMPROMISED RLCK 1 (PCRK1) and PCRK2, which all play a role in FLS2 signalling (Kong et al., 2016; Shi et al., 2013), are also not required for the response to CLV3p in the root (Fig. S4D). Additionally, BSK8 and BSK12, which are paralogues of BSK1 and have not been functionally characterized, are also not required for this response (Fig. S4D).

A typical MAPK cascade consists of three tiers of protein kinases, namely MAP kinase kinase kinases (MAPKKKs), MAP kinase kinases (MKKs) and MAP kinases (MPKs) (Meng and Zhang, 2013), and the cascade MAPKKK3/5-MKK4/5-MPK3/6 is required for FLS2 signalling (Asai et al., 2002; Bi et al., 2018). It has furthermore been reported that the MAPKKK ENHANCED DISEASE RESISTANCE 1 (EDR1) interacts with MKK4/5 to negatively regulate the activity of MPK3/6 (Geissler et al., 2015; Zhao et al., 2014), and that MPK11 negatively regulates FLS2 signalling by an unknown mechanism (Bethke et al., 2012). However, EDR1, MPK3 and MPK11 are all not required for the response to CLV3p in the root (Fig. S4G). So far, various phosphorylation substrates of MPK6 in FLS2 signalling have been identified, including the transcription factors (TFs) ETHYLENE RESPONSE FACTOR 104 (ERF104) and 
TANDEM ZINC FINGER PROTEIN 9 (TZF9), and MPK3/6-TARGETED VQP 1 (MVQ1), the latter which belongs to a class of $V Q$ motif-containing proteins and interacts with various TFs (Bethke et al., 2009; Feilner et al., 2005; Maldonado-Bonilla et al., 2013; Pecher et al., 2014). However, ERF104, TZF9 and MVQ1 are also not required for the response to CLV3p in the root (Fig. S4H).

Taken together, we found that various regulators of FLS2 signalling, as well as some paralogues of these regulators, are not required for the response to CLV $3 p$ in the root.

\section{Discussion}

\section{CLV2 interacts with both SOBIR1 and BAK1}

SOBIR1 functions as a regulatory RLK for RLPs involved in immunity (Gust and Felix, 2014; Liebrand et al., 2014; van der Burgh and Joosten, 2019; Wan et al., 2019), and this RLK employs its GxxxG motif-containing trans-membrane (TM) domain to interact with Cf-4 (Bi et al., 2016). Such a motif is also present in the TM domain of most RLPs, including Cf-4 and Cf-9, the latter which confers resistance to strains of C. fulvum secreting the effector Avr9 (Gust and Felix, 2014; Jones et al., 1994; van den Ackerveken et al., 1992). The Cf-4 and Cf-9 proteins share an identical C-terminal half, including the GxxxG motif-containing TM domain (Thomas et al., 1997). Since the GxxxG motif is required for the functionality of the Cf-9 protein (Wulff et al., 2004), it suggests that such a motif might be essential for RLPs to interact with SOBIR1. However, since some RLPs like CLV2, which do not have such a GxxxG motif in their TM domain, also interact with SOBIR1 (Fig. 1) (Gust and Felix, 2014; Liebrand et al., 2013). Collectively, these observations suggest that the GxxxG motif is dispensable for some RLPs to interact with SOBIR1. Alternatively, in addition to the GxxxG motif, the oppositely charged juxtamembrane domains of SOBIR1 and RLPs may also be responsible for their interaction (Gust and Felix, 2014).

It is known that RLPs involved in immunity recruit BAK1 in a ligand-dependent manner (Gust and Felix, 2014; Liebrand et al., 2014; van der Burgh and Joosten, 2019; Wan et al., 2019). However, this appears not to be the case for the interaction between BAK1 and RLPs involved in development. For instance, BAK1 constitutively interacts with TOO MANY MOUTHS (TMM) and RLP44, which are involved in regulation of stomatal development and plant growth, respectively (Holzwart et al., 2018; Meng et al., 2015; Nadeau and Sack, 2002; Wolf et al., 2014; Yang and Sack, 1995). Hence, the observed constitutive interaction between CLV2 and BAK1 is not unexpected (Fig. 4A). Nevertheless, since it has also been reported that CLV2 does not co-purify with BAK1 when co-expressed in Arabidopsis protoplasts (Zhu et al., 2010b), and that 
BAK1 does not co-localize with CLV2 and CRN at the plasma membrane when coexpressed in leaves of $N$. benthamiana (Bleckmann et al., 2010), further research is required to study whether indeed CLV2 interacts with BAK1 in Arabidopsis in vivo.

\section{The CLV2/CRN complex might modulate various cellular signalling processes through its interactors}

It has been demonstrated that TMM and RLP44 regulate stomatal development and plant growth, respectively (Holzwart et al., 2018; Nadeau and Sack, 2002; Wolf et al., 2014; Yang and Sack, 1995). However, instead of functioning as ligand-binding receptors, both TMM and RLP44 function as signalling modulators to regulate these developmental processes (Holzwart et al., 2018; Lee et al., 2012; Lin et al., 2017; Wolf et al., 2014). For instance, stomatal development is regulated by the secreted endogenous peptides EPIDERMAL PATTERNING FACTOR 1 (EPF1) and EPF2, which trigger the association of their receptors, RLKs of the ERECTA family (ERf), and the co-receptor BAK1 (Hara et al., 2007; Hunt and Gray, 2009; Meng et al., 2015; Shpak et al., 2005). Both the ERfs and BAK1 constitutively interact with TMM, which in its turn contributes to ERf-mediated recognition of EPF1/2 (Lee et al., 2012; Lin et al., 2017; Meng et al., 2015). Another example is the observation that RLP44 regulates plant growth through activating brassinosteroid and phytosulfokine signalling (Holzwart et al., 2018; Wolf et al., 2014), which are mediated by the RLKs BRASSINOSTEROID INSENSITIVE 1 (BRI1) and PHYTOSULFOKINE RECEPTOR 1 (PSKR1), respectively (Amano et al., 2007; Li and Chory, 1997; Matsubayashi et al., 2006; Matsubayashi et al., 2002). Both BRI1 and PSKR1 recruit BAK1 upon ligand perception (Li et al., 2002; Nam and Li, 2002; Wang et al., 2015), and RLP44 in its turn functions as a scaffold to stabilize the BRI1/BAK1 and PSKR1/BAK1 complexes (Holzwart et al., 2018; Wolf et al., 2014).

Although CLV2 is required for CLV3 signalling, CLV2 does not bind CLV3 (Shinohara and Matsubayashi, 2015). Studies have also revealed that CLV2 and CRN require each other for their re-localization from the endoplasmic reticulum to the plasma membrane, where the CLV2/CRN complex interacts with the RLKs CLV1 and BAM3, which are receptors of CLV3 and CLE45, respectively (Bleckmann et al., 2010; Hazak et al., 2017; Ogawa et al., 2008; Shinohara and Matsubayashi, 2015; Zhu et al., 2010b). In addition, CLV2 and CRN are required for the accumulation of the CLV1 and BAM3 proteins, respectively (Hazak et al., 2017; Jeong et al., 1999). Collectively, these observations suggest that CLV2 does not act as a receptor to recognize extracellular signals at the plasma membrane but forms a constitutive complex with CRN to modulate CLE signalling through regulating the accumulation of CLE receptors. In this case, since both CLV2 and CRN, as well as CLV1, are required for CLV3-mediated 
regulation of the rosette leaf number in Arabidopsis (Clark et al., 1995), it is likely that the CLV2/CRN complex regulates the accumulation of CLV1 to participate in CLV3 signalling in rosette leaves. In addition, the observed suppressive effect of SOBIR1 on the amount of rosette leaves (Fig. 3 and S1A) might be due to impaired CLV3 signalling. Since SOBIR1 facilitates the accumulation of Cf-4 (Liebrand et al., 2013), SOBIR1 probably controls the accumulation of CLV2 to indirectly participate in this developmental process. Moreover, CLV2 and CRN probably also support the accumulation of SOBIR1 and BAK1, thereby resulting in impaired SOBIR1- and/or BAK1-related immune responses in rosette leaves as observed in the clv2 and crn mutants (Fig. 2 and S2A). However, it remains unknown why SOBIR1 does not affect the CLV2/CRN-dependent CLE signalling in regulating SAM size, carpel number and root elongation (Fig. S1B to $\mathrm{S} 1 \mathrm{H}$ ), probably SOBIR1 is not co-expressed with CLV2 and $C R N$ in these plant tissues.

\section{SERKs are redundantly required for CLE signalling}

Besides functioning as regulatory proteins for RLPs and as co-receptors for RLKs in immunity, SERKs also function as co-receptors for RLKs mediating developmental processes, including the regulation of organ abscission, stomatal development and vascular development in Arabidopsis (Gust and Felix, 2014; Liebrand et al., 2014; Ma et al., 2016; Meng et al., 2015; Meng et al., 2016; van der Burgh and Joosten, 2019; Wan et al., 2019; Zhang et al., 2016b). For instance, the CLE peptide TRACHEARY ELEMENT DIFFERENTIATION INHIBITORY FACTOR (TDIF), which is identical to the CLE domain of CLE41 and CLE44, regulates vascular development through its recognition by the RLK TDIF RECEPTOR/PHLOEM INTERCALATED WITH XYLEM (TDR/PXY) and the co-receptors SERKs (Fisher and Turner, 2007; Hirakawa et al., 2008; Ito et al., 2006; Zhang et al., 2016a; Zhang et al., 2016b). While single and double mutants of the serks respond normally to TDIF, a serk1-1 serk2-1 bak1-4 triple mutant displays a moderate defect in TDIF signalling and this defect is more apparent in a serk1-1 serk2-1 bak1-5 triple mutant (Zhang et al., 2016b). In addition, the SERKs are similarly required for the regulation of organ abscission and stomatal development (Meng et al., 2015; Meng et al., 2016). These reports indicate that the various SERKs are redundantly required in developmental processes and that serk1-1 serk2-1 bak14 and serk1-1 serk2-1 bak1-5 triple mutants should be used to reveal developmental defects.

In this study, we found that the SERKs appear not to be required for the response to CLV3p in the root (Fig. S2B), which is consistent with an earlier report describing that the individual SERKs are not required for suppression of root elongation mediated by various CLE peptides (Hazak et al., 2017). However, since the serk1-1 $1^{(+-)}$serk2-1 
bak1-4 mutant used in this study still contains one functional SERK1 allele, we cannot rule out the possibility that the heterozygous allele serk $1-1^{(+/)}$may suppress a potential moderate defect of this mutant in responding to CLV3p. Therefore, serk1-1 serk2-1 bak1-4 and serk1-1 serk2-1 bak1-5 triple mutants should be further tested for their responsiveness to CLV3p in the root, in order to study whether indeed the SERKs are required for CLV3 signalling.

\section{The $G \beta$ subunit $A G B 1$ and the $G y$ subunits $A G G 1 / 2$ might function downstream of CLV2 in the Arabidopsis CLE signalling pathway}

A typical heterotrimeric G-protein complex consists of $\mathrm{Ga}, \mathrm{G} \beta$ and $\mathrm{G}$ y subunits, and is kept in an inactivated state by plasma membrane-associated GTPase-accelerating proteins such as RGS1 (Pandey, 2019), which is exemplified by the RGS1-coupled heterotrimeric G-protein complex involved in FLS2 signalling pathway (Liang et al., 2016; Liang et al., 2018). However, this is not the case for CLV3 signalling in Arabidopsis, as only the $\mathrm{G} \beta$ subunit $A G B 1$ and the $G y$ subunits $A G G 1 / 2$ are required for CLV3 signalling in the shoot (Ishida et al., 2014) and in the root (Fig. 5 and S3). In addition, the requirement of Ga-containing subunits for CLV3 signalling differs in different plant species, since GPA1 and XLGs are not required for CLV3 signalling in Arabidopsis (Ishida et al., 2014) (Fig. S3), whereas the maize (Zea mays, Zm) GPA1 orthologue COMPACT PLANT2 (CT2), which functions redundantly with ZmXLGs, interacts with the maize CLV2 orthologue FACIATED EAR2 (FEA2) to regulate CLV3 signalling (Bommert et al., 2013; Wu et al., 2018b). Furthermore, it has been recently reported that FEA2 forms distinct complexes with $Z m C R N$ and CT2 and participates in perception of different ZmCLE peptides to regulate the SAM size (Je et al., 2018). Collectively, these observations suggest a differential requirement of G-protein subunits for CLE signalling in different plant species.

In Arabidopsis, both CLV2 and the RLK RPK2 are required for CLV3 signalling (Fiers et al., 2005; Kinoshita et al., 2010), and they function redundantly with AGB1 and AGG1/2 to regulate the SAM size (Ishida et al., 2014). In this scenario, AGB1 and AGG1/2 might function downstream of either CLV2 or RPK2, or both of them. Nevertheless, AGB1 and AGG1/2 are also required for RLP23 signalling (Wan et al., 2018), which supports the notion that $A G B 1$ and $A G G 1 / 2$ function downstream of RLPs.

\section{The tested downstream components playing a role in FLS2 signalling are not required for CLV3 signalling in the root}

Although cell surface receptors recognize their respective ligands to mediate distinct signalling outputs, studies on signalling pathways triggered by different receptors have revealed various similarities for these receptors in employing downstream components. 
For instance, SERKs form ligand-induced complexes with receptors mediating immunity, such as FLS2 and Cf-4, and with receptors mediating development, such as BRI1 and TDR/PXY (Chinchilla et al., 2007; Gust and Felix, 2014; Li et al., 2002; Liebrand et al., 2014; Ma et al., 2016; Nam and Li, 2002; Postma et al., 2016; Schulze et al., 2010; van der Burgh and Joosten, 2019; Wan et al., 2019; Zhang et al., 2016b). Increasing evidence has also revealed that downstream cytoplasmic RLCKs are the direct substrates of receptor complexes, as, for example, both FLS2- and BRI1mediated signalling require the RLCKs BIK1 and BSK1 (Liang and Zhou, 2018; Lin et al., 2013b). Furthermore, the activation of downstream MAPKs is another common theme, such as the MAPK members MKK $4 / 5$ and MPK $3 / 6$ are generally required for immunity and development mediated by cell surface receptors (Meng and Zhang, 2013; Xu and Zhang, 2015; Zhang et al., 2018).

In addition to the commonalities, the establishment of specific signalling pathways, which allows to precisely respond to different extracellular stimuli, is vital for plants to maintain the homeostasis between immunity and development. So far, some determinants have been demonstrated to contribute to signalling specificity. First, receptors involved in either immunity or development have differential requirements of specific co-receptors. For instance, BAK1 and BKK1 are primarily required for receptors, like FLS2, in immunity, while BAK1 and SERK1 are more important than SERK2 and BKK1 in developmental processes mediated by receptors, such as the ERfs (Ma et al., 2016; Meng et al., 2015; Meng et al., 2016; Roux et al., 2011; Zhang et al., 2016b). In addition, CLV3-INSENSITIVE RECEPTOR KINASEs (CIKs), which are paralogues of the SERKs, function as regulatory RLKs for CLV1 and RPK2 to control CLV3 signalling in the shoot (Hu et al., 2018). It has also been shown that a different sub-localization of certain receptors and their interactors in specific nanodomains is another determinant that contributes to signalling specificity (Burkart and Stahl, 2017; Ott, 2017), as FLS2 and BRI1 localize to distinct nanodomains with BAK1 and BIK1 (Bucherl et al., 2017; Hutten et al., 2017; Somssich et al., 2015). Additionally, overlapping components involved in multiple signalling pathways can define their specific roles via the employment of differential phospho-sites, such as phosphorylation of BAK1 at residues Y403, S602, T603, S604 and S612 is required for its role in FLS2 signalling, but not in BRI1 signalling (Perraki et al., 2018; Schwessinger et al., 2011). Furthermore, strikingly, RLP44 is phosphorylated in vivo, and the phospho-site S268 is important for RLP44 to activate BRI1 signalling, but not PSKR1 signalling (Gómez et al., 2019; Wolf et al., 2014).

So far, although various CLV3 receptors in the SAM have been identified, downstream components required for CLV3 signalling are barely known (Somssich et al., 2016b). 
As the G-protein subunits AGB1 and AGG1/2 are required for both CLV3- and flg22triggered signalling (Fig. 5) (Ishida et al., 2014; Liang et al., 2016; Liang et al., 2018; Wang et al., 2018a), we speculated that more overlapping components might be present in the signalling pathways triggered by both CLV3 and flg22. However, since none of the additionally tested downstream components playing a role FLS2 signalling is required for the response to CLV3p in the root (Fig. S4), we conclude that the downstream components involved in the CLV3-triggered signalling pathway are quite different from those involved in the flg22-triggered signalling pathway.

\section{The CLV2/CRN complex forms higher order complexes with different RLKs to perform its diverse roles}

Since the RLP CLV2 was found to be required for CLV3 signalling in the SAM (Jeong et al., 1999; Kayes and Clark, 1998), efforts have been devoted to search for RLKs that can provide CLV2 with a kinase domain to initiate downstream signalling. The plasma membrane-associated kinase CRN was supposed to provide such a kinase domain, as it constitutively interacts with CLV2 and functions in the same signalling pathway as CLV2 (Bleckmann et al., 2010; Miwa et al., 2008; Müller et al., 2008; Zhu et al., 2010b). However, as CRN is a pseudokinase (Nimchuk et al., 2011), the CLV2/CRN complex does probably require additional kinase-active RLKs to activate downstream responses (Pan et al., 2016). So far, CLV2 and/or CRN have been shown to interact with the RLKs CLV1, BAM3, CIKs, SOBIR1 and BAK1 (Bleckmann et al., 2010; Hazak et al., 2017; Hu et al., 2018; Zhu et al., 2010b) (Fig. 1 and 4A). However, none of these RLKs participates in all CLV2/CRN-dependent signalling pathways, and none of them functions in the same signalling pathway as the CLV2/CRN complex (Anne et al., 2018; Bleckmann et al., 2010; Durbak and Tax, 2011; Hu et al., 2018; Zhu et al., 2010b). Therefore, we hypothesize that the CLV2/CRN complex forms higher order complexes with different RLKs in different signalling pathways to perform its diverse roles.

Here we propose a model to illustrate the diverse roles of the CLV2/CRN complex (Fig. $6)$. In this model, the CLV2/CRN complex functions as a modulator to regulate signalling mediated by different receptors, probably through regulating the accumulation of these receptors and/or their regulatory proteins. For instance, the CLV2/CRN complex employs CRN to interact with CLV1, and participates in CLV1 signalling to regulate the SAM size and the carpel number in Arabidopsis (Bleckmann et al., 2010; Durbak and Tax, 2011; Zhu et al., 2010b) (Fig. 6A). The CLV2/CRN complex also employs CRN to interact with the CIKs that are regulatory RLKs for CLV1 and RPK2, and forms higher order complexes with CLV1 and RPK2 to regulate the SAM size and the carpel number (Hu et al., 2018; Kinoshita et al., 2010) (Fig. 6A). The 
CLV2/CRN complex possibly also cooperates with RPK2 through the CIKs to regulate CLE signalling in the root (Fig. 6A), as the CLV2/CRN complex, RPK2 and the CIKs are all required for the response to multiple CLE peptides in the root (Anne et al., 2018; Fiers et al., 2005; Hu et al., 2018; Kinoshita et al., 2010; Miwa et al., 2008; Müller et al., 2008). In addition, the CLV2/CRN complex interacts with BAM3, which mediates recognition of CLE45 to regulate root length (Depuydt et al., 2013; Hazak et al., 2017) (Fig. 6B). The ClKs might also be involved in CLE45 signalling, as the ClKs are required for the response to CLE45p in the root (Anne et al., 2018; Hu et al., 2018). Moreover, the CLV2/CRN complex employs CLV2 to interact with SOBIR1 and BAK1 (Fig. 1 and 4A), thereby affecting immune responses depending on SOBIR1 and BAK1 (Fig. 2, S2A and 6C). Furthermore, SOBIR1, and probably also BAK1, participates in CLV2/CRN-dependent regulation of the rosette leaf number (Fig. 3 and 6D), a process which also requires CLV3 and CLV1 (Clark et al., 1995).

\section{Materials and Methods}

\section{Plant materials and growth conditions}

Arabidopsis thaliana (At, further referred to as Arabidopsis) plants in the Columbia-0 (Col-0) background were used in this study and are listed in Table S1. sobir1-13 (SALK_009453) (Leslie et al., 2010) and clv2-gabi (GK-686A09) (Kleinboelting et al., 2012) are T-DNA insertion mutants, and crn-3 is an EMS mutant with a point mutation at Q296 resulting in a truncated kinase domain (Somssich et al., 2016a). To generate the clv2 sobir1 and crn sobir1 double mutants, the sobir1-13 mutant was crossed to the clv2-gabi and crn-3 mutants, and homozygous double mutants were identified among the $F_{2}$ progeny. Double mutants clv2-gabi sobir1-13 were identified by PCR genotyping, and double mutants crn-3 sobir1-13 were identified by PCR genotyping followed by Afll restriction digestion of the PCR fragment for crn-3 homozygous selection (Table S2).

Arabidopsis plants were grown in soil under $12 \mathrm{~h}$ of light at $21^{\circ} \mathrm{C}$, and $12 \mathrm{~h}$ of darkness at $19^{\circ} \mathrm{C}$ in a climate chamber, with a relative humidity of $70 \%$. Nicotiana benthamiana plants were grown under $16 \mathrm{~h}$ of light at $25^{\circ} \mathrm{C}$ and $8 \mathrm{~h}$ of darkness at $21^{\circ} \mathrm{C}$, in a climate chamber, with a relative humidity of $75 \%$.

\section{Binary vectors for Agrobacterium-mediated transformation}

The coding sequence of AtCLV2 was inserted into the entry vector pENTR/D-TOPO and subsequently transferred to the binary vector pBIN-KS-35S::GWY-eGFP to generate pBIN-KS-35S::AtCLV2-eGFP. Tomato SICLV2 in the binary vector pBIN-KS35S::S/CLV2-eGFP was described previously (Liebrand et al., 2013). Binary vectors 


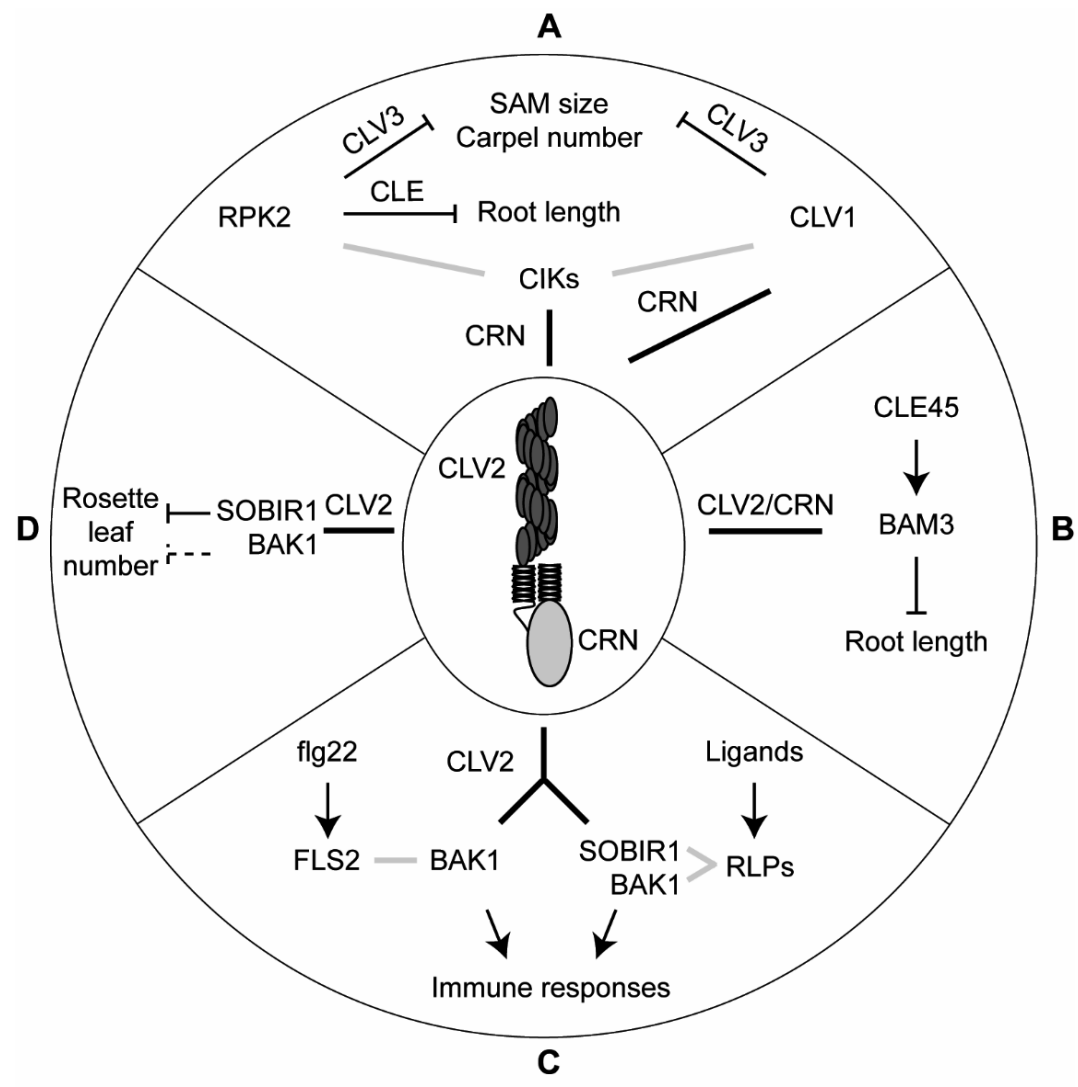

Figure 6. A model illustrating the diverse roles of the CLV2/CRN complex in immunity and development in Arabidopsis. In this model, the CLV2/CRN complex functions as a modulator to regulate signalling mediated by different receptors, probably through regulating the accumulation of these receptors and/or their regulatory proteins. (A) The CLV2/CRN complex interacts with CLV1 through CRN and participates in CLV1 signalling to regulate the SAM size and the carpel number. In addition, the CLV2/CRN complex also employs CRN to interact with the CIKs that are regulatory RLKs for CLV1 and RPK2, thereby forming higher order complexes with CLV1 and RPK2 to regulate the SAM size and the carpel number. Moreover, the CLV2/CRN complex possibly cooperates with RPK2 through the CIKs to regulate CLE signalling in the root. (B) The CLV2/CRN complex interacts with BAM3, which mediates recognition of CLE45 to regulate root length. (C) The CLV2/CRN complex employs CLV2 to interact with SOBIR1 and BAK1 and participates in SOBIR1/BAK1-dependent immune signalling mediated by RLPs, as well as in BAK1-dependent FLS2 signalling. (D) SOBIR1, and probably also BAK1, participates in the CLV2/CRN-dependent regulation of the rosette leaf number. Note that the information shown in $(A)$ and $(B)$ is based on published data (see text for further details). Bold black lines represent interactions between various RLKs and the CLV2/CRN complex through either CLV2 or CRN. Grey lines represent interactions between receptors and their regulatory proteins. The dashed line indicates an anticipated role. 
pGWB20-35S::AtSOBIR1-Myc, pGWB20-35S::SISOBIR1-Myc, pBIN-KS-35S::GUSeGFP and pBIN61-35S::P19 were described previously (Liebrand et al., 2013). Binary vectors were transformed to Agrobacterium tumefaciens strain $\mathrm{C} 58 \mathrm{C} 1$ carrying helper plasmid pCH32. Infiltration of $A$. tumefaciens suspensions into leaves was performed as described previously ( $\mathrm{Wu}$ et al., 2018a). Suspensions of the transformed Agrobacterium cells were infiltrated in leaves of $N$. benthamiana plants at a final $\mathrm{OD}_{600}$ of 1.

\section{Co-immunoprecipitations (co-IPs) and immunoblotting}

Co-IPs were performed as described previously (Liebrand et al., 2012). aGFP-HRP (Miltenyi Biotec $\mathrm{GmbH}$ ) was used to detect eGFP-tagged proteins. aMyc (Santa Cruz Biotechnology) and aMouse-HRP (GE Healthcare) were used to detect Myc-tagged proteins.

\section{BcPG3-triggered necrotic symptoms assays}

The BcPG3-triggered necrotic symptom assay was performed as described previously (Zhang et al., 2014). Briefly, BcPG3 protein was dissolved in $10 \mathrm{mM}$ sodium acetate buffer ( $\mathrm{pH} 4.2)$ to a concentration of $1.5 \mu \mathrm{M}$, and this solution was infiltrated in rosette leaves of Arabidopsis plants. Pictures were taken at 6 days post-infiltration (dpi).

\section{Reactive oxygen species (ROS) measurements}

The ROS measurement assay was performed as described (Chapter 2). flg22 and NLP24 were used at a final concentration of $100 \mathrm{nM}$ and $1 \mu \mathrm{M}$, respectively. Milli-Q (MQ) water was used as a control.

\section{Root length assays}

Arabidopsis seeds were sterilised under gaseous $\mathrm{Cl}_{2}(5 \mathrm{~mL} 37 \% \mathrm{HCl}, 50 \mathrm{~mL} \mathrm{NaClO}$ and $50 \mathrm{~mL}$ of $\mathrm{MQ}$ ) for $4 \mathrm{~h}$ and grown on $1 / 2$ Murashige \& Skoog (Duchefa Biochemie) plates. Different peptides were used as indicated, and $M Q$ water was used as a control. Thirty sterile seeds per line were used for the root length assay. The plates were coldtreated at $4^{\circ} \mathrm{C}$ in the dark for 3 days and subsequently transferred to a climate chamber with settings as mentioned above. Photos were taken at 7 days after germination (dag), and the root length was measured by Image $\mathrm{J}$.

\section{CLV3p-triggered shoot apical meristem (SAM) termination assays}

Arabidopsis seeds were prepared as the root length assay. CLV3 peptide was used at a final concentration of $10 \mu \mathrm{M}$, and $\mathrm{MQ}$ water was used as a control. Thirty sterile seeds per line were used. Photos were taken at about 20 dag. 


\section{Acknowledgements}

J.W. is supported by a PhD scholarship from the China Scholarship Council (CSC) and G.W. is supported by the 100-Talent Program of Shaanxi Province, the National Natural Science Foundation of China (31771556) and the Fundamental Research Funds for the Central Universities (GK201702016). We are highly indebted to C. Zipfel, J-M Zhou, J. van Kan, R. Simon, L. Shan, R. Panstruga, S. Assmann, Y. Li, Y. Zhang, B. Kemmerling, F. Tax, D. Tang and J. Lee for their kindness in offering us seeds of the various Arabidopsis mutants and transformants employed in this study. 


\section{Supplementary information}
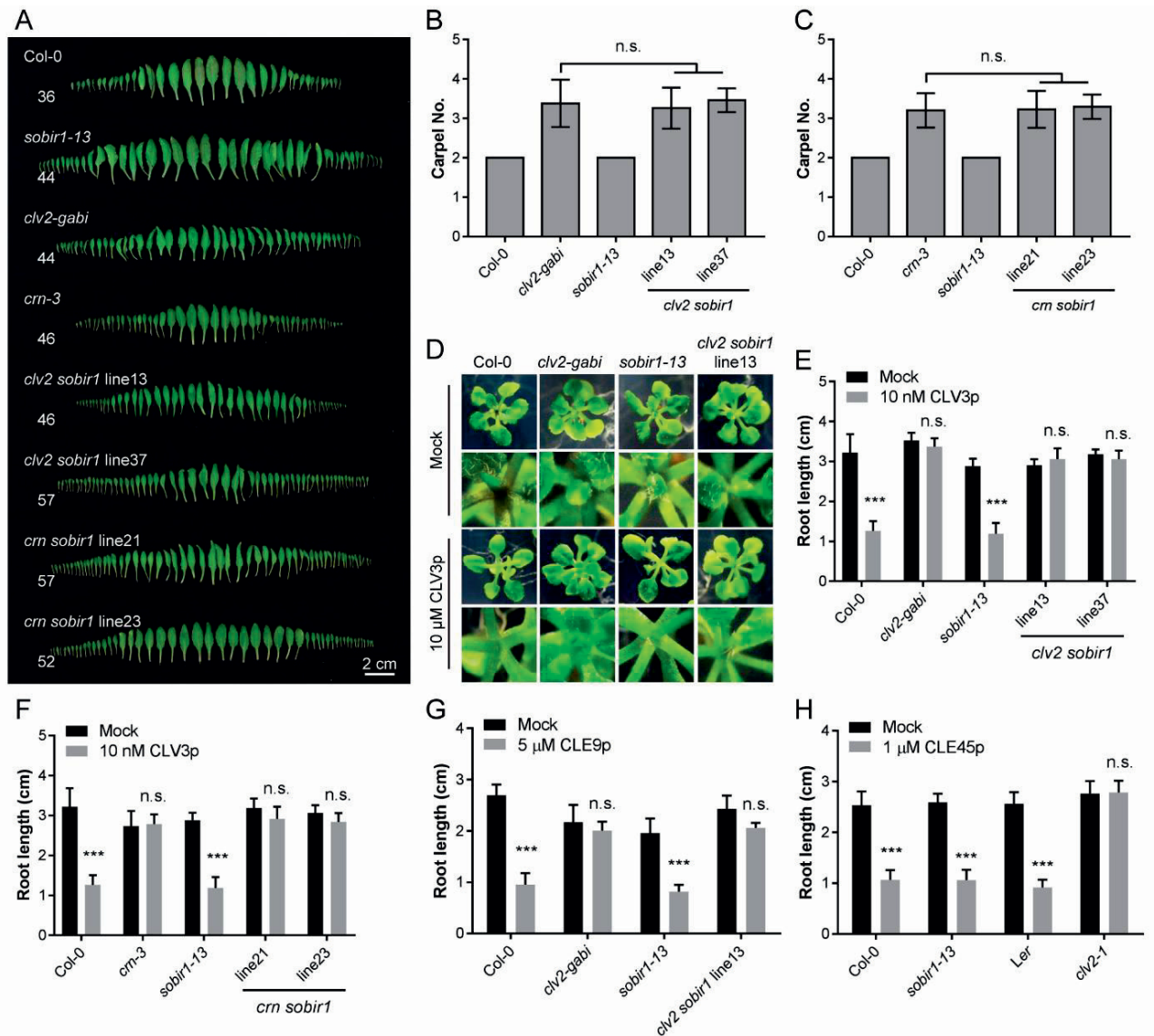

Figure S1. SOBIR1 is not required for CLE signalling in the shoot and in the root. (A) Dissected rosette leaves from the indicated Arabidopsis plants with their corresponding leaf numbers. (B, C) SOBIR1 is not involved in the CLV2/CRN-dependent regulation of the carpel number. Twenty to thirty plants per line were used, and siliques from the primary stem were used for carpel number counting. (D) SOBIR1 is not required for the CLV2-dependent SAM termination triggered by CLV3p. CLV3p was used at a final concentration of $10 \mu \mathrm{M}$. (E, F) SOBIR1 is not required for the CLV2/CRN-dependent response to CLV3p in the root. CLV3p was used at a final concentration of $10 \mathrm{nM}$. (G, H) SOBIR1 is not required for the response to CLE9p and CLE45p in the root. CLE9p and CLE45p were used at a final concentration of $5 \mu \mathrm{M}$ and $1 \mu \mathrm{M}$, respectively. Note that a different loss-of-function mutant clv2-1, which is an EMS mutant in the Ler background with a point mutation at Q33 resulting in a premature stop codon, was used for treatment with CLE45p. (D-H) MQ water was used as a control (Mock). The Student's $t$-test was used for statistical analysis, and asterisks indicate a statistically significant difference (n.s.: not significant; ${ }^{* * *} P<0.001$ ). These experiments were repeated three times with similar results, and representative results are shown. 

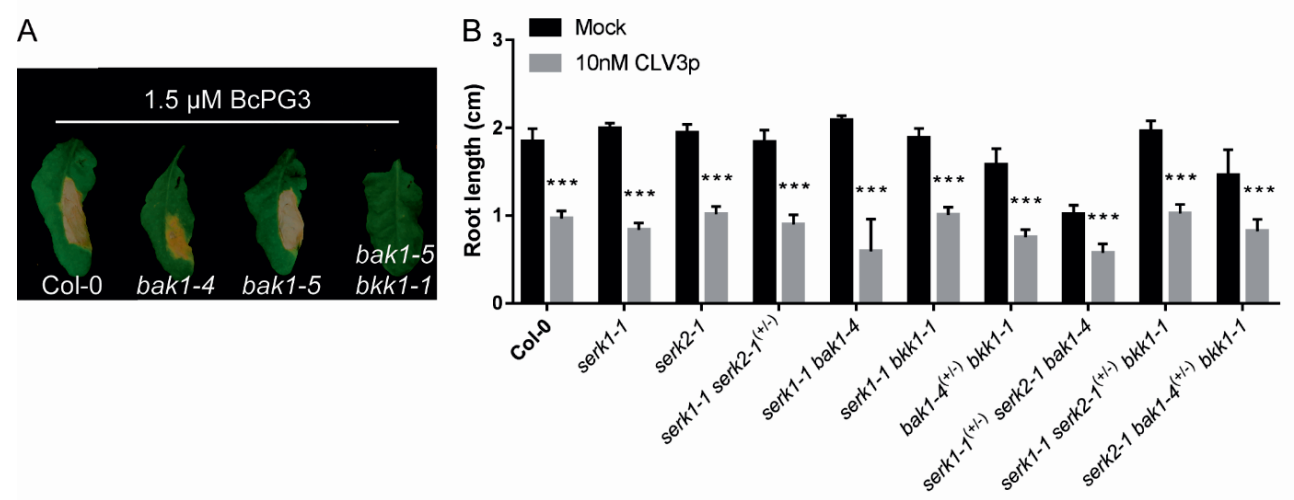

Figure S2. BAK1 and BKK1 are redundantly required for the development of BcPG3-triggered necrotic symptoms, but BAK1 and BKK1, as well as their paralogues, appear not to be required for the response to CLV3p in the root. (A) BAK1 and BKK1 are redundantly required for the development of BcPG3-triggered necrotic symptoms. Six rosette leaves from two plants of each line were infiltrated with a $1.5 \mu \mathrm{M} \mathrm{BcPG} 3$ protein solution, and pictures were taken at $6 \mathrm{dpi}$. (B) SERKs appear not to be required for the response to CLV3p in the root. CLV3p was used at a final concentration of $10 \mathrm{nM}$, and MQ water was used as a control (Mock). ${ }^{(+)}$indicates the indicated gene is heterozygous. The Student's $t$-test was used for statistical analysis, and asterisks indicate a statistically significant difference $\left({ }^{* \star *} P<0.001\right)$. These experiments were repeated three times with similar results, and representative results are shown.
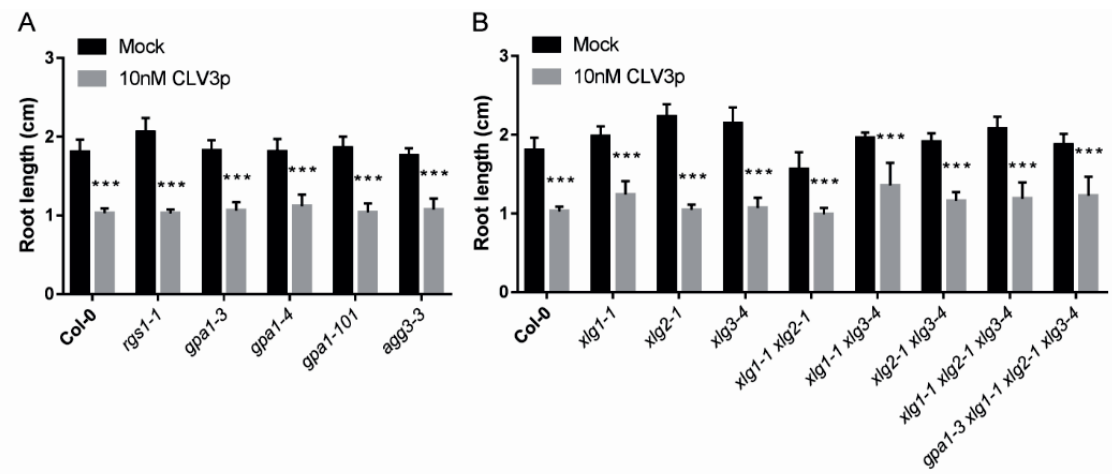

Figure S3. RGS1 and the G-protein subunits GPA1, AGG3 and the XLGs are not required for the response to CLV3p in the root. (A) RGS1, GPA1 and AGG3 are not required for the response to CLV3p in the root. (B) The XLGs, as well as GPA1, are not required for the response to CLV3p in the root. CLV3p was used at a final concentration of $10 \mathrm{nM}$, and $M Q$ water was used as a control (Mock). The Student's $t$-test was used for statistical analysis, and asterisks indicate a statistically significant difference $\left({ }^{* * *} P<0.001\right)$. These experiments were repeated three times with similar results, and representative results are shown. 

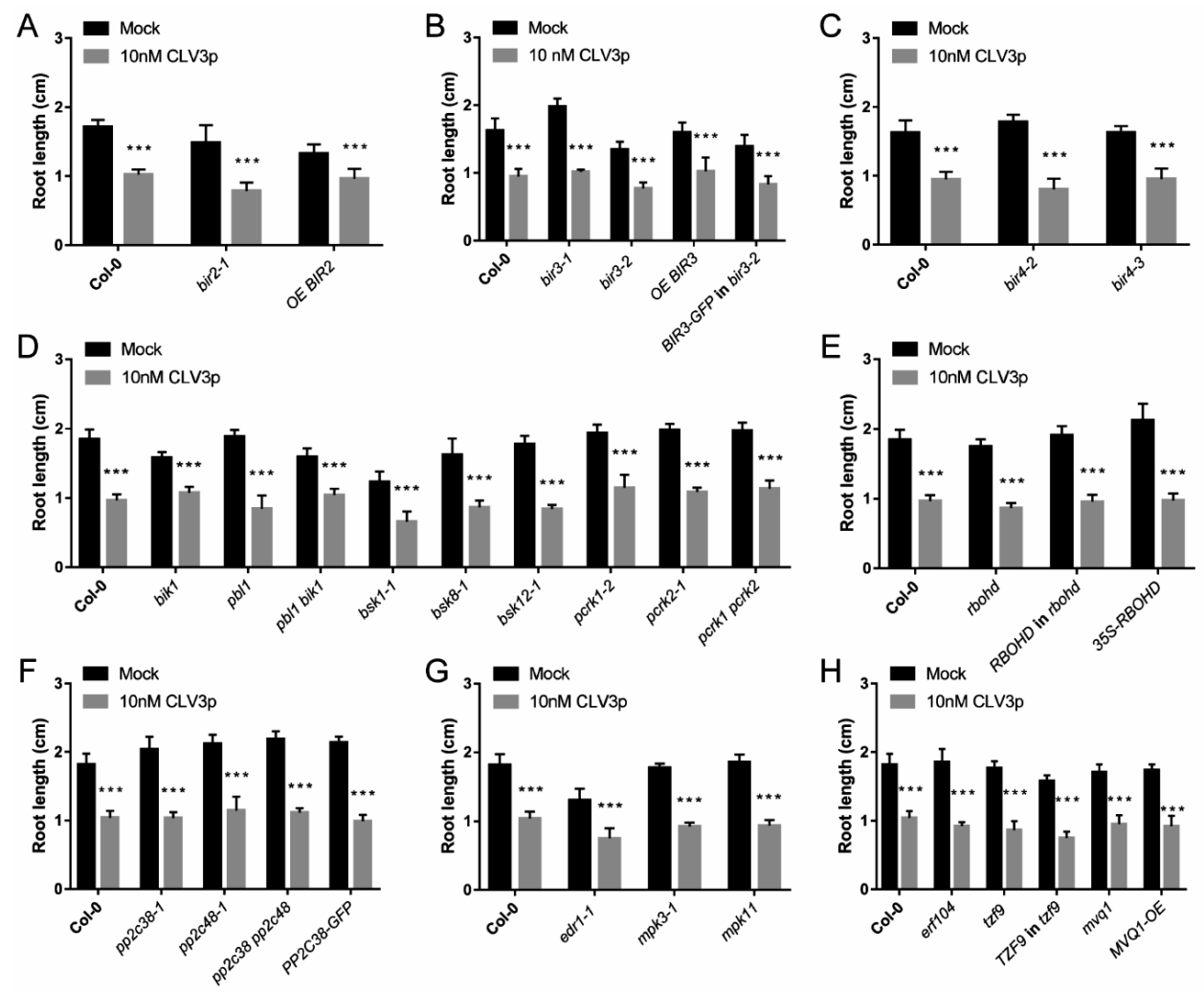

Figure S4. None of the tested FLS2 signalling regulators or their paralogues is required for the response to CLV3p in the root. (A-C) The pseudokinases BIR2 (A), BIR3 (B) and BIR4 (C) are not required for the response to CLV3p in the root. Note that BIR2 and BIR3 interact with BAK1 to negatively regulate FLS2 signalling, and that BIR4 has not been functionally characterized. (D) Various RLCKs are not required for the response to CLV3p in the root. Note that BSK1 interacts with FLS2, and that the BSK1 paralogues, BSK8 and BSK12, have not been functionally characterized. (E) RBOHD is not required for the response to CLV3p in the root. (F) The protein phosphatases PP2C38 and PP2C48 are not required for the response to CLV3p in the root. (G) The MAPKKK EDR1 and the MAPKs MPK3 and MPK11 are not required for the response to CLV3p in the root. (H) ERF104, TZF9 and MVQ1 are also not required for the response to CLV3p in the root. Note that ERF104, TZF9 and MVQ1 are all phosphorylation substrates of MPK6 in FLS2 signalling. CLV3p was used at a final concentration of 10 $\mathrm{nM}$, and MQ water was used as a control (Mock). The Student's $t$-test was used for statistical analysis, and asterisks indicate a statistically significant difference $\left.{ }^{* * *} P<0.001\right)$. These experiments were repeated three times with similar results, and representative results are shown. 
CLV2-containing complexes and the CLV3 signalling pathway

Table S1. Arabidopsis plants used in this study

\begin{tabular}{|c|c|c|}
\hline Name & Background & Reference \\
\hline Col-0 & - & - \\
\hline Ler & - & - \\
\hline clv2-gabi & Col-0 & Kleinboelting et al., 2012 \\
\hline clv2-1 & Ler & Jeong et al., 1999 \\
\hline$c r n-3$ & Col-0 & Somessich et al., 2016a \\
\hline sobir1-13 & Col-0 & Gao et al., 2009 \\
\hline bak1-4 & Col-0 & Meng et al., 2015 \\
\hline bak1-5 & Col-0 & Schwessinger et al., 2011 \\
\hline bak1-5 bkk1-1 & Col-0 & Schwessinger et al., 2011 \\
\hline serk1-1 & Col-0 & Meng et al., 2015 \\
\hline serk2-1 & Col-0 & Meng et al., 2015 \\
\hline serk1-1 serk2-1 $1^{(+-)}$ & Col-0 & Meng et al., 2015 \\
\hline serk1-1 bak1-4 & Col-0 & Meng et al., 2015 \\
\hline serk1-1 bkk1-1 & Col-0 & Meng et al., 2015 \\
\hline bak1-4 $4^{(+-)}$bkk1-1 & Col-0 & Meng et al., 2015 \\
\hline serk1-1 ${ }^{(+-)}$serk2-1 bak1-4 & Col-0 & Meng et al., 2015 \\
\hline serk1-1 serk2-1 $1^{(+-)}$bkk1-1 & Col-0 & Meng et al., 2015 \\
\hline serk2-1 bak1-4 ${ }^{(+/-)}$bkk1-1 & Col-0 & Meng et al., 2015 \\
\hline$a g b 1-2$ & Col-0 & Lorek et al., 2013 \\
\hline $\operatorname{agg} 1-1 c$ & Col-0 & Liang et al., 2016 \\
\hline agg2-1 & Col-0 & Liang et al., 2016 \\
\hline $\operatorname{agg} 1$ agg2 & Col-0 & Liang et al., 2016 \\
\hline rgs $1-1$ & Col-0 & Lorek et al., 2013 \\
\hline gpa1-3 & Col-0 & Ding et al., 2007 \\
\hline gpa1-4 & Col-0 & Li et al., 2012 \\
\hline gpa1-101 & Col-0 & Li et al., 2012 \\
\hline $\operatorname{agg3-3}$ & Col-0 & Li et al., 2012 \\
\hline$x \lg 1-1$ & Col-0 & Ding et al., 2007 \\
\hline$x \lg 2-1$ & Col-0 & Ding et al., 2007 \\
\hline$x \lg 3-4$ & Col-0 & Ding et al., 2007 \\
\hline$x \lg 1-1 \times \lg 2-1$ & Col-0 & Ding et al., 2007 \\
\hline$x \lg 1-1 \times \lg 3-4$ & Col-0 & Ding et al., 2007 \\
\hline$x \lg 2-1 x \lg 3-4$ & Col-0 & Ding et al., 2007 \\
\hline$x \lg 1-1 x \lg 2-1 x \lg 3-4$ & Col-0 & Ding et al., 2007 \\
\hline gpa1-3 xlg1-1 xlg2-1 x/g3-4 & Col-0 & Ding et al., 2007 \\
\hline bir2-1 & Col-0 & Halter et al., 2014 \\
\hline OE BIR2 & Col-0 & Halter et al., 2014 \\
\hline bir3-1 & Col-0 & Imkampe et al., 2017 \\
\hline bir3-2 & Col-0 & Imkampe et al., 2017 \\
\hline OE BIR3 & Col-0 & Imkampe et al., 2017 \\
\hline BIR3-GFP in bir3-2 & Col-0 & Imkampe et al., 2017 \\
\hline bir4-2 & Col-0 & Wierzba Michael 2014 \\
\hline bir4-3 & Col-0 & Wierzba Michael 2014 \\
\hline bik1 & Col-0 & Zhang et al., 2010 \\
\hline pbl1 & Col-0 & Zhang et al., 2010 \\
\hline bik $p b / 1$ & Col-0 & Zhang et al., 2010 \\
\hline bsk1-1 & Col-0 & Shi et al., 2013 \\
\hline bsk8-1 & Col-0 & Shi et al., 2013 \\
\hline bsk12-1 & Col-0 & Shi et al., 2013 \\
\hline pcrk1-2 & Col-0 & Kong et al., 2016 \\
\hline pcrk2-1 & Col-0 & Kong et al., 2016 \\
\hline pcrk1 pcrk2 & Col-0 & Kong et al., 2016 \\
\hline rbohd & Col-0 & Kadota et al., 2014 \\
\hline$R B O H D$ in rbohd & Col-0 & Kadota et al., 2014 \\
\hline 35S:RBOHD & Col-0 & Kadota et al., 2014 \\
\hline pp2c38-1 & Col-0 & Couto et al., 2016 \\
\hline$p p 2 c 48-1$ & Col-0 & Couto et al., 2016 \\
\hline$p p 2 c 38 p p 2 c 48$ & Col-0 & Couto et al., 2016 \\
\hline PP2C38-GFP & Col-0 & Couto et al., 2016 \\
\hline edr1-1 & Col-0 & Geissler et al., 2015 \\
\hline$m p k 3-1$ & Col-0 & Bethke et al., 2012 \\
\hline mpk11 & Col-0 & Bethke et al., 2012 \\
\hline erf104 & Col-0 & Bethke et al., 2009 \\
\hline tzfg & Col-0 & Maldonado-Bonilla et al., 2013 \\
\hline TZF9 in tzf9 & Col-0 & Maldonado-Bonilla et al., 2013 \\
\hline$m v q 1$ & Col-0 & Pecher et al., 2014 \\
\hline MVQ1-OE & Col-0 & Pecher et al., 2014 \\
\hline
\end{tabular}




\section{Chapter 5}

Table S2. Primers used for generating the clv2 sobir1 and crn sobir1 double mutants

\begin{tabular}{ll}
\hline Primer name & Sequence \\
\hline sobir1-13-LP & 5'-TGCTGGATGGAAAGTGGTATC-3' \\
sobir1-13-RP & 5'-ACCCACGAATCATTCACAGAG-3' \\
LBb1.3 & 5'-ATTTTGCCGATTTCGGAAC-3' \\
clv2-gabi-LP & 5'-CGGATTACGTACTGAATCAAACC-3' \\
clv2-gabi-RP & 5'-ACTAAGAGACGGACGAGAGGC-3' \\
LBb Gabi & 5'-ATATTGACCATCATACTCATTGC-3' \\
crn-3-F & 5'-TGTTGGATTCTGAGTTTGAGC-3' \\
crn-3-R & 5'-TGATATTTGAAGCACCTGTTACCTT-3' \\
\hline
\end{tabular}


Chapter 6

General discussion 


\section{Introduction}

Plants rely on cell surface receptors to recognize extracellular signals to activate downstream signalling in the regulation of defence and development. Cell surface receptors are either receptor-like kinases (RLKs) that contain a ligand-binding ectodomain, a transmembrane domain and an intracellular kinase domain, or receptorlike proteins (RLPs) that are structurally similar to RLKs but only contain a short cytoplasmic tail (Monaghan and Zipfel, 2012). According to the motifs present in their ectodomains, cell surface receptors can be subdivided into different groups, with RLKs and RLPs containing leucine-rich repeat (LRR) motifs forming the largest group of cell surface receptors (Böhm et al., 2014a; Boutrot and Zipfel, 2017; Li et al., 2016c; Ranf, 2017; Saijo et al., 2018).

So far, various LRR-RLKs and LRR-RLPs have been demonstrated to play roles in defence and development (Boutrot and Zipfel, 2017; Jamieson et al., 2018; Li et al., 2016c; Ma et al., 2016; van der Burgh and Joosten, 2019; Wan et al., 2019; Wang et al., 2010a). Increasing evidence has revealed that both LRR-RLKs and LRR-RLPs acting as ligand-binding receptors require regulatory LRR-RLKs for their functionality, and some regulatory LRR-RLKs can even act as co-receptors since they also directly bind extracellular ligands (Gust and Felix, 2014; Hohmann et al., 2017; Liebrand et al., 2014; Ma et al., 2016; Song et al., 2017a; van der Burgh and Joosten, 2019; Wan et al., 2019). Ligand perception triggers the formation and activation of receptor complexes, which subsequently phosphorylate downstream signalling components, such as receptor-like cytoplasmic kinases (RLCKs), leading to the activation of a series of intracellular responses, including the activation of mitogen-activated protein kinase (MAPK) cascades (Couto and Zipfel, 2016; Cui et al., 2018; Liang and Zhou, 2018; Zhang et al., 2018; Zhou et al., 2019). In addition to positive regulators required for signal transduction, negative regulators have also been reported to prevent deregulated signalling activation in the absence of ligand perception, or to control the duration and amplitude of the activated cellular responses (Couto and Zipfel, 2016; Mithoe and Menke, 2018; Tang et al., 2017). This chapter provides an overview of the various regulatory proteins required for the functionality of LRR-type cell surface receptors, with a focus on those controlling the formation, activation and abundance of cell surface receptor complexes. The objective of this chapter is to place the findings of this thesis in a broader perspective, based on results obtained from recent studies on the regulation of LRR-type cell surface receptor complexes involved in defence and development.

\section{LRR-type cell surface receptors involved in defence and development LRR-RLKs and LRR-RLPs involved in defence}


Cell surface receptors recognize extracellular immunogenic patterns (ExIPs) to initiate extracellularly-triggered immunity (ExTI) (van der Burgh and Joosten, 2019). An ExIP can be any ExTI-triggering extracellular molecule, including microbe-associated molecular patterns (MAMPs), host-derived extracellular danger signals and microbial extracellular effectors (Boller and Felix, 2009; Cook et al., 2015; Dodds and Rathjen, 2010; Gust et al., 2017; Jones and Dangl, 2006; Kanyuka and Rudd, 2019; Thomma et al., 2011; van der Burgh and Joosten, 2019). To successfully colonize host cells, plant pathogens secrete effectors to suppress ExTI, whereas the effectors translocated into the host cells can be recognized by intracellular receptors, which are mainly nucleotide-binding leucine-rich repeat (NB-LRR) proteins (Dodds and Rathjen, 2010; Jones and Dangl, 2006; van der Burgh and Joosten, 2019). Activation of intracellular receptors leads to intracellularly-triggered immunity $(\operatorname{InTI})$, which is normally associated with the development of a hypersensitive response (HR) (Dodds and Rathjen, 2010; Jones and Dangl, 2006; van der Burgh and Joosten, 2019). ExTI typically is not associated with an HR, although some cell surface receptors do cause an HR upon recognition of their matching ExIP (Dodds and Rathjen, 2010; Jones and Dangl, 2006; van der Burgh and Joosten, 2019). However, in principle, both ExTI and $\mathrm{InTI}$ are sufficient to provide resistance to pathogens (van der Burgh and Joosten, 2019).

So far, many LRR-RLKs have been demonstrated to mediate plant defence (Boutrot and Zipfel, 2017; van der Burgh and Joosten, 2019; Wan et al., 2019). FLAGELLINSENSING 2 (FLS2) is the most well-characterized LRR-RLK and recognizes bacterial flagellin to mediate defence against infection by bacterial pathogens in various plant species, including Arabidopsis thaliana (further referred to as Arabidopsis), Nicotiana benthamiana, tomato (Solanum lycopersicum, SI) and rice (Oryza sativa) (Chinchilla et al., 2006; Felix et al., 1999; Gómez-Gómez and Boller, 2000; Hann and Rathjen, 2007; Robatzek et al., 2007; Takai et al., 2008; Zipfel et al., 2004). A structural study revealed that flg22, which is a 22-amino-acid immunogenic peptide of flagellin, directly binds to the ectodomain of FLS2 and triggers the activation of downstream immune responses (Sun et al., 2013b). EFR is another well-known RLK that recognizes the bacterial elongation factor-Tu (EF-Tu) epitope elf18 to mediate defence against bacterial infection in Brassicaceae (Zipfel et al., 2006). Additionally, the 23-amino-acid peptide PROPEP1 (Pep1), which is an endogenously secreted signal in Arabidopsis, is directly recognized by the RLK PROPEP1 RECEPTOR 1 (PEPR1) and PEPR2 to activate defence (Krol et al., 2010; Tang et al., 2015; Yamaguchi et al., 2010; Yamaguchi et al., 2006). Furthermore, the rice RLK XA21 confers resistance to the bacterial pathogen Xanthomonas oryzae pv. oryzae upon recognition of the 21-aminoacid peptide RaxX21 derived from REQUIRED FOR ACTIVATION OF XA21 (RaxX) 
(Pruitt et al., 2015; Song et al., 1995). Besides the receptors mentioned above, many additional LRR-RLKs have also been demonstrated to mediate defence in Arabidopsis and tomato (Boutrot and Zipfel, 2017; van der Burgh and Joosten, 2019; Wan et al., 2019).

Similar to LRR-RLKs, LRR-RLPs can also function as receptors to mediate plant defence (Boutrot and Zipfel, 2017). For instance, the tomato RLPs Cf-4 and Cf-9 confer resistance to strains of the fungal pathogen Cladosporium fulvum that secrete the cysteine-rich effectors referred to as avirulence factor 4 (Avr4) and Avr9, respectively (Jones et al., 1994; Joosten et al., 1994; Thomas et al., 1997; van den Ackerveken et al., 1992; van Kan et al., 1991). In N. benthamiana, XYLOGLUCAN-SPECIFIC ENDOGLUCANASE (XEG1), a MAMP derived from the oomycete pathogen Phytophthora sojae, triggers the development of an HR upon its recognition by the RLP RESPONSE TO XEG1 (RXEG1) (Ma et al., 2015; Wang et al., 2018c). Additionally, Arabidopsis RLP23 mediates defence against infection by pathogens producing NECROSIS- AND ETHYLENE-INDUCING PROTEIN 1 (NEP1)-like proteins (NLPs) (Albert et al., 2015; Böhm et al., 2014b; Oome et al., 2014). However, many additional LRR-RLPs have been demonstrated to mediate defence in tomato and Arabidopsis as well (Boutrot and Zipfel, 2017; van der Burgh and Joosten, 2019; Wan et al., 2019).

\section{LRR-RLKs and LRR-RLPs involved in development}

Many Arabidopsis RLKs have been demonstrated to directly bind endogenously secreted peptides to regulate various developmental processes (Hohmann et al., 2017; Ma et al., 2016; Shinohara and Matsubayashi, 2015; Song et al., 2017a). For instance, the RLK BRASSINOSTEROID INSENSITIVE1 (BRI1) directly recognizes brassinolide $(B L)$, which is the most active form of the brassinosteroids, to regulate plant growth (Hothorn et al., 2011; Li and Chory, 1997; She et al., 2011). In addition, the RLK PHYTOSULFOKINE RECEPTOR1 (PSKR1) directly recognizes PHYTOSULFOKINE (PSK) to regulate multiple developmental processes (Amano et al., 2007; Matsubayashi, 2014; Matsubayashi et al., 2002; Wang et al., 2015). Moreover, the RLK HAESA (HAE) directly recognizes INFLORESCENCE DEFICIENT IN ABSCISSION (IDA) to promote organ abscission (Butenko et al., 2003; Cho et al., 2008; Santiago et al., 2016), while RLKs of the ERECTA family (ERfs) directly recognize EPIDERMAL PATTERNING FACTOR1 (EPF1) and EPF2 to regulate stomatal development (Hara et al., 2007; Hunt and Gray, 2009; Lin et al., 2017; Shpak et al., 2005). Furthermore, the RLK CLAVATA1 (CLV1) directly recognizes the stem cell-secreted peptide CLV3 to regulate the activity of the shoot apical meristem (SAM) (Clark et al., 1993; Clark et al., 1995; Clark et al., 1997; Fletcher et al., 1999; Ogawa et al., 2008; Shinohara and Matsubayashi, 2015). CLV3 belongs to the CLV3/ENDOSPERM SURROUNDING 
REGION (ESR)-related (CLE) family, consisting of 32 members in Arabidopsis (Jun et al., 2008). CLE45 is recognized by the RLK BARELY ANY MERISTEM 3 (BAM3) to regulate root elongation (Depuydt et al., 2013; Hazak et al., 2017), while the CLE member TRACHEARY ELEMENT DIFFERENTIATION INHIBITORY FACTOR (TDIF) is directly recognized by the RLK TDIF RECEPTOR/PHLOEM INTERCALATED WITH XYLEM (TDR/PXY, further referred to as TDR) to regulate vascular development (Fisher and Turner, 2007; Hirakawa et al., 2008; Ito et al., 2006; Zhang et al., 2016a).

So far, only a few Arabidopsis RLPs and some of their related orthologues have been reported to play a role in development (Jamieson et al., 2018; Wang et al., 2010a; Wu et al., 2016a). For instance, the RLP CLV2 is involved in the regulation of SAM growth and root elongation mediated by CLV3 and CLE45, respectively (Depuydt et al., 2013; Hazak et al., 2017; Jeong et al., 1999; Kayes and Clark, 1998). In addition, the RLP TOO MANY MOUTHS (TMM) is involved in EPF1/2-mediated regulation of stomatal development (Nadeau and Sack, 2002; Yang and Sack, 1995). Recently, it was reported that RLP44 is involved in the regulation of cell wall integrity and vascular development through activating BRI1 and PSKR1 signalling (Holzwart et al., 2018; Wolf et al., 2014). However, it is worth noting that these three LRR-RLPs do not function as receptors but appear to act as regulatory proteins of LRR-RLK receptor complexes involved in development (see below).

\section{Complex formation of LRR-type cell surface receptors with their regulatory LRR- RLKs}

The somatic embryogenesis receptor kinase (SERK) family consists of five members, namely SERK1, SERK2, SERK3/BAK1 (BRI1-ASSOCIATED RECEPTOR KINASE 1), SERK4/BKK1 (BAK1-LIKE 1) and SERK5, which all contain five LRRs in their ectodomain (He et al., 2007). SERK5 contains a mutation in the arginine-aspartate (RD) motif of its kinase domain, which abolishes its function in BRI1 signalling (He et al., 2007; Wu et al., 2015). BAK1 and the related SERK homologues form ligandinduced complexes with LRR-RLK receptors involved in defence and development, as well as with LRR-RLP receptors involved in defence, leading to the notion that the SERKs function as regulatory RLKs for LRR-type receptors (Gust and Felix, 2014; Liebrand et al., 2014; Ma et al., 2016; van der Burgh and Joosten, 2019; Wan et al., 2019). For instance, BAK1 forms ligand-induced complexes with the RLKs FLS2, EFR, PEPR1/2, BRI1, PSKR1, HAE, the ERfs and TDR (Chinchilla et al., 2007; He et al., 2007; Ma et al., 2016; Meng et al., 2015; Meng et al., 2016; Roux et al., 2011; Tang et al., 2015; Wang et al., 2015; Zhang et al., 2016b), and the RLPs Cf-4, RXEG1 and RLP23 (Albert et al., 2015; Postma et al., 2016; Wang et al., 2018c) (Fig. 1). BAK1 also acts as a co-receptor for FLS2, since the ectodomains of FLS2 and BAK1 directly 
bind flg22, which triggers and stabilizes the heterodimerization of the ectodomains of FLS2 and BAK1 (Sun et al., 2013b). A similar molecular mechanism also holds for the heterodimerization of BAK1 with BRI1, HAE and TDR through their ectodomains (Santiago et al., 2016; Santiago et al., 2013; Sun et al., 2013a; Zhang et al., 2016b). The heterodimerization of PSKR1 and BAK1 is structurally similar to the RLK/BAK1 complexes mentioned above, except that BAK1 does not directly bind PSKR1-bound PSK (Hohmann et al., 2017; Song et al., 2017a; Wang et al., 2015). So far, it remains unknown whether the various SERKs heterodimerize with RLPs through their ectodomains.

Recently, CIK1 (CLAVATA3 INSENSITIVE RECEPTOR KINASE 1), CIK2, CIK3 and CIK4 were reported to be redundantly required for various CLE-regulated developmental processes, including the regulation of the SAM mediated by CLV3 and its receptor CLV1 (Anne et al., 2018; Hu et al., 2018). The CIKs are paralogues of the SERKs, and also contain five LRRs in their ectodomain (Hu et al., 2018). Unlike the SERKs that form a ligand-induced complex with cell surface receptors, the CIKs constitutively interact with CLV1 in a ligand-independent manner (Hu et al., 2018). So far, it remains elusive whether the CIKs heterodimerize with CLV1 through their ectodomains and play a role in SERK-dependent signalling.

Besides forming a ligand-induced complex with the SERKs, RLPs involved in defence also constitutively interact with the regulatory RLK SUPPRESSOR OF BIR1-1 (SOBIR1) (Gust and Felix, 2014; Liebrand et al., 2014; van der Burgh and Joosten, 2019; Wan et al., 2019). For instance, SOBIR1 constitutively interacts with the RLPs Cf-4, RXEG1 and RLP23 (Albert et al., 2015; Liebrand et al., 2013; Wang et al., 2018c) (Fig. 1). SOBIR1 also contains five LRRs in its ectodomain (Hohmann and Hothorn, 2019). However, the ectodomain of SOBIR 1 is not required for its constitutive interaction with Cf-4 but is required for its role in Cf-4 signalling (Bi et al., 2016). So far, the contribution of the ectodomain of SOBIR1 to its role in RLP-mediated immune signalling remains unclear, and whether the regulatory RLK SOBIR1 also functions as a co-receptor for RLPs remains to be elucidated.

In addition to participating in plant defence mediated by RLPs, SOBIR1 also plays a role in development mediated by RLKs (Leslie et al., 2010; Milhinhos et al., 2019). It was shown that SOBIR1, which is also known as EVERSHED, functions as a negative regulator in controlling organ abscission (Leslie et al., 2010). As introduced above, organ abscission is mediated by the endogenously secreted peptide IDA, which is directly recognized by the RLK HAE and the SERK co-receptors (Butenko et al., 2003; Cho et al., 2008; Meng et al., 2016; Santiago et al., 2016). Although the exact 
mechanism by which SOBIR1 is involved in organ abscission remains unknown, SOBIR1 appears to promote the internalization of HAE to mediate its negative role in regulating organ abscission (Leslie et al., 2010; Liljegren, 2012; Patharkar and Walker, 2018). Furthermore, it was recently shown that SOBIR1 prevents precocious fibre formation to control vascular development, in a manner that likely requires interaction with the RLK ERECTA (Milhinhos et al., 2019). However, the molecular mechanism behind the role of SOBIR1 in RLK-mediated developmental signalling remains to be elucidated.

Plasma membrane-associated RLKs and RLPs control complex formation of LRR-type cell surface receptors with their regulatory LRR-RLKs

As introduced above, LRR-type cell surface receptors form a complex with their regulatory LRR-RLKs. In addition, BAK1 and related SERKs also act as a co-receptor for some LRR-RLK receptors involved in defence and development. Emerging evidence reveals that these receptor complexes include additional plasma membraneassociated RLKs with either LRR or malectin-like domains, and LRR-RLPs. These regulatory proteins might not function as co-receptors but appear to control complex formation of LRR-type receptors with their regulatory LRR-RLKs (Fig. 1).

LRR-RLKs control complex formation of LRR-type receptors with their regulatory LRR$\underline{\mathrm{RLKs}}$

APEX is the paralogue of the SERKs and CIKs, and also contains five LRRs in its ectodomain (Smakowska-Luzan et al., 2018). Recently, it was shown that APEX functions as a scaffold to regulate the formation of receptor complexes (SmakowskaLuzan et al., 2018). For instance, APEX constitutively interacts with PEPR1/2 in a ligand-independent manner and controls PEPR1/2-mediated immune signalling in a dose-dependent manner (Smakowska-Luzan et al., 2018). In addition, APEX also plays a role in FLS2 and BRI1 signalling, and negatively regulates flg22-induced FLS2/BAK1 complex formation (Smakowska-Luzan et al., 2018) (Fig. 1). However, the molecular mechanism underlying the role of APEX in regulating complex formation remains elusive.

Arabidopsis BAK1-INTERACTING RECEPTOR-LIKE KINASE 2 (BIR2) and BIR3 also contain five LRRs and negatively regulate the formation of receptor complexes involved in defence and development (Halter et al., 2014a; Hohmann et al., 2018; Imkampe et al., 2017; Ma et al., 2017). In the resting state, BIR2 sequesters BAK1 from FLS2 (Halter et al., 2014b) (Fig. 1). Upon flg22 perception, BAK1 phosphorylates BIR2, resulting in its dissociation from BAK1 that subsequently associates with FLS2 (Halter et al., 2014b). Similar to BIR2, BIR3 also targets BAK1 to negatively regulate 
the flg22-induced formation of the FLS2/BAK1 complex, except that BIR3 also targets FLS2 (Imkampe et al., 2017) (Fig. 1). Additionally, BIR3 interacts with BRI1 and BAK1, and the association of BIR3 with BAK1 through their ectodomains and endodomains controls the formation of the BRI1/BAK1 complex (Großeholz et al., 2019; Halter et al., 2014b; Imkampe et al., 2017) (Fig. 1). Moreover, tomato BIR3 also interacts with BAK1 and negatively regulates FLS2 and BRI signalling (Huang et al., 2017). Furthermore, BAK1 employs distinct but overlapping regions of its ectodomain to interact with that of the BIRs and ligand-bound FLS2 and BRI1, while the latter two have a higher affinity for BAK1 than the BIRs (Hohmann et al., 2018; Ma et al., 2017). Currently, the molecular mechanism underlying the different roles of the BIRs in BAK1-dependent signalling pathways remains unknown, but it has been proposed that the endodomain of the BIRs, consisting of a juxta membrane domain and a kinase domain, might be responsible for their specific roles in different signalling pathways (Blaum et al., 2014; Hohmann et al., 2018; Ma et al., 2017).

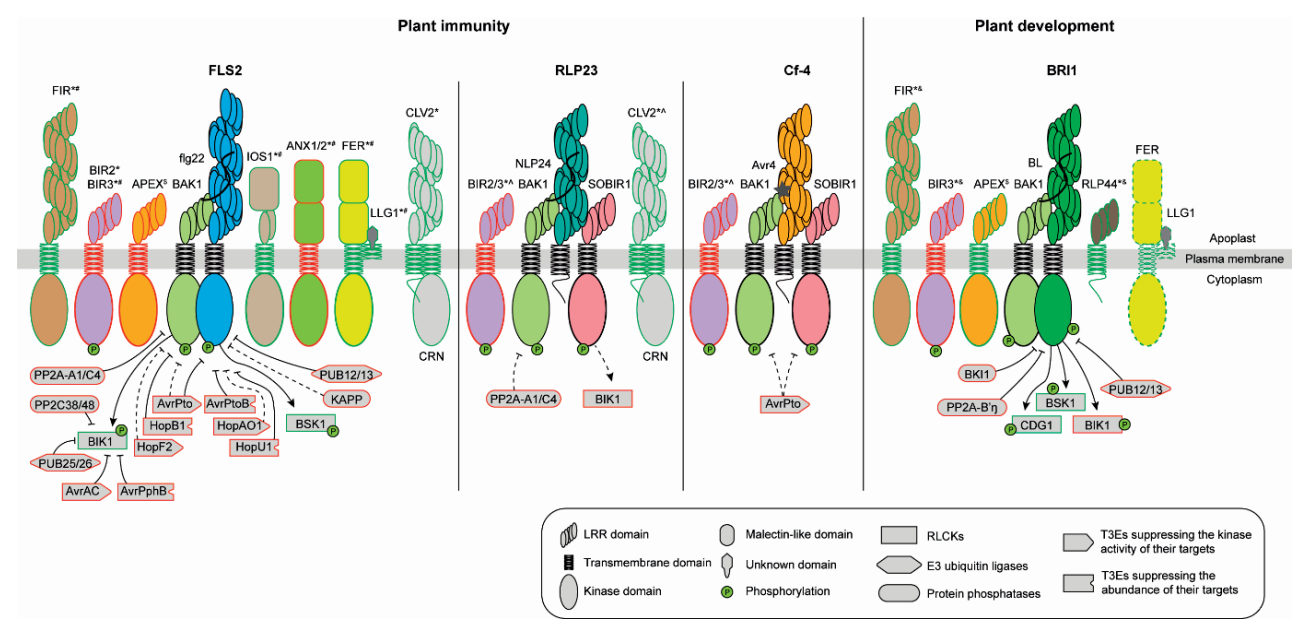

Figure 1. Overview of the regulators controlling the formation, activation and abundance of cell surface LRR-type receptor complexes involved in plant immunity and development. The RLK receptors FLS2 and BRI1 form a ligand-induced complex with their co-receptor BAK1 to mediate plant immunity and development, respectively. The RLP receptors RLP23 and Cf-4 constitutively interact with the regulatory RLK SOBIR1, and also recruit the regulatory RLK BAK1 upon ligand perception, leading to activation of defence responses. Plasma membrane-associated proteins interacting with FLS2, BRI1, BAK1 and SOBIR1 are marked by \#, \&, * and ${ }^{\wedge}$. In addition, proteins marked by ${ }^{\$}$ perform their regulatory roles probably through controlling complex formation of receptors with the co-receptor BAK1. Note that the E3 ubiquitin ligases PUB12/13 ubiquitinate FLS2 in a manner depending on BAK1, and that the Pst DC3000 effector AvrPtoB directly suppresses kinase activity of BAK1. Positive and negative regulators of these receptor complexes are indicated by green and red boxes, respectively. Possible regulatory roles and proposed functions are indicated by dashed boxes and dashed lines, respectively (see text for details). 
Besides negatively regulating signalling mediated by RLKs, BIR2 and BIR3 also negatively regulate immune responses mediated by RLPs (Fig. 1). For example, BIR2 and BIR3 were found to negatively regulate RLP23 signalling in Arabidopsis (Chapter 2 ), which is consistent with a recent report describing that RLP23 signalling is enhanced in bir2 loss-of-function mutants (Wan et al., 2018). In addition, BIR2 and BIR3 also suppress Cf-4-mediated signalling (Chapter 2). These observations are reminiscent of the negative role of BIR2 and BIR3 in FLS2 and BRI1 signalling (Halter et al., 2014a; Imkampe et al., 2017). Since BAK1 forms a ligand-induced complex with the RLKs FLS2 and BRI1 and with the RLPs RLP23 and Cf-4 (Albert et al., 2015; Chinchilla et al., 2007; He et al., 2007; Liebrand et al., 2013), BIR2 and BIR3 probably also target BAK1 to inhibit ligand-induced association of BAK1 with RLPs (Fig. 1). Notably, BIR2 and BIR3 also target SOBIR1 to negatively regulate RLP-mediated immune signalling (Fig. 1), since these two BIR members interact with SOBIR1, and even appear to inhibit the formation of the constitutive Cf-4/SOBIR1 complex (Chapter 2). So far, the molecular mechanisms behind the interaction of SOBIR1 with BIR2 and BIR3 and the inhibition of Cf-4/SOBIR1 complex formation by these two BIR members remain unknown. Further research is needed to address which domains of the BIRs and of SOBIR1 are required for their interaction. In addition, further research on whether the BIRs employ a similar mechanism to interact with SOBIR1 and BAK1 would shed light on our understanding of the role of the BIRs in controlling the formation and activation of the Cf-4/SOBIR1/BAK1 complex.

BIR1 and BIR4 are paralogues of BIR2 and BIR3 (Halter et al., 2014b). Similar to BIR2 and BIR3, BIR1 and BIR4 also have five LRRs and interact with SOBIR1 and BAK1 (Halter et al., 2014b; Hohmann et al., 2018; Ma et al., 2017) (Chapter 2). However, it is worth noting that BIR1 and BIR4 function differently from BIR2 and BIR3 in regulating defence. For instance, unlike the other BIRs, BIR1 is a kinase-active RLK (Blaum et al., 2014; Gao et al., 2009; Halter et al., 2014a; Imkampe et al., 2017). In addition, the knockout mutant bir1-1 causes auto-immunity, which is proposed to depend on the activation of unknown NB-LRRs that might guard the integrity of BIR1 (Gao et al., 2009). Moreover, this auto-immunity also depends on SOBIR1 and BAK1, which form a complex in the absence of BIR1 (Gao et al., 2009; Liu et al., 2016). Formation of the SOBIR1/BAK1 complex subsequently activates downstream immune responses, thereby contributing to the auto-immunity of bir1-1 (Gao et al., 2009; Liu et al., 2016). Notably, BIR1 is involved in antiviral infection in a manner that is likely independent of its negative role in triggering auto-immunity (Guzmán-Benito et al., 2019). Furthermore, BIR1 does not affect immune responses mediated by FLS2 and RLPs (Gao et al., 2009; Liu et al., 2016) (Chapter 2). Additionally, unlike the other BIRs that negatively regulate immune responses, BIR4 functions as a positive regulator of immune responses 
mediated by RLPs (Chapter 2). The molecular mechanism behind the different roles of the BIRs remains largely unknown, but the different roles of the BIRs might also be determined by the interactions with SOBIR1 and BAK1 through their endodomains.

RLKs containing similar amounts of LRRs in their ectodomain as ligand-binding receptors can also control the formation of LRR-RLK receptor complexes, which is exemplified by the RLK FLS2-INTERACTING RECEPTOR (FIR) (Smakowska-Luzan et al., 2018). FIR interacts with both FLS2 and BAK1, and positively regulates flg22induced FLS2/BAK1 complex formation (Smakowska-Luzan et al., 2018) (Fig. 1). In addition, FIR also interacts with BRI1 and is required for BRI1 signalling (SmakowskaLuzan et al., 2018) (Fig. 1). So far, it remains unknown whether FIR regulates the association of BAK1 with BRI1, as well as with other RLKs and RLPs involved in defence and development.

LRR-RLPs control complex formation of LRR-type receptors with their regulatory LRR$\underline{\mathrm{RLKS}}$

Recently, it has been reported that RLP44 participates in the regulation of development through the activation of BRI1 and PSKR1 signalling (Holzwart et al., 2018; Wolf et al., 2014). RLP44 constitutively interacts with BRI1, PSKR1 and BAK1, and functions as a scaffold to stabilize the BRI1/BAK1 (Fig. 1) and PSKR1/BAK1 complexes (Holzwart et al., 2018; Wolf et al., 2014). Furthermore, the RLP TMM participates in the regulation of stomatal development, a process that is mediated by the secreted peptides EPF1/2 (Hara et al., 2007; Hunt and Gray, 2009). TMM forms a constitutive complex with the ERfs to directly recognize EPF1/2 (Lee et al., 2012; Lin et al., 2017). Moreover, TMM also constitutively interacts with the SERKs and probably stabilizes the ligand-induced interaction between the ERfs and the SERKs (Meng et al., 2015). TMM also interacts with SOBIR1 (Liebrand et al., 2013), but the biological function of the TMM/SOBIR1 complex remains unknown.

The RLP CLV2 might also function as a regulatory protein to control receptor complex formation. CLV2 forms a constitutive complex with the pseudokinase CORYNE (CRN) to participate in various CLE signalling pathways (Fiers et al., 2005; Jeong et al., 1999; Kayes and Clark, 1998; Miwa et al., 2008). For instance, the CLV2/CRN complex participates in CLV3 signalling through the employment of CRN to interact with CLV1 and the CIK regulatory RLKs (Bleckmann et al., 2010; Durbak and Tax, 2011; Hu et al., 2018; Zhu et al., 2010b). In addition, a recent report revealed that CLV2 itself also constitutively interacts with CIK2 (Ren et al., 2019). However, it remains unknown whether the CLV2/CRN complex regulates the interaction between CLV1 and the CIKs. In addition, the CLV2/CRN complex is involved in BAM3-mediated CLE45 signalling, 
probably through regulating the interaction between BAM3 and yet unknown regulatory RLKs, which might be the CIKs as they are also required for CLE45 signalling (Anne et al., 2018; Hazak et al., 2017; Hu et al., 2018).

Besides functioning in development, the CLV2/CRN complex also participates in defence responses mediated by RLPs and FLS2 (Chapter 5, and Fig. 1). Similar to RLPs involved in defence and TMM, CLV2 also constitutively interacts with SOBIR1 (Gust and Felix, 2014; Liebrand et al., 2013; Liebrand et al., 2014; van der Burgh and Joosten, 2019; Wan et al., 2019) (Chapter 5). A previous study revealed that the Gxx G motif in the transmembrane domain of SOBIR1 is required for its interaction with CLV2 and Cf-4, of which the latter also carries such a GxxxG motif (Bi et al., 2016). However, this motif is not present in the transmembrane domain of CLV2 and TMM (Gust and Felix, 2014), suggesting that this motif is dispensable for RLPs to interact with SOBIR1, or that these RLPs interact with SOBIR1 in different ways. Since the ectodomain of SOBIR1 is required for Cf-4 functionality (Bi et al., 2016), the ectodomain of SOBIR1 and the C-terminal part of the ectodomain of Cf-4 probably interact to allow ligand perception or signal initiation. Considering that the C-terminal part of the ectodomain of Cf-4 is different from that of CLV2 and TMM (Gust and Felix, 2014), these RLPs might interact with SOBIR1 in different ways. Furthermore, CLV2 also constitutively interacts with BAK1 (Chapter 5), an observation that is divergent from the ligandinduced association of BAK1 with cell surface receptors (Gust and Felix, 2014; Liebrand et al., 2014; Ma et al., 2016; van der Burgh and Joosten, 2019; Wan et al., 2019). However, the observed constitutive interaction between BAK1 and CLV2 is reminiscent of the constitutive association of BAK1 with RLP44 and TMM (Meng et al., 2015; Wolf et al., 2014), indicating that BAK1 might employ a different mechanism to interact with RLPs that do not function as ligand-binding receptors. This speculation is supported by the fact that the amino acid sequence of CLV2, TMM and RLP44 is divergent in the C-terminal part of the ectodomain, which is anticipated to be important for RLPs to interact with BAK1 (Catanzariti et al., 2017; Gust and Felix, 2014). The molecular mechanism behind the role of the CLV2/CRN complex in regulating defence responses remains unknown. However, since the CLV2/CRN complex controls the accumulation of its interactors (Hazak et al., 2017; Jeong et al., 1999), this complex may regulate the accumulation of the SOBIR1 and BAK1 proteins, thereby indirectly regulating plant defence responses. Furthermore, since SOBIR1 is required for the accumulation of Cf-4 (Liebrand et al., 2013), SOBIR1 might also facilitate the accumulation of CLV2, thereby participating in CLV2-dependent CLV3 signalling to regulate rosette leaf numbers (Chapter 5). 
Malectin-like domain-containing RLKs control complex formation of LRR-type receptors and their regulatory LRR-RLKS

IMPAIRED OOMYCETE SUSCEPTIBILITY 1 (IOS1) contains two LRRs and a malectin-like domain in its ectodomain (Hok et al., 2011). IOS1 is required for immune responses mediated by various cell surface receptors, including FLS2 and EFR (Yeh et al., 2016). In addition, IOS1 constitutively interacts with FLS2, EFR and BAK1, and positively regulates flg22-induced FLS2/BAK1 complex formation (Yeh et al., 2016) (Fig. 1).

It is worth noting that ligand-binding RLKs containing malectin-like domains can also function as scaffolds to control complex formation of LRR-RLK receptors with their regulatory LRR-RLKs (Franck et al., 2018; Li et al., 2016a). For instance, ANXUR1 (ANX1) and ANX2, which both contain two malectin-like domains in their ectodomain, function redundantly to prevent pollen tube rupture during fertilization upon recognition of the endogenously secreted peptides RAPID ALKALINIZATION FACTOR 1 (RALF4) and RALF19 (Boisson-Dernier et al., 2009; Ge et al., 2017; Miyazaki et al., 2009). In addition, ANX1 and ANX2 function redundantly to negatively regulate plant immunity (Mang et al., 2017). Moreover, ANX1 constitutively interacts with FLS2 and forms a ligand-stimulated complex with BAK1 (Mang et al., 2017) (Fig. 1). However, the association of ANX1 with BAK1 suppresses flg22-induced FLS2/BAK1 complex formation (Mang et al., 2017).

The RLK FERONIA (FER) is the closest paralogue of ANX1 and ANX2 (Li et al., 2016a). FER contains two malectin-like domains and recognizes yet unknown RALF peptides to regulate pollen tube reception during fertilization (Escobar-Restrepo et al., 2007; Huck et al., 2003; Rotman et al., 2003). In addition, FER directly recognizes RALF1 and RALF23 to inhibit seedling growth (Haruta et al., 2014; Stegmann et al., 2017). Similar to ANX1, FER also constitutively interacts with FLS2 and associates with BAK1 in a ligand-stimulated manner (Stegmann et al., 2017) (Fig. 1). However, unlike ANX1, FER positively regulates flg22-induced FLS2/BAK1 complex formation (Stegmann et al., 2017).

Recently, it was shown that the glycosylphosphatidylinositol-anchored protein LORELEI-LIKE GPI-ANCHORED PROTEIN 1 (LLG1) heterodimerizes with FER in a RALF23-dependent manner (Xiao et al., 2019). Similar to FER, LLG1 is also required for flg22-induced FLS2/BAK1 complex formation through its interaction with FLS2 and BAK1 (Shen et al., 2017; Xiao et al., 2019) (Fig. 1). In addition, LLG1 is also required for the phosphorylation of the RLCK BOTRYTIS-INDUCED KINASE1 (BIK1) (Shen et al., 2017), which is a direct phosphorylation substrate of the FLS2/BAK1 complex (Lin 
et al., 2014; Lu et al., 2010; Zhang et al., 2010). Interestingly, activation of RALF23/LLG1/FER signalling inhibits flg22-induced FLS2/BAK1 complex formation, leading to the suppression of FLS2 signalling (Stegmann et al., 2017; Xiao et al., 2019). Notably, it has also been reported that RALF23 suppresses BRI1 signalling (Srivastava et al., 2009), suggesting that the FER/LLG1 complex also regulates BRI1/BAK1 complex formation (Fig. 1). So far, the molecular mechanism behind the negative role of RALF23 on the formation of these LRR-type receptor complexes through the FER/LLG1 complex remains elusive. In addition, it also remains unknown whether the FER/LLG1 complex regulates the formation of additional LRR-type receptor complexes.

\section{RLCKs as phosphorylation substrates of LRR-type cell surface receptor complexes}

RLCKs as phosphorylation substrates of LRR-RLK receptor complexes involved in defence

Emerging evidence reveals that RLCKs function as phosphorylation substrates of LRR-RLK receptor complexes involved in defence (Cui et al., 2018; Liang and Zhou, 2018). The Arabidopsis RLCK family contains 149 members that can be subdivided into 17 subfamilies based on the amino acid sequence of their kinase domain (Shiu et al., 2004). In addition, the RLCK VII subfamily consists of 46 members that can be further subdivided into nine clades based on the full-length amino acid sequence of these proteins (Rao et al., 2018).

Recently, it was reported that members of the RLCK VII-5, -7 and -8 clades are redundantly required for FLS2 and EFR signalling (Rao et al., 2018). Among these RLCKs, the RLCK VII-8 clade member BIK1 for example is a direct phosphorylation substrate of the FLS2/BAK1 complex (Lin et al., 2014; Lu et al., 2010; Zhang et al., 2010) (Fig. 1). In the resting state, BIK1 interacts with both FLS2 and BAK1 (Lu et al., 2010; Zhang et al., 2010). Upon flg22 perception, activated BAK1 phosphorylates BIK1, which in its turn trans-phosphorylates and subsequently dissociates from the FLS2/BAK1 complex (Lin et al., 2014; Lu et al., 2010; Zhang et al., 2010). Additionally, members of the RLCK VII-6 clade function redundantly to negatively regulate FLS2 signalling (Rao et al., 2018). Among these RLCK VII-6 members, AVRPPHB SUSCEPTIBLE 1-LIKE 13 (PBL13) dynamically interacts with the plasma membraneassociated NADPH oxidase RESPIRATORY BURST OXIDASE HOMOLOG D (RBOHD), which is the key enzyme mediating ROS production in FLS2 signalling (Kadota et al., 2014; Li et al., 2014b; Lin et al., 2015). Kinase activity of PBL13 is required for its negative role in FLS2 signalling (Lin et al., 2015), suggesting that PBL13 might get phosphorylated and dissociate from RBOHD upon activation of the FLS2/BAK1 complex. 
Members from other RLCK families are also required for FLS2 signalling. For instance, the RLCK XII subfamily member BRASSINOSTEROID-SIGNALING KINASE 1 (BSK1) constitutively interacts with FLS2 (Shi et al., 2013) (Fig. 1). Upon flg22 perception, BSK1 dissociates from FLS2 (Shi et al., 2013), a process that is likely driven by phosphorylation of BSK1. In addition, perception of flg22 triggers the phosphorylation of the tomato RLCK VIII subfamily member PTO-INTERACTING PROTEIN1b (PTI1b), of which its kinase activity is required for FLS2 signalling (Giska and Martin, 2019; Schwizer et al., 2017). However, it remains unknown whether phosphorylation of Pti1b is directly mediated by FLS2 or BAK1.

RLCKs as phosphorylation substrates of LRR-RLK receptors involved in development Besides being a positive regulator of FLS2 signalling, BIK1 and BSK1 also play a role in BRI1 signalling. BIK1 constitutively interacts with BRI1, while activated BRI1 phosphorylates BIK1 leading to its dissociation from BIR1 (Lin et al., 2013a) (Fig. 1). The dynamic association of the BRI1/BIK1 complex is reminiscent of the FLS2/BIK1 complex (Lin et al., 2013a; Lu et al., 2010; Zhang et al., 2010). However, unlike its positive role in FLS2 signalling, BIK1 negatively regulates BRI1 signalling (Lin et al., 2013a; Lu et al., 2010; Zhang et al., 2010). In addition, BAK1 is required for phosphorylation of BIK1 and for the dissociation of the FLS2/BIK1 complex, whereas BAK1 is not required for BRI1-mediated direct phosphorylation of BIK1 and for the dissociation of the BRI1/BIK1 complex (Lin et al., 2014; Lin et al., 2013a; Lu et al., 2010; Zhang et al., 2010). Although it is not fully understood, the differential phosphorylation of BIK1 by BAK1 and BRI1 might determine the different roles of BIK1 in FLS2 and BRI1 signalling (Lin et al., 2013a). Since BRI1 is an RD kinase with strong kinase activity, while FLS2 is a non-RD kinase with weak kinase activity (Schwessinger et al., 2011), it appears that the BAK1-independent phosphorylation of BIK1 triggered by an $\mathrm{RD}$ kinase receptor might determine a negative role of BIK1. Unlike BIK1 playing different roles in BRI1 and FLS2 signalling, BSK1 is a positive regulator for both BRI1 and FLS2 signalling (Tang et al., 2008) (Fig. 1). Similar to the dynamic association of the FLS2/BSK1 complex, BSK1 also dissociates from BRI1 upon treatment with BL (Tang et al., 2008). In addition, BRI1 directly phosphorylates BSK1 (Tang et al., 2008). Furthermore, the RLCK VII subfamily member CONSTITUTIVE DIFFERENTIAL GROWTH 1 (CDG1) is required for BRI1 signalling, while BRI1 directly interacts with, and phosphorylates CDG1 (Kim et al., 2011) (Fig. 1).

RLCK VII members are also required for signalling triggered by other LRR-RLK receptors. The RLCK VII-7 clade member PBL30, which is also known as CAST AWAY (CST), functions as an inhibitor of organ abscission likely through cooperating with SOBIR1 in regulating the internalization of HAE (Burr et al., 2011; Liljegren, 2012). 
CST interacts with HAE and SOBIR1 (Burr et al., 2011), but it remains unknown whether CST is phosphorylated by these two kinases. Furthermore, members of the RLCK VII-6 and -8 clades are redundantly required for PSKR1-mediated regulation of root elongation (Rao et al., 2018). As the positive regulatory role of the RLCK VII-8 clade member BIK1 in FLS2 signalling appears to depend on BAK1-mediated phosphorylation, it is likely that BIK1 is also phosphorylated by BAK1 upon activation of PSKR1 signalling.

\section{RLCKs involved in immune signalling pathways mediated by LRR-RLP receptors}

RLCKs acting as a direct phosphorylation substrate of the RLP/SOBIR1/BAK1 complex have not been reported yet. Recently, it was reported that BIK1 negatively regulates RLP23 signalling (Wan et al., 2018). As discussed above, BIK1 also negatively regulates developmental signalling mediated by the RD kinase BRI1, and this negative role of BIK1 appears to depend on BRI1-mediated direct phosphorylation of BIK1 in a BAK1-independent manner (Lin et al., 2013a). Hence, it is likely that the negative regulatory role of BIK1 in RLP23 signalling might be caused by phosphorylation of BIK1 via an unknown RD kinase. In this scenario, a potential candidate can be SOBIR1, which is an RD kinase and constitutively interacts with RLP23 to form a bi-molecular RD RLK (Albert et al., 2015; Gust and Felix, 2014; Liebrand et al., 2014; van der Burgh et al., 2019) (Fig. 1). Further characterization of the involvement of the various identified BIK1 phospho-sites (Lin et al., 2014; Xu et al., 2013) for its phosphorylation mediated by SOBIR1, but not by BAK1, will help to understand whether indeed the negative role of $\mathrm{BIK} 1$ is specified by its phosphorylation by an RD kinase, like SOBIR1 and BRI1, in a manner independent of BAK1. Furthermore, the RLCK VII-6 clade member Avr9/Cf-9 INDUCED KINASE 1 (ACIK1) is required for the HR triggered by Cf-9/Avr9 and Cf-4/Avr4 in N. benthamiana (Rowland et al., 2005). ACIK1 is the orthologue of Arabidopsis PBL13, which negatively regulates FLS2 signalling and targets RBOHD to prevent flg22-triggered ROS production (Lin et al., 2015). In addition, $\mathrm{NbRBOHB}$, the orthologue of Arabidopsis RBOHD, negatively regulates the development of the Cf-9/Avr9-triggered HR (Zhu et al., 2010a). Collectively, these data suggest that ACIK1 exerts its positive role in regulating the Cf-9/Avr9-triggered HR probably through targeting $\mathrm{NbRBOHB}$.

\section{Intracellular protein phosphatases regulate the activation of LRR-type cell surface receptor complexes}

So far, some protein phosphatases have been shown to participate in various cellular processes through dephosphorylating different targets, which include ligand-binding receptors, regulatory LRR-RLKs and downstream RLCKs (Couto and Zipfel, 2016; Mithoe and Menke, 2018; Uhrig et al., 2013). Here, an overview of phosphatases 
targeting receptor complexes involved in defence and development is provided.

Protein phosphatases dephosphorylate LRR-RLK receptor complexes involved in defence

Several protein phosphatases have been shown to target FLS2, BAK1 and BIK1 to negatively regulate FLS2 signalling. For instance, the protein phosphatase type $2 \mathrm{C}$ (PP2C) subunit KINASE ASSOCIATED PROTEIN PHOSPHATASE (KAPP) targets FLS2 to negatively regulate flg22-triggered immune responses (Gómez-Gómez et al., 2001) (Fig. 1). In addition, the serine/threonine protein phosphatase type 2A (PP2A) subunits A1, B' $\eta, B$ ' $\zeta$ and $C 4$ constitutively interact with BAK1 and suppress its kinase activity (Segonzac et al., 2014) (Fig. 1). Moreover, the PP2C subunits PP2C38 and PP2C48 function redundantly to negative regulate FLS2 signalling through targeting the FLS2/BIK1 complex (Couto et al., 2016). PP2C38 dephosphorylates BIK1, while activated BIK1 phosphorylates this protein phosphatase subunit, leading to its dissociation from both FLS2 and BIK1 (Couto et al., 2016) (Fig. 1).

Kinase activity of some other LRR-RLK receptors involved in defence is also controlled by protein phosphatases. For instance, XA21-mediated defence is negatively regulated by the ATPase XB24 (Chen et al., 2010). XB24 interacts with XA21 and keeps XA21 in an inactive state by promoting auto-phosphorylation of XA21 on specific residues, while ligand binding triggers the dissociation of the XA21/XB24 complex and the activation of XA21 (Chen et al., 2010). Activated XA21 associates with the PP2C subunit XA21 BINDING PROTEIN 15 (XB15), which targets and dephosphorylates XA21 to negatively regulate plant immunity (Park et al., 2008). Functional orthologues of XB24 and XB15 also regulate plant immunity in Arabidopsis (Holton et al., 2015). Similar to the interaction between XB24 and XA21, the Arabidopsis XB24 orthologue dissociates from EFR upon elf18 perception (Holton et al., 2015). However, unlike XB24, Arabidopsis XB24 appears to be a positive regulator of EFR signalling, which might be caused by a mutation in its ATPase motif (Holton et al., 2015). Furthermore, the Arabidopsis XB15 orthologues POLTERGEIST-LIKE 4 (PLL4) and PLL5 also negatively regulate EFR signalling, despite that PLL4 and PLL5 dissociate from EFR upon elf18 perception (Holton et al., 2015).

Protein phosphatases dephosphorylate LRR-RLK receptors involved in development Besides targeting FLS2, KAPP also targets and dephosphorylates CLV1 to negatively regulate CLV3 signalling (Stone et al., 1998; Williams et al., 1997). Furthermore, kinase activity of BRI1 is regulated by the PP2C subunit BRI1 KINASE INHIBITOR 1 (BKI1), which negatively regulates BRI1 signalling (Wang and Chory, 2006) (Fig. 1). In the resting state, BKI1 constitutively associates with BRI1 to prevent its activation (Wang 
and Chory, 2006). Upon BL perception, activated BRI1 phosphorylates BKI1, resulting in its dissociation from BRI1 (Jaillais et al., 2011; Wang and Chory, 2006). Furthermore, a group of PP2A subunits negatively regulates BRI1 signalling, and the PP2A subunit B'n predominantly targets and dephosphorylates activated BRI1 (Wang et al., 2016b) (Fig. 1).

Protein phosphatases dephosphorylate LRR-RLP receptor complexes involved in defence

Currently, protein phosphatases directly targeting the RLP/SOBIR1/BAK1 complex involved in defence remain unknown. However, BAK1, SOBIR1 and possibly the RLP itself might be targets of yet unknown protein phosphatases.

Recently, it was reported that RLP23 signalling is negatively regulated by the PP2A subunits A1 and C4 (Wan et al., 2018). Since PP2A-A1 and PP2A-C4 target and dephosphorylate BAK1 to negatively regulate FLS2 signalling (Segonzac et al., 2014), these two protein phosphatases probably also target BAK1 to negatively regulate RLP23 signalling (Fig. 1).

The kinase activity of SOBIR1 might also be regulated by yet unknown phosphatases (van der Burgh et al., 2019) (Chapter 3). SOBIR1 is a positive regulator of plant immunity and overexpression of SOBIR1 triggers constitutive immunity in Arabidopsis (Gao et al., 2009). Consistent with this finding, transient overexpression of Arabidopsis SOBIR1 in $N$. benthamiana and $N$. tabacum also triggers constitutive immunity, which is associated with the development of an HR (Chapter 3). The Arabidopsis SOBIR1triggered constitutive immunity is proposed to be caused by auto-phosphorylation of SOBIR1, which subsequently trans-phosphorylates BAK1 that promotes phosphorylation of SOBIR1 in turn (van der Burgh et al., 2019). In addition, Arabidopsis SOBIR1-triggered constitutive immunity also involves the activation of downstream MAPKs (Chapter 3). Similar phosphorylation events might also occur upon activation of RLP/SOBIR1/BAK1-mediated signalling (van der Burgh et al., 2019). However, transient overexpression of SOBIR1 orthologues of tomato and $N$. benthamiana does not trigger an HR (Chapter 3). Since the essential kinase motifs are highly conserved among the various SOBIR1 orthologues (van der Burgh, 2018), the differential ability of the various SOBIR1 orthologues in triggering constitutive immunity might be caused by non-conserved regions in their kinase domains. Alternatively, yet unknown protein phosphatases in $N$. benthamiana might have a lower affinity for the kinase domain of Arabidopsis SOBIR1, which may result in the failure to keep overexpressed Arabidopsis SOBIR1 in check by dephosphorylation. Compromised dephosphorylation might lead to excessive phosphorylation of SOBIR1, which results in SOBIR1-triggered 
constitutive immunity (van der Burgh et al., 2019) (Chapter 3).

Besides SOBIR1 and BAK1, the LRR-RLP receptors themselves might also be targets of protein phosphatases. Research on cell surface chimeric receptor proteins revealed that the ectodomain of a chimera retains the ligand perception ability, while the transmembrane and cytoplasmic domains maintain the signalling output intensity (Albert et al., 2010; Brutus et al., 2010; He et al., 2000; Holton et al., 2015; Kishimoto et al., 2010; Kouzai et al., 2013; Schwessinger et al., 2015; Thomas et al., 2017). Based on this observation, an attempt was made to engineer the immune system of $N$. tabacum plants for providing a strong defence response against bacterial infection, by exploiting the EFR-mediated recognition of the MAMP elf18 and the ability of Cf- 9 to trigger a strong HR. To reach this goal, the chimeric receptor protein EFR-Cf- 9 was generated by fusing the ectodomain of EFR to the transmembrane and cytoplasmic domains of Cf-9, which are in fact identical to those of Cf-4 (Thomas et al., 1997). Transient and stable transgenic assays in $N$. tabacum revealed that the EFR-Cf- 9 chimera recognizes the MAMP elf18 to trigger a strong HR (Chapter 4), which is similar to the HR triggered by Cf-9/Avr9 and Cf-4/Avr4. In addition, transgenic N. tabacum plants expressing the chimera EFR-Cf-9 mediate elf18-induced defence that arrests the growth of biotrophic bacterial pathogens (Chapter 4). Notably, these transgenic plants even trigger an HR upon inoculation with a high dose of a bacterial pathogen (Chapter 4). Furthermore, the elf18/EFR-Cf-9-triggered HR was shown to depend on SOBIR1 and BAK1 (Chapter 4). As SOBIR1 is not required for EFR signalling, it was concluded that fusing the transmembrane and cytoplasmic domains of Cf-9 to the ectodomain of EFR enables the chimeric protein EFR-Cf-9 to interact with SOBIR1 and trigger an HR upon treatment with elf18 (Chapter 4). Since the transmembrane domain of Cf-9 is overall similar to that of other RLPs, whereas the cytoplasmic tail of RLPs is variable (Gust and Felix, 2014), it is likely that the cytoplasmic tail of Cf-9 is responsible for the elf18/EFR-Cf-9-triggered HR. Indeed, a recent study revealed that the cytoplasmic tail of Cf- 9 is important for its functionality, as substitution of the putative phospho-site threonine $(\mathrm{T})$ at position 835 by aspartate, which mimics phosphorylation, attenuates the Cf-9/Avr9-triggered HR (Chakrabarti et al., 2016). This observation suggests that the cytoplasmic C-terminal tail of Cf-9 might be phosphorylated at T835 to keep Cf-9 in an inactive resting state, while yet unknown protein phosphatases prevent phosphorylation of T835 to sustain Cf-9 signalling upon recognition of Avr9. Furthermore, it has been recently reported that RLP44 is phosphorylated on the serine (S) residue at position 268 in its cytoplasmic tail (Gómez et al., 2019). Notably, phosphorylation of RLP44 at S268 is responsible for its interaction with BRI1 and its function in BR signalling activation, whereas the phosphorylation status at this phospho-site does not affect RLP44 interaction with PSKR1 and its function in PSK 
signalling activation (Gómez et al., 2019; Wolf et al., 2014). Although the specific protein phosphatases controlling the phosphorylation status of Cf-9 and RLP44 remain unknown, these findings support the notion that the cytoplasmic tail of RLPs is required for their functionality and plays a role in determining the signalling output in a phosphorylation-dependent manner.

\section{Intracellular E3 ubiquitin ligases regulate the abundance of LRR-type cell surface receptor complexes}

E3 ubiquitin ligases are a class of enzymes that ubiquitinate proteins involved in various cellular processes, resulting in degradation of these proteins (Chen and Hellmann, 2013). Currently, E3 ubiquitin ligases have been shown to negatively regulate the abundance of LRR-RLK receptor complexes involved in defence and development (Couto and Zipfel, 2016; Li et al., 2014a; Mithoe and Menke, 2018). E3 ubiquitin ligases that either positively or negatively regulate immune responses mediated by LRR-RLPs have also been reported (Gilroy et al., 2011; GonzálezLamothe et al., 2006; He et al., 2015; Orosa et al., 2017; Rowland et al., 2005; van den Burg et al., 2008; Yang et al., 2006), but E3 ubiquitin ligases directly targeting LRR$\mathrm{RLP}$ receptor complexes remain currently unknown.

E3 ubiquitin ligases regulate the abundance of LRR-RLK receptor complexes involved in defence

Several plant U-box (PUB) E3 ubiquitin ligases have been shown to target the FLS2/BAK1/BIK1 complex to negatively regulate FLS2 signalling. For instance, PUB12 and PUB13 (further referred to as PUB12/13) constitutively interact with the kinase domain of BAK1 (Lu et al., 2011). Upon flg22 perception, activated BAK1 phosphorylates PUB12/13, which subsequently associate with FLS2 and ubiquitinate this receptor for degradation (Lu et al., 2011) (Fig. 1). The abundance of the BIK1 protein is negatively regulated by PUB25 and PUB26 (Fig. 1), as well as by their closest paralogues PUB22 and PUB23 (Trujillo et al., 2008; Wang et al., 2018a). PUB25 and PUB26 predominantly interact with unphosphorylated BIK1, and ubiquitinate nonactivated BIK1 to trigger its degradation (Wang et al., 2018a).

In rice, the E3 ubiquitin ligase $\mathrm{XB} 3$ is a positive regulator of $\mathrm{XA} 21$ signalling (Wang et al., 2006). XA21 interacts with and phosphorylates $X B 3$, which positively regulates the accumulation of XA21 protein through an unknown mechanism (Wang et al., 2006).

E3 ubiquitin ligases regulate the abundance of LRR-RLK receptors involved in development

Besides ubiquitinating FLS2 for degradation, PUB12/13 also negatively regulate the 
abundance of BRI1 protein (Fig. 1), despite the fact that the molecular mechanisms underlying PUB12/13-mediated degradation of FLS2 and BRI1 are different (Lu et al., 2011; Zhou et al., 2018). For instance, BAK1 is required for phosphorylation of PUB12/13 and their subsequent association with FLS2, whereas BRI1 forms a ligandstimulated complex with PUB12/13 and directly phosphorylates these two E3 ubiquitin ligases (Lu et al., 2011; Zhou et al., 2018). BRI1-mediated phosphorylation of PUB12/13 results in their enhanced activity, which promotes the ubiquitination and degradation of BRI1 (Zhou et al., 2018).

E3 ubiquitin ligases regulate the abundance of signalling components required for immune responses mediated by LRR-RLP receptors

E3 ubiquitin ligases that directly target LRR-RLP receptor complexes involved in defence remain unknown. However, four E3 ubiquitin ligases Avr9/Cf-9 RAPIDLY ELICITED 74 (ACRE74), ACRE189 and ACRE276 have been reported to play a role in Cf-4 and Cf-9 signalling with yet unknown mechanisms (Gilroy et al., 2011; González-Lamothe et al., 2006; Rowland et al., 2005; van den Burg et al., 2008; Yang et al., 2006). Furthermore, it has been shown that the E3 ubiquitin ligase POZ/BTB CONTAINING G-PROTEIN 1 (POB1) negatively regulates Cf-4 and Cf-9 signalling through ubiquitinating ACRE276 for degradation (He et al., 2015; Orosa et al., 2017).

\section{Bacterial effectors target LRR-type cell surface receptor complexes to suppress defence}

To successfully colonize host cells, pathogens secrete extracellular and/or intracellular effectors to suppress defence triggered by cell surface receptors (van der Burgh and Joosten, 2019). For instance, C. fulvum secretes the lysin-motif-containing extracellular effector EXTRACELLULAR PROTEIN 6 (Ecp6) to bind chitin fragments that are released from its cell walls by plant chitinases, resulting in inhibition of chitintriggered immunity (Bolton et al., 2008; de Jonge et al., 2010). In addition, the bacterial pathogen Pseudomonas syringae pv. tomato (Pst) DC3000 employs its type III secretion system (T3SS) to inject at least 28 different T3SS effectors (T3Es) into its host cells (Cunnac et al., 2009). In addition, some of these T3Es have been shown to suppress FLS2 signalling in different ways (Büttner, 2016; Lee et al., 2013; Xin and He, 2013). Here, an overview is provided of bacterial T3Es regulating kinase activity and abundance of receptor complexes involved in defence.

T3Es suppress the activation of cell surface receptor complexes

The Pst DC3000 T3E AvrPto is a well-known bacterial effector that suppresses defence responses triggered by various cell surface receptors (Büttner, 2016; He et al., 2006; Shan et al., 2008). AvrPto interacts with the kinase domains of FLS2 and EFR, and 
functions as a kinase inhibitor to suppress auto-phosphorylation of these two RLK receptors (Xiang et al., 2008) (Fig. 1). In addition, the association of AvrPto with FLS2 inhibits flg22-induced phosphorylation of BIK1 and subsequent dissociation of the FLS2/BIK1 complex, thereby suppressing FLS2 signalling (Xiang et al., 2011; Zhang et al., 2010). Moreover, AvrPto also interacts with BAK1 and inhibits the flg22-induced formation of the FLS2/BAK1 complex, although contradicting reports on this aspect have been published (Shan et al., 2008; Xiang et al., 2011; Zhou et al., 2014). Still, these findings suggest that AvrPto functions as a kinase inhibitor and probably also as a regulatory protein to control the formation of the FLS2/BAK1 complex to suppress FLS2 signalling. Furthermore, AvrPto even inhibits BL-induced BRI1/BAK1 complex formation, leading to compromised BRI1 signalling (Shan et al., 2008). AvrPto does not interact with BRI1 and appears not to suppress the BL-induced phosphorylation of BRI1 and BAK1 (Shan et al., 2008). Considering that AvrPto targets the RLCKs BSK1 and CDG1, which are two direct phosphorylation substrates of BRI1 (Kim et al., 2011; Shan et al., 2008; Tang et al., 2008; Xiang et al., 2011), AvrPto probably inhibits BRI1mediated phosphorylation of these two RLCKs to suppress BRI1 signalling. Collectively, these findings suggest that AvrPto functions as a kinase inhibitor and as a regulatory protein controlling receptor complex formation, to suppress signalling mediated by LRR-RLK receptors.

AvrPto also suppresses Cf-4 signalling and constitutive immunity mediated by Arabidopsis SOBIR1 in N. benthamiana (Chapter 3). Despite the contradicting reports about the interaction between AvrPto and BAK1 (Shan et al., 2008; Xiang et al., 2011; Zhou et al., 2014), an interaction between AvrPto and BAK1 was found (Chapter 3). In addition, AvrPto also interacts with the different SOBIR1 variants of Arabidopsis, tomato and $N$. benthamiana (Chapter 3). However, the interaction of AvrPto with BAK1 and SOBIR1 does not affect the Avr4-induced formation of the Cf-4/SOBIR1/BAK1 complex (Chapter 3). Considering that AvrPto inhibits the kinase activity of its interactors (Xiang et al., 2008), AvrPto is proposed to inhibit the kinase activity of SOBIR1 and BAK1 to suppress Cf-4 signalling (Fig. 1). Alternatively, since AvrPto targets the RLCKs BSK1 and CDG1 (Xiang et al., 2011), AvrPto might also target yet unknown RLCKs functioning downstream of the Cf-4/SOBIR1/BAK1 complex.

The Pst DC3000 T3E HopAO1, which is a tyrosine phosphatase, negatively regulates immune signalling mediated by EFR and FLS2 (Bretz et al., 2003; Macho et al., 2014; Underwood et al., 2007). In addition, HopAO1 directly targets the kinase domains of EFR and FLS2, and suppresses the kinase activity of EFR and, probably also of FLS2 (Macho et al., 2014) (Fig. 1). Another example is the Pst DC3000 T3E HopF2, which is a mono-ADP-ribosyltransferase and suppresses immune responses triggered by 
EFR and FLS2 (Wang et al., 2010c; Wu et al., 2011). HopF2 interacts with BAK1 and probably inhibits its kinase activity through ADP-ribosylation (Fig. 1), resulting in inhibition of flg22-induced BIK1 phosphorylation (Wu et al., 2011; Zhou et al., 2014). Furthermore, the Xanthomonas campestris pv. campestris T3E AvrAC functions as a uridylyl transferase to inhibit the kinase activity of various RLCK VII subfamily members including BIK1 (Fig. 1), thereby suppressing defence responses mediated by EFR and FLS2 (Feng et al., 2012).

T3Es suppress the accumulation of functional cell surface receptor complexes

AvrPtoB is another well-known Pst DC3000 T3E that interacts with FLS2, EFR and BAK1, and possesses E3 ligase activity to ubiquitinate FLS2 and EFR for degradation (Göhre et al., 2008; Shan et al., 2008) (Fig. 1). In addition, AvrPtoB also inhibits the formation of the FLS2/BAK1 complex, and the activation of BAK1 (Cheng et al., 2011; Shan et al., 2008).

The Pst DC3000 T3E HopU1 is a mono-ADP-ribosyltransferase, which inhibits the ligand-induced synthesis of FLS2 and EFR proteins (Fig. 1), through ADP ribosylation of the RNA-binding protein GLYCINE-RICH PROTEIN 7 (GRP7) (Fu et al., 2007; Jeong et al., 2011; Nicaise et al., 2013). GRP7 binds the FLS2 transcript, as well as the FLS2 protein (Nicaise et al., 2013). HopU1 disrupts complex formation of GRP7 with the FLS2 transcript, but not with the FLS2 protein, eventually resulting in a lower abundance of the FLS2 protein (Nicaise et al., 2013).

The Pst DC3000 T3E HopB1 constitutively associates with FLS2 and functions as a serine protease that specifically cleaves activated BAK1, thereby suppressing activation of downstream immune responses (Li et al., 2016b) (Fig. 1). In addition, the P. syringae pv. phaseolicola T3E AvrPphB functions as a cysteine protease to cleave multiple RLCK VII subfamily members including BIK1, thereby suppressing defence responses mediated by FLS2 and EFR (Zhang et al., 2010) (Fig. 1).

\section{Concluding remarks}

Plant cell surface LRR-RLK and LRR-RLP receptors play an important role in responding to extracellular signals through the activation of overlapping, but distinct signalling pathways. Studying the molecular mechanism underlying receptor-triggered responses occurring at any cellular level will benefit our understanding of how plants react to extracellular stimuli. Earlier research revealed that both LRR-RLK and LRRRLP receptors require BAK1 as a regulatory $R L K$, while LRR-RLP receptors additionally require the regulatory RLK SOBIR1 for their functionality. Notably, recent studies on receptor complexes also revealed the involvement of plasma membrane- 
associated and cytoplasmic regulators that either positively or negatively regulate the formation, activation and abundance of functional receptor complexes. These findings have not only led to a much better understanding of how receptor complexes are controlled and activated prior to and upon ligand perception, but also pointed out some similar but also distinct scenarios for the role of regulators in controlling receptor signalling. For instance, BIR2 and BIR3 have similar but yet different functions in regulating signalling mediated by LRR-RLK and LRR-RLP receptors through controlling the association of receptors with their regulatory LRR-RLKs. Notably, the molecular mechanism behind the role of a certain regulator in controlling the activity of different cell surface receptor complexes might be different, as for example PUB12/13 regulate the abundance of the FLS2 and BRI1 proteins in a BAK1-dependent and dependent manner, respectively. More importantly, as the development-regulating ligand-binding receptors ANX1/2 and FER also regulate defence through their direct involvement in LRR-RLK receptor complexes mediating immunity, it will be interesting to study whether ANX1/2 and FER, as well as other malectin-like domain-containing receptors, function as regulatory nodes that balance the trade-off between defence and development. Up till now, our knowledge on the molecular mechanism underlying the regulation of receptor complexes is still limited. It is conceivable that with further research on the general and specific roles of the currently known and additionally characterized regulators that control the formation, activation and abundance of the various cell surface receptor complexes, a new level of complexity of the mechanisms underlying the regulation of receptor complexes will emerge. This will eventually lead to an even better understanding of cell surface receptor networks, which will lay a basis for further engineering of plant immunity with the aim to secure future food production. 



\section{References}

Ade, J., DeYoung, B.J., Golstein, C., and Innes, R.W. (2007). Indirect activation of a plant nucleotide binding site-leucine-rich repeat protein by a bacterial protease. Proc Natl Acad Sci USA 104:253125366.

Afroz, A., Chaudhry, Z., Rashid, U., Ali, G.M., Nazir, F., Iqbal, J., and Khan, M.R. (2011). Enhanced resistance against bacterial wilt in transgenic tomato (Lycopersicon esculentum) lines expressing the Xa21 gene. Plant Cell Tissue Organ Cult 104:227-237.

Albert, I., Böhm, H., Albert, M., Feiler, C.E., Imkampe, J., Wallmeroth, N., Brancato, C., Raaymakers, T.M., Oome, S., Zhang, H., et al. (2015). An RLP23-SOBIR1-BAK1 complex mediates NLPtriggered immunity. Nat Plants 1:15140-15148.

Albert, M., Jehle, A.K., Mueller, K., Eisele, C., Lipschis, M., and Felix, G. (2010). Arabidopsis thaliana pattern recognition receptors for bacterial elongation factor $\mathrm{Tu}$ and flagellin can be combined to form functional chimeric receptors. J Biol Chem 285:19035-19042.

Albrecht, C., Russinova, E., Hecht, V., Baaijens, E., and de Vries, S. (2005). The Arabidopsis thaliana SOMATIC EMBRYOGENESIS RECEPTOR-LIKE KINASES1 and 2 control male sporogenesis. Plant Cell 17:3337-3349.

Amano, Y., Tsubouchi, H., Shinohara, H., Ogawa, M., and Matsubayashi, Y. (2007). Tyrosine-sulfated glycopeptide involved in cellular proliferation and expansion in Arabidopsis. Proc Natl Acad Sci USA 104:18333-18338.

Anne, P., Amiguet-Vercher, A., Brandt, B., Kalmbach, L., Geldner, N., Hothorn, M., and Hardtke, C.S. (2018). CLERK is a novel receptor kinase required for sensing of root-active CLE peptides in Arabidopsis. Development 145:dev162354.

Asai, T., Tena, G., Plotnikova, J., Willmann, M.R., Chiu, W.L., Gomez-Gomez, L., Boller, T., Ausubel, F.M., and Sheen, J. (2002). MAP kinase signalling cascade in Arabidopsis innate immunity. Nature 415:977-983.

Balmuth, A., and Rathjen, J.P. (2007). Genetic and molecular requirements for function of the Pto/Prf effector recognition complex in tomato and Nicotiana benthamiana. Plant J 51:978-990.

Bendahmane, A., Farnham, G., Moffett, P., and Baulcombe, D.C. (2002). Constitutive gain-of-function mutants in a nucleotide binding site-leucine rich repeat protein encoded at the $R x$ locus of potato. Plant J 32:195-204.

Bethke, G., Pecher, P., Eschen-Lippold, L., Tsuda, K., Katagiri, F., Glazebrook, J., Scheel, D., and Lee, J. (2012). Activation of the Arabidopsis thaliana mitogen-activated protein kinase MPK11 by the flagellin-derived elicitor peptide, flg22. Mol Plant Microbe Interact 25:471-480.

Bethke, G., Unthan, T., Uhrig, J.F., Pöschl, Y., Gust, A.A., Scheel, D., and Lee, J. (2009). Flg22 regulates the release of an ethylene response factor substrate from MAP kinase 6 in Arabidopsis thaliana via ethylene signaling. Proc Natl Acad Sci USA 106:8067-8072.

Bi, G., Liebrand, T.W.H., Bye, R.R., Postma, J., van der Burgh, A.M., Robatzek, S., Xu, X., and Joosten, M.H.A.J. (2016). SOBIR1 requires the GxxxG dimerization motif in its transmembrane domain to form constitutive complexes with receptor-like proteins. Mol Plant Pathol 17:96-107.

$\mathrm{Bi}$, G., and Zhou, J.M. (2017). MAP kinase signaling pathways: a hub of plant-microbe interactions. Cell Host Microbe 21:270-273.

Bi, G., Zhou, Z., Wang, W., Li, L., Rao, S., Wu, Y., Zhang, X., Menke, F.L., Chen, S., and Zhou, J.-M. (2018). Receptor-like cytoplasmic kinases directly link diverse pattern recognition receptors to the activation of mitogen-activated protein kinase cascades in Arabidopsis. Plant Cell 30:1543-1561.

Bigeard, J., Colcombet, J., and Hirt, H. (2015). Signaling mechanisms in pattern-triggered immunity (PTI). Mol Plant 8:521-539.

Blaum, B.S., Mazzotta, S., Noldeke, E.R., Halter, T., Madlung, J., Kemmerling, B., and Stehle, T. (2014). Structure of the pseudokinase domain of BIR2, a regulator of BAK1-mediated immune signaling in Arabidopsis. J Struct Biol 186:112-121.

Bleckmann, A., Weidtkamp-Peters, S., Seidel, C.A., and Simon, R. (2010). Stem cell signaling in Arabidopsis requires CRN to localize CLV2 to the plasma membrane. Plant Physiol 152:166-176.

Böhm, H., Albert, I., Fan, L., Reinhard, A., and Nürnberger, T. (2014a). Immune receptor complexes at the plant cell surface. Curr Opin Plant Biol 20:47-54.

Böhm, H., Albert, I., Oome, S., Raaymakers, T.M., van den Ackerveken, G., and Nurnberger, T. (2014b). A conserved peptide pattern from a widespread microbial virulence factor triggers pattern-induced immunity in Arabidopsis. PLoS Pathog 10:e1004491.

Boisson-Dernier, A., Roy, S., Kritsas, K., Grobei, M.A., Jaciubek, M., Schroeder, J.I., and Grossniklaus, U. (2009). Disruption of the pollen-expressed FERONIA homologs ANXUR1 and ANXUR2 triggers pollen tube discharge. Development 136:3279-3288.

Boller, T., and Felix, G. (2009). A renaissance of elicitors: perception of microbe-associated molecular 
patterns and danger signals by pattern-recognition receptors. Annu Rev Plant Biol 60:379-406.

Bolton, M.D., van Esse, H.P., Vossen, J.H., de Jonge, R., Stergiopoulos, I., Stulemeijer, I.J., van den Berg, G.C., Borras-Hidalgo, O., Dekker, H.L., de Koster, C.G., et al. (2008). The novel Cladosporium fulvum lysin motif effector Ecp6 is a virulence factor with orthologues in other fungal species. Mol Microbiol 69:119-136.

Bommert, P., Je, B.I., Goldshmidt, A., and Jackson, D. (2013). The maize Ga gene COMPACT PLANT2 functions in CLAVATA signalling to control shoot meristem size. Nature 502:555-558.

Bommert, P., Lunde, C., Nardmann, J., Vollbrecht, E., Running, M., Jackson, D., Hake, S., and Werr, W. (2005). thick tassel dwarf1 encodes a putative maize ortholog of the Arabidopsis CLAVATA1 leucine-rich repeat receptor-like kinase. Development 132:1235-1245.

Boudsocq, M., Willmann, M.R., McCormack, M., Lee, H., Shan, L., He, P., Bush, J., Cheng, S.H., and Sheen, J. (2010). Differential innate immune signalling via $\mathrm{Ca}^{2+}$ sensor protein kinases. Nature 464:418-422.

Boutrot, F., and Zipfel, C. (2017). Function, discovery, and exploitation of plant pattern recognition receptors for broad-spectrum disease resistance. Annu Rev Phytopathol 55:257-286.

Bretz, J.R., Mock, N.M., Charity, J.C., Zeyad, S., Baker, C.J., and Hutcheson, S.W. (2003). A translocated protein tyrosine phosphatase of Pseudomonas syringae pv. tomato DC3000 modulates plant defence response to infection. Mol Microbiol 49:389-400.

Brock, A.K., Willmann, R., Kolb, D., Grefen, L., Lajunen, H.M., Bethke, G., Lee, J., Nurnberger, T., and Gust, A.A. (2010). The Arabidopsis mitogen-activated protein kinase phosphatase PP2C5 affects seed germination, stomatal aperture, and abscisic acid-inducible gene expression. Plant Physiol 153:1098-1111.

Brutus, A., Sicilia, F., Macone, A., Cervone, F., and De Lorenzo, G. (2010). A domain swap approach reveals a role of the plant wall-associated kinase 1 (WAK1) as a receptor of oligogalacturonides. Proc Natl Acad Sci USA 107:9452-9457.

Bucherl, C.A., Jarsch, I.K., Schudoma, C., Segonzac, C., Mbengue, M., Robatzek, S., MacLean, D., Ott, T., and Zipfel, C. (2017). Plant immune and growth receptors share common signalling components but localise to distinct plasma membrane nanodomains. eLife 6:e25114.

Burkart, R.C., and Stahl, Y. (2017). Dynamic complexity: plant receptor complexes at the plasma membrane. Curr Opin Plant Biol 40:15-21.

Burr, C.A., Leslie, M.E., Orlowski, S.K., Chen, I., Wright, C.E., Daniels, M.J., and Liljegren, S.J. (2011). CAST AWAY, a membrane-associated receptor-like kinase, inhibits organ abscission in Arabidopsis. Plant Physiol 156:1837-1850.

Butenko, M.A., Patterson, S.E., Grini, P.E., Stenvik, G.E., Amundsen, S.S., Mandal, A., and Aalen, R.B. (2003). INFLORESCENCE DEFICIENT IN ABSCISSION controls floral organ abscission in Arabidopsis and identifies a novel family of putative ligands in plants. Plant Cell 15:2296-2307.

Büttner, D. (2016). Behind the lines-actions of bacterial type III effector proteins in plant cells. FEMS Microbiol Rev 40:894-937.

Büttner, D., and He, S.Y. (2009). Type III protein secretion in plant pathogenic bacteria. Plant Physiol 150:1656-1664.

Casamitjana-Martinez, E., Hofhuis, H.F., Xu, J., Liu, C.M., Heidstra, R., and Scheres, B. (2003). Rootspecific CLE19 overexpression and the sol1/2 suppressors implicate a CLV-like pathway in the control of Arabidopsis root meristem maintenance. Curr Biol 13:1435-1441.

Castaneda-Ojeda, M.P., Moreno-Perez, A., Ramos, C., and Lopez-Solanilla, E. (2017). Suppression of plant immune responses by the Pseudomonas savastanoi pv. savastanoi NCPPB 3335 type III effector tyrosine phosphatases HopAO1 and HopAO2. Front Plant Sci 8:680.

Catanzariti, A.M., Do, H.T., Bru, P., de Sain, M., Thatcher, L.F., Rep, M., and Jones, D.A. (2017). The tomato I gene for Fusarium wilt resistance encodes an atypical leucine-rich repeat receptor-like protein whose function is nevertheless dependent on SOBIR1 and SERK3/BAK1. Plant $\mathrm{J}$ 89:1195-1209.

Chakrabarti, A., Velusamy, T., Tee, C.Y., and Jones, D.A. (2016). A mutational analysis of the cytosolic domain of the tomato Cf-9 disease-resistance protein shows that membrane-proximal residues are important for Avr9-dependent necrosis. Mol Plant Pathol 17:565-576.

Chen, L., and Hellmann, H. (2013). Plant E3 ligases: flexible enzymes in a sessile world. Mol Plant 6:1388-1404.

Chen, W., and Ow, D.W. (2017). Precise, flexible and affordable gene stacking for crop improvement. Bioengineered 8:451-456.

Chen, X., Chern, M., Canlas, P.E., Ruan, D., Jiang, C., and Ronald, P.C. (2010). An ATPase promotes autophosphorylation of the pattern recognition receptor XA21 and inhibits XA21-mediated immunity. Proc Natl Acad Sci USA 107:8029-8034. 
Chen, X., Zuo, S., Schwessinger, B., Chern, M., Canlas, P.E., Ruan, D., Zhou, X., Wang, J., Daudi, A., Petzold, C.J., et al. (2014). An XA21-associated kinase (OsSERK2) regulates immunity mediated by the XA21 and XA3 immune receptors. Mol Plant 7:874-892.

Cheng, S.H., Willmann, M.R., Chen, H.C., and Sheen, J. (2002). Calcium signaling through protein kinases. The Arabidopsis calcium-dependent protein kinase gene family. Plant Physiol 129:469485.

Cheng, W., Munkvold, K.R., Gao, H., Mathieu, J., Schwizer, S., Wang, S., Yan, Y.B., Wang, J., Martin, G.B., and Chai, J. (2011). Structural analysis of Pseudomonas syringae AvrPtoB bound to host BAK1 reveals two similar kinase-interacting domains in a type III Effector. Cell Host Microbe 10:616-626.

Chinchilla, D., Bauer, Z., Regenass, M., Boller, T., and Felix, G. (2006). The Arabidopsis receptor kinase FLS2 binds flg22 and determines the specificity of flagellin perception. Plant Cell 18:465-476.

Chinchilla, D., Zipfel, C., Robatzek, S., Kemmerling, B., Nurnberger, T., Jones, J.D.G., Felix, G., and Boller, T. (2007). A flagellin-induced complex of the receptor FLS2 and BAK1 initiates plant defence. Nature 448:497-500.

Cho, S.K., Larue, C.T., Chevalier, D., Wang, H., Jinn, T.L., Zhang, S., and Walker, J.C. (2008). Regulation of floral organ abscission in Arabidopsis thaliana. Proc Natl Acad Sci USA 105:1562915634.

Clark, S.E., Running, M.P., and Meyerowitz, E.M. (1993). CLAVATA1, a regulator of meristem and flower development in Arabidopsis. Development 119:397-418.

Clark, S.E., Running, M.P., and Meyerowitz, E.M. (1995). CLAVATA3 is a specific regulator of shoot and floral meristem development affecting the same processes as CLAVATA1. Development 121:2057-2067.

Clark, S.E., Williams, R.W., and Meyerowitz, E.M. (1997). The CLAVATA1 gene encodes a putative receptor kinase that controls shoot and floral meristem size in Arabidopsis. Cell 89:575-585.

Colcombet, J., Boisson-Dernier, A., Ros-Palau, R., Vera, C.E., and Schroeder, J.I. (2005). Arabidopsis SOMATIC EMBRYOGENESIS RECEPTOR KINASES1 and 2 are essential for tapetum development and microspore maturation. Plant Cell 17:3350-3361.

Cook, D.E., Mesarich, C.H., and Thomma, B.P.H.J. (2015). Understanding plant immunity as a surveillance system to detect invasion. Annu Rev Phytopathol 53:541-563.

Couto, D., Niebergall, R., Liang, X., Bucherl, C.A., Sklenar, J., Macho, A.P., Ntoukakis, V., Derbyshire, P., Altenbach, D., Maclean, D., et al. (2016). The Arabidopsis protein phosphatase PP2C38 negatively regulates the central immune kinase BIK1. PLoS Pathog 12:e1005811.

Couto, D., and Zipfel, C. (2016). Regulation of pattern recognition receptor signalling in plants. Nat Rev Immunol 16:537-552.

Cui, F., Sun, W., and Kong, X. (2018). RLCKs bridge plant immune receptors and MAPK cascades. Trends Plant Sci 23:1039-1041.

Cui, H., Xiang, T., and Zhou, J.M. (2009). Plant immunity: a lesson from pathogenic bacterial effector proteins. Cell Microbiol 11:1453-1461.

Cunnac, S., Lindeberg, M., and Collmer, A. (2009). Pseudomonas syringae type III secretion system effectors: repertoires in search of functions. Curr Opin Microbiol 12:53-60.

Dangl, J.L., Horvath, D.M., and Staskawicz, B.J. (2013). Pivoting the plant immune system from dissection to deployment. Science 341:746-751.

de Jong, C.F., Laxalt, A.M., Bargmann, B.O., de Wit, P.J., Joosten, M.H., and Munnik, T. (2004). Phosphatidic acid accumulation is an early response in the Cf-4/Avr4 interaction. Plant J 39:1-12.

de Jonge, R., van Esse, H.P., Kombrink, A., Shinya, T., Desaki, Y., Bours, R., van der Krol, S., Shibuya, N., Joosten, M.H., and Thomma, B.P. (2010). Conserved fungal LysM effector Ecp6 prevents chitin-triggered immunity in plants. Science 329:953-955.

de Jonge, R., van Esse, H.P., Maruthachalam, K., Bolton, M.D., Santhanam, P., Saber, M.K., Zhang, Z., Usami, T., Lievens, B., Subbarao, K.V., et al. (2012). Tomato immune receptor Ve1 recognizes effector of multiple fungal pathogens uncovered by genome and RNA sequencing. Proc Natl Acad Sci USA 109:5110-5115.

De Lorenzo, G., Brutus, A., Savatin, D.V., Sicilia, F., and Cervone, F. (2011). Engineering plant resistance by constructing chimeric receptors that recognize damage-associated molecular patterns (DAMPs). FEBS Lett 585:1521-1528.

de Wit, P.J. (2016). Cladosporium fulvum effectors: Weapons in the arms race with tomato. Annu Rev Phytopathol 54:1-23.

Deng, Q., and Barbieri, J.T. (2008). Molecular mechanisms of the cytotoxicity of ADP-ribosylating toxins. Annu Rev Microbiol 62:271-288.

Depuydt, S., Rodriguez-Villalon, A., Santuari, L., Wyser-Rmili, C., Ragni, L., and Hardtke, C.S. (2013). 


\section{References}

Suppression of Arabidopsis protophloem differentiation and root meristem growth by CLE45 requires the receptor-like kinase BAM3. Proc Natl Acad Sci USA 110:7074-7079.

DeYoung, B.J., Bickle, K.L., Schrage, K.J., Muskett, P., Patel, K., and Clark, S.E. (2006). The CLAVATA1related BAM1, BAM2 and BAM3 receptor kinase-like proteins are required for meristem function in Arabidopsis. Plant J 45:1-16.

Ding, L., Pandey, S., and Assmann, S.M. (2008). Arabidopsis extra-large G proteins (XLGs) regulate root morphogenesis. Plant J 53:248-263.

Dixon, M.S., Hatzixanthis, K., Jones, D.A., Harrison, K., and Jones, J.D. (1998). The tomato Cf-5 disease resistance gene and six homologs show pronounced allelic variation in leucine-rich repeat copy number. Plant Cell 10:1915-1925.

Dixon, M.S., Jones, D.A., Keddie, J.S., Thomas, C.M., Harrison, K., and Jones, J.D. (1996). The tomato Cf-2 disease resistance locus comprises two functional genes encoding leucine-rich repeat proteins. Cell 84:451-459.

Dodds, P.N., and Rathjen, J.P. (2010). Plant immunity: towards an integrated view of plant-pathogen interactions. Nat Rev Genet 11:539-548.

Domazakis, E., Wouters, D., Visser, R.G.F., Kamoun, S., Joosten, M.H.A.J., and Vleeshouwers, V.G.A.A. (2018). The ELR-SOBIR1 complex functions as a two-component receptor-like kinase to mount defense against Phytophthora infestans. Mol Plant Microbe Interact 31:795-802.

Dong, J., Xiao, F., Fan, F., Gu, L., Cang, H., Martin, G.B., and Chai, J. (2009). Crystal structure of the complex between Pseudomonas effector AvrPtoB and the tomato Pto kinase reveals both a shared and a unique interface compared with AvrPto-Pto. Plant Cell 21:1846-1859.

Du, J., Verzaux, E., Chaparro-Garcia, A., Bijsterbosch, G., Keizer, L.C., Zhou, J., Liebrand, T.W., Xie, C., Govers, F., Robatzek, S., et al. (2015). Elicitin recognition confers enhanced resistance to Phytophthora infestans in potato. Nat Plants 1:15034.

Dubiella, U., Seybold, H., Durian, G., Komander, E., Lassig, R., Witte, C.P., Schulze, W.X., and Romeis, T. (2013). Calcium-dependent protein kinase/NADPH oxidase activation circuit is required for rapid defense signal propagation. Proc Natl Acad Sci USA 110:8744-8749.

Durbak, A.R., and Tax, F.E. (2011). CLAVATA signaling pathway receptors of Arabidopsis regulate cell proliferation in fruit organ formation as well as in meristems. Genetics 189:177-194.

Durrant, W.E., Rowland, O., Piedras, P., Hammond-Kosack, K.E., and Jones, J.D.G. (2000). cDNAAFLP reveals a striking overlap in race-specific resistance and wound response gene expression profiles. Plant Cell 12:963-977.

Escobar-Restrepo, J.M., Huck, N., Kessler, S., Gagliardini, V., Gheyselinck, J., Yang, W.C., and Grossniklaus, U. (2007). The FERONIA receptor-like kinase mediates male-female interactions during pollen tube reception. Science 317:656-660.

Feilner, T., Hultschig, C., Lee, J., Meyer, S., Immink, R.G., Koenig, A., Possling, A., Seitz, H., Beveridge, A., and Scheel, D. (2005). High throughput identification of potential Arabidopsis mitogenactivated protein kinases substrates. Mol Cell Proteom 4:1558-1568.

Felix, G., Duran, J.D., Volko, S., and Boller, T. (1999). Plants have a sensitive perception system for the most conserved domain of bacterial flagellin. Plant J 18:265-276.

Feng, F., Yang, F., Rong, W., Wu, X., Zhang, J., Chen, S., He, C., and Zhou, J.M. (2012). A Xanthomonas uridine $5^{\prime}$-monophosphate transferase inhibits plant immune kinases. Nature 485:114-118.

Ferrari, S., Galletti, R., Vairo, D., Cervone, F., and De Lorenzo, G. (2006). Antisense expression of the Arabidopsis thaliana AtPGIP1 gene reduces polygalacturonase-inhibiting protein accumulation and enhances susceptibility to Botrytis cinerea. Mol Plant Microbe Interact 19:931-936.

Fiers, M., Golemiec, E., van der Schors, R., van der Geest, L., Li, K.W., Stiekema, W.J., and Liu, C.M. (2006). The CLAVATA3/ESR motif of CLAVATA3 is functionally independent from the nonconserved flanking sequences. Plant Physiol 141:1284-1292.

Fiers, M., Golemiec, E., Xu, J., van der Geest, L., Heidstra, R., Stiekema, W., and Liu, C.M. (2005). The 14-amino acid CLV3, CLE19, and CLE40 peptides trigger consumption of the root meristem in Arabidopsis through a CLAVATA2-dependent pathway. Plant Cell 17:2542-2553.

Fisher, K., and Turner, S. (2007). PXY, a receptor-like kinase essential for maintaining polarity during plant vascular-tissue development. Curr Biol 17:1061-1066.

Fletcher, J.C., Brand, U., Running, M.P., Simon, R., and Meyerowitz, E.M. (1999). Signaling of cell fate decisions by CLAVATA3 in Arabidopsis shoot meristems. Science 283:1911-1914.

Franck, C.M., Westermann, J., and Boisson-Dernier, A. (2018). Plant malectin-like receptor kinases: From cell wall integrity to immunity and beyond. Annu Rev Plant Biol 69:301-328.

Frei dit Frey, N., Mbengue, M., Kwaaitaal, M., Nitsch, L., Altenbach, D., Haweker, H., Lonzano-Duran, R., Njo, M.F., Beeckman, T., and Huettel, B. (2012). Plasma membrane calcium ATPases are important components of receptor-mediated signalling in plant immune responses and 
development. Plant Physiol 159:798-809.

Fu, Z.Q., Guo, M., Jeong, B.R., Tian, F., Elthon, T.E., Cerny, R.L., Staiger, D., and Alfano, J.R. (2007). A type III effector ADP-ribosylates RNA-binding proteins and quells plant immunity. Nature 447:284-288.

Furlan, G., Nakagami, H., Eschen-Lippold, L., Jiang, X., Majovsky, P., Kowarschik, K., Hoehenwarter, W., Lee, J., and Trujillo, M. (2017). Changes in PUB22 ubiquitination modes triggered by MITOGEN-ACTIVATED PROTEIN KINASE3 dampen the immune response. Plant Cell 29:726745 .

Gabriëls, S.H., Takken, F.L., Vossen, J.H., de Jong, C.F., Liu, Q., Turk, S.C., Wachowski, L.K., Peters, J., Witsenboer, H.M., de Wit, P.J., et al. (2006). cDNA-AFLP combined with functional analysis reveals novel genes involved in the hypersensitive response. Mol Plant Microbe Interact 19:567576.

Galletti, R., Ferrari, S., and De Lorenzo, G. (2011). Arabidopsis MPK3 and MPK6 play different roles in basal and oligogalacturonide- or flagellin-induced resistance against Botrytis cinerea. Plant Physiol 157:804-814.

Gao, M., Liu, J., Bi, D., Zhang, Z., Cheng, F., Chen, S., and Zhang, Y. (2008). MEKK1, MKK1/MKK2 and MPK4 function together in a mitogen-activated protein kinase cascade to regulate innate immunity in plants. Cell Res 18:1190-1198.

Gao, M., Wang, X., Wang, D., Xu, F., Ding, X., Zhang, Z., Bi, D., Cheng, Y., Chen, S., Li, X., et al. (2009). Regulation of cell death and innate immunity by two receptor-like kinases in Arabidopsis. Cell Host Microbe 6:34-44.

Ge, Z., Bergonci, T., Zhao, Y., Zou, Y., Du, S., Liu, M.C., Luo, X., Ruan, H., Garcia-Valencia, L.E., Zhong, S., et al. (2017). Arabidopsis pollen tube integrity and sperm release are regulated by RALFmediated signaling. Science 358:1596-1600.

Geissler, K., Eschen-Lippold, L., Naumann, K., Schneeberger, K., Weigel, D., Scheel, D., Rosahl, S., and Westphal, L. (2015). Mutations in the EDR1 gene alter the response of Arabidopsis thaliana to Phytophthora infestans and the bacterial PAMPs flg22 and elf18. Mol Plant Microbe Interact 28:122-133.

Gilroy, E.M., Taylor, R.M., Hein, I., Boevink, P., Sadanandom, A., and Birch, P.R. (2011). CMPG1dependent cell death follows perception of diverse pathogen elicitors at the host plasma membrane and is suppressed by Phytophthora infestans RXLR effector AVR3a. New Phytol 190:653-666.

Gimenez-lbanez, S., Hann, D.R., Ntoukakis, V., Petutschnig, E., Lipka, V., and Rathjen, J.P. (2009). AvrPtoB targets the LysM receptor kinase CERK1 to promote bacterial virulence on plants. Curr Biol 19:423-429.

Giska, F., and Martin, G.B. (2019). PP2C phosphatase Pic1 negatively regulates phosphorylation status of Pti1 b kinase, a regulator of flagellin-triggered immunity in tomato. Biochem $\mathrm{J} 476: 1621-1635$.

Göhre, V., Spallek, T., Haweker, H., Mersmann, S., Mentzel, T., Boller, T., de Torres, M., Mansfield, J.W., and Robatzek, S. (2008). Plant pattern-recognition receptor FLS2 is directed for degradation by the bacterial ubiquitin ligase AvrPtoB. Curr Biol 18:1824-1832.

Gómez-Gómez, L., Bauer, Z., and Boller, T. (2001). Both the extracellular leucine-rich repeat domain and the kinase activity of FLS2 are required for flagellin binding and signaling in Arabidopsis. Plant Cell 13:1155-1163.

Gómez-Gómez, L., and Boller, T. (2000). FLS2: an LRR receptor-like kinase involved in the perception of the bacterial elicitor flagellin in Arabidopsis. Mol Cell 5:1003-1011.

Gómez, B.G., Lozano-Durán, R., and Wolf, S. (2019). Phosphorylation-dependent routing of RLP44 towards brassinosteroid or phytosulfokine signalling. bioRxiv:527754.

González-Lamothe, R., Tsitsigiannis, D.I., Ludwig, A.A., Panicot, M., Shirasu, K., and Jones, J.D. (2006). The U-box protein CMPG1 is required for efficient activation of defense mechanisms triggered by multiple resistance genes in tobacco and tomato. Plant Cell 18:1067-1083.

Gopalan, S., Wei, W., and He, S.Y. (1996). hrp gene-dependent induction of hin1: a plant gene activated rapidly by both harpins and the avrPto gene-mediated signal. Plant J 10:591-600.

Großeholz, R., Feldman-Salit, A., Wanke, F., Schulze, S., Glockner, N., Kemmerling, B., Harter, K., and Kummer, U. (2019). Specifying the role of BAK1-interacting receptor-like kinase 3 in brassinosteroid signaling. J Integr Plant Biol:doi.org/10.1111/jipb.12803.

Gust, A.A., and Felix, G. (2014). Receptor like proteins associate with SOBIR1-type of adaptors to form bimolecular receptor kinases. Curr Opin Plant Biol 21:104-111.

Gust, A.A., Pruitt, R., and Nurnberger, T. (2017). Sensing danger: Key to activating plant immunity. Trends Plant Sci 22:779-791.

Guzmán-Benito, I., Donaire, L., Amorim-Silva, V., Vallarino, J.G., Esteban, A., Wierzbicki, A.T., Ruiz- 


\section{References}

Ferrer, V., and Llave, C. (2019). The immune repressor BIR1 contributes to antiviral defense and undergoes transcriptional and post-transcriptional regulation during viral infections. New Phytol 224:421-438

Halpin, C. (2005). Gene stacking in transgenic plants-the challenge for 21 st century plant biotechnology. Plant Biotechnol J 3:141-155.

Halter, T., Imkampe, J., Blaum, B.S., Stehle, T., and Kemmerling, B. (2014a). BIR2 affects complex formation of BAK1 with ligand binding receptors in plant defense. Plant Signal Behav 9:134-143.

Halter, T., Imkampe, J., Mazzotta, S., Wierzba, M., Postel, S., Bucherl, C., Kiefer, C., Stahl, M., Chinchilla, D., Wang, X., et al. (2014b). The leucine-rich repeat receptor kinase BIR2 is a negative regulator of BAK1 in plant immunity. Curr Biol 24:134-143.

Hammond-Kosack, K.E., Harrison, K., and Jones, J.D. (1994). Developmentally regulated cell death on expression of the fungal avirulence gene Avr9 in tomato seedlings carrying the disease-resistance gene Cf-9. Proc Natl Acad Sci USA 91:10445-10449.

Hammond-Kosack, K.E., Tang, S., Harrison, K., and Jones, J.D.G. (1998). The tomato Cf-9 disease resistance gene functions in tobacco and potato to confer responsiveness to the fungal avirulence gene product Avr9. Plant Cell 10:1251-1266.

Hann, D.R., and Rathjen, J.P. (2007). Early events in the pathogenicity of Pseudomonas syringae on Nicotiana benthamiana. Plant J 49:607-618.

Hara, K., Kajita, R., Torii, K.U., Bergmann, D.C., and Kakimoto, T. (2007). The secretory peptide gene EPF1 enforces the stomatal one-cell-spacing rule. Genes Dev 21:1720-1725.

Harper, J.F., Hong, B., Hwang, I., Guo, H.Q., Stoddard, R., Huang, J.F., Palmgren, M.G., and Sze, H. (1998). A novel calmodulin-regulated $\mathrm{Ca}^{2+}$-ATPase (ACA2) from Arabidopsis with an N-terminal autoinhibitory domain. J Biol Chem 273:1099-1106.

Haruta, M., Sabat, G., Stecker, K., Minkoff, B.B., and Sussman, M.R. (2014). A peptide hormone and its receptor protein kinase regulate plant cell expansion. Science 343:408-411.

Hazak, O., Brandt, B., Cattaneo, P., Santiago, J., Rodriguez-Villalon, A., Hothorn, M., and Hardtke, C.S. (2017). Perception of root-active CLE peptides requires CORYNE function in the phloem vasculature. EMBO Rep 18:1367-1381.

He, K., Gou, X., Yuan, T., Lin, H., Asami, T., Yoshida, S., Russell, S.D., and Li, J. (2007). BAK1 and BKK1 regulate brassinosteroid-dependent growth and brassinosteroid-independent cell-death pathways. Curr Biol 17:1109-1115.

He, P., Shan, L., Lin, N.C., Martin, G.B., Kemmerling, B., Nurnberger, T., and Sheen, J. (2006). Specific bacterial suppressors of MAMP signaling upstream of MAPKKK in Arabidopsis innate immunity. Cell 125:563-575.

He, Q., McLellan, H., Boevink, P.C., Sadanandom, A., Xie, C., Birch, P.R., and Tian, Z. (2015). U-box E3 ubiquitin ligase PUB17 acts in the nucleus to promote specific immune pathways triggered by Phytophthora infestans. J Exp Bot 66:3189-3199.

He, Z., Wang, Z., Li, J., Zhu, Q., Lamb, C., Ronald, P., and Chory, J. (2000). Perception of brassinosteroids by the extracellular domain of the receptor kinase BRI1. Science 288:2360-2363.

Heese, A., Hann, D.R., Gimenez-Ibanez, S., Jones, A.M., He, K., Li, J., Schroeder, J.I., Peck, S.C., and Rathjen, J.P. (2007). The receptor-like kinase SERK3/BAK1 is a central regulator of innate immunity in plants. Proc Natl Acad Sci USA 104:12217-12222.

Hegenauer, V., Furst, U., Kaiser, B., Smoker, M., Zipfel, C., Felix, G., Stahl, M., and Albert, M. (2016). Detection of the plant parasite Cuscuta reflexa by a tomato cell surface receptor. Science 353:478-481.

Higuchi, R., Krummel, B., and Saiki, R.K. (1988). A general method of in vitro preparation and specific mutagenesis of DNA fragments: study of protein and DNA interactions. Nucleic Acids Res 16:7351-7367.

Hind, S.R., Strickler, S.R., Boyle, P.C., Dunham, D.M., Bao, Z., O'Doherty, I.M., Baccile, J.A., Hoki, J.S., Viox, E.G., Clarke, C.R., et al. (2016). Tomato receptor FLAGELLIN-SENSING 3 binds flgll-28 and activates the plant immune system. Nat Plants 2:16128.

Hirakawa, Y., Shinohara, H., Kondo, Y., Inoue, A., Nakanomyo, I., Ogawa, M., Sawa, S., Ohashi-Ito, K., Matsubayashi, Y., and Fukuda, H. (2008). Non-cell-autonomous control of vascular stem cell fate by a CLE peptide/receptor system. Proc Natl Acad Sci USA 105:15208-15213.

Hobe, M., Muller, R., Grunewald, M., Brand, U., and Simon, R. (2003). Loss of CLE40, a protein functionally equivalent to the stem cell restricting signal CLV3, enhances root waving in Arabidopsis. Dev Genes Evol 213:371-381.

Hohmann, U., and Hothorn, M. (2019). Crystal structure of the leucine-rich repeat ectodomain of the plant immune receptor kinase SOBIR1. Acta Crystallogr D Struct Biol 75:488-497.

Hohmann, U., Lau, K., and Hothorn, M. (2017). The structural basis of ligand perception and signal 
activation by receptor kinases. Annu Rev Plant Biol 68:109-137.

Hohmann, U., Nicolet, J., Moretti, A., Hothorn, L.A., and Hothorn, M. (2018). The SERK3 elongated allele defines a role for BIR ectodomains in brassinosteroid signalling. Nat Plants 4:345-351.

Hok, S., Danchin, E.G., Allasia, V., Panabieres, F., Attard, A., and Keller, H. (2011). An Arabidopsis (malectin-like) leucine-rich repeat receptor-like kinase contributes to downy mildew disease. Plant Cell Environ 34:1944-1957.

Holton, N., Nekrasov, V., Ronald, P.C., and Zipfel, C. (2015). The phylogenetically-related pattern recognition receptors EFR and XA21 recruit similar immune signaling components in monocots and dicots. PLoS Pathog 11:e1004602.

Holzwart, E., Huerta, A.I., Glockner, N., Garnelo Gomez, B., Wanke, F., Augustin, S., Askani, J.C., Schurholz, A.K., Harter, K., and Wolf, S. (2018). BRI1 controls vascular cell fate in the Arabidopsis root through RLP44 and phytosulfokine signaling. Proc Natl Acad Sci USA 115:11838-11843.

Horsch, R.B., Fraley, R.T., Rogers, S.G., Klee, H.J., Fry, J., Hinchee, M.A., and Shah, D.S. (1988). Agrobacterium-mediated gene transfer to plants; engineering tolerance to glyphosate. lowa State J Res 62:487-502.

Hothorn, M., Belkhadir, Y., Dreux, M., Dabi, T., Noel, J.P., Wilson, I.A., and Chory, J. (2011). Structural basis of steroid hormone perception by the receptor kinase BRI1. Nature 474:467-471.

Hou, S., Wang, X., Chen, D., Yang, X., Wang, M., Turra, D., Di Pietro, A., and Zhang, W. (2014). The secreted peptide PIP1 amplifies immunity through receptor-like kinase 7. PLoS Pathog 10:e1004331.

Hu, C., Zhu, Y., Cui, Y., Cheng, K., Liang, W., Wei, Z., Zhu, M., Yin, H., Zeng, L., Xiao, Y., et al. (2018). A group of receptor kinases are essential for CLAVATA signalling to maintain stem cell homeostasis. Nat Plants 4:205-211.

Huang, S., Nie, S., Wang, S., Liu, J., Zhang, Y., and Wang, X. (2017). SIBIR3 negatively regulates PAMP responses and cell death in tomato. Int J Mol Sci 18:1966.

Huck, N., Moore, J.M., Federer, M., and Grossniklaus, U. (2003). The Arabidopsis mutant feronia disrupts the female gametophytic control of pollen tube reception. Development 130:2149-2159.

Hunt, L., and Gray, J.E. (2009). The signaling peptide EPF2 controls asymmetric cell divisions during stomatal development. Curr Biol 19:864-869.

Hutten, S.J., Hamers, D.S., Aan den Toorn, M., van Esse, W., Nolles, A., Bucherl, C.A., de Vries, S.C., Hohlbein, J., and Borst, J.W. (2017). Visualization of BRI1 and SERK3/BAK1 nanoclusters in Arabidopsis roots. PLoS One 12:e0169905.

Hwang, I., Sze, H., and Harper, J.F. (2000). A calcium-dependent protein kinase can inhibit a calmodulinstimulated $\mathrm{Ca}^{2+}$ pump (ACA2) located in the endoplasmic reticulum of Arabidopsis. Proc Natl Acad Sci USA 97:6224-6229.

Imkampe, J., Halter, T., Huang, S., Schulze, S., Mazzotta, S., Schmidt, N., Manstretta, R., Postel, S., Wierzba, M., Yang, Y., et al. (2017). The Arabidopsis leucine-rich repeat receptor kinase BIR3 negatively regulates BAK1 receptor complex formation and stabilizes BAK1. Plant Cell 29:22852303.

Ishida, T., Tabata, R., Yamada, M., Aida, M., Mitsumasu, K., Fujiwara, M., Yamaguchi, K., Shigenobu, S., Higuchi, M., Tsuji, H., et al. (2014). Heterotrimeric G proteins control stem cell proliferation through CLAVATA signaling in Arabidopsis. EMBO Rep 15:1202-1209.

Ito, Y., Nakanomyo, I., Motose, H., Iwamoto, K., Sawa, S., Dohmae, N., and Fukuda, H. (2006). DodecaCLE peptides as suppressors of plant stem cell differentiation. Science 313:842-845.

Jaillais, Y., Hothorn, M., Belkhadir, Y., Dabi, T., Nimchuk, Z.L., Meyerowitz, E.M., and Chory, J. (2011). Tyrosine phosphorylation controls brassinosteroid receptor activation by triggering membrane release of its kinase inhibitor. Genes Dev 25:232-237.

Jamieson, P.A., Shan, L., and He, P. (2018). Plant cell surface molecular cypher: Receptor-like proteins and their roles in immunity and development. Plant Sci 274:242-251.

Je, B.I., Xu, F., Wu, Q., Liu, L., Meeley, R., Gallagher, J.P., Corcilius, L., Payne, R.J., Bartlett, M.E., and Jackson, D. (2018). The CLAVATA receptor FASCIATED EAR2 responds to distinct CLE peptides by signaling through two downstream effectors. eLife 7:e35673.

Jehle, A.K., Furst, U., Lipschis, M., Albert, M., and Felix, G. (2013a). Perception of the novel MAMP eMax from different Xanthomonas species requires the Arabidopsis receptor-like protein ReMAX and the receptor kinase SOBIR. Plant Signal Behav 8:e27408.

Jehle, A.K., Lipschis, M., Albert, M., Fallahzadeh-Mamaghani, V., Furst, U., Mueller, K., and Felix, G. (2013b). The receptor-like protein ReMAX of Arabidopsis detects the Microbe-Associated Molecular Pattern eMax from Xanthomonas. Plant Cell 25:2330-2340.

Jeong, B.R., Lin, Y., Joe, A., Guo, M., Korneli, C., Yang, H., Wang, P., Yu, M., Cerny, R.L., Staiger, D., et al. (2011). Structure function analysis of an ADP-ribosyltransferase type III effector and its RNA- 


\section{References}

binding target in plant immunity. J Biol Chem 286:43272-43281.

Jeong, S., Trotochaud, A.E., and Clark, S.E. (1999). The Arabidopsis CLAVATA2 gene encodes a receptor-like protein required for the stability of the CLAVATA1 receptor-like kinase. Plant Cell 11:1925-1934.

Jeworutzki, E., Roelfsema, M.R., Anschutz, U., Krol, E., Elzenga, J.T., Felix, G., Boller, T., Hedrich, R., and Becker, D. (2010). Early signaling through the Arabidopsis pattern recognition receptors FLS2 and EFR involves $\mathrm{Ca}^{2+}$-associated opening of plasma membrane anion channels. Plant $\mathrm{J} 62: 367$ 378.

Jones, D.A., Thomas, C.M., Hammond-Kosack, K.E., Balint-Kurti, P.J., and Jones, J.D.G. (1994). Isolation of the tomato $\mathrm{Cf}-9$ gene for resistance to Cladosporium fulvum by transposon tagging. Science 266:789-793.

Jones, J.D., and Dangl, J.L. (2006). The plant immune system. Nature 444:323-329.

Joosten, M.H.A.J., Cozijnsen, T.J., and de Wit, P.J.G.M. (1994). Host resistance to a fungal tomato pathogen lost by a single base-pair change in an avirulence gene. Nature 367:384-386.

Joosten, M.H.A.J., Vogelsang, R., Cozijnsen, T.J., Verberne, M.C., and de Wit, P.J.G.M. (1997). The biotrophic fungus Cladosporium fulvum circumvents Cf-4-mediated resistance by producing unstable AVR4 elicitors. Plant Cell 9:367-379.

Jun, J.H., Fiume, E., and Fletcher, J.C. (2008). The CLE family of plant polypeptide signaling molecules. Cell Mol Life Sci 65:743-755.

Kadota, Y., Liebrand, T.W.H., Goto, Y., Sklenar, J., Derbyshire, P., Menke, F.L.H., Torres, M.A., Molina, A., Zipfel, C., Coaker, G., et al. (2019). Quantitative phosphoproteomic analysis reveals common regulatory mechanisms between effector- and PAMP-triggered immunity in plants. New Phytol 221:2160-2175.

Kadota, Y., Sklenar, J., Derbyshire, P., Stransfeld, L., Asai, S., Ntoukakis, V., Jones, J.D., Shirasu, K., Menke, F., Jones, A., et al. (2014). Direct regulation of the NADPH oxidase RBOHD by the PRRassociated kinase BIK1 during plant immunity. Mol Cell 54:43-55.

Kang, L., Tang, X., and Mysore, K.S. (2004). Pseudomonas Type III effector AvrPto suppresses the programmed cell death induced by two nonhost pathogens in Nicotiana benthamiana and tomato. Mol Plant Microbe Interact 17:1328-1336.

Kanyuka, K., and Rudd, J.J. (2019). Cell surface immune receptors: the guardians of the plant's extracellular spaces. Curr Opin Plant Biol 50:1-8.

Kayes, J.M., and Clark, S.E. (1998). CLAVATA2, a regulator of meristem and organ development in Arabidopsis. Development 125:3843-3851.

Kim, T.W., Guan, S., Burlingame, A.L., and Wang, Z.Y. (2011). The CDG1 kinase mediates brassinosteroid signal transduction from BRI1 receptor kinase to BSU1 phosphatase and GSK3like kinase BIN2. Mol Cell 43:561-571.

Kim, Y.J., Lin, N.C., and Martin, G.B. (2002). Two distinct Pseudomonas effector proteins interact with the Pto kinase and activate plant immunity. Cell 109:589-598.

King, S.R., McLellan, H., Boevink, P.C., Armstrong, M.R., Bukharova, T., Sukarta, O., Win, J., Kamoun, S., Birch, P.R., and Banfield, M.J. (2014). Phytophthora infestans RXLR effector PexRD2 interacts with host MAPKKKE epsilon to suppress plant immune signaling. Plant Cell 26:1345-1359.

Kinoshita, A., Betsuyaku, S., Osakabe, Y., Mizuno, S., Nagawa, S., Stahl, Y., Simon, R., YamaguchiShinozaki, K., Fukuda, H., and Sawa, S. (2010). RPK2 is an essential receptor-like kinase that transmits the CLV3 signal in Arabidopsis. Development 137:3911-3920.

Kinoshita, A., Nakamura, Y., Sasaki, E., Kyozuka, J., Fukuda, H., and Sawa, S. (2007). Gain-of-function phenotypes of chemically synthetic CLAVATA3/ESR-related (CLE) peptides in Arabidopsis thaliana and Oryza sativa. Plant Cell Physiol 48:1821-1825.

Kishimoto, K., Kouzai, Y., Kaku, H., Shibuya, N., Minami, E., and Nishizawa, Y. (2010). Perception of the chitin oligosaccharides contributes to disease resistance to blast fungus Magnaporthe oryzae in rice. Plant J 64:343-354.

Kleinboelting, N., Huep, G., Kloetgen, A., Viehoever, P., and Weisshaar, B. (2012). GABI-Kat SimpleSearch: new features of the Arabidopsis thaliana T-DNA mutant database. Nucleic Acids Res 40:D1211-D1215.

Kondo, Y., Hirakawa, Y., Kieber, J.J., and Fukuda, H. (2011). CLE peptides can negatively regulate protoxylem vessel formation via cytokinin signaling. Plant Cell Physiol 52:37-48.

Kong, Q., Sun, T., Qu, N., Ma, J., Li, M., Cheng, Y.T., Zhang, Q., Wu, D., Zhang, Z., and Zhang, Y. (2016). Two redundant receptor-like cytoplasmic kinases function downstream of pattern recognition receptors to regulate activation of SA biosynthesis. Plant Physiol 171:1344-1354.

Kouzai, Y., Kaku, H., Shibuya, N., Minami, E., and Nishizawa, Y. (2013). Expression of the chimeric receptor between the chitin elicitor receptor CEBiP and the receptor-like protein kinase $\mathrm{Pi}-\mathrm{d} 2$ 
leads to enhanced responses to the chitin elicitor and disease resistance against Magnaporthe oryzae in rice. Plant Mol Biol 81:287-295.

Krol, E., Mentzel, T., Chinchilla, D., Boller, T., Felix, G., Kemmerling, B., Postel, S., Arents, M., Jeworutzki, E., Al-Rasheid, K.A., et al. (2010). Perception of the Arabidopsis danger signal peptide 1 involves the pattern recognition receptor AtPEPR1 and its close homologue AtPEPR2. J Biol Chem 285:13471-13479.

Kunze, G., Zipfel, C., Robatzek, S., Niehaus, K., Boller, T., and Felix, G. (2004). The N terminus of bacterial elongation factor Tu elicits innate immunity in Arabidopsis plants. Plant Cell 16:34963507.

Lacombe, S., Rougon-Cardoso, A., Sherwood, E., Peeters, N., Dahlbeck, D., van Esse, H.P., Smoker, M., Rallapalli, G., Thomma, B.P.H.J., Staskawicz, B., et al. (2010). Interfamily transfer of a plant pattern-recognition receptor confers broad-spectrum bacterial resistance. Nat Biotechnol 28:365369.

Laurent, F., Labesse, G., and de Wit, P. (2000). Molecular cloning and partial characterization of a plant VAP33 homologue with a major sperm protein domain. Biochem Biophys Res Commun 270:286292.

Laux, T., Mayer, K.F., Berger, J., and Jurgens, G. (1996). The WUSCHEL gene is required for shoot and floral meristem integrity in Arabidopsis. Development 122:87-96.

Lee, A.H., Middleton, M.A., Guttman, D.S., and Desveaux, D. (2013). Phytopathogen type III effectors as probes of biological systems. Microb Biotechnol 6:230-240.

Lee, J.S., Kuroha, T., Hnilova, M., Khatayevich, D., Kanaoka, M.M., McAbee, J.M., Sarikaya, M., Tamerler, C., and Torii, K.U. (2012). Direct interaction of ligand-receptor pairs specifying stomatal patterning. Genes Dev 26:126-136.

Leslie, M.E., Lewis, M.W., Youn, J.Y., Daniels, M.J., and Liljegren, S.J. (2010). The EVERSHED receptor-like kinase modulates floral organ shedding in Arabidopsis. Development 137:467-476.

Li, B., Lu, D., and Shan, L. (2014a). Ubiquitination of pattern recognition receptors in plant innate immunity. Mol Plant Pathol 15:737-746.

Li, C., Wu, H.M., and Cheung, A.Y. (2016a). FERONIA and her pals: Functions and mechanisms. Plant Physiol 171:2379-2392.

Li, J., and Chory, J. (1997). A putative leucine-rich repeat receptor kinase involved in brassinosteroid signal transduction. Cell 90:929-938.

Li, J., Wen, J., Lease, K.A., Doke, J.T., Tax, F.E., and Walker, J.C. (2002). BAK1, an Arabidopsis LRR receptor-like protein kinase, interacts with BRI1 and modulates brassinosteroid signaling. Cell 110:213-222.

Li, L., Kim, P., Yu, L., Cai, G., Chen, S., Alfano, J.R., and Zhou, J.-M. (2016b). Activation-dependent destruction of a co-receptor by a Pseudomonas syringae effector dampens plant immunity. Cell Host Microbe 20:504-514.

Li, L., Li, M., Yu, L., Zhou, Z., Liang, X., Liu, Z., Cai, G., Gao, L., Zhang, X., Wang, Y., et al. (2014b). The FLS2-associated kinase BIK1 directly phosphorylates the NADPH oxidase RbohD to control plant immunity. Cell Host Microbe 15:329-338.

Li, L., Yu, Y., Zhou, Z., and Zhou, J.-M. (2016c). Plant pattern-recognition receptors controlling innate immunity. Sci China Life Sci 59:878-888.

Li, S., Liu, Y., Zheng, L., Chen, L., Li, N., Corke, F., Lu, Y., Fu, X., Zhu, Z., Bevan, M.W., et al. (2012). The plant-specific G protein y subunit AGG3 influences organ size and shape in Arabidopsis thaliana. New Phytol 194:690-703.

Liang, X., Ding, P., Lian, K., Wang, J., Ma, M., Li, L., Li, L., Li, M., Zhang, X., Chen, S., et al. (2016). Arabidopsis heterotrimeric $\mathrm{G}$ proteins regulate immunity by directly coupling to the FLS2 receptor. eLife 5:e13568.

Liang, X., Ma, M., Zhou, Z., Wang, J., Yang, X., Rao, S., Bi, G., Li, L., Zhang, X., Chai, J., et al. (2018). Ligand-triggered de-repression of Arabidopsis heterotrimeric $\mathrm{G}$ proteins coupled to immune receptor kinases. Cell Res 28:529-543.

Liang, X., and Zhou, J.-M. (2018). Receptor-like cytoplasmic kinases: central players in plant receptor kinase-mediated signaling. Annu Rev Plant Biol 69:267-299.

Liebrand, T.W.H., Smit, P., Abd-El-Haliem, A.M., de Jonge, R., Cordewener, J.H.G., America, A.H.P., Sklenar, J., Jones, A.M.E., Robatzek, S., Thomma, B.P.H.J., et al. (2012). Endoplasmic reticulumquality control chaperones facilitate the biogenesis of Cf receptor-like proteins involved in pathogen resistance of tomato. Plant Physiol 159:1819-1833.

Liebrand, T.W.H., van den Berg, G.C.M., Zhang, Z., Smit, P., Cordewener, J.H.G., America, A.H.P., Sklenar, J., Jones, A.M.E., Tameling, W.I.L., Robatzek, S., et al. (2013). Receptor-like kinase SOBIR1/EVR interacts with receptor-like proteins in plant immunity against fungal infection. Proc 


\section{References}

Natl Acad Sci USA 110:10010-10015.

Liebrand, T.W.H., van den Burg, H.A., and Joosten, M.H.A.J. (2014). Two for all: receptor-associated kinases SOBIR1 and BAK1. Trends Plant Sci 19:123-132.

Liljegren, S.J. (2012). Organ abscission: exit strategies require signals and moving traffic. Curr Opin Plant Biol 15:670-676.

Lin, G., Zhang, L., Han, Z., Yang, X., Liu, W., Li, E., Chang, J., Qi, Y., Shpak, E.D., and Chai, J. (2017). A receptor-like protein acts as a specificity switch for the regulation of stomatal development. Genes Dev 31:927-938.

Lin, W., Li, B., Lu, D., Chen, S., Zhu, N., He, P., and Shan, L. (2014). Tyrosine phosphorylation of protein kinase complex BAK1/BIK1 mediates Arabidopsis innate immunity. Proc Natl Acad Sci USA 111:3632-3637.

Lin, W., Lu, D., Gao, X., Jiang, S., Ma, X., Wang, Z., Mengiste, T., He, P., and Shan, L. (2013a). Inverse modulation of plant immune and brassinosteroid signaling pathways by the receptor-like cytoplasmic kinase BIK1. Proc Natl Acad Sci USA 110:12114-12119.

Lin, W., Ma, X., Shan, L., and He, P. (2013b). Big roles of small kinases: the complex functions of receptor-like cytoplasmic kinases in plant immunity and development. J Integr Plant Biol 55:11881197.

Lin, Z.J., Liebrand, T.W., Yadeta, K.A., and Coaker, G. (2015). PBL13 is a serine/threonine protein kinase that negatively regulates Arabidopsis immune responses. Plant Physiol 169:2950-2962.

Liu, Y., Huang, X., Li, M., He, P., and Zhang, Y. (2016). Loss-of-function of Arabidopsis receptor-like kinase BIR1 activates cell death and defense responses mediated by BAK1 and SOBIR1. New Phytol 212:637-645.

Loh, Y.T., and Martin, G.B. (1995). The Pto bacterial resistance gene and the Fen insecticide sensitivity gene encode functional protein kinases with serine/threonine specificity. Plant Physiol 108:17351739.

Lu, D., Lin, W., Gao, X., Wu, S., Cheng, C., Avila, J., Heese, A., Devarenne, T.P., He, P., and Shan, L. (2011). Direct ubiquitination of pattern recognition receptor FLS2 attenuates plant innate immunity. Science 332:1439-1442.

Lu, D., Wu, S., Gao, X., Zhang, Y., Shan, L., and He, P. (2010). A receptor-like cytoplasmic kinase, BIK1, associates with a flagellin receptor complex to initiate plant innate immunity. Proc Natl Acad Sci USA 107:496-501.

Luderer, R., Rivas, S., Nürnberger, T., Mattei, B., Van den Hooven, H.W., Van der Hoorn, R.A., Romeis, T., Wehrfritz, J.-M., Blume, B., and Nennstiel, D. (2001). No evidence for binding between resistance gene product Cf-9 of tomato and avirulence gene product Avr9 of Cladosporium fulvum. Mol Plant Microbe Interact 14:867-876.

Luderer, R., Takken, F.L., de Wit, P.J., and Joosten, M.H. (2002). Cladosporium fulvum overcomes Cf2-mediated resistance by producing truncated Avr2 elicitor proteins. Mol Microbiol 45:875-884.

Ma, C., Liu, Y., Bai, B., Han, Z., Tang, J., Zhang, H., Yaghmaiean, H., Zhang, Y., and Chai, J. (2017). Structural basis for BIR1-mediated negative regulation of plant immunity. Cell Res 27:1521-1524.

Ma, L., and Borhan, M.H. (2015). The receptor-like kinase SOBIR1 interacts with Brassica napus LepR3 and is required for Leptosphaeria maculans AvrLm1-triggered immunity. Front Plant Sci 6:933.

Ma, X., Xu, G., He, P., and Shan, L. (2016). SERKing coreceptors for receptors. Trends Plant Sci 21:1017-1033.

Ma, Y., Zhao, Y., Walker, R.K., and Berkowitz, G.A. (2013). Molecular steps in the immune signaling pathway evoked by plant elicitor peptides: $\mathrm{Ca}^{2+}$-dependent protein kinases, nitric oxide, and reactive oxygen species are downstream from the early $\mathrm{Ca}^{2+}$ signal. Plant Physiol 163:1459-1471.

Ma, Z., Song, T., Zhu, L., Ye, W., Wang, Y., Shao, Y., Dong, S., Zhang, Z., Dou, D., Zheng, X., et al. (2015). A Phytophthora sojae glycoside hydrolase 12 protein is a major virulence factor during soybean infection and is recognized as a PAMP. Plant Cell 27:2057-2072.

Macho, A.P., Schwessinger, B., Ntoukakis, V., Brutus, A., Segonzac, C., Roy, S., Kadota, Y., Oh, M.H., Sklenar, J., Derbyshire, P., et al. (2014). A bacterial tyrosine phosphatase inhibits plant pattern recognition receptor activation. Science 343:1509-1512.

Macho, A.P., and Zipfel, C. (2015). Targeting of plant pattern recognition receptor-triggered immunity by bacterial type-III secretion system effectors. Curr Opin Microbiol 23:14-22.

Maldonado-Bonilla, L.D., Eschen-Lippold, L., Gago-Zachert, S., Tabassum, N., Bauer, N., Scheel, D., and Lee, J. (2013). The Arabidopsis tandem zinc finger 9 protein binds RNA and mediates pathogen-associated molecular pattern-triggered immune responses. Plant Cell Physiol 55:412425.

Manfredini, C., Sicilia, F., Ferrari, S., Pontiggia, D., Salvi, G., Caprari, C., Lorito, M., and De Lorenzo, G. (2005). Polygalacturonase-inhibiting protein 2 of Phaseolus vulgaris inhibits BcPG1, a 
polygalacturonase of Botrytis cinerea important for pathogenicity, and protects transgenic plants from infection. Physiol Mol Plant Path 67:108-115.

Mang, H., Feng, B., Hu, Z., Boisson-Dernier, A., Franck, C.M., Meng, X., Huang, Y., Zhou, J., Xu, G., Wang, T., et al. (2017). Differential regulation of two-tiered plant immunity and sexual reproduction by ANXUR receptor-like kinases. Plant Cell 29:3140-3156.

Martin, G.B., Brommonschenkel, S.H., Chunwongse, J., Frary, A., Ganal, M.W., Spivey, R., Wu, T., Earle, E.D., and Tanksley, S.D. (1993). Map-based cloning of a protein kinase gene conferring disease resistance in tomato. Science 262:1432-1436.

Matsubayashi, Y. (2014). Posttranslationally modified small-peptide signals in plants. Annu Rev Plant Biol 65:385-413.

Matsubayashi, Y., Ogawa, M., Kihara, H., Niwa, M., and Sakagami, Y. (2006). Disruption and overexpression of Arabidopsis phytosulfokine receptor gene affects cellular longevity and potential for growth. Plant Physiol 142:45-53.

Matsubayashi, Y., Ogawa, M., Morita, A., and Sakagami, Y. (2002). An LRR receptor kinase involved in perception of a peptide plant hormone, phytosulfokine. Science 296:1470-1472.

Mattei, B., Spinelli, F., Pontiggia, D., and De Lorenzo, G. (2016). Comprehensive analysis of the membrane phosphoproteome regulated by oligogalacturonides in Arabidopsis thaliana. Front Plant Sci 7:1107.

Melech-Bonfil, S., and Sessa, G. (2010). Tomato MAPKKKE is a positive regulator of cell-death signaling networks associated with plant immunity. Plant J 64:379-391.

Mendes, B., Cardoso, S., Boscariol - Camargo, R., Cruz, R., Mourão Filho, F., and Bergamin Filho, A. (2010). Reduction in susceptibility to Xanthomonas axonopodis pv. citri in transgenic Citrus sinensis expressing the rice Xa21 gene. Plant Physiol 59:68-75.

Meng, X., Chen, X., Mang, H., Liu, C., Yu, X., Gao, X., Torii, K.U., He, P., and Shan, L. (2015). Differential function of Arabidopsis SERK family receptor-like kinases in stomatal patterning. Curr Biol 25:2361-2372.

Meng, X., and Zhang, S. (2013). MAPK cascades in plant disease resistance signaling. Annu Rev Phytopathol 51:245-266.

Meng, X., Zhou, J., Tang, J., Li, B., de Oliveira, M.V.V., Chai, J., He, P., and Shan, L. (2016). Ligandinduced receptor-like kinase complex regulates floral organ abscission in Arabidopsis. Cell Rep 14:1330-1338.

Mesarich, C.H., Stergiopoulos, I., Beenen, H.G., Cordovez, V., Guo, Y., Karimi Jashni, M., Bradshaw, R.E., and de Wit, P.J. (2016). A conserved proline residue in Dothideomycete Avr4 effector proteins is required to trigger a Cf-4-dependent hypersensitive response. Mol Plant Pathol 17:8495.

Milhinhos, A., Vera-Sirera, F., Blanco-Tourinan, N., Mari-Carmona, C., Carrio-Segui, A., Forment, J., Champion, C., Thamm, A., Urbez, C., Prescott, H., et al. (2019). SOBIR1/EVR prevents precocious initiation of fiber differentiation during wood development through a mechanism involving BP and ERECTA. Proc Natl Acad Sci USA 116:18710-18716.

Mithoe, S.C., Ludwig, C., Pel, M.J., Cucinotta, M., Casartelli, A., Mbengue, M., Sklenar, J., Derbyshire, P., Robatzek, S., Pieterse, C.M., et al. (2016). Attenuation of pattern recognition receptor signaling is mediated by a MAP kinase kinase kinase. EMBO Rep 17:441-454.

Mithoe, S.C., and Menke, F.L. (2018). Regulation of pattern recognition receptor signalling by phosphorylation and ubiquitination. Curr Opin Plant Biol 45:162-170.

Miwa, H., Betsuyaku, S., Iwamoto, K., Kinoshita, A., Fukuda, H., and Sawa, S. (2008). The receptor-like kinase SOL2 mediates CLE signaling in Arabidopsis. Plant Cell Physiol 49:1752-1757.

Miyazaki, S., Murata, T., Sakurai-Ozato, N., Kubo, M., Demura, T., Fukuda, H., and Hasebe, M. (2009). ANXUR1 and 2, sister genes to FERONIA/SIRENE, are male factors for coordinated fertilization. Curr Biol 19:1327-1331.

Monaghan, J., Matschi, S., Shorinola, O., Rovenich, H., Matei, A., Segonzac, C., Malinovsky, F.G., Rathjen, J.P., MacLean, D., Romeis, T., et al. (2014). The calcium-dependent protein kinase CPK28 buffers plant immunity and regulates BIK1 turnover. Cell Host Microbe 16:605-615.

Monaghan, J., and Zipfel, C. (2012). Plant pattern recognition receptor complexes at the plasma membrane. Curr Opin Plant Biol 15:349-357.

Mucyn, T.S., Clemente, A., Andriotis, V.M., Balmuth, A.L., Oldroyd, G.E., Staskawicz, B.J., and Rathjen, J.P. (2006). The tomato NBARC-LRR protein Prf interacts with Pto kinase in vivo to regulate specific plant immunity. Plant Cell 18:2792-2806.

Müller, R., Bleckmann, A., and Simon, R. (2008). The receptor kinase CORYNE of Arabidopsis transmits the stem cell-limiting signal CLAVATA3 independently of CLAVATA1. Plant Cell 20:934-946.

Nadeau, J.A., and Sack, F.D. (2002). Control of stomatal distribution on the Arabidopsis leaf surface. 


\section{References}

Science 296:1697-1700.

Nam, K.H., and Li, J. (2002). BRI1/BAK1, a receptor kinase pair mediating brassinosteroid signaling. Cell 110:203-212.

Nekrasov, V., Ludwig, A.A., and Jones, J.D. (2006). CITRX thioredoxin is a putative adaptor protein connecting Cf-9 and the ACIK1 protein kinase during the Cf-9/Avr9- induced defence response. FEBS Lett 580:4236-4241.

Nicaise, V., Joe, A., Jeong, B.R., Korneli, C., Boutrot, F., Westedt, I., Staiger, D., Alfano, J.R., and Zipfel, C. (2013). Pseudomonas HopU1 modulates plant immune receptor levels by blocking the interaction of their mRNAs with GRP7. EMBO J 32:701-712.

Nimchuk, Z.L., Tarr, P.T., and Meyerowitz, E.M. (2011). An evolutionarily conserved pseudokinase mediates stem cell production in plants. Plant Cell 23:851-854.

Ogawa, M., Shinohara, H., Sakagami, Y., and Matsubayashi, Y. (2008). Arabidopsis CLV3 peptide directly binds CLV1 ectodomain. Science 319:294.

Oome, S., Raaymakers, T.M., Cabral, A., Samwel, S., Böhm, H., Albert, I., Nürnberger, T., and van den Ackerveken, G.F.J.M. (2014). Nep1-like proteins from three kingdoms of life act as a microbeassociated molecular pattern in Arabidopsis. Proc Natl Acad Sci USA 111:16955-16960.

Orosa, B., He, Q., Mesmar, J., Gilroy, E.M., McLellan, H., Yang, C., Craig, A., Bailey, M., Zhang, C., Moore, J.D., et al. (2017). BTB-BACK domain protein POB1 suppresses immune cell death by targeting ubiquitin E3 ligase PUB17 for degradation. PLoS Genet 13:e1006540.

Ott, T. (2017). Membrane nanodomains and microdomains in plant-microbe interactions. Curr Opin Plant Biol 40:82-88

Pan, L., Lv, S., Yang, N., Lv, Y., Liu, Z., Wu, J., and Wang, G. (2016). The multifunction of CLAVATA2 in plant development and immunity. Front Plant Sci 7:1573.

Pandey, S. (2019). Heterotrimeric G-protein signaling in plants: Conserved and novel mechanisms. Annu Rev Plant Biol 70:213-238.

Park, C.J., Peng, Y., Chen, X., Dardick, C., Ruan, D., Bart, R., Canlas, P.E., and Ronald, P.C. (2008). Rice $X B 15$, a protein phosphatase $2 C$, negatively regulates cell death and $X A 21$-mediated innate immunity. PLoS Biol 6:e231.

Patharkar, O.R., and Walker, J.C. (2018). Advances in abscission signaling. J Exp Bot 69:733-740.

Pecenkova, T., Hala, M., Kulich, I., Kocourkova, D., Drdova, E., Fendrych, M., Toupalova, H., and Zarsky, V. (2011). The role for the exocyst complex subunits Exo70B2 and Exo70H1 in the plant-pathogen interaction. J Exp Bot 62:2107-2116.

Pecher, P., Eschen-Lippold, L., Herklotz, S., Kuhle, K., Naumann, K., Bethke, G., Uhrig, J., Weyhe, M., Scheel, D., and Lee, J. (2014). The Arabidopsis thaliana mitogen-activated protein kinases MPK3 and MPK6 target a subclass of 'VQ-motif'-containing proteins to regulate immune responses. New Phytol 203:592-606.

Pedley, K.F., and Martin, G.B. (2003). Molecular basis of Pto-mediated resistance to bacterial speck disease in tomato. Annu Rev Phytopathol 41:215-243.

Peng, K.C., Wang, C.W., Wu, C.H., Huang, C.T., and Liou, R.F. (2015). Tomato SOBIR1/EVR homologs are involved in elicitin perception and plant defense against the oomycetepathogen Phytophthora parasitica. Mol Plant Microbe Interact 28:913-926.

Perraki, A., DeFalco, T.A., Derbyshire, P., Avila, J., Sere, D., Sklenar, J., Qi, X., Stransfeld, L., Schwessinger, B., Kadota, Y., et al. (2018). Phosphocode-dependent functional dichotomy of a common co-receptor in plant signalling. Nature 561:248-252.

Postma, J., Liebrand, T.W.H., Bi, G., Evrard, A., Bye, R.R., Mbengue, M., Kuhn, H., Joosten, M.H.A.J., and Robatzek, S. (2016). Avr4 promotes Cf-4 receptor-like protein association with the BAK1/SERK3 receptor-like kinase to initiate receptor endocytosis and plant immunity. New Phytol 210:627-642.

Pruitt, R.N., Schwessinger, B., Joe, A., Thomas, N., Liu, F., Albert, M., Robinson, M.R., Chan, L.J.G., Luu, D.D., Chen, H., et al. (2015). The rice immune receptor XA21 recognizes a tyrosine-sulfated protein from a Gram-negative bacterium. Sci Adv 1:e1500245.

Ranf, S. (2017). Sensing of molecular patterns through cell surface immune receptors. Curr Opin Plant Biol 38:68-77.

Ranf, S., Eschen-Lippold, L., Frohlich, K., Westphal, L., Scheel, D., and Lee, J. (2014). Microbeassociated molecular pattern-induced calcium signaling requires the receptor-like cytoplasmic kinases, PBL1 and BIK1. BMC Plant Biol 14:374.

Rao, S., Zhou, Z., Miao, P., Bi, G., Hu, M., Wu, Y., Feng, F., Zhang, X., and Zhou, J.-M. (2018). Roles of receptor-like cytoplasmic kinase VII members in pattern-triggered immune signaling. Plant Physiol 177:1679-1690.

Ren, S.C., Song, X.F., Chen, W.Q., Lu, R., Lucas, W.J., and Liu, C.M. (2019). CLE25 peptide regulates 
phloem initiation in Arabidopsis through a CLERK-CLV2 receptor complex. J Integr Plant Biol 61:1043-1061.

Rivas, S., Rougon-Cardoso, A., Smoker, M., Schauser, L., Yoshioka, H., and Jones, J.D. (2004). CITRX thioredoxin interacts with the tomato $\mathrm{Cf}-9$ resistance protein and negatively regulates defence. EMBO J 23:2156-2165.

Robatzek, S., Bittel, P., Chinchilla, D., Kochner, P., Felix, G., Shiu, S.H., and Boller, T. (2007). Molecular identification and characterization of the tomato flagellin receptor LeFLS2, an orthologue of Arabidopsis FLS2 exhibiting characteristically different perception specificities. Plant Mol Biol 64:539-547.

Romeis, T., Ludwig, A.A., Martin, R., and Jones, J.D. (2001). Calcium-dependent protein kinases play an essential role in a plant defence response. EMBO J 20:5556-5567.

Ron, M., and Avni, A. (2004). The receptor for the fungal elicitor ethylene-inducing xylanase is a member of a resistance-like gene family in tomato. Plant Cell 16:1604-1615.

Rotman, N., Rozier, F., Boavida, L., Dumas, C., Berger, F., and Faure, J.E. (2003). Female control of male gamete delivery during fertilization in Arabidopsis thaliana. Curr Biol 13:432-436.

Roux, M., Schwessinger, B., Albrecht, C., Chinchilla, D., Jones, A., Holton, N., Malinovsky, F.G., Tor, M., de Vries, S., and Zipfel, C. (2011). The Arabidopsis leucine-rich repeat receptor-like kinases BAK1/SERK3 and BKK1/SERK4 are required for innate immunity to hemibiotrophic and biotrophic pathogens. Plant Cell 23:2440-2455.

Rowland, O., Ludwig, A.A., Merrick, C.J., Baillieul, F., Tracy, F.E., Durrant, W.E., Fritz-Laylin, L., Nekrasov, V., Sjolander, K., Yoshioka, H., et al. (2005). Functional analysis of Avr9/Cf-9 rapidly elicited genes identifies a protein kinase, ACIK1, that is essential for full Cf-9-dependent disease resistance in tomato. Plant Cell 17:295-310.

Ruijter, J.M., Ramakers, C., Hoogaars, W.M., Karlen, Y., Bakker, O., van den Hoff, M.J., and Moorman, A.F. (2009). Amplification efficiency: linking baseline and bias in the analysis of quantitative PCR data. Nucleic Acids Res 37:e45.

Saand, M.A., Xu, Y.P., Li, W., Wang, J.P., and Cai, X.Z. (2015). Cyclic nucleotide gated channel gene family in tomato: genome-wide identification and functional analyses in disease resistance. Front Plant Sci 6:303.

Saijo, Y., Loo, E.P., and Yasuda, S. (2018). Pattern recognition receptors and signaling in plant-microbe interactions. Plant J 93:592-613.

Salmeron, J.M., Barker, S.J., Carland, F.M., Mehta, A.Y., and Staskawicz, B.J. (1994). Tomato mutants altered in bacterial disease resistance provide evidence for a new locus controlling pathogen recognition. Plant Cell 6:511-520.

Santiago, J., Brandt, B., Wildhagen, M., Hohmann, U., Hothorn, L.A., Butenko, M.A., and Hothorn, M. (2016). Mechanistic insight into a peptide hormone signaling complex mediating floral organ abscission. eLife 5:e15075.

Santiago, J., Henzler, C., and Hothorn, M. (2013). Molecular mechanism for plant steroid receptor activation by somatic embryogenesis co-receptor kinases. Science 341:889-892.

Schoof, H., Lenhard, M., Haecker, A., Mayer, K.F., Jürgens, G., and Laux, T. (2000). The stem cell population of Arabidopsis shoot meristems is maintained by a regulatory loop between the CLAVATA and WUSCHEL genes. Cell 100:635-644.

Schoonbeek, H.J., Wang, H.H., Stefanato, F.L., Craze, M., Bowden, S., Wallington, E., Zipfel, C., and Ridout, C.J. (2015). Arabidopsis EF-Tu receptor enhances bacterial disease resistance in transgenic wheat. New Phytol 206:606-613.

Schulze, B., Mentzel, T., Jehle, A.K., Mueller, K., Beeler, S., Boller, T., Felix, G., and Chinchilla, D. (2010). Rapid heteromerization and phosphorylation of ligand-activated plant transmembrane receptors and their associated kinase BAK1. J Biol Chem 285:9444-9451.

Schweighofer, A., Kazanaviciute, V., Scheikl, E., Teige, M., Doczi, R., Hirt, H., Schwanninger, M., Kant, M., Schuurink, R., Mauch, F., et al. (2007). The PP2C-type phosphatase AP2C1, which negatively regulates MPK4 and MPK6, modulates innate immunity, jasmonic acid, and ethylene levels in Arabidopsis. Plant Cell 19:2213-2224.

Schwessinger, B., Bahar, O., Thomas, N., Holton, N., Nekrasov, V., Ruan, D., Canlas, P.E., Daudi, A., Petzold, C.J., Singan, V.R., et al. (2015). Transgenic expression of the dicotyledonous pattern recognition receptor EFR in rice leads to ligand-dependent activation of defense responses. PLoS Pathog 11:e1004809.

Schwessinger, B., Roux, M., Kadota, Y., Ntoukakis, V., Sklenar, J., Jones, A., and Zipfel, C. (2011). Phosphorylation-dependent differential regulation of plant growth, cell death, and innate immunity by the regulatory receptor-like kinase BAK1. PLoS Genet 7:e1002046.

Schwizer, S., Kraus, C.M., Dunham, D.M., Zheng, Y., Fernandez-Pozo, N., Pombo, M.A., Fei, Z., 


\section{References}

Chakravarthy, S., and Martin, G.B. (2017). The tomato kinase Pti1 contributes to production of reactive oxygen species in response to two flagellin-derived peptides and promotes resistance to Pseudomonas syringae infection. Mol Plant Microbe Interact 30:725-738.

Scofield, S.R., Tobias, C.M., Rathjen, J.P., Chang, J.H., Lavelle, D.T., Michelmore, R.W., and Staskawicz, B.J. (1996). Molecular basis of gene-for-gene specificity in bacterial speck disease of tomato. Science 274:2063-2065.

Segonzac, C., Macho, A.P., Sanmartin, M., Ntoukakis, V., Sanchez-Serrano, J.J., and Zipfel, C. (2014). Negative control of BAK1 by protein phosphatase 2A during plant innate immunity. EMBO $\mathrm{J}$ 33:2069-2079.

Shan, L., He, P., Li, J., Heese, A., Peck, S.C., Nurnberger, T., Martin, G.B., and Sheen, J. (2008). Bacterial effectors target the common signaling partner BAK1 to disrupt multiple MAMP receptorsignaling complexes and impede plant immunity. Cell Host Microbe 4:17-27.

Shan, L., Thara, V.K., Martin, G.B., Zhou, J.M., and Tang, X. (2000). The Pseudomonas AvrPto protein is differentially recognized by tomato and tobacco and is localized to the plant plasma membrane. Plant Cell 12:2323-2338.

Shao, F., Golstein, C., Ade, J., Stoutemyer, M., Dixon, J.E., and Innes, R.W. (2003). Cleavage of Arabidopsis PBS1 by a bacterial type III effector. Science 301:1230-1233.

She, J., Han, Z., Kim, T.W., Wang, J., Cheng, W., Chang, J., Shi, S., Wang, J., Yang, M., Wang, Z.Y., et al. (2011). Structural insight into brassinosteroid perception by BRI1. Nature 474:472-476.

Shen, Q., Bourdais, G., Pan, H., Robatzek, S., and Tang, D. (2017). Arabidopsis glycosylphosphatidylinositol-anchored protein LLG1 associates with and modulates FLS2 to regulate innate immunity. Proc Natl Acad Sci USA 114:5749-5754.

Shi, H., Shen, Q., Qi, Y., Yan, H., Nie, H., Chen, Y., Zhao, T., Katagiri, F., and Tang, D. (2013). BRSIGNALING KINASE1 physically associates with FLAGELLIN SENSING2 and regulates plant innate immunity in Arabidopsis. Plant Cell 25:1143-1157.

Shinohara, H., and Matsubayashi, Y. (2015). Reevaluation of the CLV3-receptor interaction in the shoot apical meristem: dissection of the CLV3 signaling pathway from a direct ligand-binding point of view. Plant J 82:328-336.

Shiu, S.H., Karlowski, W.M., Pan, R., Tzeng, Y.H., Mayer, K.F., and Li, W.H. (2004). Comparative analysis of the receptor-like kinase family in Arabidopsis and rice. Plant Cell 16:1220-1234.

Shpak, E.D., McAbee, J.M., Pillitteri, L.J., and Torii, K.U. (2005). Stomatal patterning and differentiation by synergistic interactions of receptor kinases. Science 309:290-293.

Shubchynskyy, V., Boniecka, J., Schweighofer, A., Simulis, J., Kvederaviciute, K., Stumpe, M., Mauch, F., Balazadeh, S., Mueller-Roeber, B., Boutrot, F., et al. (2017). Protein phosphatase AP2C1 negatively regulates basal resistance and defense responses to Pseudomonas syringae. J Exp Bot 68:1169-1183.

Smakowska-Luzan, E., Mott, G.A., Parys, K., Stegmann, M., Howton, T.C., Layeghifard, M., Neuhold, J., Lehner, A., Kong, J., Grunwald, K., et al. (2018). An extracellular network of Arabidopsis leucine-rich repeat receptor kinases. Nature 553:342-346.

Somssich, M., Bleckmann, A., and Simon, R. (2016a). Shared and distinct functions of the pseudokinase CORYNE (CRN) in shoot and root stem cell maintenance of Arabidopsis. J Exp Bot 67:4901-4915.

Somssich, M., Je, B.I., Simon, R., and Jackson, D. (2016b). CLAVATA-WUSCHEL signaling in the shoot meristem. Development 143:3238-3248.

Somssich, M., Ma, Q., Weidtkamp-Peters, S., Stahl, Y., Felekyan, S., Bleckmann, A., Seidel, C.A., and Simon, R. (2015). Real-time dynamics of peptide ligand-dependent receptor complex formation in planta. Sci Signal 8:ra76.

Song, S.K., Lee, M.M., and Clark, S.E. (2006). POL and PLL1 phosphatases are CLAVATA1 signaling intermediates required for Arabidopsis shoot and floral stem cells. Development 133:4691-4698.

Song, W.-Y., Wang, G.-L., Chen, L.-L., Kim, H.-S., Pi, L.-Y., Holsten, T., Gardner, J., Wang, B., Zhai, W.X., Zhu, L.-H., et al. (1995). A receptor kinase-like protein encoded by the rice disease resistance gene, Xa21. Science 270:1804-1806.

Song, W., Han, Z., Wang, J., Lin, G., and Chai, J. (2017a). Structural insights into ligand recognition and activation of plant receptor kinases. Curr Opin Struct Biol 43:18-27.

Song, Y., Liu, L., Wang, Y., Valkenburg, D.J., Zhang, X., Zhu, L., and Thomma, B.P.H.J. (2017b). Transfer of tomato immune receptor Ve1 confers Ave1-dependent Verticillium resistance in tobacco and cotton. Plant Biotechnol J 16:638-648.

Srivastava, R., Liu, J.X., Guo, H., Yin, Y., and Howell, S.H. (2009). Regulation and processing of a plant peptide hormone, AtRALF23, in Arabidopsis. Plant J 59:930-939.

Stegmann, M., Anderson, R.G., Ichimura, K., Pecenkova, T., Reuter, P., Zarsky, V., McDowell, J.M., Shirasu, K., and Trujillo, M. (2012). The ubiquitin ligase PUB22 targets a subunit of the exocyst 
complex required for PAMP-triggered responses in Arabidopsis. Plant Cell 24:4703-4716.

Stegmann, M., Monaghan, J., Smakowska-Luzan, E., Rovenich, H., Lehner, A., Holton, N., Belkhadir, Y., and Zipfel, C. (2017). The receptor kinase FER is a RALF-regulated scaffold controlling plant immune signaling. Science 355:287-289.

Stergiopoulos, I., and de Wit, P.J.G.M. (2009). Fungal effector proteins. Annu Rev Phytopathol 47:233263.

Stone, J.M., Trotochaud, A.E., Walker, J.C., and Clark, S.E. (1998). Control of meristem development by CLAVATA1 receptor kinase and kinase-associated protein phosphatase interactions. Plant Physiol 117:1217-1225.

Stulemeijer, I.J.E., Stratmann, J.W., and Joosten, M.H.A.J. (2007). Tomato mitogen-activated protein kinases LeMPK1, LeMPK2, and LeMPK3 are activated during the Cf-4/Avr4-induced hypersensitive response and have distinct phosphorylation specificities. Plant Physiol 144:14811494.

Sun, T., Nitta, Y., Zhang, Q., Wu, D., Tian, H., Lee, J.S., and Zhang, Y. (2018). Antagonistic interactions between two MAP kinase cascades in plant development and immune signaling. EMBO Rep 19.

Sun, Y., Han, Z., Tang, J., Hu, Z., Chai, C., Zhou, B., and Chai, J. (2013a). Structure reveals that BAK1 as a co-receptor recognizes the BRI1-bound brassinolide. Cell Res 23:1326-1329.

Sun, Y., Li, L., Macho, A.P., Han, Z., Hu, Z., Zipfel, C., Zhou, J.-M., and Chai, J. (2013b). Structural basis for flg22-induced activation of the Arabidopsis FLS2-BAK1 immune complex. Science 342:624628.

Suzaki, T., Sato, M., Ashikari, M., Miyoshi, M., Nagato, Y., and Hirano, H.Y. (2004). The gene FLORAL ORGAN NUMBER1 regulates floral meristem size in rice and encodes a leucine-rich repeat receptor kinase orthologous to Arabidopsis CLAVATA1. Development 131:5649-5657.

Swiderski, M.R., and Innes, R.W. (2001). The Arabidopsis PBS1 resistance gene encodes a member of a novel protein kinase subfamily. Plant J 26:101-112.

Taguchi-Shiobara, F., Yuan, Z., Hake, S., and Jackson, D. (2001). The fasciated ear2 gene encodes a leucine-rich repeat receptor-like protein that regulates shoot meristem proliferation in maize. Genes Dev 15:2755-2766.

Takahashi, T., Shibuya, H., and Ishikawa, A. (2016). SOBIR1 contributes to non-host resistance to Magnaporthe oryzae in Arabidopsis. Biosci Biotechnol Biochem 80:1577-1579.

Takai, R., Isogai, A., Takayama, S., and Che, F.S. (2008). Analysis of flagellin perception mediated by flg22 receptor OsFLS2 in rice. Mol Plant Microbe Interact 21:1635-1642.

Takken, F.L., Thomas, C.M., Joosten, M.H., Golstein, C., Westerink, N., Hille, J., Nijkamp, H.J., de Wit, P.J., and Jones, J.D. (1999). A second gene at the tomato Cf-4 locus confers resistance to Cladosporium fulvum through recognition of a novel avirulence determinant. Plant J 20:279-288.

Tang, D., Wang, G., and Zhou, J.M. (2017). Receptor kinases in plant-pathogen interactions: more than pattern recognition. Plant Cell 29:618-637.

Tang, J., Han, Z., Sun, Y., Zhang, H., Gong, X., and Chai, J. (2015). Structural basis for recognition of an endogenous peptide by the plant receptor kinase PEPR1. Cell Res 25:110-120.

Tang, R.J., and Luan, S. (2017). Regulation of calcium and magnesium homeostasis in plants: from transporters to signaling network. Curr Opin Plant Biol 39:97-105.

Tang, W., Kim, T.W., Oses-Prieto, J.A., Sun, Y., Deng, Z., Zhu, S., Wang, R., Burlingame, A.L., and Wang, Z.Y. (2008). BSKs mediate signal transduction from the receptor kinase BRI1 in Arabidopsis. Science 321:557-560.

Thomas, C.M., Jones, D.A., Parniske, M., Harrison, K., Balint-Kurti, P.J., Hatzixanthis, K., and Jones, J.D.G. (1997). Characterization of the tomato Cf-4 gene for resistance to Cladosporium fulvum identifies sequences that determine recognitional specificity in Cf-4 and Cf-9. Plant Cell 9:22092224.

Thomas, C.M., Tang, S., Hammond-Kosack, K., and Jones, J.D. (2000). Comparison of the hypersensitive response induced by the tomato $\mathrm{Cf}-4$ and $\mathrm{Cf}-9$ genes in Nicotiana spp. Mol Plant Microbe Interact 13:465-469.

Thomas, N.C., Oksenberg, N., Liu, F., Caddell, D., Nalyvayko, A., Nguyen, Y., Schwessinger, B., and Ronald, P.C. (2017). The rice XA21 ectodomain fused to the Arabidopsis EFR cytoplasmic domain confers resistance to Xanthomonas oryzae pv. oryzae. PeerJ 6:e4456.

Thomma, B.P., Nurnberger, T., and Joosten, M.H. (2011). Of PAMPs and effectors: the blurred PTI-ETI dichotomy. Plant Cell 23:4-15.

Thomma, B.P., van Esse, H.P., Crous, P.W., and de Wit, P.J. (2005). Cladosporium fulvum (syn. Passalora fulva), a highly specialized plant pathogen as a model for functional studies on plant pathogenic Mycosphaerellaceae. Mol Plant Pathol 6:379-393.

Toruno, T.Y., Stergiopoulos, I., and Coaker, G. (2016). Plant-pathogen effectors: cellular probes 


\section{References}

interfering with plant defenses in spatial and temporal manners. Annu Rev Phytopathol 54:419441.

Toth, I.K., Bell, K.S., Holeva, M.C., and Birch, P.R. (2003). Soft rot erwiniae: from genes to genomes. Mol Plant Pathol 4:17-30.

Tripathi, J.N., Lorenzen, J., Bahar, O., Ronald, P., and Tripathi, L. (2014). Transgenic expression of the rice Xa21 pattern-recognition receptor in banana (Musa sp.) confers resistance to Xanthomonas campestris pv. musacearum. Plant Biotechnol J 12:663-673.

Trujillo, M., Ichimura, K., Casais, C., and Shirasu, K. (2008). Negative regulation of PAMP-triggered immunity by an E3 ubiquitin ligase triplet in Arabidopsis. Curr Biol 18:1396-1401.

Uhrig, R.G., Labandera, A.M., and Moorhead, G.B. (2013). Arabidopsis PPP family of serine/threonine protein phosphatases: many targets but few engines. Trends Plant Sci 18:505-513.

Underwood, W., Zhang, S., and He, S.Y. (2007). The Pseudomonas syringae type III effector tyrosine phosphatase HopAO1 suppresses innate immunity in Arabidopsis thaliana. Plant J 52:658-672.

van den Ackerveken, G.F., van Kan, J.A., and de Wit, P.J. (1992). Molecular analysis of the avirulence gene avr9 of the fungal tomato pathogen Cladosporium fulvum fully supports the gene-for-gene hypothesis. Plant J 2:359-366.

van den Burg, H.A., Harrison, S.J., Joosten, M.H., Vervoort, J., and de Wit, P.J. (2006). Cladosporium fulvum Avr4 protects fungal cell walls against hydrolysis by plant chitinases accumulating during infection. Mol Plant Microbe Interact 19:1420-1430.

van den Burg, H.A., Tsitsigiannis, D.I., Rowland, O., Lo, J., Rallapalli, G., Maclean, D., Takken, F.L., and Jones, J.D. (2008). The F-box protein ACRE189/ACIF1 regulates cell death and defense responses activated during pathogen recognition in tobacco and tomato. Plant Cell 20:697-719.

van den Burg, H.A., Westerink, N., Francoijs, K.J., Roth, R., Woestenenk, E., Boeren, S., de Wit, P.J., Joosten, M.H., and Vervoort, J. (2003). Natural disulfide bond-disrupted mutants of AVR4 of the tomato pathogen Cladosporium fulvum are sensitive to proteolysis, circumvent Cf-4-mediated resistance, but retain their chitin binding ability. J Biol Chem 278:27340-27346.

van der Burgh, A.M. (2018). SOBIR1-containing immune complexes at the plant cell surface: partners and signalling: Wageningen University \& Research $\mathrm{PhD}$ thesis.

van der Burgh, A.M., and Joosten, M.H.A.J. (2019). Plant immunity: Thinking outside and inside the box. Trends Plant Sci 24:587-601.

van der Burgh, A.M., Postma, J., Robatzek, S., and Joosten, M.H.A.J. (2019). Kinase activity of SOBIR1 and BAK1 is required for immune signalling. Mol Plant Pathol 20:410-422.

van der Hoorn, R.A., Laurent, F., Roth, R., and de Wit, P.J. (2000). Agroinfiltration is a versatile tool that facilitates comparative analyses of Avr9/Cf-9-induced and Avr4/Cf-4-induced necrosis. Mol Plant Microbe Interact 13:439-446.

van Esse, H.P., Bolton, M.D., Stergiopoulos, I., de Wit, P.J., and Thomma, B.P. (2007). The chitin-binding Cladosporium fulvum effector protein Avr4 is a virulence factor. Mol Plant Microbe Interact 20:1092-1101.

van Kan, J.A.L., van den Ackerveken, G.F.J.M., and de Wit, P.J.G.M. (1991). Cloning and characterization of cDNA of avirulence gene avr9 of the fungal pathogen Cladosporium fulvum, causal agent of tomato leaf mold. Mol Plant Microbe Interact 4:52-59.

Vert, G., Nemhauser, J.L., Geldner, N., Hong, F., and Chory, J. (2005). Molecular mechanisms of steroid hormone signaling in plants. Annu Rev Cell Dev Biol 21:177-201.

Vervoort, J., van den Hooven, H.W., Berg, A., Vossen, P., Vogelsang, R., Joosten, M.H., and de Wit, P.J. (1997). The race-specific elicitor AVR9 of the tomato pathogen Cladosporium fulvum: a cystine knot protein. Sequence-specific ${ }^{1} \mathrm{H}$ NMR assignments, secondary structure and global fold of the protein. FEBS Lett 404:153-158.

Voinnet, O., Rivas, S., Mestre, P., and Baulcombe, D. (2015). Retraction: An enhanced transient expression system in plants based on suppression of gene silencing by the $\mathrm{p} 19$ protein of tomato bushy stunt virus. Plant $\mathrm{J}$ 84:846.

Vossen, J.H., Abd-El-Haliem, A., Fradin, E.F., van den Berg, G.C., Ekengren, S.K., Meijer, H.J., Seifi, A., Bai, Y., ten Have, A., Munnik, T., et al. (2010). Identification of tomato phosphatidylinositol-specific phospholipase-C (PI-PLC) family members and the role of PLC4 and PLC6 in HR and disease resistance. Plant J 62:224-239.

Wan, W.-L., Zhang, L., Pruitt, R., Zaidem, M., Brugman, R., Ma, X., Krol, E., Perraki, A., Kilian, J., Grossmann, G., et al. (2018). Comparing Arabidopsis receptor kinase and receptor proteinmediated immune signaling reveals BIK1-dependent differences. New Phytol 221:2080-2095.

Wan, W.L., Frohlich, K., Pruitt, R.N., Nurnberger, T., and Zhang, L. (2019). Plant cell surface immune receptor complex signaling. Curr Opin Plant Biol 50:18-28.

Wang, G., Fiers, M., Ellendorff, U., Wang, Z., de Wit, P.J., Angenent, G.C., and Thomma, B.P. (2010a). 
The diverse roles of extracellular leucine-rich repeat-containing receptor-like proteins in plants. Crit Rev Plant Sci 29:285-299.

Wang, G., Long, Y., Thomma, B.P., de Wit, P.J., Angenent, G.C., and Fiers, M. (2010b). Functional analyses of the CLAVATA2-like proteins and their domains that contribute to CLAVATA2 specificity. Plant Physiol 152:320-331.

Wang, J., Grubb, L.E., Wang, J., Liang, X., Li, L., Gao, C., Ma, M., Feng, F., Li, M., Li, L., et al. (2018a). A regulatory module controlling homeostasis of a plant immune kinase. Mol Cell 69:493-504.

Wang, J., Li, H., Han, Z., Zhang, H., Wang, T., Lin, G., Chang, J., Yang, W., and Chai, J. (2015). Allosteric receptor activation by the plant peptide hormone phytosulfokine. Nature 525:265-268.

Wang, L., Albert, M., Einig, E., Furst, U., Krust, D., and Felix, G. (2016a). The pattern-recognition receptor CORE of Solanaceae detects bacterial cold-shock protein. Nat Plants 2:16185.

Wang, L., Einig, E., Almeida-Trapp, M., Albert, M., Fliegmann, J., Mithofer, A., Kalbacher, H., and Felix, G. (2018b). The systemin receptor SYR1 enhances resistance of tomato against herbivorous insects. Nat Plants 4:152-156.

Wang, R., Liu, M., Yuan, M., Oses-Prieto, J.A., Cai, X., Sun, Y., Burlingame, A.L., Wang, Z.Y., and Tang, W. (2016b). The brassinosteroid-activated BRI1 receptor kinase is switched off by dephosphorylation mediated by cytoplasm-localized PP2A B' subunits. Mol Plant 9:148-157.

Wang, X., and Chory, J. (2006). Brassinosteroids regulate dissociation of BKI1, a negative regulator of BRI1 signaling, from the plasma membrane. Science 313:1118-1122.

Wang, Y., Li, J., Hou, S., Wang, X., Li, Y., Ren, D., Chen, S., Tang, X., and Zhou, J.M. (2010c). A Pseudomonas syringae ADP-ribosyltransferase inhibits Arabidopsis mitogen-activated protein kinase kinases. Plant Cell 22:2033-2044.

Wang, Y., Xu, Y., Sun, Y., Wang, H., Qi, J., Wan, B., Ye, W., Lin, Y., Shao, Y., Dong, S., et al. (2018c). Leucine-rich repeat receptor-like gene screen reveals that Nicotiana RXEG1 regulates glycoside hydrolase 12 MAMP detection. Nat Commun 9:594.

Wang, Y.S., Pi, L.Y., Chen, X., Chakrabarty, P.K., Jiang, J., De Leon, A.L., Liu, G.Z., Li, L., Benny, U., Oard, J., et al. (2006). Rice XA21 binding protein 3 is a ubiquitin ligase required for full Xa21mediated disease resistance. Plant Cell 18:3635-3646.

Warren, R.F., Henk, A., Mowery, P., Holub, E., and Innes, R.W. (1998). A mutation within the leucine-rich repeat domain of the Arabidopsis disease resistance gene RPS5 partially suppresses multiple bacterial and downy mildew resistance genes. Plant Cell 10:1439-1452.

Westerink, N., Brandwagt, B.F., de Wit, P.J., and Joosten, M.H. (2004). Cladosporium fulvum circumvents the second functional resistance gene homologue at the Cf-4 locus (Hcr9-4E) by secretion of a stable avr4E isoform. Mol Microbiol 54:533-545.

Wierzba, M. (2013). A family of four LRR-RLKs modulate development and defense signaling in Arabidopsis thaliana through interaction with the co-receptor BAK1: University of Arizona PhD thesis.

Williams, R.W., Wilson, J.M., and Meyerowitz, E.M. (1997). A possible role for kinase-associated protein phosphatase in the Arabidopsis CLAVATA1 signaling pathway. Proc Natl Acad Sci USA 94:1046710472.

Win, J., Chaparro-Garcia, A., Belhaj, K., Saunders, D.G., Yoshida, K., Dong, S., Schornack, S., Zipfel, C., Robatzek, S., Hogenhout, S.A., et al. (2012). Effector biology of plant-associated organisms: concepts and perspectives. Cold Spring Harb Symp Quant Biol 77:235-247.

Wolf, S., van der Does, D., Ladwig, F., Sticht, C., Kolbeck, A., Schurholz, A.K., Augustin, S., Keinath, N., Rausch, T., Greiner, S., et al. (2014). A receptor-like protein mediates the response to pectin modification by activating brassinosteroid signaling. Proc Natl Acad Sci USA 111:15261-15266.

Wu, J., Liu, Z., Zhang, Z., Lv, Y., Yang, N., Zhang, G., Wu, M., Lv, S., Pan, L., Joosten, M.H., et al. (2016a). Transcriptional regulation of receptor-like protein genes by environmental stresses and hormones and their overexpression activities in Arabidopsis thaliana. J Exp Bot 67:3339-3351.

Wu, J., van der Burgh, A.M., Bi, G., Zhang, L., Alfano, J.R., Martin, G.B., and Joosten, M.H.A.J. (2018a). The bacterial effector AvrPto targets the regulatory coreceptor SOBIR1 and suppresses defense signaling mediated by the receptor-like protein Cf-4. Mol Plant Microbe Interact 31:75-85.

Wu, Q., Regan, M., Furukawa, H., and Jackson, D. (2018b). Role of heterotrimeric Ga proteins in maize development and enhancement of agronomic traits. PLoS Genet 14:e1007374.

Wu, S., Lu, D., Kabbage, M., Wei, H.L., Swingle, B., Records, A.R., Dickman, M., He, P., and Shan, L. (2011). Bacterial effector HopF2 suppresses Arabidopsis innate immunity at the plasma membrane. Mol Plant Microbe Interact 24:585-593.

Wu, W., Wu, Y., Gao, Y., Li, M., Yin, H., Lv, M., Zhao, J., Li, J., and He, K. (2015). Somatic embryogenesis receptor-like kinase 5 in the ecotype Landsberg erecta of Arabidopsis is a functional RD LRRRLK in regulating brassinosteroid signaling and cell death control. Front Plant Sci 6:852. 


\section{References}

Wu, Y., Xun, Q., Guo, Y., Zhang, J., Cheng, K., Shi, T., He, K., Hou, S., Gou, X., and Li, J. (2016b). Genome-wide expression pattern analyses of the Arabidopsis leucine-rich repeat receptor-like kinases. Mol Plant 9:289-300.

Wulff, B.B., Thomas, C.M., Parniske, M., and Jones, J.D. (2004). Genetic variation at the tomato Cf4/Cf-9 locus induced by EMS mutagenesis and intralocus recombination. Genetics 167:459-470.

Xiang, T., Zong, N., Zhang, J., Chen, J., Chen, M., and Zhou, J.M. (2011). BAK1 is not a target of the Pseudomonas syringae effector AvrPto. Mol Plant Microbe Interact 24:100-107.

Xiang, T., Zong, N., Zou, Y., Wu, Y., Zhang, J., Xing, W., Li, Y., Tang, X., Zhu, L., Chai, J., et al. (2008). Pseudomonas syringae effector AvrPto blocks innate immunity by targeting receptor kinases. Curr Biol 18:74-80.

Xiao, Y., Stegmann, M., Han, Z., DeFalco, T.A., Parys, K., Xu, L., Belkhadir, Y., Zipfel, C., and Chai, J. (2019). Mechanisms of RALF peptide perception by a heterotypic receptor complex. Nature 572:270-274.

Xin, X.F., and He, S.Y. (2013). Pseudomonas syringae pv. tomato DC3000: A model pathogen for probing disease susceptibility and hormone signaling in plants. Annu Rev Phytopathol 51:473498.

Xing, W., Zou, Y., Liu, Q., Liu, J., Luo, X., Huang, Q., Chen, S., Zhu, L., Bi, R., Hao, Q., et al. (2007). The structural basis for activation of plant immunity by bacterial effector protein AvrPto. Nature 449:243-247.

Xu, C., Liberatore, K.L., MacAlister, C.A., Huang, Z., Chu, Y.H., Jiang, K., Brooks, C., Ogawa-Ohnishi, M., Xiong, G., Pauly, M., et al. (2015). A cascade of arabinosyltransferases controls shoot meristem size in tomato. Nat Genet 47:784-792.

Xu, J., Wei, X., Yan, L., Liu, D., Ma, Y., Guo, Y., Peng, C., Zhou, H., Yang, C., Lou, Z., et al. (2013). Identification and functional analysis of phosphorylation residues of the Arabidopsis BOTRYTISINDUCED KINASE1. Protein Cell 4:771-781.

Xu, J., Xie, J., Yan, C., Zou, X., Ren, D., and Zhang, S. (2014). A chemical genetic approach demonstrates that MPK3/MPK6 activation and NADPH oxidase-mediated oxidative burst are two independent signaling events in plant immunity. Plant J 77:222-234.

$\mathrm{Xu}$, J., and Zhang, S. (2015). Mitogen-activated protein kinase cascades in signaling plant growth and development. Trends Plant Sci 20:56-64.

Yamaguchi, Y., Huffaker, A., Bryan, A.C., Tax, F.E., and Ryan, C.A. (2010). PEPR2 is a second receptor for the Pep1 and Pep2 peptides and contributes to defense responses in Arabidopsis. Plant Cell 22:508-522.

Yamaguchi, Y., Pearce, G., and Ryan, C.A. (2006). The cell surface leucine-rich repeat receptor for AtPep1, an endogenous peptide elicitor in Arabidopsis, is functional in transgenic tobacco cells. Proc Natl Acad Sci USA 103:10104-10109.

Yan, H., Zhao, Y., Shi, H., Li, J., Wang, Y., and Tang, D. (2018). BRASSINOSTEROID-SIGNALING KINASE1 phosphorylates MAPKKK5 to regulate immunity in Arabidopsis. Plant Physiol 176:29913002.

Yang, C.W., Gonzalez-Lamothe, R., Ewan, R.A., Rowland, O., Yoshioka, H., Shenton, M., Ye, H., O'Donnell, E., Jones, J.D., and Sadanandom, A. (2006). The E3 ubiquitin ligase activity of arabidopsis PLANT U-BOX17 and its functional tobacco homolog ACRE276 are required for cell death and defense. Plant Cell 18:1084-1098.

Yang, D., Shi, Z., Bao, Y., Yan, J., Yang, Z., Yu, H., Li, Y., Gou, M., Wang, S., Zou, B., et al. (2017). Calcium pumps and interacting BON1 protein modulate calcium signature, stomatal closure, and plant immunity. Plant Physiol 175:424-437.

Yang, M., and Sack, F.D. (1995). The too many mouths and four lips mutations affect stomatal production in Arabidopsis. Plant Cell 7:2227-2239.

Yasuda, S., Okada, K., and Saijo, Y. (2017). A look at plant immunity through the window of the multitasking coreceptor BAK1. Curr Opin Plant Biol 38:10-18.

Yeh, Y.H., Panzeri, D., Kadota, Y., Huang, Y.C., Huang, P.Y., Tao, C.N., Roux, M., Chien, H.C., Chin, T.C., Chu, P.W., et al. (2016). The Arabidopsis malectin-like/LRR-RLK IOS1 is critical for BAK1dependent and BAK1-independent pattern-triggered immunity. Plant Cell 28:1701-1721.

Yu, H., Yan, J., Du, X., and Hua, J. (2018). Overlapping and differential roles of plasma membrane calcium ATPases in Arabidopsis growth and environmental responses. J Exp Bot 69:2693-2703.

Yu, L.P., Simon, E.J., Trotochaud, A.E., and Clark, S.E. (2000). POLTERGEIST functions to regulate meristem development downstream of the CLAVATA loci. Development 127:1661-1670.

Yu, X., Feng, B., He, P., and Shan, L. (2017). From chaos to harmony: Responses and signaling upon microbial pattern recognition. Annu Rev Phytopathol 55:109-137.

Yuan, P., Jauregui, E., Du, L., Tanaka, K., and Poovaiah, B.W. (2017). Calcium signatures and signaling 
events orchestrate plant-microbe interactions. Curr Opin Plant Biol 38:173-183.

Zeng, L., Velasquez, A.C., Munkvold, K.R., Zhang, J., and Martin, G.B. (2012). A tomato LysM receptorlike kinase promotes immunity and its kinase activity is inhibited by AvrPtoB. Plant J 69:92-103.

Zhang, H., Lin, X., Han, Z., Qu, L.J., and Chai, J. (2016a). Crystal structure of PXY-TDIF complex reveals a conserved recognition mechanism among CLE peptide-receptor pairs. Cell Res 26:543-555.

Zhang, H., Lin, X., Han, Z., Wang, J., Qu, L.J., and Chai, J. (2016b). SERK family receptor-like kinases function as co-receptors with PXY for plant vascular development. Mol Plant 9:1406-1414.

Zhang, J., Li, W., Xiang, T., Liu, Z., Laluk, K., Ding, X., Zou, Y., Gao, M., Zhang, X., Chen, S., et al. (2010). Receptor-like cytoplasmic kinases integrate signaling from multiple plant immune receptors and are targeted by a Pseudomonas syringae effector. Cell Host Microbe 7:290-301.

Zhang, J., Shao, F., Li, Y., Cui, H., Chen, L., Li, H., Zou, Y., Long, C., Lan, L., Chai, J., et al. (2007). A Pseudomonas syringae effector inactivates MAPKs to suppress PAMP-induced immunity in plants. Cell Host Microbe 1:175-185.

Zhang, L., Kars, I., Essenstam, B., Liebrand, T.W.H., Wagemakers, L., Elberse, J., Tagkalaki, P., Tjoitang, D., van den Ackerveken, G.F.J.M., and van Kan, J.A.L. (2014). Fungal endopolygalacturonases are recognized as microbe-associated molecular patterns by the Arabidopsis receptor-like protein RESPONSIVENESS TO BOTRYTIS POLYGALACTURONASES1. Plant Physiol 164:352-364.

Zhang, M., Su, J., Zhang, Y., Xu, J., and Zhang, S. (2018). Conveying endogenous and exogenous signals: MAPK cascades in plant growth and defense. Curr Opin Plant Biol 45:1-10.

Zhang, S., and Klessig, D.F. (2001). MAPK cascades in plant defense signaling. Trends Plant Sci 6:520527.

Zhang, W., Fraiture, M., Kolb, D., Löffelhardt, B., Desaki, Y., Boutrot, F.F.G., Tör, M., Zipfel, C., Gust, A.A., and Brunner, F. (2013a). Arabidopsis receptor-like protein30 and receptor-like kinase suppressor of BIR1-1/EVERSHED mediate innate immunity to necrotrophic fungi. Plant Cell 25:4227-4241.

Zhang, Z., Fradin, E., de Jonge, R., van Esse, H.P., Smit, P., Liu, C.-M., and Thomma, B.P.H.J. (2013b). Optimized agroinfiltration and virus-induced gene silencing to study Ve1-mediated Verticillium resistance in tobacco. Mol Plant Microbe Interact 26:182-190.

Zhang, Z., Wu, Y., Gao, M., Zhang, J., Kong, Q., Liu, Y., Ba, H., Zhou, J., and Zhang, Y. (2012). Disruption of PAMP-induced MAP kinase cascade by a Pseudomonas syringae effector activates plant immunity mediated by the NB-LRR protein SUMM2. Cell Host Microbe 11:253-263.

Zhao, C., Nie, H., Shen, Q., Zhang, S., Lukowitz, W., and Tang, D. (2014). EDR1 physically interacts with MKK4/MKK5 and negatively regulates a MAP kinase cascade to modulate plant innate immunity. PLoS Genet 10:e1004389.

Zhou, J., Liu, D., Wang, P., Ma, X., Lin, W., Chen, S., Mishev, K., Lu, D., Kumar, R., Vanhoutte, I., et al. (2018). Regulation of Arabidopsis brassinosteroid receptor BRI1 endocytosis and degradation by plant U-box PUB12/PUB13-mediated ubiquitination. Proc Natl Acad Sci USA 115:E1906-E1915.

Zhou, J., Wu, S., Chen, X., Liu, C., Sheen, J., Shan, L., and He, P. (2014). The Pseudomonas syringae effector HopF2 suppresses Arabidopsis immunity by targeting BAK1. Plant J 77:235-245.

Zhou, Z., Zhao, Y., Bi, G., Liang, X., and Zhou, J.-M. (2019). Early signalling mechanisms underlying receptor kinase-mediated immunity in plants. Philos Trans R Soc Lond B Biol Sci 374:20180310.

Zhu, S., Li, Y., Vossen, J.H., Visser, R.G.F., and Jacobsen, E. (2012). Functional stacking of three resistance genes against Phytophthora infestans in potato. Transgenic Res 21:89-99.

Zhu, X., Caplan, J., Mamillapalli, P., Czymmek, K., and Dinesh-Kumar, S.P. (2010a). Function of endoplasmic reticulum calcium ATPase in innate immunity-mediated programmed cell death. EMBO J 29:1007-1018.

Zhu, Y., Wang, Y., Li, R., Song, X., Wang, Q., Huang, S., Jin, J.B., Liu, C.M., and Lin, J. (2010b). Analysis of interactions among the CLAVATA3 receptors reveals a direct interaction between CLAVATA2 and CORYNE in Arabidopsis. Plant J 61:223-233.

Zipfel, C. (2014). Plant pattern-recognition receptors. Trends Immunol 35:345-351.

Zipfel, C., Kunze, G., Chinchilla, D., Caniard, A., Jones, J.D.G., Boller, T., and Felix, G. (2006). Perception of the bacterial PAMP EF-Tu by the receptor EFR restricts Agrobacterium-mediated transformation. Cell 125:749-760.

Zipfel, C., Robatzek, S., Navarro, L., Oakeley, E.J., Jones, J.D., Felix, G., and Boller, T. (2004). Bacterial disease resistance in Arabidopsis through flagellin perception. Nature 428:764-767. 



\section{Summary}

Plants are continuously invaded by microbes, some of which are potential pathogens. Therefore, plants have evolved roughly two layers of defence to protect themselves against invaders. The first layer is mediated by cell surface receptors, which are either receptor-like kinases (RLKs) or receptor-like proteins (RLPs). RLKs contain a ligandbinding ectodomain, a transmembrane domain and an intracellular kinase domain. RLPs are structurally similar to RLKs concerning their ectodomain and transmembrane domain, but only contain a short cytoplasmic tail instead of a kinase domain. According to the motifs present in their ectodomains, cell surface receptors can be subdivided into different groups, with those containing leucine-rich repeat (LRR) motifs as the largest group. Activation of cell surface receptors upon recognition of extracellular immunogenic patterns (ExIPs) results in extracellularly-triggered immunity (ExTI) to arrest pathogen colonization. ExIPs can be any ExTI-triggering extracellular molecule, including microbe-associated molecular patterns (MAMPs) and extracellular effectors. Adapted pathogens suppress ExTI to colonize their host plants through the employment of effectors, of which the ones that are translocated into host cells can be recognized by intracellular receptors. These intracellular receptors are typically nucleotide binding leucine rich repeat proteins, and their activation results in intracellularly triggered immunity, which constitutes the second layer of plant defence.

So far, various LRR-containing RLKs and RLPs have been functionally characterized. For instance, the RLK FLAGELLIN-SENSING 2 (FLS2) recognizes the MAMP flg22, which is an immunogenic epitope of bacterial flagellin, to mediate defence against bacterial infection in higher plants. In addition, the RLK ELONGATION FACTOR-TU RECEPTOR (EFR) recognizes the MAMP elf18, which is an immunogenic epitope of bacterial elongation factor-Tu (EF-Tu), to mediate defence against bacterial infection in Brassicaceae. Furthermore, the tomato RLPs Cf-4 and Cf-9 confer resistance to strains of the biotrophic fungal pathogen Cladosporium fulvum secreting the effectors Avr4 and Avr9, respectively. RLPs constitutively interact with the regulatory RLK SUPPRESSOR OF BIR1-1 (SOBIR1) in a ligand-independent manner. In addition, both RLKs and RLPs form a ligand-dependent complex with the regulatory RLK BRI1ASSOCIATED RECEPTOR KINASE 1/SOMATIC EMBRYOGENESIS RECEPTOR KINASE 3 (BAK1/SERK3, further referred to as BAK1). The recruitment of BAK1 to RLKs and RLPs results in the activation of cell surface receptor complexes that subsequently activate downstream immune responses.

The work described in this thesis was aimed at gaining more insight into the molecular mechanisms of Cf-4/SOBIR1 signalling. In Chapter 1, the plant innate immune system is introduced. In addition, I provide an overview of the early signalling events occurring 
in RLK- and RLP-mediated immunity in this chapter, including examples of such events taking place in FLS2 and Cf-4 signalling, respectively. Furthermore, RLKs and RLPs involved in development are introduced, and an overview of the composition of RLK receptor complexes is provided.

Cf-4 constitutively interacts with SOBIR1 in a ligand-independent manner, and the Cf4/SOBIR1 complex recruits BAK1 upon Avr4 recognition. Establishment of the Cf4/SOBIR1/BAK1 complex is proposed to trigger phosphorylation of the intracellular kinase domains of SOBIR1 and BAK1, and subsequently trigger the development of a hypersensitive response (HR), which is a form of programmed cell death that restricts pathogen invasion. The components involved in controlling the Avr4-induced formation and subsequent activation of the Cf-4/SOBIR1/BAK1 complex remain unknown. Since the Arabidopsis thaliana (At, further referred to as Arabidopsis) RLKs BAK1INTERACTING RECEPTOR-LIKE KINASE 2 (BIR2) and BIR3 (further referred to as BIR2/3), were previously reported to negatively regulate FLS2 signalling through controlling the establishment of the FLS2/BAK1 complex, we set out to study whether BIR2/3 also play a role in Cf-4 signalling (Chapter 2). Transient overexpression of AtSOBIR1 in Nicotiana benthamiana triggers constitutive immunity that reflects Avr4activated Cf-4 signalling, and provides a tool to study the signalling events occurring downstream of the $\mathrm{Cf}-4 / \mathrm{SOBIR} 1$ complex. We show that BIR2/3 interact with AtSOBIR 1 and suppress AtSOBIR1-triggered constitutive immunity. In addition, BIR2/3 likely target endogenous $\mathrm{NbSOBIR} 1$, thereby inhibiting the association of endogenous NbSOBIR1 with Cf-4 and suppressing the Cf-4/Avr4-triggered HR. We also show that AtSOBIR1, but not the kinase-inactive mutant AtSOBIR1 ${ }^{\mathrm{D} 489 \mathrm{~N}}$, phosphorylates BIR2/3. Furthermore, the interaction with BIR2/3 is more apparent for AtSOBIR1 ${ }^{\mathrm{D} 489 \mathrm{~N}}$ than for AtSOBIR1. These observations suggest that BIR2/3 interact with SOBIR1 in the resting state, while perception of Avr4 triggers auto-phosphorylation of SOBIR1 that subsequently phosphorylates BIR2/3, leading to their dissociation from SOBIR1.

As SOBIR1 is required for RLP-mediated defence against infection by a wide range of pathogens, we hypothesized that SOBIR1 might be targeted by intracellular effectors to suppress Cf-4/SOBIR1-mediated signalling. In Chapter 3, it is shown that AvrPto, an effector of the bacterial pathogen Pseudomonas syringae pv. tomato (Pst) DC3000, interacts with SOBIR1 from Arabidopsis, tomato and $N$. benthamiana. This interaction is independent of SOBIR1 kinase activity. We show that overexpression of AvrPto suppresses AtSOBIR1-triggered constitutive immunity, as well as the Cf-4/Avr4triggered HR without affecting Cf-4/SOBIR1/BAK1 complex formation. Collectively, these results suggest that AvrPto targets the regulatory RLK SOBIR1 to compromise plant defence. 
Studies on chimeric cell surface receptor proteins revealed that the ectodomain of a chimera retains the ability to perceive the ligand, whereas the transmembrane and cytoplasmic domains determine the signalling output. MAMP-triggered immunity generally results in a relatively weak immune response when compared with effectortriggered immunity. In Chapter 4, we aimed to obtain a strong MAMP-dependent immune response in $N$. tabacum by exploiting the EFR-mediated recognition of the MAMP elf18 and the ability of Cf-9 to trigger a strong HR. To this end, the chimeric receptor protein EFR-Cf-9 was generated by fusing the ectodomain of EFR to the transmembrane and cytoplasmic domains of Cf-9. We show that transiently overexpressed EFR-Cf-9 recognizes elf18 to trigger a strong HR in $N$. tabacum. In addition, EFR-Cf-9 interacts with SOBIR1, and requires both SOBIR1 and BAK1 for its functionality. Moreover, transgenic $N$. tabacum plants expressing EFR-Cf-9 do not display altered basal defence, but recognize elf18 to trigger an HR. These transgenic plants are resistant to the biotrophic bacterial pathogens Pst DC3000 and P. amygdali pv. tabaci (Pta) 11528, through recognition of pathogen-derived EF-Tu. Notably, these transgenic plants even mount an HR upon inoculation with a high dose of Pta 11528. Taken together, these results indicate that the chimeric receptor EFR-Cf-9 recognizes elf18 to confer strong SOBIR1- and BAK1-dependent resistance to biotrophic bacterial pathogens.

Arabidopsis CLAVATA2 (CLV2) is an RLP that associates with the pseudokinase CORYNE (CRN) to participate in regulation of the activity of the shoot apical meristem, mediated by the endogenously secreted peptide CLV3. The loss-of-function mutants $c / v 2, c r n$ and $c / v 3$ display various developmental phenotypes, including an increased number of rosette leaves when compared to wild-type plants. Previously, an interaction between tomato SOBIR1 and CLV2 was reported. However, the significance of this interaction remains unknown. In Chapter 5, we show that Arabidopsis CLV2 not only interacts with SOBIR1, but also with BAK1. Hence, we set out to investigate the significance of these interactions, in relation to SOBIR1- and/or BAK1-dependent immune responses mediated by RLPs and FLS2, and in relation to CLV2-dependent CLV3 signalling. We show that the CLV2/CRN complex positively regulates immune responses mediated by RLPs and FLS2, probably through facilitating the accumulation of the SOBIR1 and BAK1 proteins. In addition, SOBIR1 participates in the CLV3mediated regulation of the rosette leaf number, probably through facilitating CLV2 accumulation. However, whether BAK1 plays a role in this process remains unknown. In this chapter, we also aimed to identify downstream components required for CLV3 signalling. We show that the Arabidopsis G-protein $\beta$ subunit AGB1 and the G-protein Y subunits $A G G 1$ and $A G G 2$, which are required for FLS2 signalling and CLV3 
signalling in the shoot, are also required for suppression of root elongation upon exogenous application of a synthetic CLV3 peptide. However, other $\mathrm{G}$ protein subunits that are required for FLS2 signalling are not required for this process. Furthermore, we show that many additional components that are required for FLS2 signalling are not required for CLV3 signalling in the root. We conclude that the requirement of downstream components for signalling triggered by flg22 on the one hand, and CLV3 on the other hand, is different.

In Chapter 6, the main results of the work described in this thesis are discussed and placed in a broader perspective. I provide an overview of our current understanding of the regulation of LRR-type cell surface receptor complexes involved in defence and development, with a focus on the regulators controlling the formation, activation and abundance of cell surface receptor complexes. 


\section{Acknowledgements}

Time flies, my PhD journey finally comes to its last period. Looking back at the past five years, I would like to take the opportunity to thank all the people who were involved in this amazing experience.

My dear co-promoter Matthieu. Thanks for offering me the opportunity to study abroad. This thesis would not have been possible without your support and effort. I am fully convinced that finishing my PhD journey under your supervision will be my lifelong memorable experience. Thank you, and I wish you all the best with your career and with your life in the future.

My thanks also go to my promoter, Bart. I really appreciate your feedback on my writing. Thank you, and I wish you all the best in the future.

All the current and former members of the SOL group, thanks for creating a warming environment. One of the best experiences during my $\mathrm{PhD}$ journey was to have the opportunity to work with all of you. Aranka, I cannot imagine how hard it would have been for my PhD journey without your help. You are a wonderful colleague who was always helpful in and outside the lab, and you are a nice friend who made my life in Wageningen easier. Laurens (big flexible body, BFB), you are the one I always want to work with. Thanks for teaching me experimental and dance skills. I also want to thank Daniela, Guozhi and Yu for helping me to get started. Wen and Sergio, thanks for the discussions, and I wish you both lots of success in your PhD research! Special thanks go to my students Michelle, Raquel, Ziqi and Xiaoyu. I really enjoyed working with all of you, and I wish you all the best in your career!

Special thanks to all PhDs at Phytopathology for all the interesting discussions. It was my pleasure to work with you, and I wish you all the best in your PhD research. I also want to thank all staff members at Phytopathology for providing supports during my PhD journey.

My thanks also go to Unifarm personnel. Bert, Henk, Gerrit and Taede, thanks for providing and taking care of the plants.

Many thanks to Guozhi, Wen, Yin, Jinling, Hui, Xiaoqian, Si, Yaohua, Yu, Yan, Shuqing and Weizhen for the talks and dinners together. I also want to thank my Chinese friends in the Netherlands. Kaile Sun, Yiqian Fu, Xiao Lin, Xiaoxue Sun, Xuexue Sun, Mengjing Sun \& Wei Du, Huayi Li, Yajun Wang, Xu Cheng, Huchen Li, Defeng Shen, Tian Zeng, 
Jieyu Liu, Fengjiao Bu, Wenkun Zhou, Bing Bai, Shuang Song, Rufang Wang, Qi Zheng, Minggang Wang, Chen Zhang, Zhibin Liu and Feng Zhu. I really appreciate the moments we had together and wish you all great success in your careers.

All of this would not be possible without my family. I would like to express my great gratitude to my parents for their endless love and unconditional support. Last but not least, special thanks to my sisters and their families. Thanks for your support and contribution to our family, and I wish that all of you have a happy and healthy life!

Jinbin Wu (吴锦斌)

Wageningen

December 13, 2019 


\section{About the author}

Jinbin Wu (吴锦斌) was born on October $30^{\text {th }}$, 1988 in Longyan City, Fujian Province, China. In 2007, he started his Bachelor study majoring in Life Sciences at Northwest University in Xi'an City, Shaanxi Province, China. After graduation in July 2011, he started his Master study majoring in Botany at Shaanxi Normal University in Xi'an. He performed his MSc thesis on functional analysis of Arabidopsis RLPs and CLEs involved in regulation of vascular development under the supervision of Prof. Guodong Wang. In 2014, he obtained a PhD fellowship from the China Scholarship Council (CSC) to join the Laboratory of Phytopathology at Wageningen University, the Netherlands. Under the supervision of Dr. Matthieu Joosten, Jinbin conducted his PhD research resulting in the publication of this thesis, entitled "Regulation and activation of SOBIR1containing receptor complexes involved in plant immune signalling". 


\section{List of publications}

Wu J\#, Reca I-B\#, Spinelli F, Lironi D, De Lorenzo G, Poltronieri P, Cervone F, Joosten MHAJ, Ferrari S, Brutus A (2019) An EFR-Cf-9 chimera confers enhanced resistance to bacterial pathogens by SOBIR1- and BAK1-dependent recognition of elf18. Mol. Plant Pathol. 20(6): 751-764.

Wu J, van der Burgh AM, Bi G, Zhang L, Alfano JR, Martin GB, Joosten MHAJ (2018) The bacterial effector AvrPto targets the regulatory co-receptor SOBIR1 and suppresses defence signalling mediated by the receptor-like protein Cf-4. Mol. Plant Microbe Interact. 31(1): 75-85.

Wu J\#, Liu Z\#, Zhang Z\#, Lv Y, Yang N, Zhang G, Wu M, Lv S, Pan L, Joosten MHAJ, Wang G (2016) Transcriptional regulation of receptor-like protein genes by environmental stresses and hormones and their overexpression activities in Arabidopsis thaliana. J. Exp. Bot. 67(11): 3339-3351.

Qiang Y\#, Wu J\#, Han H, Wang G (2013) CLE peptides in vascular development. J. Integr. Plant Biol. 55(4): 389-394.

Zhang L, Shi X, Zhang Y, Wang, Yang J, Ishida T, Jiang W, Han X, Kang J, Wang X, Pan L, Lv S, Cao B, Zhang Y, Wu J, Han H, Hu Z, Cui L, Sawa S, He J, Wang G (2019) CLE9 peptide-induced stomatal closure is mediated by abscisic acid, hydrogen peroxide, and nitric oxide in Arabidopsis thaliana. Plant Cell Environ. 42(3): 1033-1044. Lv S, Zhang Y, Li C, Liu Z, Yang N, Pan L, Wu J, Wang J, Yang J, Lv Y, Zhang Y, Jiang W, She X, Wang G (2018) Strigolactone-triggered stomatal closure requires hydrogen peroxide synthesis and nitric oxide production in an abscisic acid-independent manner. New Phytol. 217(1): 290-304.

Pan L, Lv S, Yang N, Lv Y, Liu Z, Wu J, Wang G (2017) The multifunction of CLAVATA2 in plant development and immunity. Front. Plant Sci. 7: 1573.

Lv Y, Yang N, Wu J, Liu Z, Pan L, Lv S, Wang G (2016) New insights into receptorlike protein functions in Arabidopsis. Plant Signal. Behav. 11(7): e1197469.

\#equal contribution 


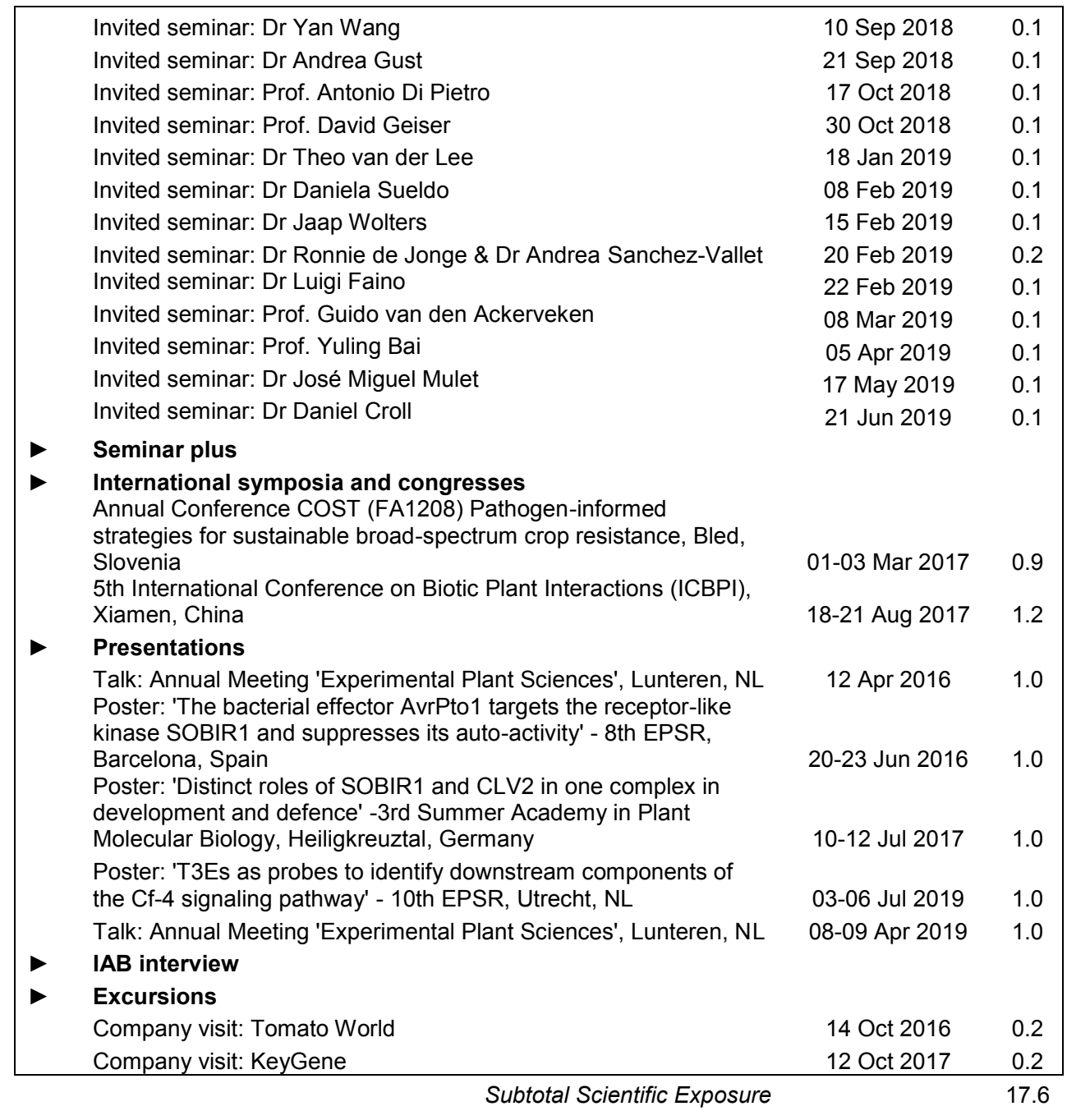

\begin{tabular}{|lcr|}
\hline 3) In-Depth Studies & $\underline{\text { date }}$ & $\underline{c p}$ \\
$\quad \begin{array}{l}\text { Advanced scientific courses \& workshops } \\
\text { 3rd Summer Academy in Plant Molecular Biology, Heiligkreuztal, } \\
\text { Germany }\end{array}$ & $10-12$ Jul 2017 & 0.9 \\
$\begin{array}{l}\text { 10th Utrecht PhD Summer School 'Environmental Signaling in } \\
\text { Plants', Utrecht, NL }\end{array}$ & $26-28$ Aug 2019 & 0.9 \\
$\quad \begin{array}{l}\text { Journal club } \\
\text { Member of a literature discussion group at Phytopathology }\end{array}$ & $2014-2019$ & 3.0 \\
$\quad$ Individual research training & & \\
\hline
\end{tabular}




\begin{tabular}{|lcc|}
\hline 4) Personal Development & $\underline{\text { date }}$ & $\underline{c p}$ \\
$\quad$ General skill training courses & Jan-Feb 2015 & 0.0 \\
English for IELTS & Mar-Jun 2015 & 0.0 \\
Practical English Plus & 22 Sep 2015 & 0.2 \\
EPS introduction course & 08 Apr 2016 & 0.3 \\
WGS PhD Workshop Carousel & 07 Apr 2017 & 0.3 \\
WGS PhD Workshop Carousel & 06 Feb 2018 & 0.3 \\
Career day & 07 May 2019 & 0.2 \\
Infographics and Iconography & Apr-Jun 2019 & 1.8 \\
Scientific writing & & \\
Organisation of meetings, PhD courses or outreach activities & & \\
Membership of EPS PhD Council & & 3.1 \\
\hline
\end{tabular}

Subtotal Personal Development

Herewith the Graduate School declares that the PhD candidate has complied with the educational requirements set by the Educational Committee of EPS with a minimum total of 30 ECTS credits.

${ }^{*} A$ credit represents a normative study load of 28 hours of study. 
This work was carried out in the Laboratory of Phytopathology, Wageningen University \& Research, the Netherlands. Jinbin Wu was financially sponsored by a PhD fellowship from the China Scholarship Council (CSC). Financial support from Wageningen University for printing this thesis is gratefully acknowledged.

Cover and layout design: Jinbin Wu

Printed by: ProefschriftMaken 


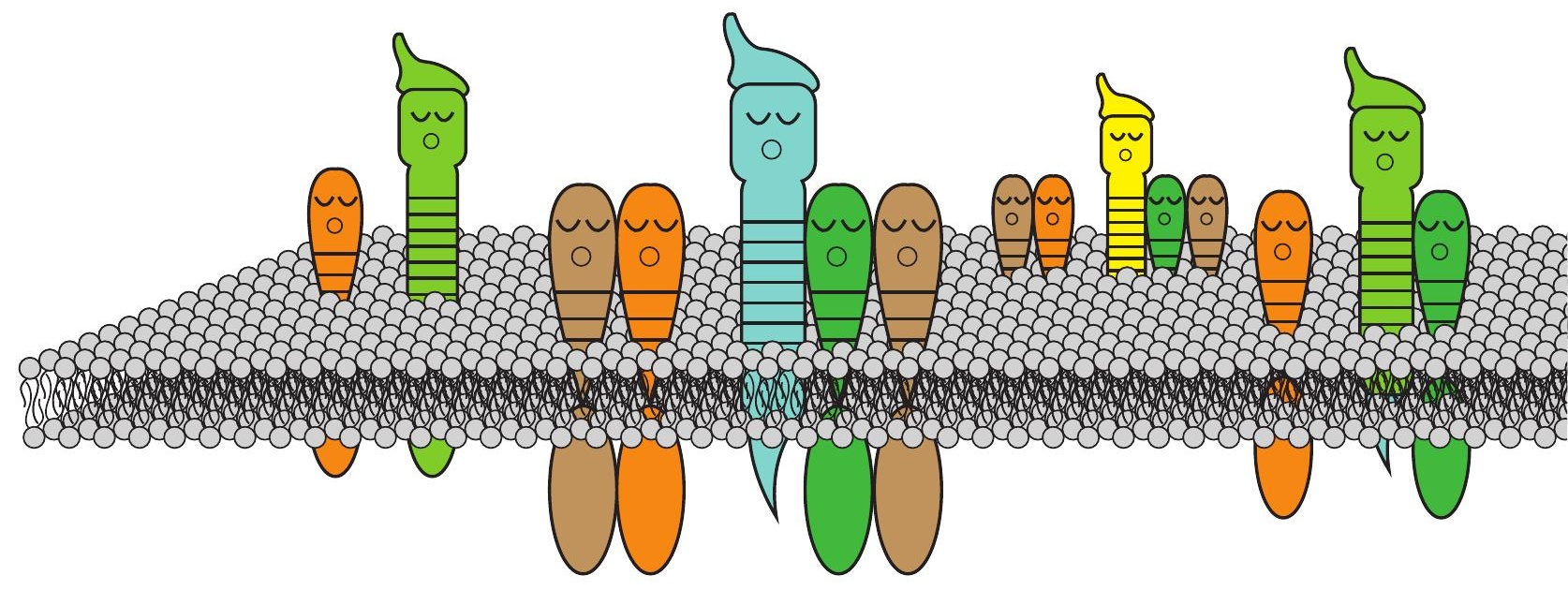

